Digitized by the Internet Archive in 2007 with funding from Microsoft Corporation 

Ist Edition, May, 1917.

R'eprinted August, 1917.

2nd Edition, February, 1918. 


\section{THE TREATMENT OF INFECTED WOUNDS}

BY

A. CARREL AND G. DEHELLY

TRANSLATION BY

HERBERT CHILD

CAPT. R.A.M.C. (TY.), FORMERLY SURGEON, FRENCH RED CROSS

WITH INTRODUCTION BY

SIR ANTHONY A. BOWLBY

K.C.M.G., K.C.V.O., F.R.C.S., SURGEON-GENERAL, ARMY MEDICAL SERVICE ADVISING CONSULTING SURGEON TO THE BRITISH ARMIES IN FRANCE

\section{SECOND EDITION}

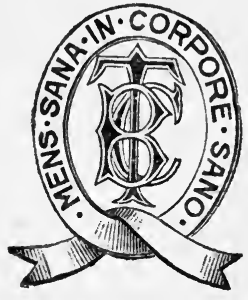

- LONDON

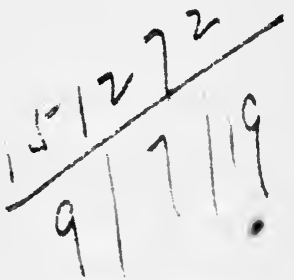

BAILLIERE, TINDALL AND COX

8, HENRIETTA STREET, COVENT GARDEN

I 9 I 8 
PRINTED IN GREAT BRITAIN. 


\section{INTRODUCTION}

I HAVE been asked to write an Introduction to the English edition of the work by Dr. A. Carrel and Dr. G. Dehelly, and I am glad to take the opportunity of expressing the appreciation of British surgeons at the Front of the value of what is known to us as "Carrel's Method."

Whenever it has been thoroughly carried out it has accomplished all that is claimed for it by its author, and it has been of inestimable benefit to thousands of patients. It has also renewed faith in antiseptic methods, in spite of the attacks on their utility which characterised the early stages of the war, and has done the greatest good by setting a high standard of thorough excision and surgical cleanliness. The whole practice of war surgery has been greatly improved by Dr. Carrel's confidence that antiseptic treatment can sterilise a septic wound, and that it does do so if sufficient care and skill are bestowed upon it; and the lesson he has taught was very necessary.

The book itself will be found to convey in the clearest manner the knowledge of those details which have been so carefully elaborated by the patient work of two years' experience, but it is only by scrupulous attention to every detail that the best results will be obtained. 
I would also suggest that, if "Carrel's Method" is to be fairly judged, no change whatever should be made either in the Dakin's solution itself, or in the use of the tubes for instilling it. The tendency has often been so to modify these details, in the belief that they were thereby "improved," that the author himself would be the first to disclaim the improved methods which are yet called by his name. The only modification that seems justifiable is the use of the syringe when instillation by gravitation cannot be carried out, as in trains, ships, and many units at the Front. It will be time enough to introduce other modifications after a prolonged trial of the methods advised in this publication.

The utility of Carrel's method is not confined to recent wounds, and, in the following pages, those surgeons who are treating the wounded in Great Britain will find all the necessary information for the treatment of both healthy and suppurating wounds.

The Army Medical Department has already arranged that, in those cases where it is employed, this treatment can be continuously carried out not only in the Front and Base Hospitals, but also in Ambulance Trains, Hospital Ships, and Hospitals in Great Britain.

To the workers in each of these areas of surgery this book will prove of the utmost practical value, and I feel certain it will be of the greatest value of ail to the patients themselves.

\section{ANTHONY A. BOWLBY.}

General Headquarters,

B.E.F., FRANCE, February, 1918. 


\section{PREFACE TO THE SECOND EDITION}

IN this New Edition will be found a description of the technical improvements which have been effected during the last few months. We have also thought it desirable to demonstrate the fresh results which have been obtained in the treatment of old wounds, old fractures, and other surgical affections.

CoMpIÈgne, I9I 7. 



\section{PREFACE TO THE FIRST EDITION}

THE researches which are dealt with in this book were made in the laboratories established at Compiegne by the Rockefeller Foundation, and at the Temporary Hospital, No. 2 I, under the Service du Santé militaire.

The chemical laboratory was directed by Dr. Henry D. Dakin, ${ }^{1}$ who there made the experiments upon which the sterilisation of wounds is founded. In the biological part of his experiments, Dr. Dakin was assisted by M. Daufresne and Mme Carrel. Chemical research was carried on in the same laboratory by M. Daufresne. Le médecin-major Vincent, of the colonial forces, directed the bacteriological laboratory. The mathematical and physical portion of the researches was done by $\mathbf{M}$. Lecomte du Nouy and M. Jaubert de Beaujeu. The physiological and surgical experiments were made with the help of Miss Lilly. Photography by MM. Pierre Magnier and Baillergeau.

The wounded were treated successively by M. Dumas, and by le médecin-major Bernoud of the colonial forces, then by MM. Woimant, Audiganne, and Guillot. MM.

${ }^{1}$ Dr. H. D. Dakin, Director of the Herter Laboratory, New York, was one of the candidates selected by the Council of the Royal Society to be recommended for election into the Society. (B. M. J., March I3, 1917 . Translator's note.) 
Guillot and Woimant dealt with the surgical experiments. Clinical investigations into the cicatrisation of wounds were made by Mlle Hartmann and by Mme Carrel. M. Jaubert de Beaujeu was in charge of the radiological laboratory.

The administration of the hospital and the laboratories was in the hands of les officiers d'administration Biérer and Bois, successively.

The nursing was done by the infirmières of l'Ecole de la Source, superintended by Mme Carrel, by Mlle Weilenmann and Mlle Junod, and by the American nurses of the "Post Unit."

The military administration of the hospital was directed by médecin-major Bernoud, of the colonial forces.

The hospital was controlled by M. le Sous-Secrétaire d'Etat du Service de Santé, and all the details of its organisation and administration were in charge of $\mathrm{M}$. de Piessac, of the Sous-Secrétariat d'Etat.

\section{A. CARREL.}

Compiègne,

September 1, 1916. 


\section{CONTENTS}

CHAP.

Introduction by Sir Anthony A. Bowlby . . . V

Preface to the Second Edition . . . . . vii

Preface to the First Edition . . . . . . ix

Introduction ..$\quad$. . . . . . . I

I. The Principles of the Technique . . . . . 14

II. The Technique of the Manufacture of Dakin's Solution 93

III. The Technique of the Sterilisation of Wounds-

Mechanical, Chemical, and Surgical Cleansing • Io9

IV. The Technique of the Sterilisation of Wounds-

Chemical Sterilisation . . . . . . I35

V. The Clinical and Bacteriological Examination of

Wounds . . . . . . : . 173

VI. The Closure of Wounds . . . . . 205

VII. The Results . . . . . . . . . 216

Appendix.-Chloramine Paste . . . . 258

Index . . . . . . . . 259 



\section{THE TREATMENT \\ OF INFECTED WOUNDS}

\section{INTRODUCTION}

I. IT is well known that nearly all the wounds resulting from explosions of shells, torpedoes, and bombs are septic; and that the methods employed up to the present in the treatment of these wounds are generally impotent to check the progress of the infection. To be convinced of this, one has only to be present at the arrival at a base hospital of a convoy of wounded, who have been operated on in the dressing-stations or the hospitals near the front. Then one grasps the danger of those paradoxes upheld by surgeons who still deny the universality of infection.

That the septic character of wounds is disastrous is also well known. During the early hours, or the first few days, the wound is exposed to the danger of gas-producing infection. Later are developed the various infections, which, either in the seat of fracture, in joints laid open, or in extensive lacerations of soft parts, sometimes give rise to lesions leading to amputation or to death. At the hospital of the Maison Blanche, M. Tuffier, as a result of the examination of a large number of cases of amputation, found that about 70 per cent. of the operations 
were needed because of the presence of infection, and were not due to the extent of the anatomical lesions.

Even when the patient has had the good fortune to be operated on close to the scene of action by a competent surgeon, and has escaped the serious infections of the early stages, suppuration still occurs, and continues indefinitely. Sometimes it becomes a danger to life or limb, and almost always brings about adhesions between muscles, aponeuroses, tendons, nerves and vessels. After healing, the patient has scars of large area, often painful, which prevent the limb from resuming its normal functions. Tendons remain gripped in fibrous fetters. Nerve extremities which have been bathed for weeks in pus, sclerose. Deep in infected bones, osteo-myelitis springs up. For months, maybe years, the limb still suppurates. Joints ankylose, muscles atrophy, and the wounded man becomes unfit, not only for being a soldier, but for work of any kind.

The suppression of wound infection would protect a large number of men from incapacity or death, and would bring about the rapid restoration to health of the greater number of those whose anatomical lesions are compatible with life. Such progress would result in great saving in money and men.

It would seem, however, that hitherto practically no really systematic research has been carried out with the object of discovering the procedure needful to bring about this improvement in treatment of wounded. As a matter of fact, attempts have been made by isolated individuals and often with extemporised equipment. Experimenters have attempted, working alone, researches which needed the co-ordinated efforts of chemists, pathologists, 
bacteriologists, trained in scientific technique. Proceedings of learned societies are laden with reports, based for the greater part on experiments and observations, incomplete, vitiated by faulty methods. No results of value were obtained. Despite the academic toil of many surgeons, wounds suppurate to-day as freely as ever.

It is known, however, that, under certain conditions, infected wounds can be rendered sterile. Lister, undoubtedly, by the aid of carbolic acid, succeeded in disinfecting compound fractures, at a time when such an injury was of the gravest import. Nevertheless, modern surgeons disregard these facts. Not only have they despised the road opened up by Lister, but they even question the possibility of applying the principle of antiseptics to war-wounds.

The throwing-over of Lister's ideas came about, not so much from the inadequacy of his method, as from the carelessness with which it was applied. In clinical researches, the basic principles of scientific investigation were forgotten. Methods utilising measurements were rarely employed. In the wounds investigated, it was never sought to estimate exactly the relations which exist between the number of microbes present, their nature, and the rapidity of cicatrisation. Any substance which possessed the property of destroying microbes in vitro, was looked upon as an antiseptic, and used in the treatment of wounds, every man to his taste. Substances which coagulated proteids, which lost their bactericidal power in the presence of serum, or which were actually harmful to the tissues, were all used. What degree of concentration of a bactericidal substance was to 


\section{TREATMENT OF INFECTED WOUNDS}

be used at the surface of a wound, how this degree of concentration was to be maintained-such details were never sought. The period during which this substance should remain on the surface of the wound, at a given concentration, was never determined. No careful study was made of the quantitative modifications, produced by the antiseptic agent, of the microbial flora, modifications which can only be revealed by daily bacteriological examination. The action of antiseptics on tissue repair was ignored, although it was important to learn how much the substances employed would impede the progress of cicatrisation. In a word, in the therapeutics of septic wounds, we may attribute the stagnation we have experienced to the lack of precision in clinical research.

However, Lister's method was held responsible for technical inadequacies, and surgeons raised to the position of a dogma the teaching that antiseptics had no real efficacy. In a memorandum on the treatment of wounds in war, ${ }^{1}$ MM. Burghard, Leishman, Moynihan and Wright, wrote in April, 1915, that "the treatment of suppurating wounds by means of antiseptics is illusory, and that belief in its efficacy is founded upon false reasoning." The principal adversary of antisepsis was Sir Almroth Wright. He believed that Lister's method was not applicable to war-wounds, and that the microbes, being carried by projectiles and fragments of clothing deep into the tissues, were beyond the reach of antiseptics. Chemical sterilisation of a wound seemed to him impossible of realisation. "In fact," he wrote in

1 Burghard, Leishman, Moynihan and Wright, Office international d'hygiene publique, 1915, vol. vii. p. 946. 
1915, "if it were ever to come about that an antiseptic sterilised heavily infected wounds, that would be a matter to announce in all the evening and morning papers." 1

Although Sir Almroth Wright's doctrine was founded, not on observations and experiments made upon wounds under actual war conditions, but upon ingenious theories and experiments in vitro, it was accepted by the majority of surgeons. One of them even affirms that asepsis ought to take the place of antisepsis, and that antiseptics not only fail to sterilise wounds, but that they actually favour the development of microbes! ${ }^{2}$ Therefore wounds came to be treated with saline solutions, or the hypertonic solutions of Wright. The fundamental observations of Lister were forgotten completely. Nevertheless, theories remained impotent and infection flourished.

II. However, setting aside theory and confining oneself closely to fact, the problem of wound sterilisation seems very simple. It is to be remembered that the surgical infection, at the outset, is always local. In war-wounds, it is carried by projectiles, and especially by fragments of clothing, impregnated with micro-organisms. Before crossing the boundaries of the wound, these flourish on the surface of the tissues. Therefore, during a period more or less long, the infection is under control, since the microbes are, so to speak, within reach of the hand. The question then is simply, how to destroy them without harming the tissues?

1 Sir A. Wright. An Address on Wound Infections. British Medical Fournal, April 24, 1915, p. 721.

'Pierre Delbet, Bulletin et Memoires de la Societe de Chirurgie, Janvier, I916. 
As a difference of resistance exists between, on the one hand, the tissues provided with a circulation, and, on the other hand, microbes, isolated anatomical elements and necrosed tissues, it was useless to seek a substance which would exercise an elective action on microorganisms. Better seek to discover, for a given antiseptic, that degree of concentration of the solution, and that length of time during which it must be applied, which, fatal to microbes, will not produce obvious damage to the tissues.

When, at the end of December, 1914, Henry D. Dakin and one of the authors of this book sought to discover the best means of treating wound-infections, they adopted, for the reasons just stated, the method of chemio-therapy. Besides, it seemed probable that the infection of war-inflicted wounds would be unsuitable for treatment by vaccines or serums. As the inoculation of the tissues by projectiles or fragments of clothing is massive, and as the germs protected by necrosed tissues or blood-clots multiply beyond the reach of lymph-flow, it is extremely unlikely that such therapeusis could be effective. Besides, the bacterial flora of war-wounds is extremely varied. As the large numbers of wounded and the conditions of the dressing-stations render impossible identification of the microbes which infect the wounds, it would be necessary to use vaccines or serums against dozens of types of germs, aërobic and anaërobic. Failure of such an attempt is foredoomed.

On the other hand, the problem would appear to be readily solved by using a substance unirritating to the tissues, and of a sufficient bactericidal power to kill all the microbes present in a wound, be their nature what it may. 
It was in this direction that the researches tended. Dakin studied the action on tissues and micro-organisms of' a considerable number of antiseptics, old and new. More than two hundred substances were examined in this manner by him, and he was led, for various reasons, to make chloramines, ${ }^{1}$ and, by a special process, hypochlorite of soda. ${ }^{2}$ Thanks to his excellent researches, we quickly had placed at our disposal substances endowed with feeble irritating qualities, with a toxicity for the organism almost nil, but of considerable bactericidal power. We then studied under what conditions these substances could sterilise a wound. These researches demonstrated that the microbes disappeared, if the antiseptic remained in contact with the surface of the wound at a certain degree of concentration during a prolonged period. Bacteriological examination showed that infected wounds, treated according to these principles, became sterile. Thus, quite simply, was realised what Sir Almroth Wright and modern surgeons consider to be impossible.

III. The method was applied in the first place to old wounds, afterwards to recent ones. Sterilisation was attained in both cases, but the earlier treatment gave the more rapid results. It has long been admitted that preventive treatment of a malady costs less in money and toil than curative treatment. However, infection can be checked even after suppuration has become established. In a word, all infected wounds were brought more or less under control by chemiotherapy.

1 Dakin, Cohen, Daufresne and Kenyon, Proceedings of the Royal Society, 1916, vol. 89, p. 232.

2 Dakin, Presse Médicale, Sept. 30, 1915. 


\section{TREATMENT OF INFECTED WOUNDS}

From May, 1915, it became evident that wounds treated after a certain method by the aid of hypochlorite or the chloramines of Dakin were sterilised, without any harm resulting to the tissues or the patient. From that date, it has been possible to prevent, in the greater number of cases, infection of wounds, and to abolish, almost entirely, suppuration in hospitals. At this time the method was practised in some hospitals of the first line by le médecin principal Uffoltz, Directeur du Service de santé d'un corps d'armée. To him and to his colleagues is due the merit of demonstrating that infection of wounds treated under the ordinary conditions of a field hospital can be almost entirely done away with.

During the months of July and September, I915, Dakin ${ }^{1}$ published an account of the substances which gave these results, and the mode of preparation. In October of the same year, M. Pozzi demonstrated in our name at the Académie de Médecine the principles which are fundamental to the chemiotherapy of wounds. ${ }^{2}$ These principles were later set forth in a more complete manner in 1916, in the Archives de Médecine et de Pharmacie Militaires. ${ }^{3}$ On several occasions at the Académie de Médecine, and at the Société de Chirurgie, M. Pozzi, ${ }^{4}$ spoke at length upon the technique and upon the results of the method, which, from this time forth, enabled us to sterilise infected wounds, whether newly inflicted, or of long standing. In communications made

I Dakin, Presse Médicale, loco citato.

2 Carrel, Dakin, Daufresne, Dehelly, and Dumas, Presse Médicale, Oct. I1, 1915.

${ }^{3}$ Carrel, Archives de Médecine et de Pharmacie Militaires, May, 1916.

4 Pozzi, Académie de Médecine et Societé de Chirurgie, 1915 and 1916. 
to the Société de Chirurgie, M. Tuffier ${ }^{1}$ pointed out the results which it was possible to obtain by the same technique. Le médecin principal Uffoltz published in 1916 (January) an important article, ${ }^{2}$ upon results obtained in hospitals under his control, by MM. Perret, Dupuy, Lemaire, Hornus, Perrin, Vigne, Moyroud, etc. About the same date, M. Pozzi communicated in our name to the Académie de Médecine observations demonstrating that sterilisation allowed wounds to be closed, in many cases, after a period varying from four to ten days. On March 28th, M. Pozzi read a paper on a report by M. Uffoltz concerning the secondary union of wounds. On April I Ith, M. Perret read before the Académie de Médecine a paper in which he announced the results obtained by the sterilisation of wounds in wards under his care. Not one of II I cases had suppurated. On May 2nd, one of us read a paper at the Académie de Médecine on the subject of 153 wounds treated in the hospital at Compiègne during the month of December, 1915. Of these 155 wounds, 135 were closed. Of the I 35 successes, I 2 I were united before the I 2 th day.

On May 23rd, M. Quénu demonstrated at the Société de Chirurgie the results obtained by MM. Hornus and Perrin in one of the field hospitals under the charge of M. Uffoltz. These surgeons had treated I2 I wounds in their wards. In 44 cases they had been able to suture, and the other 77 cases were ready to be sutured at the moment of M. Quénu's visit. At the same meeting M. Tuffier read a paper by MM. Dehelly and Dumas on the sterilisation and closure of wounds

1 Tuffier, Societé de Chirurgie, 1915 and 1916.

2 Uffoltz, Archives de Médecine et de Pharmacie Militaires, Jan. 1916. 
in war. In 31 out of 33 cases, union by first intention had been obtained after secondary closure.

Although these results showed, in an unmistakable manner, that infected wounds could be sterilised and sutured, yet even when more than a year had elapsed since the technique had been made known, the chemical sterilisation of wounds was still an exceptional occurrence. It was only at the hospital at Compiègne, at M. Depage's hospital at Panne, and in two or three territorial hospitals that the method was employed in its integrity.

Nevertheless, the employment of this method was steadily extending, thanks to M. Pozzi, M. Tuffier, M. Regaud, and a few surgeons of other nationalities. In Belgium M. Depage, ${ }^{1}$ from the beginning of 1916 , succeeded in accomplishing the sterilisation and secondary closure of a large number of wounds, some of which were accompanied by fracture. In Paris Señor Chutro, of Buenos Ayres, succeeded in sterilising and healing old infections of the bones and articulations. In New York Mr. Gibson and Mr. Lyle began to apply the method of chemical sterilisation in ordinary surgical practice.

At the close of $1916 \mathrm{Mr}$. Sherman, ${ }^{2}$ principal surgeon to the United States Steel Corporation, demonstrated that the chemical sterilisation of wounds constitutes a considerable improvement in the treatment of industrial casualties.

The possibility of maintaining wounds in a sterile condition enables us to study the laws of cicatrisation

'Depage, C. R. Societte de Chirurgie, 1916, p. 1987, and Feb. 1917.

2 Sherman, Surgery, Gynecology and Olstetrics, January, 1917. 
with an exactness hitherto unknown. A comparison of the curves of sterilisation and of cicatrisation shows us in an exact manner how the various procedures employed in the treatment of wounds act upon those wounds. Similar technical methods were employed by Professor Halsteed in the ingenious experiments which were carried out under his supervision at the John Hopkins Hospital.

In the treatment of mastoiditis $\mathrm{M}$. Mahu ${ }^{1}$ systematically sterilised the wound immediately after operation, reducing the duration of the treatment to one-third of what it was previously. MM. Tuffier and Depage ${ }^{2}$ treated purulent pleurisies by sterilising the pleural cavity and suturing the opening. They thus succeeded in very greatly diminishing the duration of the treatment.

From September, 1916, we investigated more particularly the application of the method to the wounds of men already infected when they reached the hospitals in the rear. These researches were undertaken by MM. Guillot and Woimant, in the Compiègne hospital. They demonstrated the fact that suppurating wounds and old fractures can be rendered sterile, and that complications resulting in the death of the patient or the amputation of the limb may be almost entirely avoided.

Suppuration might have been suppressed in the hospitals as early as September, 1915. But our methods encountered such opposition on the part of certain gentlemen who were at the head of the medical profession in France, that they were scarcely anywhere

1 Mahu, Presse Medicale, 1917.

2 Tuffier et Depage, C.R., Société de Chirurgie, r9r7. 


\section{2 TREATMENT OF INFECTED WOUNDS}

applied. A perusal of the reports of the Societé de Chirurgie and the Académie de Médecine shows with what culpable frivolity a method which might have saved the lives and limbs of large numbers of wounded men was rejected. ${ }^{1}$ Those who criticised us so severely ${ }^{2}$ had not taken the trouble either to examine our technical methods or to check our results. They knew nothing whatever of the methods which they were criticising. ${ }^{3}$ Their responsibility is all the greater inas-

1 “. . . et nous apporter cela d'Amérique, laissez-moi rire. . .." M. Broca, professeur à la Faculté de Médecine de Paris, Jan. 5th, 1916. Bulletin et Mémoires de la Société de Chirurgie de Paris, vol. xlii. pp. 104 and 105.

2 Especially should be noticed the communications made in 1915 and I916 to the Société de Chirurgie de Paris or at the Réunion chirurgicale de la IVe Armée, by MM. Delbet, Hartmann, Broca, Potherat, Chaput, etc. Bulletin et Mémoires de la Société de Chirurgie and Presse Médicale, 1915-1916.

${ }_{3}$ With regard to the attitude of the Sociéte de Chirurgie, note M. Pozzi's communication to the meeting of May 17, 1916.

“.... In any case, one should see for oneself at Compiègne-only two hours from Paris-the condition of the wounded treated by the new method. This is what I have already done myself, on two occasions, when I took part in the memorable discussions which took place at the meetings of our Society, the $5^{\text {th }}$ and 26 th of January; and at the Académie de Médecine, the IIth of January last. And it is what I have begged my colleagues to do, at once. I am glad that, at last, M. Quénu has followed my advice. The time has come at last to repair the real injustice committed towards a method which for long months past had displayed in vain evidence of its value, which is certainly destined to save, in the future (as it has already done in the immediate past), a great number of wounded men, and to lessen, in almost every case, the gravity of mutilations and infirmities.

"In fact, after the sentence, almost of reproof, pronounced against it here, and at the Académie de Médecine in the month of January last, there was brought about amongst the young surgeons at the front a sudden hesitation to apply the new method, which at first they had received with marked approval. This was emphatically to be regretted. Further-may I be allowed to say in all sincerity?-it is not only the wounded to whom 
much as their position in the University of France and the hospitals of Paris lent weight to their verdict.

The aim of this book is to show how surgical sterilisation of the greater number of infected wounds may be obtained. In the following pages we shall describe the principles which allow a given antiseptic to act in an efficient manner. The application of these principles constitutes a "method," that is to say, an entity, no portion of which should be altered at random. The deplorable results obtained in several hospitals by surgeons who believed they were using our methods, but who, in reality, were altering them according to their fancy, make clear the necessity for observing exactly the directions which will be laid down in the following pages. The best way to learn the method is to see it applied. Hence this book is especially intended to recall essential details of the technique to those who already know something of its application.

this attitude of our great learned Societies risks doing harm, but it is the Societies themselves, who, $d$ priori, and without relying upon an investigation easy to carry out (l'hôpital Carrel being at Compiègne), have publicly refused to the treatment newly instituted the merit of originality and of progress...." 


\section{CHAPTER I}

THE PRINCIPLES OF THE TECHNIQUE

DESTRUCTION by chemical means of the micro-organisms infecting a wound is rendered possible by the different resistances presented by the tissues equipped with a circulation, and the microbes which are found on their surface. The idea must be grasped that a given antiseptic substance, applied at a certain concentration, and during a certain time, is able to destroy microbes without damaging the normal tissues to any appreciable extent. The chemiotherapy of wounds is easier to realise than that of the blood. In the latter case a substance capable of destroying microbes is harmful to the corpuscles, because the resistance of isolated anatomical elements is but little different from that of microorganisms.

The mere application of an energetic antiseptic substance, by any form of technique whatsoever, cannot be relied upon to sterilise a wound. The success of the method which enables us to render aseptic an infected wound is not due to the marvellous properties of a new drug. It should rather be attributed to a combination of means, which enables us to make use of a definite antiseptic substance, under such conditions of concentration and duration that its action becomes efficacious. This 
method is a combination of which each single part is essential to the rest. The antiseptic cannot be altered without changing the manner of using it. In the same way a modification of the technique demands an antiseptic endowed with different chemical properties.

The technique of sterilisation has been studied, not by a series of experiments in vitro, but actually upon the wounds themselves. While tracing the bacteriological evolution of a wound we have determined the conditions under which a given antiseptic is capable of bringing about rapidly the total disappearance of microbes. By this means we have determined that a substance powerfully bactericidal, yet only slightly irritating, such as Dakin's hypochlorite of soda, will sterilise a wound if it remain in contact with the microbes during a known period of time and at a certain degree of concentration. As the wound responds to treatment in becoming sterile, and as the progress of the sterilisation cannot be gauged by a mere clinical examination, the bacteriological study of the secretions is the guide needed for therapeusis.

The method, therefore, is based upon the employment, rigorously controlled by the microscope, of an approved agent, under conditions of contact, of concentration, and of duration, established by direct experiment upon infected wounds.

\section{The Choice of AN Antiseptic}

In order to choose the fittest substance to sterilise an infected wound we must consider, apart from its bactericidal action, many other factors, such as its capacity 


\section{I6 TREATMENT OF INFECTED WOUNDS}

for irritating the tissues, its toxicity, its solubility, its power of penetrating the tissues, and of being absorbed by them, and the manner in which it reacts with proteids and other constituents of the tissues. The destruction of bacteria under the influence of a chemical agent is due to the reaction of the antiseptic on the one side, with, on the other, proteins and other substances which enter into the constitution of micro-organisms. Suspended in water, microbes are easily destroyed by antiseptics, because the mixture contains no other proteins. But when they are immersed in blood serum, pus, or other exudations, their destruction is more difficult, because the antiseptic acts not only on the micro-organisms but also on the protein substances in the midst of which they are found. That is precisely why the value of a substance intended for the treatment of wounds, should be judged according to its bactericidal action on microbes in suspension in blood-serum and pus, and not upon microbes simply suspended in water.

The bactericidal activity of all known antiseptics is greatly reduced by the presence of blood-serum or analogous substances. This reduction is so great in certain cases that the substance employed under these conditions practically loses all its value. Dakin and Daufresne have shown, in the experiments described later on, the enormous diminution in bactericidal power of certain antiseptics under the action of blood-serum. In these experiments the antiseptic action of substances was estimated by the degree of concentration of the solution capable of destroying in two hours, at the temperature of the laboratory, microbes in suspension in water and in horse-serum. 
The technique followed by Daufresne was as follows : A series of tubes was prepared containing 5 cubic centimetres of a solution of the substance of a degree of concentration progressively diminishing. To each tube was added one or two drops of a culture (twenty-four hours in peptonised bouillon) of the organism to be studied. A control-tube was at the same time prepared containing 5 c.c. of distilled water with one drop of the culture. The mixtures of antiseptic and microbes were carefully shaken every half-hour, and kept at a temperature of $18^{\circ}$ to $20^{\circ} \mathrm{C}$. for two hours. Afterwards a loopful of the liquid from each tube was placed in each of a series of tubes, each containing 3 c.c. of bouillon. These tubes were placed in the incubator for twentyfour hours, and kept at a temperature of $37^{\circ} \mathrm{C}$. When, at the end of twenty-four hours, there was no development, it was decided that the degree of concentration of the antiseptic was sufficient to kill the organism. Incomplete sterilisation was indicated by the growth of the organism in the bouillon. Examination of the antiseptic action of the substance in presence of blood-serum was made in a similar manner, but to the liquid contained in the first series of tubes were added 5 c.c. of horseserum, previously warmed to a temperature of $55^{\circ}$ or $56^{\circ} \mathrm{C}$.

In this fashion some two hundred substances were studied by Dakin and Daufresne. The micro-organisms which were used as tests were staphylococci, streptococci, the bacillus pyocyaneus, and the bacillus of Welch. In the following table will be found the results of some of Daufresne's experiments, made by means of a fresh culture of staphylococci on certain antiseptics in daily 


\section{I8 TREATMENT OF INFECTED WOUNDS}

use. The sign + indicates that the culture is positive, and the sign - that it remained sterile.

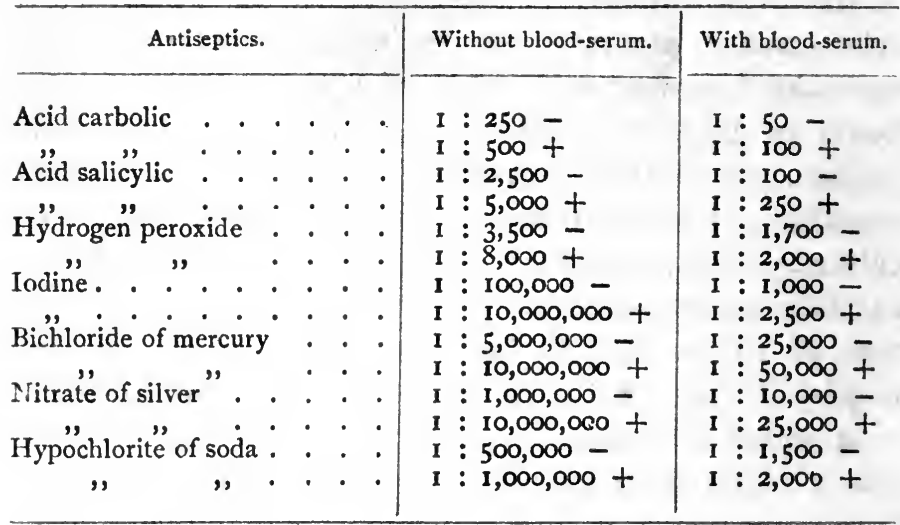

This table shows what feeble power is possessed by an antiseptic which has had a great vogue-carbolic acid. It also demonstrates that bichloride of mercury, which has only a mediocre action on an infected wound, nevertheless kills the staphylococcus in presence of bloodserum of $I: 25,000$. These experiments clearly show us that in the choice of a suitable antiseptic many qualities beside bactericidal action have to be considered; it has been demonstrated that bichloride of mercury, nitrate of silver, and iodine, which have a high germicidal potency, are nevertheless the least suitable for wound treatment. Therefore it is well to become acquainted with the practical inconveniences of the substances we are about to examine.

Phenol has a very poor bactericidal power, especially when acting in the presence of blood-serum. If employed in concentration sufficient to render efficient its 


\section{THE PRINCIPLES OF THE TECHNIQUE I9}

germicidal action, it becomes highly destructive to normal tissues.

Hydr" peroxide solution gives encouraging results wt gactericidal action is examined in a testtube. L. wounds, on the contrary, it has a very feeble action, because it decomposes with the greatest readiness under the influence of the catalysis always going on in the tissues and in the blood corpuscles. Consequently, its action is only exerted during a comparatively insignificant period of time. The mechanical detergent action which results from the rapid disengagement of oxygen when in contact with infected surfaces, has probably a greater value than the antiseptic action of the hydrogen peroxide itself. Dakin ${ }^{1}$ quotes on this subject an interesting experiment which had been communicated to him by Prof. E. K. Dunham of New York. A rabbit which had received an intra-venous injection of Welch's bacillus (Bacillus aërogenes capsulatus or Bacillus perfringens) was killed. The infected liver was cut up very carefully into tiny fragments. Placed in the incubator with hydrogen peroxide solution, it was found that the volume of a fragment of infected liver must not exceed a millimetre cube, if the micro-organisms contained in it were to be killed. Should the fragments be a little larger, the bacilli of Welch multiplied actively. Hydrogen peroxide, therefore, may be considered as having but a feeble antiseptic action, even against anaërobic microbes.

Bichloride of mercury readily loses the greater part of its antiseptic power in presence of pus and the substances of which the tissues are made. Besides, it is very irritating, even in dilute solution.

${ }^{1}$ Dakin, Presse Médicale, 1915. 
Nitrate of silver has a greater value than bichloride of mercury. But it becomes irritating when used in sufficiently strong solution. Many substances which enter into the composition of the tissues inhibit its action in a marked manner. The sensitiveness to light of silver compounds is also an objection to their use.

Iodine, so valuable for sterilisation of the skin, has yielded results much less satisfactory when applied to the disinfection of deep wounds, because it coagulates proteins and irritates the tissues. The penetrative power of iodine is feeble. Treated by this substance, wounds continue to suppurate, and heal more slowly than the rest.

Hypochlorite of soda has a high germicidal power and many other useful qualities. But the hypochlorite of soda found in commerce has an extremely variable composition. Besides, it contains free alkali, and often free chlorine. Consequently, it is irritating when applied to a wound.

The deleterious action of antiseptic solutions upon living tissues should be studied as carefully as their bactericidal action. It is, in fact, absolutely necessary that the substance should be tolerated by the tissues during a prolonged period. The disfavour with which the antiseptic method is regarded by the majority of surgeons is partly due to the use of destructive substances, such as carbolic acid or corrosive sublimate, which have done harm without sterilising the wounds.

In a series of experiments which he made with Mme. Carrel in M. Tuffier's laboratory at the Hôpital Beaujon, Dakin studied the action of a great number of substances on connective tissue. The experiments were made on guinea-pigs. Small fragments of sponge of 


\section{THE PRINCIPLES OF THE TECHNIQUE 2I}

similar weight were placed under the skin of the abdominal wall by means of a short incision which was immediately closed by a suture. On one side, by means of a hypodermic syringe, I c.c. of the substance to be studied was injected. In the sponge placed on the other side, which served as a control, I c.c. of physiological saline solution was injected. At the end of fortyeight hours examination of the region showed a thickening, more or less considerable, of the tissues surrounding the fragment of sponge which had received the solution. By the change in volume of the sponge, the action of the substance employed upon connective tissue could be estimated. In this manner carbolic acid, iodine, bichloride of mercury produced marked tumefaction. The animals injected with bichloride of mercury usually died rapidly. Those injected with carbolic acid showed extensive necrosis of the abdominal wall.

It was only after having made the comparative examination of a large number of substances, from the point of view of their bactericidal action and of their irritating action upon normal tissues, that Dakin decided upon neutralised hypochlorite of soda and chloramines.

A. Dakin's Hypochlorite of Soda. - The antiseptic properties of hypochlorite of soda have been known for a very long time. ${ }^{1}$ But it is not possible to use hypochlorite, either in the form of eau de Javel or of Labarraque's solution, for the sterilisation of wounds, because these solutions are irritating, and may cause grave injury to the tissues. Because of this, Dakin endeavoured to lessen the irritant qualities of the hypochlorites without modifying their antiseptic action.

${ }^{2}$ Dakin, British Medical Fournal, 1915, p. 809. 
The principles of the preparation of the hypochlorite solution by Dakin are as follows. A solution of hypochlorite of soda almost always contains free alkali, even when it is prepared with the greatest care. Though looked upon as neutral, it has an alkaline reaction. This reaction is due not only to the alkali which may arise from the mode of preparation, but also to a hydrolytic dissociation of the hypochlorite which produces free soda and hypochlorous acid.

The amount of this dissociation has been measured by Duyk, and is quite considerable. It is to the formation of free alkali, therefore, that the irritating action of hypochlorite is due. The amount of the hydrolytic dissociation increases with the dilution, so that, from a practical point of view, a hypochlorite cannot be rendered non-irritant by merely reducing its concentration. Really, a point is soon reached at which the bactericidal action is lessened, while the irritating properties of the solution remain. Besides these two sources of free alkali, it must not be forgotten that soda may be liberated by the action of hypochlorite on proteins. A reaction takes place, in which the chlorine of the hypochlorite attaches itself to the nitrogen of the proteins, as will be demonstrated later.

Dakin, for the neutralisation of the alkali of the hypochlorite of soda, made use of the following known facts. Blood and other organic liquids, and also certain artificial saline solutions containing mixtures of polybasic acids, such as phosphoric acid, are able to keep their essential neutrality, even after the addition of acid or alkali. This phenomenon is due to the fact that the addition of acid or alkali simply changes the relative proportion of two or 
more salts of the polybasic acid present in the solution. Setting out from this principle, and employing a feeble polybasic acid (boric acid), Dakin succeeded in preparing a simple mixture of hypochlorites, which remains very nearly neutral under all conditions, and which in consequence does not irritate the tissues. This solution contains a mixture of hypochlorite and polyborate of soda and small quantities of free hypochlorous acid and boric acid. In this manner the irritating action of caustic soda is avoided. In fact, if alkali should form, it would be immediately neutralised by the boric acid and the acid borates present in the solution.

Dakin's hypochlorite differs from eau de Javel and Labarraque's solution in that its destructive action upon the tissues is very slight. Study of the communications made to the learned Societies of Paris, and particularly to the Académie de Médecine, shows that the necessity for using a non-caustic antiseptic has not been grasped. Surgeons do not yet comprehend that Dakin's solution, containing no free alkali, can be employed under conditions where the use of eau de Javel or Labarraque's solution would be absolutely impossible.

A simple experiment made by Daufresne in the laboratories at Compiègne will show the essential difference which exists between Dakin's solution, on the one hand, and eau de Javel and Labarraque's solution on the other. In three tubes there were placed Dakin's solution, eau de Javel, and Labarraque's liquor. The strength of the three solutions in hypochlorite of soda had previously been brought to 0.5 per cent. A fragment of skin from a still-born infant was placed in each of the three tubes. At the end of two hours the action on 


\section{TREATMENT OF INFECTED WOUNDS}

the skin of eau de Javel and Labarraque's solution was already manifest. The skin was greatly swollen, and the slightest friction could detach the epidermis in a fragile pellicle. In the hours following the process continued, the fragment became completely transparent, and after twelve hours in eau de Javel, and fourteen hours in Labarraque's solution, the fragment of skin was completely dissociated. The tubes contained only a powdery sediment. The piece of skin placed in Dakin's solution behaved in quite a different manner. After two hours of contact the epidermis was still very adherent, and the aspect of the skin was normal. At the end of twenty-four hours the alteration in the tissues resembled that observed after two hours' contact with the solutions of Javel and Labarraque.

This experiment shows in a very clear manner the profound difference which exists, from the biological point of view, between Dakin's solution and the nonneutralised hypochlorites. In a word, Dakin's researches allow us to use to-day hypochlorite of soda under conditions such that it will sterilise the tissues without danger to them. We shall see, later, that the hypochlorite only kills the microbes when its action is extended over a long period, and it is of a strength of 0.5 per cent. (about), conditions impossible to realise if the solution be caustic.

It is possible to obtain a solution even less irritating than Dakin's solution, if we prepare it by the electrolytic method. Electrolytic hypochlorite has not hitherto been employed in surgery on account of its defective keeping properties. As it is completely devoid of alkali, its concentration diminishes very rapidly, and in a few 


\section{THE PRINCIPLES OF THE TECHNIQUE 25}

hours its bactericidal power may become insufficient. In the course of experiments carried out in the Compiègne laboratories, Dakin discovered that electrolytic hypochlorite, that is, hypochlorite completely free from alkali, may be rendered stable by a small quantity of permanganate of potash or silicate of soda. Hypochlorite thus prepared will keep for several weeks without any appreciable loss of concentration. It is less caustic than the chemically prepared solution. Its power of
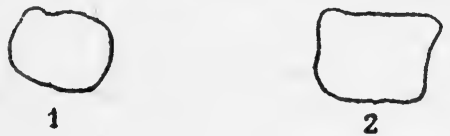

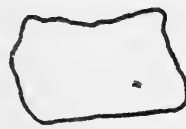

3

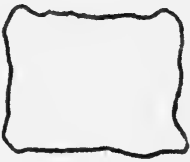

4

FIG. 1.-Four fragments of skin of identical dimensions were placed in chloramine (4), hypochlorite of magnesium (3), Dakin's hypochlorite (r), and electrolytic hypochlorite (2). Results at the end of 48 hours.

dissolving necrosed tissues was compared with that of Dakin's solution by placing, in the two solutions, fragments of skin of identical dimensions (Fig. I). The skin was destroyed more rapidly in Dakin's solution than in the electrolytic solution. Nevertheless, the solvent power of the electrolytic hypochlorite was sufficient to rid wounds of the necrosed tissues which occurred upon their surfaces.

Many other preparations of hypochlorites have been previously employed in surgery. Eau de Javel and 
Labarraque's solution are well known, as well as the hypochlorites of calcium, potassium, and magnesium. Since the beginning of this war, eau de Javel has been used with good results. In November, 1914, in the hospital at Dunkirk, MM. Landry and Jacomet made successful use of eau de Javel in "gas" infections.

But eau de Javel is dangerous, for its strength in hypochlorite is variable in the extreme, and it always contains an amount of alkali which renders it caustic. The alkali contained in eau de Javel and in Dakin's solution when badly prepared is responsible for the secondary hæmorrhages which have sometimes been observed as a result of their employment in the neighbourhood of the blood-vessels.

Hypochlorite of magnesium has been employed with success by M. Dubard. Its bactericidal power is similar to that of sodium hypochlorite, and it is not irritating. Dakin, however, elected to employ the hypochlorite of sodium rather than the magnesium salt, because the solvent power of the former, in the case of mortified tissues, is greatly superior to that of the latter, and because the efficacy of an antiseptic depends to a certain extent upon its ability to cleanse the surfaces of wounds. Daufresne placed fragments of skin of equal dimensions in solutions of magnesium hypochlorite and sodium hypochlorite. Another fragment of skin, placed in chloramine, served as a "control." While the fragment of skin immersed in Dakin's solution shrank to about one-third of its original size, the fragment placed in the magnesium solution underwent a very much less considerable change (Fig. I).

Mixtures of powdered chloride of lime and boric 
acid have been employed by Vincent, Lumière, and Lorrain Smith. The local production, by an admixture of powdered hypochlorites, of hypochlorous acid or chlorine in a comparatively high degree of concentration, is more dangerous to the healthy tissues than the continued application of a weak and neutral solution of hypochlorite of sodium. Moreover, a solution possesses the advantage of penetrating into all the recesses of deep wounds. Vincent's powder, injected into anfractuous wounds, falls upon the coagulum, but does not reach the surface of the wound. It may also form upon the surface of the tissues a crust which protects the bacteria from the antiseptic substance. In this manner serious infections may develop subsequently to the application of Vincent's powder to deep wounds. Moreover, it is impossible in one operation to introduce into a wound a sufficient quantity of antiseptic to sterilise it. To achieve this end the concentration of the active substance would require to be very great, and, consequently, dangerous to the tissues.

Generally speaking, the experiments we have carried out with Dakin, by means of powdered substances, and substances dissolved in fatty matters, such as vaseline or lanoline, have yielded results greatly inferior to those of the experiments made with watery solutions :

1. Bactericidal Action of Dakin's Solution.-The bactericidal action of Dakin's solution of hypochlorite of soda has been studied by Daufresne, using micro-organisms suspended in water, and in water with the addition of horse-serum. Staphylococci in suspension in water are killed in two hours by hypochlorite of a strength of I : 500,000 to I: $1,000,000$; whilst, in the presence of 
horse-serum, the concentration increases, and should be $I: I, 500$ to $I: 2,000$. Streptococci are killed more rapidly. $B$. pyocyaneus in suspension in water is killed in two hours by a strength of 1 : 100,000 to $1: 1,000,000$; whilst in presence of horse-serum a strength of $I: 2,500$ to $I: 5,000$ becomes necessary. In the experiments made on mixtures of pus and hypochlorite, it is found that sterilisation generally takes place when two or three volumes of hypochlorite to one volume of pus are used. The action of hypochlorite naturally varies according to

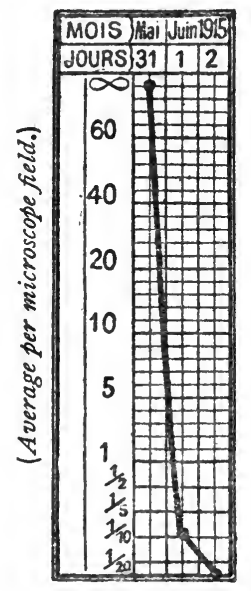

FIG. 2.-Disappearance of the microbes of a highly infected wound, after treatment by Dakin's hypochlorite, May 3rst to June 2nd, 1915. (Case 28.)

$(\infty$ denotes infinity. Translator.)

\section{the character of the pus.}

The results of these experiments in vitro are of but slight importance, for experimental conditions vary too greatly from the actual. In wounds, in fact, a small quantity of pus is found in contact with a large quantity of antiseptic, because the solution of hypochlorite is constantly being renewed. In the experiments in vitro, the duration of the action of the hypochlorite upon the microbes in suspension in the pus is short. If, at the end of two or three hours, one tests for the hypochlorite contained in the mixture, sometimes it is found that it has completely disappeared. The hypochlorite, in fact, rapidly enters into combination with the proteins of the pus, and chemical analysis is no longer able to discover it. It 
is precisely because of this rapid disappearance of hypochlorite when in contact with secretions, that Dakin's solution should be continuously instilled into wounds, or if intermittently, at short intervals. Ignorance of this chemical property of the hypochlorites has led surgeons to be surprised that mixtures of pus and hypochlorite kept in the incubator for several hours should become favourable breeding-grounds for microbes. It is quite evident that, after being treated in this manner, the mixture contains hypochlorite no longer. When a solution of hypochlorite is employed, it is essential to determine the quantity of the active substance contained in the solution before and after the test.

The bactericidal action of Dakin's hypochlorite was next studied in infected wounds themselves. When hypochlorite of soda is applied to a wound in such a manner that its degree of concentration remains constant, and the duration of the application is prolonged, the microbes disappear (Fig. 2). This fact has been observed a very great number of times. Indeed, one might affirm that it constantly happens when intimate contact is established between the antiseptic solution and the organisms. The sterilisation of wounds treated by Dakin's solution is an established fact. But it will be well to enquire if the treatment is actually the determining cause of the sterilisation, and if this sterilisation is due to the hypochlorite of soda.

(a) It might be suggested that, in our observations, the wounds grew sterile spontaneously. In truth this is hardly likely, because one never sees a series of infected wounds become sterile in a few days. Nevertheless, this hypothesis was submitted to experimental 
analysis. Choosing a wound whose various regions were uniformly infected, a square of filter-paper was placed on a selected spot, and kept constantly moist with hypochlorite of soda. On another spot was placed a square of filter-paper of the same size. Then the wound was again covered with a protective dressing. At the end of twenty-four hours, below the filter-paper moistened with hypochlorite, a smooth surface of red granulations was to be seen, and the microbes had completely disappeared. Under the filter-paper which had not been wetted with hypochlorite, the granulations were irregular and pale, and the microbes as numerous as before (Fig. 3). In the portions of the wound which had not been covered with filter-paper, there was no change in the quantity of microbes.

In a case where the half of a wound was dressed with hypochlorite, and the other half with vaselin, there was complete disappearance of microbes in the region treated with hypochlorite, whilst the infection remained elsewhere.

Similar results were obtained with deep wounds. Two shell fragments had penetrated two neighbouring points in the lumbar region; the two fragments were removed at the same time. One of the wounds was treated by the continuous instillation of hypochlorite, and the other by a simple dressing. The wound treated remained painless, and the microbes disappeared completely from the smears; whilst the wound not treated became painful, was surrounded by a red aureola, and was the seat of streptococcal infection. In the seton type of wounds, we could often observe that the region where the hypochlorite penetrated was sterile, while the 


\section{THE PRINCIPLES OF THE TECHNIQUE 3 I}

portion where the hypochlorite did not penetrate still held a great number of microbes. Numerous similar observations showed in a very distinct fashion that the

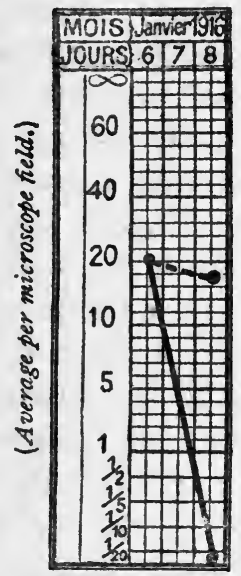

FIG. 3.-Superficial wound of left arm. Comparative action on an infected wound of pieces of filterpaper soaked or not in hypochlorite of soda. The continuous line represents the diminution of the microbes from 20 to o per field of the microscope, and the dotted line, the condition of the "control " portion of the wound. (Case No. 247.)

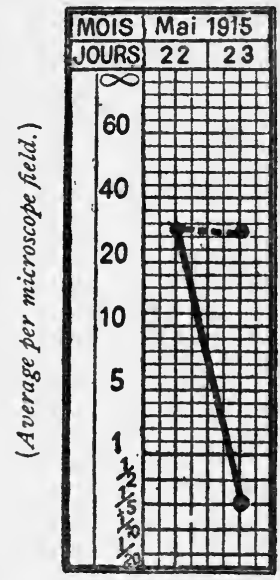

FIG. 4. -Superficial wounds of the left thigh. Comparative study of the influence of Dakin's hypochlorite and of physiological saline solution. Two wounds equally infected and of the same man were treated, one by hypochlorite, the other by saline solution. These two wounds contained from 20 to 30 microbes per microscope field. The continuous line represents the diminution in number of microbes in 24 hours under the influence of hypochlorite. The dotted line represents the state of the wound treated by saline solution at the end of the same time. (Case No. 52.)

relation of cause and effect existed between the treatment employed and the results obtained.

(b) Next, it must be made clear whether the result is due to the antiseptic action of the hypochlorite, or to the mechanical action of the instilled liquid. The 
following experiments were devised to elucidate this point. A wounded man had upon his thigh two wounds of dimensions almost identical, and with bacteriological conditions practically the same. One was dressed with hypochlorite and the other with physiological saline solution. At the end of twenty-four hours, the surface of the wound dressed with hypochlorite did not show a single microbe per field, while the wound treated by saline solution had more than thirty microbes per field (Fig. 4). Other experiments were made by means of wounds, on the surfaces of which were applied squares of filter-paper of similar dimensions. One of the squares carried solution of hypochlorite, while the other had physiological saline solution. At the end of twenty-four hours, the region situated under the hypochlorite contained no microbes, while the region treated by saline solution had a large number. Similarly, observations were made on deep wounds with old lesions. A case of fracture of the femur with great loss of substance and extensive osteo-myelitis of the bony extremities, was observed for several months. An india-rubber tube introduced into the suppurating cavity permitted the instillation, during arranged periods, of hypochlorite, or of hypertonic saline solution. When the case was having the hypochlorite, the pus contained many microbes and had no smell. When the hypertonic saline solution was substituted for the hypochlorite, the pus immediately gave out a tainted odour, and the microbes became much more numerous. As soon as the hypochlorite was again instilled, the odour disappeared, and the number of microbes diminished. Similar experiments were made several times with like results. 
In another series of experiments the wounds were irrigated either by distilled water or by Wright's hypertonic solution. The wounds had previously been sterilised. After some hours' instillation the bacterial curve rose. Distilled water and the hypertonic solution produced similar results (Figs. 5 and 6). It was enough to sub-

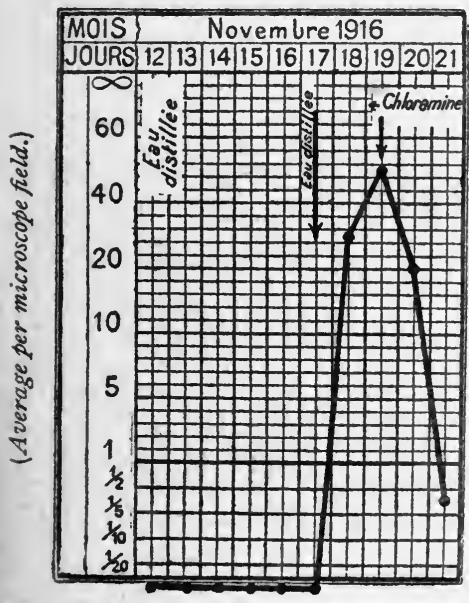

Fig. 5.-Reinfection of a wound dressed for 24 hours with distilled water, (The wound is dressed with distilled water on the I7th; chloramine is applied on the 18 th. $-T r$.)

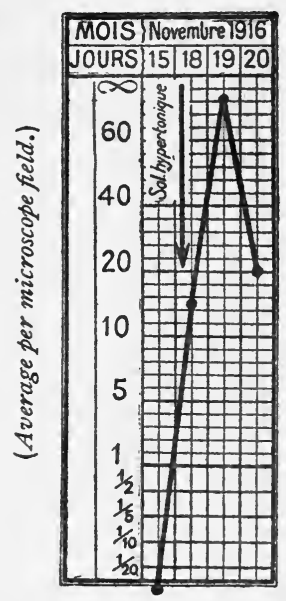

FIG. 6.-Reinfection of a wound dressed for 24 hours with hypertonic solution. (The hypertonic solution was applied on the 17 th; Dakin's solution on the 18 th. $-T r$.)

stitute Dakin's solution for the hypertonic solution or the distilled water, and the curve descended, while the wound once more became aseptic. This experiment was frequently repeated, always yielding identical results.

The action of M. Delbet's chloride of magnesium and of Dakin's solution were investigated under precisely similar conditions. The curve in Fig. 7 shows that a sterile wound whose surface was treated by the 


\section{TREATMENT OF INFECTED WOUNDS}

instillation of chloride of magnesium became rapidly reinfected. The number of microbes quickly became innumerable. An attempt was made to assist the action of the chloride of magnesium by washing the wound daily with neutral oleate of soda. The number of microbes diminished, but did not fall to zero. Directly the oleate of soda was discontinued the number of microbes again became innumerable. At this point the

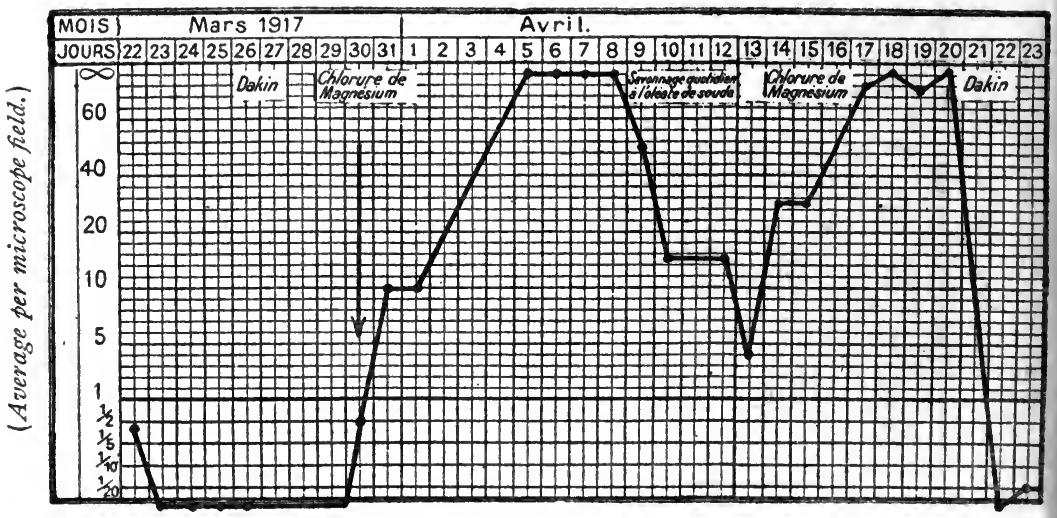

FIG. 7.--Intense infection of a wound treated with chloride of magnesium, afterwards sterilised by means of Dakin's solution

chloride of magnesium was replaced by Dakin's solution, and the number of microbes immediately fell to zero, as the curve indicates.

These experiments show that distilled water, Wright's hypertonic solution, or Delbet's chloride of magnesium permit of the rapid reinfection of wounds previously aseptic, and that sterilisation is effected immediately these substances are replaced by an identical volume of hypochlorite of soda. It is therefore evident that the 
hypochlorite acts by virtue of its antiseptic power, not in a mechanical manner.

(c) The antiseptic power of hypochlorite is not due to its alkalinity. In M. Tissot's paper, read at l'Académie des Sciences by M. Dastre, ${ }^{1}$ that author attributed the actiōn of hypochlorite of soda upon wounds to its alkalinity, and declared that the treatment to which Dakin had submitted it had the result of enfeebling its power! Although M. Tissot furnished in support of his opinion no precise observations, we have made experiments to test if the presence of an alkaline substance on the surface of a wound had any influence upon its bacteriological condition.

Upon a large surface wound on the external aspect of a limb, two squares of filter-paper of equal dimensions were placed. One of the squares was moistened with physiological saline solution, and the other with a solution of carbonate of soda, 0.5 per cent. Two days afterwards it was found that the number of microbes under the paper moistened with saline solution was almost identical with the number under the paper moistened with carbonate of soda. This experiment was repeated on other wounds with similar results. The alkaline solution had no more effect on the microbes present at the surface of a wound than had physiological saline solution.

2. Action of Hypochlorite on Microbial Toxins.-This point was considered in a course of experiments made by $M$. Auguste Lumière. ${ }^{2}$ In a case of grave tetanus, he took some cubic centimetres of pus from a highly infected wound of the leg. This pus was divided into

1 Tissot, C. R. Académie des Sciences, Sept. 13, 1915.

${ }^{2}$ Auguste Lumière, C. R. Académie des Sciences, March 6, 1916. 


\section{TREATMENT OF INFECTED WOUNDS}

two equal parts, of which one was brought to double its volume by the addition of I per cent. solution of hypochlorite, and the other brought to the same volume by the addition of chloride of sodium solution, .0 .8 per cent. After the lapse of an hour, I c.c. of each of these preparations was injected into guinea-pigs. It was found that the animals which had received the "control" pus died from tetanus in eight or ten days, whilst those in which had been injected pus with the addition of hypochlorite presented no symptoms of tetanus and survived.

This experiment was repeated with pus containing various microbes, streptococci, staphylococci, perfringens, etc. These preparations were administered to guineapigs by subcutaneous injection and to rabbits by intravenous. It was demonstrated that pus containing hypochlorite gave reactions either slight or benign, while the purulent fluids without added antiseptic produced evidences of infection, often ending in death.

M. Lumière, in another series of experiments, candlefiltered pus both treated and not treated with hypochlorite, and injected animals with the filtrates. Filtrates of pus treated with hypochlorite produced no change in condition of the animals, while the filtrates from the control pus provoked pyrexia and emaciation. In short, these filtration products, placed in contact in vitro with leucocytes and microbes, demonstrate that phagocytosis is much more active when the pus has been treated with hypochlorite.

M. Lumiere's experiments prove, therefore, that hypochlorite of soda destroys toxins contained in pus. This destruction of toxins by oxidising antiseptics plays a 
favourable part in sterilisation, either in allowing phagocytosis to become effectual, or in preventing the impregnation of the organism by noxious substances. Perhaps it explains, in part at least, the rapid disappearance of general symptoms, presented by patients suffering from extensive suppuration, when their wounds are treated by Dakin's solution.

3. Toxicity of Dakin's Solution.-Hypochlorite of soda is very slightly toxic to the organism, when it is injected on the surface of wounds, or, in animals, into the subcutaneous cellular tissue. We have injected under the skin of the abdominal wall of guinea-pigs quantities of antiseptic relatively considerable, without unfavourable result. For example, three guinea-pigs weighed respectively 565 grammes, 570 grammes, and 510 grammes. These had respectively 8 c.c., II 4 c.c., and 12.75 c.c. of Dakin's solution, that is to say, $1 / 70,1 / 50$, and $1 / 40$ of their body-weight. They presented no abnormal symptom, and remained in good health.

Hypochlorite of soda, which is harmless when subcutaneously injected, is very dangerous if injected into the general circulation. An injection of ten cubic centimetres into the marginal vein of the ear of a large rabbit rapidly caused death. Hypochlorite of soda is strongly haemolytic, and therefore should never be injected into veins. Indeed, it is prudent never to inject it under pressure into deep wounds, in order that it may not be absorbed by the tissues. Amongst the numerous cases of wounded men treated by hypochlorite of soda, we have never had accidents which could be attributed to a toxic action of this substance.

4. Aotion of Hypochlorite on the Tissues.-Experiments 


\section{TREATMNNT OF INFECTED WOUNDS}

made upon guinea-pigs had already shown that a small quantity of hypochlorite of soda injected into a fragment of sponge placed under the skin, produced no modification of the tissues obvious to clinical examination. Further, Dakin's solution, instilled for several days, sometimes several weeks, over the surface of a wound, in a general way set up no marked irritation. It was already evident from these observations that Dakin's hypochlorite had little action upon the tissues. However, more exact experiments were initiated, in order the better to determine the action of the antiseptic on dead tissues, on isolated anatomical elements, and on tissues deprived of circulation.

(a) Action of Hypochlorite on Tissues deprived of Circulation.-When fragments of skin from a guinea-pig or a human foetus are placed in Dakin's solution, the tissues begin to disintegrate at the end of twenty-four hours. The same is true of fragments of mortified tissues. This action is less marked if electrolytic hypochlorite be employed. On the surfaces of wounds mortified tissue dissolves, as a rule, in five or six days; but occasionally fragments of tendons or aponeuroses are left adhering to the tissues, and persist for a longer period. Dakin's solution appears incapable of sterilising a fragment of necrosed tissue on the surface of a wound. In such a fragment bacteria would find a safe refuge. In the treatment of wounds, therefore, it is indispensable to employ a substance capable of dissolving tissues deprived of circulation. On the other hand, if the wall of a blood-vessel is mortified, the slough falls off more rapidly than if the wound is left to itself. Similarly, the clots which often obliterate vascular wounds are enabled 
to dissolve under the action of hypochlorite. For this reason we must carefully examine the condition of the vessels at the moment of surgical intervention, and must effect an accurate preventive haemostasis.

(b) Action of Hypochlorite on Isolated Anatomical Elements. - If blood or pus is placed in a tube containing an excess of hypochlorite, the red corpuscles are rapidly attacked. When the volume of hypochlorite is sufficient the anatomical elements are completely disintegrated. However, in the secretions of wounds treated by hypochlorite the polynuclear cells are not much altered, and often contain microbes. It is probable that phagocytosis occurs in the walls of the wound, out of reach of the antiseptic substance, which remains on the surface of the tissues. Generally speaking, there is no need to accomplish the dissolution of those leucocytes which have absorbed micro-organisms; but in certain cases the complete destruction of the phagocytes is of importance. This conception is unknown to the French surgeons, who generally speaking confuse the chemiotherapy of local infections with that of general infections.

In the chemiotherapy of general infections the antiseptic substance should be injected into the circulatory system; it must not, accordingly, exert an injurious action upon the leucocytes. If, indeed, it were to prevent phagocytosis it would deprive the organism of one of its most valuable means of defence.

In the chemiotherapy of local infections the importance of the leucocytes is of a totally different nature. Indeed, as the bactericidal substance does not sink into the depth of the tissues, its action is exerted only on the surface of the wound. The leucocytes existing in 


\section{TREATMENT OF INFECTED WOUNDS}

the wall of the wound are able to continue their functions, even if the bactericidal substance is injurious to them. As for the leucocytes which occur on the surface of the tissues, they are replaced by a chemical substance whose bactericidal action is much more powerful than their own. On the other hand, large numbers of these leucocytes, having phagocytosed the microbes, are still living, and may once again enter into the tissues and there spread the infection. The researches of Rous ${ }^{1}$ have demonstrated that the red corpuscles or the pathogenic phagocytosed microbes are protected by the wall of the leucocytes from the humours of the organism and from bactericidal chemical substances. It is thus important to destroy, on the surface of wounds, the leucocytes which are loaded with bacteria, as well as the pus bacteria. The so-called cytophylactic medication of a local infection is based on ignorance of the protective rôle which the leucocytes fulfil in respect of the microbes. It may produce disastrous results, above all in cases of cerebral wounds. Indeed, we find in the secretions of such wounds leucocytes which are loaded with microbes, which remain included in the cerebral substance at the moment of cicatrisation. It is probable that the protection offered to the microbes by the phagocytes which have absorbed them enables them to produce secondary retractile cicatrices, or encephalitis, or a cerebral abscess.

This is why we must employ a substance capable of destroying all the anatomical elements deprived of circulation. The concentration of Dakin's solution is such that it enables us to utilise the different degrees of

${ }^{1}$ Rous and Jones, Fournal of Experimental Medicine, 1916, p. 601. 
resistance presented, on the one hand, by the microbes, the free anatomical elements, and the necrosed tissues; and, on the other hand, by the normal tissues provided with circulation. It destroys the former and respects the latter.

(c) Action of Hypochlorite on Tissues equipped with a Circulation.-In order to appreciate the action of Dakin's solution on living tissues we have investigated the progress of cicatrisation of wounds treated with hypochlorite.

Certain technical difficulties are presented by this research. It is essentially necessary that the conditions of the wounds whose healing is being studied, and particularly their microbial state, should not vary throughout the duration of the experiments. Should these conditions vary, one may no longer attribute to the substance employed the eventual modifications in the progress of cicatrisation. Furthermore, the surface of wounds, in spite of the irregularity of their outline, must be measured exactly.

Up to the present, no one has taken the trouble to study in any precise manner the factors capable of modifying the rapidity of cicatrisation. The bacteriological condition of wounds the subject of experiment has not hitherto been taken into account. It is recognised, however, that the presence of microbes on the surface of a wound has a profound effect on the progress of repair. In all the forms of technique hitherto employed, this truly important omission destroys the value of all the experiments on and observations of substances supposed to aid cicatrisation. This error in technique explains the contradictions found in all medical 


\section{2 TREATMENT OF INFECTED WOUNDS}

publications on the subject of topical applications in the treatment of wounds. Every surgeon attributes a power more or less marvellous to some substance which the surgeon of the next hospital looks on as insignificant.

In the same way, estimation of the progress of cicatrisation has always been left to individual opinion. As a matter of fact, it has never been sought to devise a technique which would permit exact measurement of the surface of a wound, with estimation in square centimetres of the amount by which it lessens day by day. The ignorance we manifest, after so many ages of surgical practice, of the real influence of the substances used in treating wounds, is only due to the absence of scientific method. To obtain exact data on this subject, it was necessary in the first place to experiment on wounds placed under conditions which remain unchanged throughout the duration of the observations, and afterwards to devise a method which would allow the progress of cicatrisation to be measured.

(a) The Conditions of the Wounds.-The wound must be that of a man immobilised in bed, and whose general state does not vary during the period of observation. The bacteriological state of the wound plays an important part in the progress of cicatrisation. Rapidity of repair varies according to the nature and the volume of the infection. When microbes are allowed to multiply on the surface of the wound, it is impossible to know if the modifications of cicatrisation are due to direct action of the substance experimented with upon the tissues, or to a favourable or unfavourable action of this substance on the microbial flora; or to the algebraic sum of the two causes. Therefore the daily control of 
THE PRINCIPLES OF THE TECHNIQUE 43

the state of the wound, by means of the microscope, is indispensable, to avoid a false interpretation of the experimental results.

The experiments were made on surface wounds, and sometimes on deep wounds. Wounds of regular perimeter were preferred to those whose margins were torn. Wounds of elongated form were specially chosen, so that one half could be treated by a substance, while the other half served as a control. Or, better still, wounds of nearly equal size were used, situated in the corresponding region in the same individual One of the wounds was dressed with a substance to be tested, while the other served as a control.

Every day the bacteriological condition of the wound was examined by the aid of "smears," and sometimes of cultures. As microbes were found, steps were taken to eliminate them. The granulating surface and the neighbouring skin were washed carefully with neutral oleate of soda. Then the granulations were sterilised by means of hypochlorite of soda or chloramine. When the bacteriological examination showed that sterilisation was complete, the wound was dressed, either with oleate of soda, or with stearate of soda containing small quantities of antiseptic, or with vaselin, or saline solution. In this manner it was possible to keep wounds almost completely aseptic. The daily bacteriological examination allowed reappearance of the infection to be discovered, and allowance to be made for it in the interpretation of the experiments. On wounds thus prepared the action of the substances was studied.

(b) Technique of the Measurement of Wounds.-In most cases the progress of repair was studied on surface 


\section{TREATMENT OF INFECTED WOUNDS}

wounds, and only rarely in deep wounds. The surface of a wound was measured in the following manner. A sheet of thin celluloid was applied over the surface of the wound. By the aid of a pencil (used for marking glass) the outline of the epithelial margin was traced, and in every case where it was possible, the contour of the cicatrix at its union with sound skin. The drawing thus obtained was transferred to a sheet of ordinary paper. Then, with the aid of a planimeter, the area of the wound, properly so called, was measured, also that of the surface of the wound increased by that of the cicatrix. Thus in square centimetres was obtained the area of the two surfaces, and, by subtracting the first from the second, the area of the surface of cicatricial tissue was obtained. When a deep wound was in question, its capacity was obtained by filling it with water and so measuring the volume.

Graphic representation of the cicatrisation of a wound was obtained in the following manner. Time was represented in abscissae and surface in ordinates. Curves were thus obtained which enabled one, day by day, to estimate the variations of the surface and those of the cicatrix. Thus the parts taken by connective and epithelial tissues in repair may be observed.

The investigations of Carrel have shown that the curve of cicatrisation thus established is geometrical in form. Lecomte de Nouy has recently determined the algebraic expression of this curve. ${ }^{1}$ Nouy's formula consists of two equations-

1 Carrel, Fournal of the American Medical Association, 1910, and Fournal of Experimental Medicine, 1916. Lecomte du Nouy, Fournal of Experimental Medicine, Nov, 1916. A. Hartmann, Thise de Paris, 1916. 
THE PRINCIPLES OF THE TECHNIQUE 45

$$
\begin{gathered}
\frac{s-s^{\prime}}{s} \\
\frac{s \sqrt{\mathrm{T}}}{t+s^{\prime}\left(\mathrm{I}-i\left(t^{\prime}+\sqrt{\left.\mathrm{T}+t^{\prime}\right)}\right)\right.}=i
\end{gathered}
$$

$s$ is the total surface of the wound on the day of commencing the observation ;

$s^{\prime}$ is the surface of the wound $t$ days later, at the time of the second observation (usually four days later, so that the cicatrised area $s-s^{\prime}$ shall be sufficiently important);

$t$ is the time elapsing between the two first observations ;

$\mathrm{T}$ is the "age" of the wound, reckoned from the time of the first observation $s$; so that in the first equation $\mathrm{T}=t$;

$t^{\prime}$ is the time which must elapse between the day of the last observation $s^{\prime}$, and the day for which it is desired to calculate the theoretical area of the wound ;

$i$ is a coefficient characteristic of each wound, on the determination of which the formula is based.

Thus we see, by the mere examination of the two equalities ( 1 ) and (2), that the former tends to establish a certain index of cicatrisation $i$, which is then introduced into the latter, in order to calculate the surface of the wound at any given time.

The index $i$ is obtained by means of the formula (I) after two observations made at four days' interval when the wound is aseptic. Experiment has shown that this index is a function of the age of the wounded man, and also of the surface of the wound, and that it is larger in proportion as the latter is smaller, and the patient 


\section{TREATMENT OF INFECTED WOUNDS}

younger. Lecomte du Nouy establishes a curve which makes the previous calculation of the index unnecessary (Fig. 8). The only elements needed for the calculation of this curve of cicatrisation are the area of the sterile wound and the age of the patient.

By means of two observations made at four days' interval, or even by means of a single observation, the age of the patient being known, it was possible to calculate the theoretical curve of cicatrisation. On the

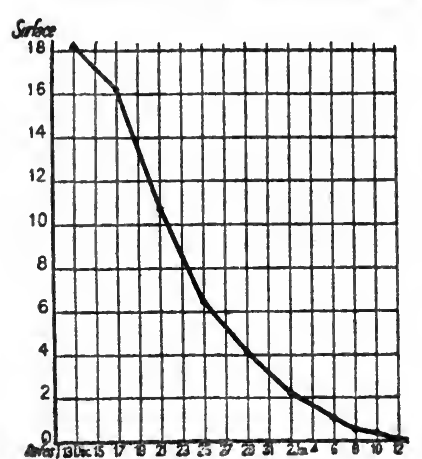

FIG. 8.-Cicatrisation curve of an aseptic wound. Surface, expressed in square centimetres, forms the ordinates, whilst time, in days, the

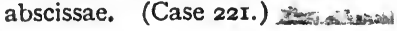

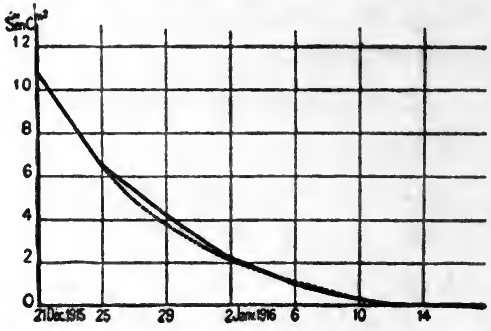

FIG. 9.-Curves, observed and calculated, for the same wound. By means of observations made the 17th and 2ist of Dec., the progress of cicatrisation was calculated according to the formula of Lecomte du Nouy. A continuous line represents the observed curve, and a dotted line the calculated curve. The coincidence of the two curves is almost perfect. (Case 221.)

same sheet the course of the observed curve was plotted (Fig. 9). It was thus possible to ascertain the abnormal variations of the rate of repair to be observed in the wound, these manifesting themselves by the divergence of the observed curve from the calculated curve.

As we possessed also the chart of the bacteriological condition of the wound, it was easy to estimate almost exactly the part played in the progress of repair by the substance under examination. By means of this method 
the action of hypochlorite of soda upon the repair of wounds was examined. Experiments were successively made upon infected wounds, and upon those surgically sterile, that is to say, wounds whose secretions examined by means of "smears" no longer contained microbes.

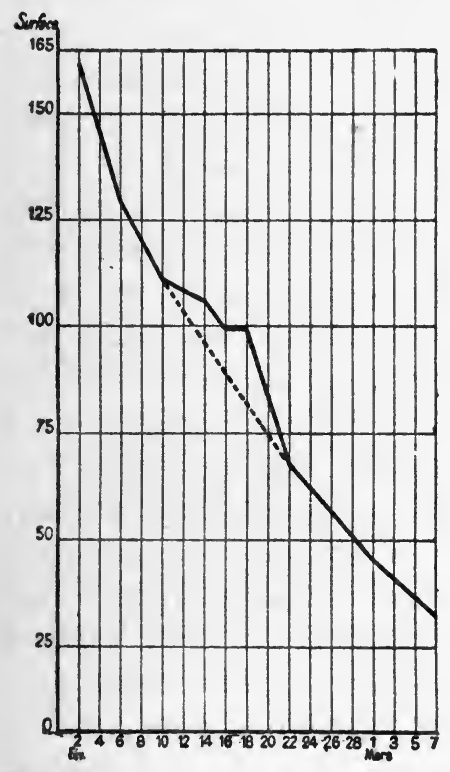

FIG. IO.-Cicatrisation curve of a wound of the abdominal wall. Slowing down of cicatrisation from Feb. Ioth to Feb. 18th, due to a re-infection. Acceleration from Feb. I8th to Feb. 22nd, under the influence of Dakin's solution. (Case 327.)

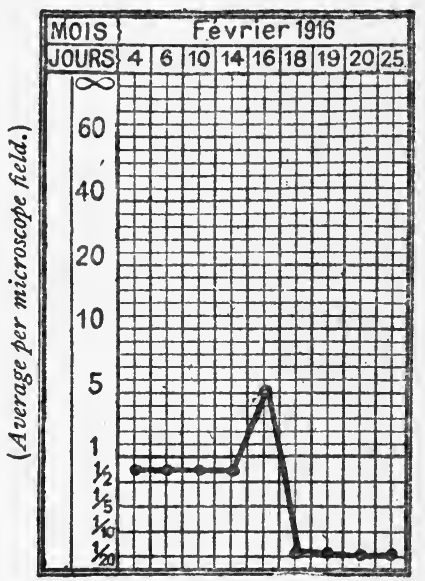

FIG. II.-Curve indicating the bacteriological condition of the preceding wound from $\mathrm{Feb}$. 4th to Feb. 21st. The slowing down of cicatrisation coincides with a re-infection of the wound which reached its maximum Feb. I6th, and the acceleration coincides with the sterilisation which occurred Feb. 18th. (Case 327.)

(c) Action of Hypochlorite upon the Cicatrisation of an Infected Wound.-Many experiments were made upon surface-wounds whose curves of cicatrisation were known, and of which the bacteriological condition was 
recorded. These wounds generally showed from five to twenty microbes per microscope field, and the observed curve of cicatrisation showed a slighter fall than the calculated curve (Figs. Io and II). A perforated tube was applied to the surface of the wound and Dakin's solution instilled every two hours. In all cases without exception cicatrisation was hastened and the curve of cicatrisation dropped (Figs. 12 and 13 ). The speed of the repair often increased in such a manner that the observed curve rejoined the calculated curve, but without ever having a more rapid fall than that of an aseptic wound. Therefore there was no accelerating action due to the hypochlorite.

The rapidity of the cicatrisation in presence of Dakin's solution was sometimes considerable. A wound of the leg, wide and of long standing, communicating with an unsterilised bone injury, yielding a great number of microbes from its surface, was healing very slowly. The cicatrisation curve was dropping only slightly. This wound had a surface of 75 square centimetres. As soon as it was treated with Dakin's solution the curve fell sharply. In four days the wound lessened by 28 square centimetres, and during the following days the repair continued at approximately the same rate. It should be noticed that the sterilisation chart showed at the same time a considerable lessening in the number of microbes. The same phenomenon was observed in all wounds uniformly infected, and cicatrising with a known rapidity, which were treated with Dakin's solution. With the exception of those containing a foreign body, all wounds responded to the treatment. To remove the foreign body was to ensure that the wound would follow the general rule. 


\section{THE PRINCIPLES OF THE TECHNIQUE}

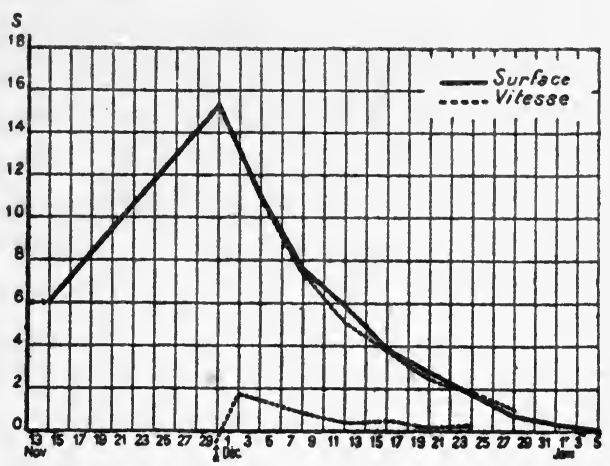

FIG. 12.-Influence of the sterilisation of a wound on the progress of cicatrisation.. The cicatrisation curve shows how a sluggish-looking and highly infected wound steadily enlarged from 6 to 15 square centimetres from the I $4^{\text {th }}$ to the 29th of Nov. It was sterilised on the 29th of Nov. Instantly cicatrisation commenced and its course followed a geometric curve.

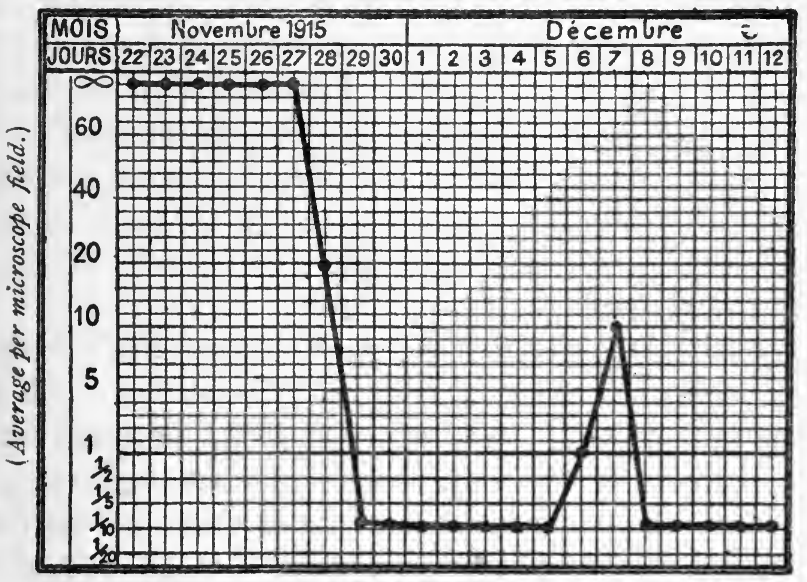

FIG, 13.-Bacteriological curve of the preceding wound. The graph shows that, under the influence of Dakin's hypochlorite, the number of microbes, at first infinite, rapidly dropped. The coincidence between the date of sterilisation of the wound and that of the beginning of normal cicatrigation should be noticed. 


\section{TREATMENT OF INFECTED WOUNDS}

Upon deep wounds few observations were made. However, some experiments were carried out of the following type. A collection of pus had formed on the antero-external aspect of the leg of a man with arthritis of the knee. This collection, which was accompanied by a rise of temperature, was opened at its upper part and the pus evacuated. The next day the wound was washed with Ringer's solution, and its capacity measured 26 c.c. The wound was irrigated with hypochlorite. Twenty-four hours later the suppuration had disappeared. In the bottom of the cavity a little liquid was found, syrupy, yellow, transparent. The secretions contained only one coccus per microscope field. The volume of the cavity now was not more than 7 c.c. Forty-eight hours later it was reduced to 2 c.c., and the wound was completely sterile. Then it closed. In short, an abscess cavity of 26 c.c. was sterilised and completely closed in four days. Similar experiments were made, and yielded results comparable. But the diminution in volume of deep wounds comes about in a more irregular manner than the cicatrisation of surface-wounds. It was upon the latter, therefore, that the majority of the experiments were made.

In order to obtain more strictly controlled observations, experiments were made on different parts of the same wound. For example, two strips of filter-paper were applied at the upper and lower extremities of a wound of the external aspect of the arm, with fracture. Each strip stretched from one margin of the wound to the other, over the granulations, after the manner of a bridge. The previous bacteriological examination had shown that the whole surface of the wound was 
uniformly infected. The filter-paper at the lower part of the wound received an instillation of Dakin's hypochlorite every two hours, whilst the filter-paper at the upper part was not moistened (Fig. 14 and Fig. 15).

At the end of three days it was obvious that the edges of the wound were not altered in the upper region, but that, in the lower part of the wound, cicatrisation

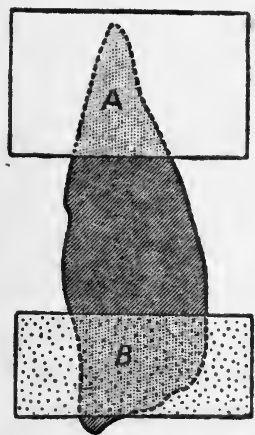

FIG. 14.-Influence of hypochlorite on an infected wound. Wound on the external aspect of the arm presenting an infection of cutaneous origin. A, control filter-paper. B, filterpaper soaked in Dakin's hypochlorite.

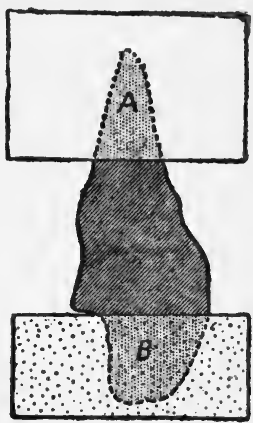

FIG. I5.-The same wound three days later. The lessening of the wound at the level of the control paper A is of the slightest. Beneath the filter-paper $B$, the influence of the hypochlorite is manifest ; the epithelial margin has greatly advanced, and the wound has lessened in a well-marked manner.

had progressed much more quickly. The parts covered by filter-paper moistened with hypochlorite showed granulations softer and redder than those in the other regions of the wound. The change in the appearance of the granulations followed a transverse line very closely corresponding to the upper border of the filter-paper. A marked acceleration of the cicatrisation, therefore, had 
taken place in the region treated by Dakin's solution (Fig. I5). At the same time the bacteriological examination showed that the microbial flora were not modified over the untreated part of the surface of the wound, whilst in the region covered by filter-paper soaked in hypochlorite, microbes had completely disappeared. In other experiments, where a part of the infected wound was dressed with vaselin, and another part with hypochlorite of soda, there was to be observed, in similar fashion, acceleration of cicatrisation in the region treated by hypochlorite.

There was, therefore, a coincidence between the acceleration of cicatrisation and the application of Dakin's hypochlorite, under certain conditions, to the surface of the wound. We might, therefore, have been tempted to attribute to the hypochlorite of soda a stimulating action on cicatrisation. But as the wounds submitted to experiment were infected, and the bacteriological charts also showed a coincidence between the disappearance of the microbes and the acceleration of cicatrisation, it was probable that the cicatrising influence of the hypochlorite of soda was only apparent. In fact, the following experiments showed that hypochlorite of soda exercises no active influence on wounds already aseptic.

(d) Action of Hypochlorite upon the Cicatrisation of an Aseptic Wound.-In order to keep aseptic wounds sterile while their cicatrisation is being studied, hypochlorite of soda is applied to the surface during periods more or less long. But the rapidity of repair of these aseptic wounds, treated by means of hypochlorite, is not altered, and the curves do not show a more marked fall. This shows that the hypochlorite of soda has no 


\section{THE PRINCIPLES OF THE TECHNIQUE 53}

cicatrising effect, and that the acceleration which it produced in the repair of infected wounds is due simply to the suppression of microbes. Under the actual conditions of the experiments, hypochlorite does not delay the repair of wounds moistened every two hours with 0.5 per cent. Dakin's solution. Or rather, any delay produced by the action of the hypochlorite is too slight to be evident.

We have endeavoured to study this possible retarding action of hypochlorite with the help of a more precise form of technique. On a large wound taking up the external aspect of the arm, repeated bacteriological examinations had shown the absence of microbes. The lower half of the wound was covered with a piece of gauze moistened every two hours with Dakin's solution, whilst the upper half was dressed with vaselin. At the end of four days a tracing of the wound was taken, and, on comparing it with the preceding tracing, it was seen that the epithelial border had progressed a little more rapidly under the vaselin than under the hypochlorite (Fig. 16).

At this time both the upper and lower parts of the wound were still aseptic. It would seem, therefore, that the hypochlorite of soda had slightly retarded the healing of an aseptic wound. But this retarding action was much feebler than the action of certain microbes, as the later history of the experiment showed. The wound was still being dressed with vaselin and hypochlorite. Bacteria soon appeared in the region dressed with vaselin, whilst that covered by hypochlorite remained sterile. A new tracing was taken, and, on comparing it with the preceding, it was found that the rapidity of 


\section{TREATMENT OF INFECTED WOUNDS}

healing had become greater under the hypochlorite than under the vaselin (Fig. 17). When physiological saline solution was used instead of vaselin, similar results were obtained.

These results were checked by experiments in which

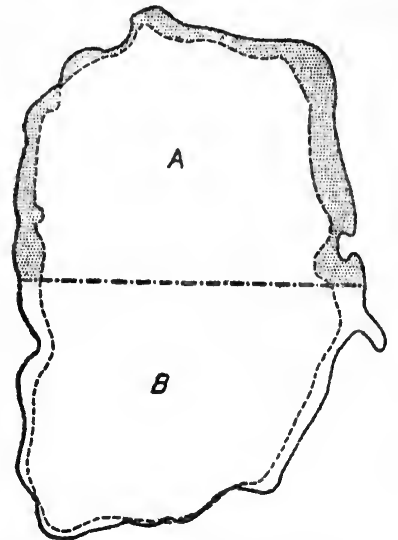

FIG. 16.-Influence of hypochlorite on a sterile wound. The continuous outline represents the contour of a wound of the outer region of the arm which was sterile, Dec. I6. The half A was dressed with vaselin, and the half $B$ with hypochlorite. The dotted line represents the state of the wound Dec. 20. It shows that the part A dressed with vaselin has healed a little more quickly than the part B dressed with hypochlorite.

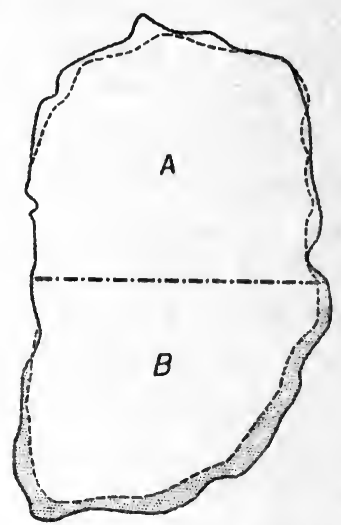

FIG. 17.-Influence of hypochlorite on an infected wound. The preceding wound, under the influence of vaselin, became slightly reinfected. The dressings, however, were continued, the part A with vaselin, the part B with hypochlorite. The continuous outline represents the state of the wound Dec. 20. The dotted line represents the state of the wound Dec. 24. It is seen that the cicatrisation has taken place more rapidly in the part B, dressed with hypochlorite, than in the part $\mathrm{A}$, dressed with vaselin.

the rate of cicatrisation of wounds treated with hypochlorite was compared with that of wounds treated with other substances, in particular by distilled water, Wright's hypertonic solution, M. Delbet's chloride of magnesium, flavine, and chloramine. 
The wounds treated by the instillation of distilled water (Figs. 18 and 19 ) or of Wright's hypertonic solu-

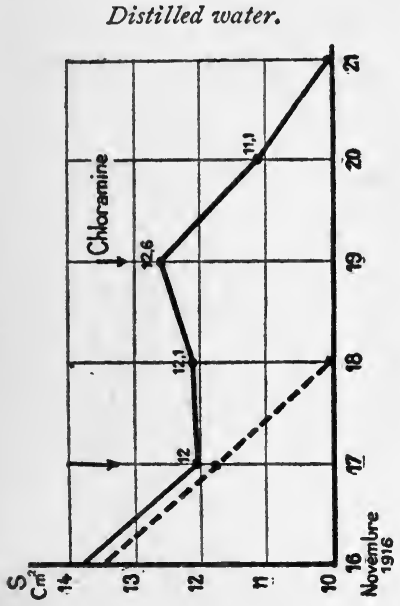

FIG. 18.-Cicatrisation curve of a wound treated with distilled water. The cicatrisation, which was normal, slowed down and came to a full stop. Then the wound became slightly enlarged. The cicatrisation!became normal once more after a dressing with chloramine. There is a great divergence between the calculated curve and the observed curve.

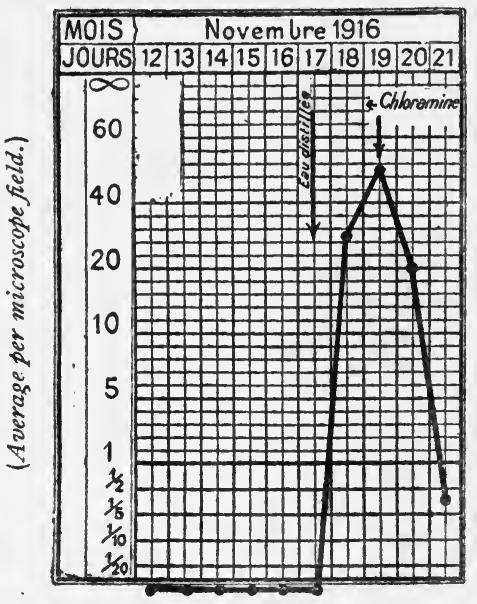

FIG. 19.-Sterilisation curve of the foregoing wound. This curve shows that a reinfection occurred after the application of irrigation with distilled water.

lution (Figs. 20 and $2 \mathrm{I}$ ) behaved in precisely the same manner. The curve of cicatrisation was modified by the first application of the substance employed. The dimi-, nution of the rate of cicatrisation constantly coincided with a reinfection of the wound. The apparent retarding action of the liquid employed was due simply to the rapid reinfection of the wound. This fact is 


\section{TREATMENT OF INFECTED WOUNDS}

clearly demonstrated by a comparison of the curves of cicatrisation and sterilisation.

The cicatrisation of a wound treated with Delbet's chloride of magnesium is strikingly affected. Directly a sterile wound is treated with instillations of chloride of magnesium its curve undergoes modification, tending

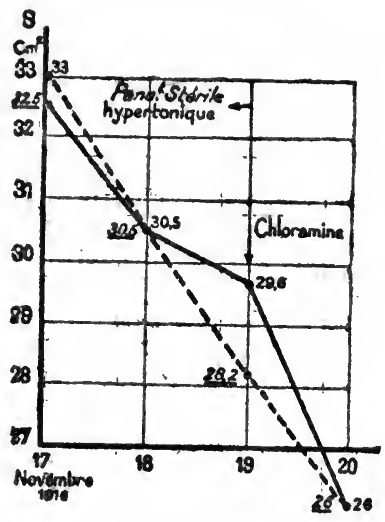

FIG. 20.-Cicatrisation curve of a wound dressed with hypertonic solution.

The observed curve,diverges from the calculated curve after the application of the hypertonic solution, and returns to it as soon as a dressing of chloramine is applied.

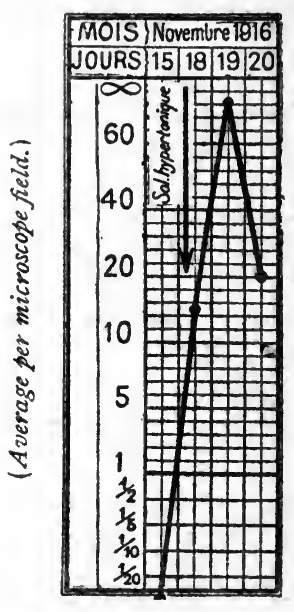

FIG. 2I.-Sterilisation curve of the same wound.

Under the aseptic dressing the wound becomes reinfected; it proceeds towards sterilisation ${ }_{\text {y }}$ as soon as the chloramine is applied.

to approach the horizontal. This indicates a decided retardation of cicatrisation. In the wound whose curve we reproduce below (Fig. 22) the solution of magnesium chloride was replaced, after the lapse of a few days, by hypochlorite of soda. The curve fill once more, the cicatrisation being accelerated. At the same time the 


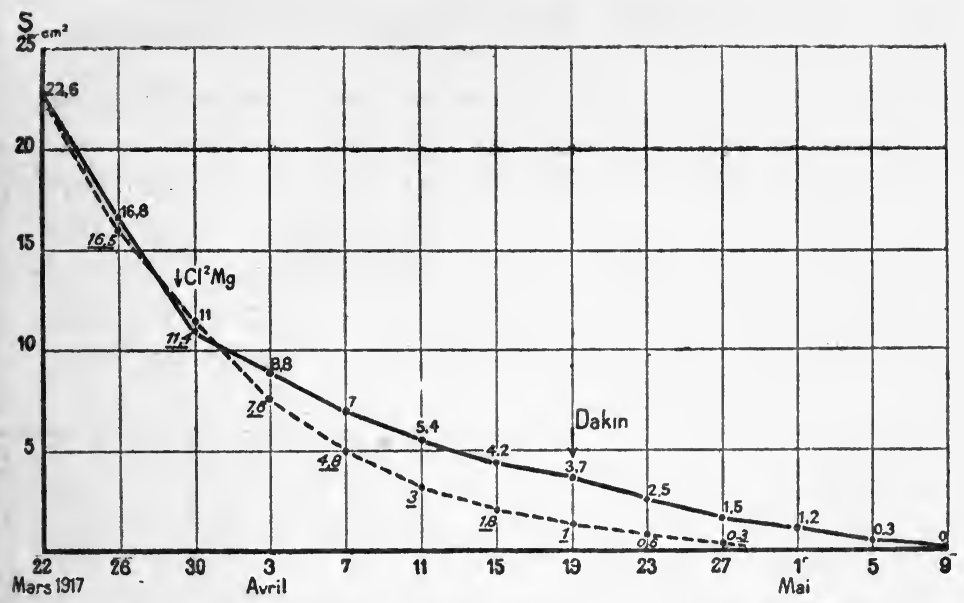

FIG. 22.-Cicatrisation curve of a wound treated with chloride of magnesium.

(Case No. 799.)

From the day when chloride of magnesium is applied to the wound the observed curve diverges rapidly from the calculated curve, showing retarded cicatrisation. From the moment when Dakin's solution is applied the observed curve tends to rejoin the calculated curve.

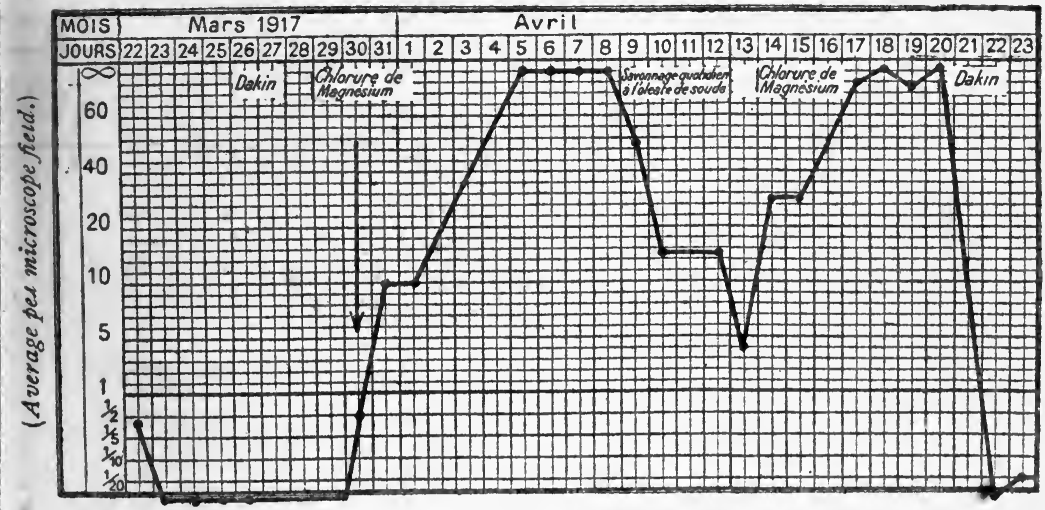

FIG. 23.-Sterilisation curve of a wound treated with chloride of magnesium. (Case No. 799.)

Directly chloride of magnesium is applied to the wound the curve rises, for the number of microbes quickly becomes very great. The lowering of the curve which follows is due to washing the wound with oleate of soda. When the washing is discontinued the number of microbes again becomes innumerable. The last drop of the curve is due to the application of hypochlorite of soda, which results in sterilisation. 
curve of microbic infection showed that the retardation of cicatrisation coincided with a reinfection of the wound, which was covered with vast numbers of microbes (Fig. 23). After the application of hypochlorite of soda the wound again became sterile. This is why the cicatrisation curve tends to become normal again when the use of M. Delbet's solution is discontinued. In these

15
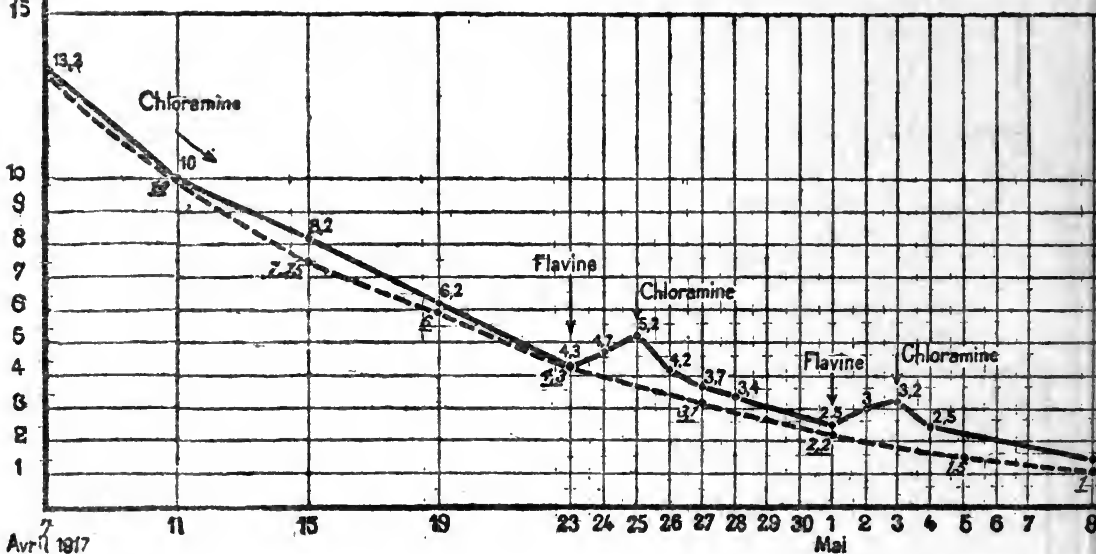

FIG. 24.-Cicatrisation curve of a wound dressed with flavine. Directly flavine is applied the observed curve diverges from the calculated curve, for the wound increases in size. The cicatrisation becomes normal again as soon as chloramine is applied once more. A second experiment upon the same wound produces the same arrest of cicatrisation.

two cases the retarding action of the substances employed was only apparent. The retardation was due in reality to the infection which occurred immediately.

Other substances-for example, Browning's flavineexert a direct action on the process of cicatrisation, as is shown by the curves of cicatrisation and sterilisation. In the case of a patient whose wound was sterile, and undergoing cicatrisation in a normal manner, installations 


\section{TREATMENT OF INFECTED WOUNDS}

Immediately the curve became horizontal and even proceeded to rise, for cicatrisation was not only arrested; it actually retrogressed, and the dimensions of the wound increased (Fig. 24). At the same time the bacterial curve showed that the wound had remained aseptic (Fig. 25). It was therefore obvious that the retrogression of cicatrisation, not being due to infection, was to be attributed to a deleterious action of the substance itself.

When we compare the curves of wounds treated with the aforesaid substances with the curves of wounds

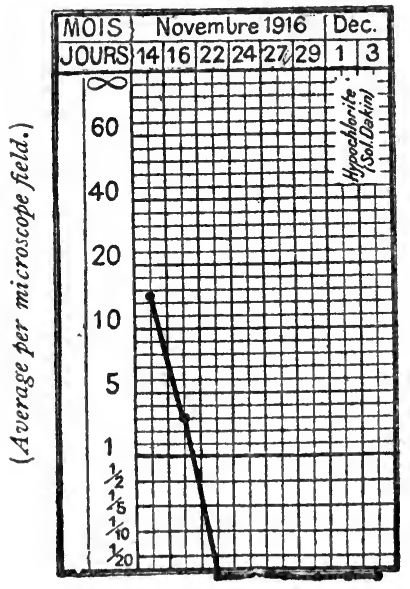

FIG. 27.-Sterilisation curve of the foregoing wound. The wound remains sterile throughout the treatment.

treated with hypocholorite or chloramine, we notice an obvious difference. The wounds treated with hypochlorite (Figs. 26 and 27) or with chloramine (Figs. 28 and 29) become cicatrised according to the cicatrisation curve calculated by du Nouy's formula, with the normal rapidity of aseptic wounds.

In short, in the healing of an infected wound the acceleration produced by hypochlorite is due to its antiseptic power. Hypochlorite does not appear to have any marked action on the tissues in the direction of cicatrisation, when it is used under the conditions of our experiments. Probably it has a slightly retarding effect on the healing of aseptic wounds. But in practice this influence is negligible. 


\section{THE PRINCIPLES OF THE TECHNIQUE 6I}

Dakin's solution, therefore, we may conclude, when applied under suitable conditions, does not in any appreciable manner, harm tissues under repair, which is contrary to the belief of most surgeons.

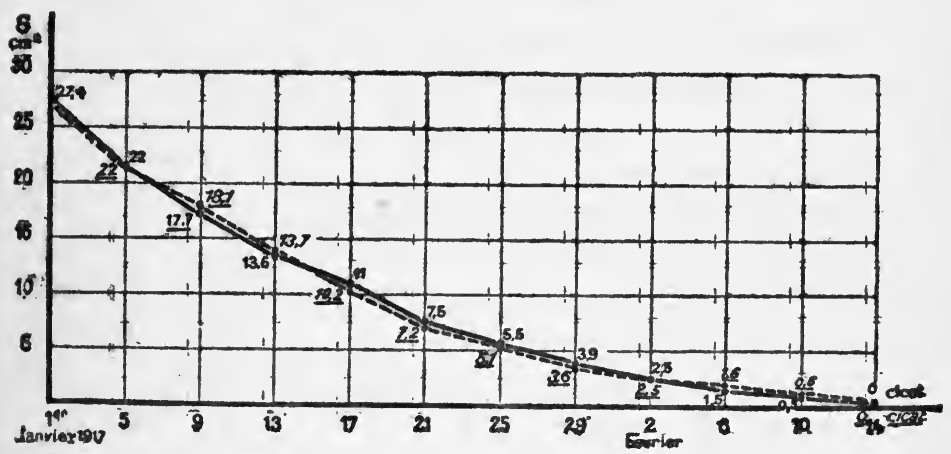

FIG. 28.-Cicatrisation curve of a wound dressed with chloramine (case No. 706). The observed curve coincides exactly with the calculated curve.

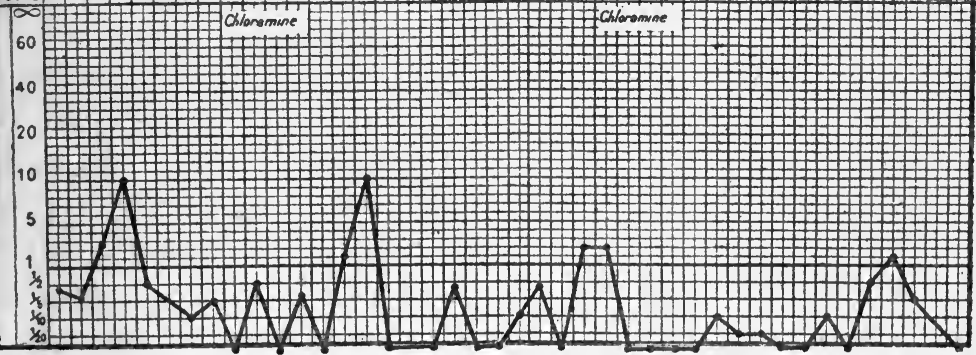

FIG. 29.-Sterilisation curve of a wound treated with chloramine. The wound remains constantly sterile.

5. Action of the Tissues on Hypochlorite of Soda. - The dead or living tissues exert a marked action on the concentration of a solution of hypochlorite. In M. Daufresne's experiment, which we have already described, wherein 
fragments of skin were placed in an excess of Dakin's hypochlorite, it was found, after the lapse of some twentyfour hours, that the concentration of the solution was very greatly diminished. In the experiments conducted by Carrel on wounds of the brain after the death of the animal, it was found that hypochlorite of soda decomposed with very great rapidity. The wound was filled with a solution containing 0.5 per 100 of hypochlorite. At the end of six minutes the solution was withdrawn, and the degree of concentration was found to be only 0.16 per 100. A fresh portion of solution was injected, and withdrawn at the end of seven minutes; its degree of concentration was still 0.23 per 100 . But if a third portion of Dakin's solution was injected into the same cavity, it was found that the degree of concentration had not diminished by more than half at the end of eight minutes. Similar experiments made upon other tissues show that the concentration of a solution of hypochlorite may diminish by 70 per 100 in five minutes.

This destruction of hypochlorite is still more active when the solution is in contact with living tissues. Experiments were made in the peritoneum of the cat or rabbit. A portion of Dakin's solution containing 0.45 per 100 of hypochlorite was poured into a small cup which contained a portion of the epiploön of a cat, which was provided with its normal circulation. At the end of seven minutes the strength of the solution was only 0.07 per 100. A large number of analogous experiments were carried out, and from these it was possible to prove that hypochlorite loses, in a few minutes, when in contact with tissues equipped with their normal circulation, more than four-fifths of its strength. Similar experiments 
THE PRINCIPLES OF THE TECHNIQUE 63

were conducted with wounds in course of cicatrisation. Deep wounds were selected for the purpose, so that the solution could lie in them readily. The wounds whose action on hypochlorite was studied were completely aseptic, or still contained a few microbes. The hypochlorite, whose degree of concentration was known, was poured into the wound, and at intervals of two minutes a few cubic centimetres of the liquid were withdrawn and

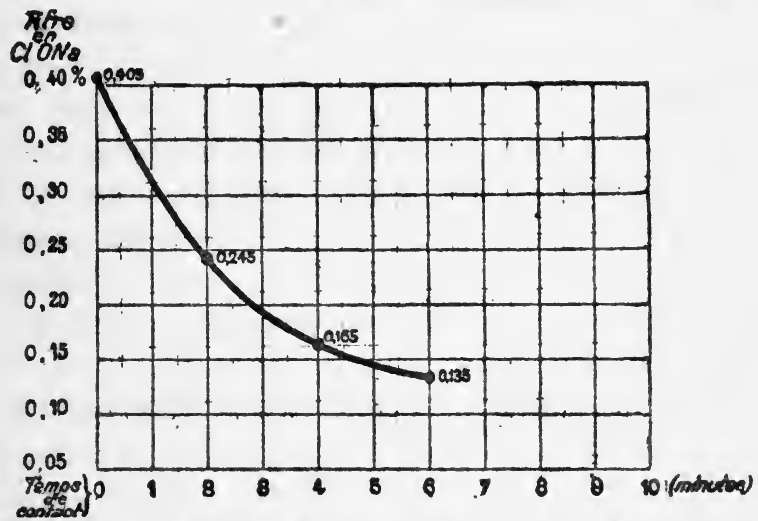

FIG. 30.-Curve showing the rate at which hypochlorite of sodium is destroyed in contact with living tissues.

A 0.4 per cent. solution placed in an almost aseptic wound was reduced to 0.24 per cent. at the end of 2 minutes, and 0.16 per cent. at the end of 9 minutes.

immediately analysed. The results of the experiments were expressed in the form of a curve. In aseptic fractures of the tibia, or fractures which were almost completely aseptic, the strength of the hypochlorite as a rule decreased from $0^{\circ} 4$ to $O^{\circ}{ }_{5}$ in six minutes. At the end of half an hour only 0.05 to 0.06 of hypochlorite was left. In Fig. 30 the curve shows the progressive disappearance of hypochlorite from a solution poured into a wound accompanying a fracture of the tibia. The 
solution, which was 0.40 per 100 at the outset, was 0.27 per I00 two minutes later, and 0.16 per 100 at the end of four minutes. When six minutes had elapsed the strength was reduced to 0.13 per 100 .

The decrease in the strength of the solution of hypochlorite occurs at a variable rate, according to the nature of the tissues, the volume of the secretions, the form of the wound, and many other conditions, which do not permit us to forecast, in the case of a given wound, what will be the rate of destruction of the bactericidal substance. But these experiments show that the tissues exert a manifest action upon the hypochlorite, that the strength of the solution is rapidly modified, and that in order to maintain it at a constant figure fresh quantities must incessantly be supplied to the wound. Hence the necessity of a large quantity of antiseptic solution if we wish to sterilise a wound.

6. Mode of Action of Hypochlorite-Dakin attributes the bactericidal action of the hypochlorites to a chemical reaction similar to that which takes place between ammonia and a hypochlorite, and results in the simplest of the chloramines, as Raschig demonstrated long ago. The destruction of micro-organisms by an antiseptic is due probably to chemical modifications produced in the substances constituting living cells, either by the direct action of the antiseptic, or by the action of products resulting from the combination of the antiseptic with the substances in the midst of which the micro-organisms are found. Amongst the substances contained in living cells and capable of reacting with hypochlorites, proteins play probably the chief rôle. The action of hypochlorites on proteid matters consists, at least in part, in the substitution 
of chlorine for hydrogen in some of the $\mathrm{NH}$ groups, and, afterwards, in the formation of substances belonging to the group of chloramines. Dakin ${ }^{1}$ believes that the property possessed by hypochlorites of attacking proteid matters, forming compounds in which the halogen element is directly attached to the nitrogen, is closely bound up with their bactericidal action.

This hypothesis is supported by the following observations. Free chlorine, bromine, and iodine vary only slightly in their germicidal power. But if the halogen element is converted into the hypochlorite or the hypobromite, a strongly marked difference appears. The bactericidal action of hypochlorites on staphylococci suspended in water, is almost equal to that of free chlorine, whilst that of hypobromites is only equivalent to about one-hundredth of that of free bromine. The bactericidal action of hypoiodite is almost nil. The insignificant bactericidal power of hypobromites and hypoiodites coincides with their feeble capacity for reaction with proteins and amino-acids.

It is also interesting to consider why hypochlorites, which destroy the skin in vitro, leave unharmed living tissues and do not interfere with the healing of wounds.

Soda, it is well known, produces immediate dissolution of the tissues. Fiessinger's experiments, upon the rapidity of dissolution of leucocytes, confirms the fact that the solvent action of the hyposulphites is a function of their soda content. Fiessinger also ascertained that this action diminishes according as hypochlorites containing quantities greater or less of soda are used.

1 Dakin, Cohen, Daufresne, and Kenyon, Proceedings of the Royal Society, 1916. 
Daufresne's experiments, which we have already quoted, demonstrate plainly that Labarraque's solution, which contains free alkali, causes dissolution of skin at a moment when Dakin's solution has not yet produced any perceptible lesion.

Tissues provided with a normal circulation resist perfectly the action of Dakin's solution under the conditions of our experiments. Guillaumin and Vienne attribute $^{1}$ this resistance to the following phenomenon. Take an alkaline solution of such concentration that it hydrolyses and dissolves the fragment of tissue placed in it. If a certain quantity of neutral salt be added, it is known that the tissue can be immersed in the solution thus modified, without its structure becoming altered. Guillaumin and Vienne made the following experiment. Fragments of skin were placed in a 3 per cent. solution of soda, to which had been added 12 per cent. sodium chloride. Other portions of skin were placed in a 3 per cent. solution of soda to serve as controls. The skin immersed in the solution containing chloride remained intact, while the control fragments swelled up and became transparent. However, analysis showed that the same quantity of alkali had been absorbed by the skin in each case. This important process is known in tanneries as "pickling." Guillaumin and Vienne consider it the reason why tissues are not injured by hypochlorite.

Whatever may be the explanation of the resistance of living tissues to hypochlorite, this phenomenon provides a working hypothesis for the chemiotherapy of wounds

1 Guillaumin et Vienne, Archives de Medecine et de Pharmacie militaire, 1916. 
in spite of the destructive action of hypochlorite on proteins.

B. Chloramines.-After having studied the mode of action of hypochlorites, Dakin was led to investigate the substances which act in a manner almost identical, but which are of greater practical value. He believed that the bactericidal action of hypochlorites took place by means of substances formed at the expense of proteins, and containing chlorine in combination with nitrogen. Experiment showed him that when proteins such as blood-serum, white of egg, casein, etc., are treated by hypochlorites, they give rise to products of a high antiseptic potency. These substances, without a doubt, are formed in situ when wounds are treated by hypochlorites. Thus, after the disappearance of free hypochlorite, there still remains in the wound a substance having antiseptic power.

Certain aromatic chloramines which form soluble salts give encouraging clinical results. The best of these compounds are the benzene- or the para-toluene-sodiumsulphochloramines which have been described by Chattaway.
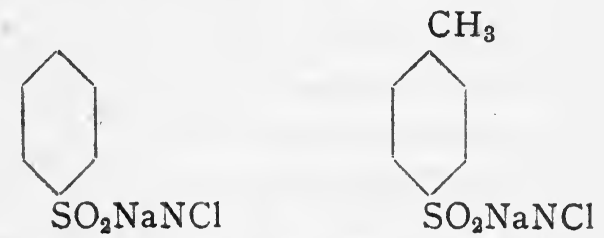

These substances, which possess a very high antiseptic potency, are but slightly irritating, and can be used in a much higher degree of concentration than the hypochlorites. The solutions in general use in our 
experiments vary from 0.2 per cent. to $2^{\circ} \circ$ per cent. The action of these substances is similar to that of the hypochlorites, but their antiseptic potency is superior.

I. Bactericidal Action.-Staphylococci suspended in water are killed in two hours by benzene-sodium-sulphochloramine at a strength of $I: 500,000$, and by para-toluene-sodium-sulphochloramine at a strength of I : $1,000,000$. When horse-serum is present, the strength necessary becomes $I: I, 500$ to I : 2,500 . The B. pyocyaneus, Eberth's bacillus, and the bacillus coli are slightly more resistant than staphylococci, whilst $B$. perfringens and streptococci are more easily killed.

On infected wounds chloramines give results similar to those of hypochlorite of soda. Their action on microbes has been determined in the course of a great number of experiments similar to those we have described when on the subject of hypochlorite of soda. Used with the same technique they sterilise wounds. Their action on the tissues has been studied on both sterile and infected wounds, by comparing the charts of sterilisation and the curves of cicatrisation. When used at a strength of less than 0.2 per cent., they do not interfere with the rapidity of repair. It has been observed sometimes, however, that an aqueous solution of 2 per cent. may produce lesions of the connective tissue which show themselves by diminution, and sometimes arrest, of cicatrisation.

With the collaboration of MM. Cohen, Daufresne, and Kenyon, Dakin investigated a certain number of substances of the same group, particularly the chloramines, in which the group $\mathrm{NCl}$ is separated from the benzene radicle by the group $\mathrm{SO}_{2} \mathrm{Na}$; the similar 


\section{THE PRINCIPLES OF THE TECHNIQUE 69}

naphthalene derivatives; the other similar dicyclic derivatives; the chloramines in which the group $\mathrm{NCl}$ is directly attached to the benzene radicle; the bromamines; and finally the products of the action of hypochlorites upon different proteid substances. He found that the substances which contain the group $\mathrm{NCl}$ also possess powerful bactericidal action. But the presence in their molecule of more than one $\mathrm{NCl}$ group does not increase their germicidal potency. Molecule for molecule, the germicidal action of many of these chloramines was greater than that of hypochlorite of soda. As to the substances derived from proteins under the influence of hypochlorite of soda, their antiseptic action was very powerful. But blood serum inhibited their potency, as it had done in the case of hypochlorite of soda and the aromatic chloramines.

While inquiring into the factors which control the germicidal action of chloramines, Dakin found that chloramines or bromamines destroy micro-organisms at a lower molecular concentration than the corresponding hypochlorites or hypobromites. They may not, therefore, be considered as the bio-chemical equivalents of these latter substances.

The germicidal action of chloramines is due to the fact that the substances such as proteins, amino-acids, urea, and ammoniacal salts, which constitute living organisms, contain nitrogen under a form which can attract the chlorine of the different species of chloramines. On the other hand, the chlorinating action of chloramines resembles that of the hypochlorites, but their antiseptic action is often much greater. This fact may be attributed, according to Dakin, either to a special 


\section{TREATMENT OF INFECTED WOUNDS}

obscure action of the chloramine molecule, or possibly to the elective chlorination of some constituent of the cells.

2. The Properties of Para-toluene-sodium-sulphochloramine. - Because of these many excellent qualities, para-toluene-sodium-sulphochloramine was chosen by Dakin for practical use in the sterilisation of wounds. This substance can readily be manufactured at a reasonable price by a method which has been described by Dakin. Para-toluene-sulphochlorate, a by-product in the manufacture of saccharine, is the basis. Several English houses are making it, and sell it under the name of "Chloramine T." Apart from its great germicidal potency, which has been noticed above, Chloramine $\mathrm{T}$ has other advantages. It does not coagulate proteid matters in the ordinary treatment of wounds. It is very soluble in water. That is an important factor. In short, chloramines, endowed with a high germicidal potency, very slightly soluble in water, but which could be dissolved in vaselin or lanolin, would be without practical value. Besides, Chloramine $T$ has the advantage over hypochlorite of being very stable. Dakin found that the decomposition of a solution kept in the dark for I 32 days, was inappreciable, whilst the solution exposed to daylight showed such a slight diminution of strength as to be scarcely noticeable. This stability of Chloramine $\mathrm{T}$ is a great advantage over Dakin's hypochlorite solution, which decomposes under the influence of light and heat.

Dakin's chloramine is employed in an aqueous I per cent. solution. As its stability is greater than that of hypochlorite, it has been employed by Daufresne in the 
THE PRINCIPLES OF THE TECHNIQUE $7 \mathrm{I}$

form of a paste, composed of stearate of soda mixed with varying proportions of chloramine. For general purposes a paste is used which contains I per cent. of chloramine and 8.6 per cent. of stearate of soda.

3. The Interaction of Chloramine and the Tissues.Chloramine, employed on the surface of a wound, in solutions of about I per cent., produces no retardation of cicatrisation. A large number of wounds have been treated with Daufresne's paste of sodium stearate, containing 4 to ro per cent. of chloramine, and the observed curve of cicatrisation always coincided with the curve calculated by du Nouy's formula. Fig. 28 shows the cicatrisation curves of a wound treated with a 10 per cent. paste of chloramine. Moreover, experiments made by Daufresne showed that a fragment of skin placed in a solution of chloramine retained its normal dimensions after the lapse of several days. The same is true of mortified tissues situated on the surface of wounds. In experiments conducted in vitro as well as in direct observations of wounds, it was found that chloramine does not dissolve the tissues. This is why it does not cleanse the surface of wounds as Dakin's solution does. It is not irritating, but it lacks one of the most necessary properties of an antiseptic, that of dissolving necrosed tissues. It should therefore be reserved for wounds whose surface is already cleansed, or for the skin and the mucous membranes.

While chloramine has little action on the tissues, the tissues, on the other hand, have a very marked action upon chloramine. Experiments were made with the peritoneum of a cat. When a 0.25 per cent. solution of chloramine is placed in a small cup containing a portion 
of the epiploön provided with its normal circulation, the rapid destruction of the antiseptic substance is observed. As a rule, the strength of a 0.25 per cent. solution of chloramine falls to 0.14 or 0.15 per cent. in about two minutes. Its decomposition is therefore much less rapid than that of hypochlorite. Chloramine will often lose only half its strength, while Dakin's hypochlorite loses more than four-fifths.

These experiments show that chloramine, despite its much greater stability, is rapidly decomposed when in contact with the tissues, and a considerable volume of solution must be employed to sterilise a wound.

In the sterilisation of a wound, the antiseptic plays a part comparable to that of the scalpel in a surgical operation. It is only an instrument, and does not constitute a method. But the choice of a good instrument is a factor indispensable to success. Chloramines and Dakin's hypochlorite are admirable instruments.

As Dakin's hypochlorite has the advantage of being strongly bactericidal, and only slightly irritating to the tissues, and at the same time can be readily manufactured at a cheap rate, it would seem that it ought to become the chosen antiseptic during this war.

\section{Contact of Antiseptic and Micro- ORGANISMS}

The antiseptic solution, only sterilising what it touches, must enter into intimate contact with the microbes infecting the wound. This contact has been considered impossible by the majority of modern surgeons. Sir Almroth Wright considers that, in the 
wounds inflicted in war, microbes are found so deeply buried in the irregularities of the wounds, in the middle of necrosed muscles and blood-clots, that it is hopeless to try to reach them by means of an antiseptic. It is also supposed that, in suppurating wounds, microorganisms inhabit the depths of granulation tissue, muscular interstices, and lymphatics, and that, in consequence, they are beyond the reach of substances poured over the surfaces of the wound. It is certain that if the topography of infection is such that the microbes cannot be brought into actual contact with the antiseptic, chemiotherapy of wounds ought to be abandoned.

But the opinion expressed by Sir Almroth Wright was based upon hypotheses and arguments, and not upon exact observation of what takes place in war-wounds. In order to find out if antiseptic treatment ought or ought not to be applied to infected wounds, it is necessary to study the topography of infection in both fresh wounds and suppurating wounds, and to inquire if it be possible to bring the antiseptic into contact with the microbes.

\section{A. Topography of Infection}

I. Fresh Wounds.-The topography of infection was studied at first in freshly inflicted wounds, superficial and deep, with fracture and without. Specimens of the secretions were taken from various regions of the wound, from around projectiles, shreds of clothing, splinters, and from the surface then examined by means of smears (p. 182) and cultures.

During the first few hours following the infliction 
of the wound, the smears in general showed no microbes, whilst cultures were positive. The apparent asepsis of the smears was due to two causes, the dilution by blood of the microbes infecting the wound, and their relatively small number at this early period of the infection. In fact, to show themselves in the secretions, the organisms need to have had the time to multiply and spread themselves from the foreign bodies on to the surfaces of the wound.

At the end of five or six hours, in wounds which are not bleeding, rods and cocci are sometimes found. These were localised in the regions close to the foreign bodies. Frequently also, no microbe was visible, though bouillon in which shreds of tissue taken from the immediate neighbourhood of the foreign bodies had been placed yielded abundant cultures, aërobic and anaërobic. The direct examination of foreign bodies, shell splinters, or particles of cloth, gave varying results. In general, no microbes were found on the surface of projectiles, although in more than half of the cases they gave positive cultures. Shreds of clothing, on the contrary, always yielded an abundant microbial flora. Often scrapings of fragments of great-coat, five or six hours after the infliction of the wound, showed some rod-like bodies; and nearly always anaërobic cultures made from these debris gave off gas abundantly.

At the end of about twelve hours, bacteriological examination practised under the same conditions, showed microbes more constantly and in greater abundance. ${ }^{1}$

I See also Policard et Phelip, $C$. $R$. de PAcadémie des Sciences, July 5, 1915. Fiessinger, La pratique de la chirurgie de guerre, 1916. Fiessinger et Montaz, C. R. Societt de Biologie, June 9, 1916. 


\section{THE PRINCIPLES OF THE TECHNIQUE 75}

Wounds commenced to react, and polynuclear cells appeared in numbers more or less great.

After twenty-four hours the topography of infection of the wound had greatly changed, for the bacterial harvest was no longer localised on the surface, or around foreign bodies. The examination of smears revealed the presence of microbes over almost the whole extent of the wound. At the same time a greatly increased number of polynuclear cells was to be seen. In short, during the first twenty-four hours there may be witnessed, first, the multiplication of microbes on the surface and in the neighbourhood of foreign bodies, especially of fragments of clothing, and later their diffusion over the superficies of the wound.

The modifications of the bacteriological aspect of a wound from the fifth or sixth hour to the twenty-fourth hour were due to the rapid division of micro-organisms. If it be supposed that each microbe divides every half-hour, it will give birth in twelve hours to more than fifteen million other microbes. This extreme rapidity of multiplication explains why wounds twenty-four hours old are already invaded by myriads of micro-organisms.

Close examination of a great number of wounds has shown that these micro-organisms remain, as a rule, on the surface of wounds, and do not penetrate deeply into muscular interstices nor into lymphatics. They invade blood-clots and tissues without circulation. They follow the blood poured out along vascular sheaths, and they may also bury themselves in fractured bones. But usually during the early hours, and even the first few days following the infection of the wound, they live on the surface of the tissues; that is to say, within 


\section{TREATMENT OF INFECTED WOUNDS}

reach of a liquid, if this liquid be applied under suitable conditions.

The existence of this fact has been made plain in the course of experiments made upon the wounds themselves. When the antiseptic liquid was brought into contact with the infected surface, the number of microbes rapidly diminished, and at the end of a short time the wound became completely aseptic. Wounds of the surface could be sterilised thus in twenty-four hours; irregular wounds, even when accompanied by fracture, became sterile in five or six days. The tissues were surgically sterile in their substance as well as on the surface. In fact, when the treatment had been applied from the beginning, it was possible to close up the wound by deep interstitial sutures, without this being followed by rise of temperature. Secondary operations practised in cases where wounds had been closed after sterilisation, did not determine the appearance of febrile phenomena. When comparing such results as these with what is observed always in the case of non-sterilised wounds, it is fair to conclude, with every semblance of probability, that the microbes have been destroyed in all parts of the wound.

Everything goes on as though during the first twentyfour hours, and sometimes during the early days following the receipt of the wound, the microbes dwelt on the surface of the wound ; consequently, within reach of the antiseptic. However, in irregular wounds and compound fractures microbes are sometimes found to be out of reach of the liquid. After some days of treatment the secretions of certain regions become aseptic, whilst those of other regions still remain infected. These 
regions have not been reached by the liquid, either because the latter has not been introduced deeply into the diverticula, or because the walls are protected against the antiseptic by sphacelated tissues, blood-clots, or a compress soaked in blood. Gauze compresses, bloodclots, or dead tissues have a peculiarly harmful effect, because they protect the bacteria from the attack of the antiseptic.

2. Suppurating Wounds.-During the period of suppuration, contact between the microbes and the antiseptic was, in general, more difficult to obtain. The number of microbes had greatly increased. No longer were there topographical differences in the volume of infection, for the bacteria were found in almost equal quantities in every part of the wound. But, following the shape and character of the wound, the microbes were reached by the liquid more or less easily. In surface wounds, and in irregular wounds of the soft parts, whose walls were covered with granulations and suppurated abundantly, the antiseptic rapidly destroyed the bacteria. But when the latter were protected by necrosed tissue, tendons, or aponeuroses which were being eliminated, the liquid could not reach them. and infection persisted. Even in long-standing wounds the contact between the liquid and the microbes was so complete that, in certain cases, the latter were seen to disappear completely in forty-eight hours.

The suppression of micro-organisms in secretions, and the possibilities of sterilising the surface of a wound in such a manner that suturing becomes possible, does not mean, however, that all the microbes have been brought into contact with the antiseptic and destroyed. In fact, 
when by means of sutures more or less deep wounds are brought together, which have suppurated for some time before being sterilised, there is sometimes re-infection and a rise of temperature. These phenomena are not seen in wounds which have been subjected to sterilisation from the beginning. But a wound which is cicatrising at the same time that it is suppurating, is keeping in its walls microbes capable of producing re-infection at the moment of a fresh traumatism. In wounds of long standing which are suppurating, antiseptics cannot reach microbes already enclosed in granulations, but they can affect those which are at the surface of the wound. As, on the other hand, living tissues destroy or encapsule microbes withdrawn from the antiseptic, sterilisation takes place little by little.

Therefore it is important to sterilise a wound at a period as near as possible to the onset of infection. If postponed to a later period, sterilisation is effected and closure by suture may be obtained; but microbes have already become enclosed in the cicatrix, and remain alive there. We have examined, on a wound more than six months old, a thick cicatrix which had formed during that long period of suppuration. The different layers of the cicatrix presented a varied bacterial flora. Passing from the deepest part to the surface, there was first a layer containing Welch's bacillus, next a sterile layer, then a stratum containing small rod-like bodies, lastly a layer of various cocci. In wounds of long standing, the topography of infection is therefore such that the antiseptic cannot reach the microbes in every part in which they are found. But, on the other hand, the microbes are enclosed or encapsuled in the tissues, and are not in 
a condition to work harm until a new traumatism sets them free.

From the practical point of view, in the suppurating wounds of soft parts, contact between microbes and antiseptic sufficient to assure surgical sterilisation is possible of attainment. In deep wounds with pus burrowing along muscular interstices, where contact between antiseptic and microbe cannot be realised, results are less favourable. When suppurating wounds are accompanied by fractures, or the osseous fissures described by Policard, ${ }^{1}$ along which the micro-organisms are propagated, it becomes impossible to make the liquid penetrate into all the infected places. Similarly, when osteo-myelitis has declared itself, or when splinters have been left in the tissues, the conditions are the same. Microbes establish themselves in the sequestra at such a depth that the antiseptic cannot penetrate to them. They are protected by their situation, at the same time against the chemical agent and against the polynuclear cells coming from normal tissues. This is the reason why the infection is so extremely tenacious, when bony lesions or necrosed splinters persist at the bottom of irregular wounds.

This brief examination of the topography of infection shows that in the majority of cases it is possible to obtain intimate contact between antiseptic and microbe. Suitable preparation of the wound for the penetration of the germicide substance, and distribution of this substance over the whole of the affected surface, will enable this contact to be realised. If, up to the present, we have not succeeded in chemically sterilising wounds, it is, in

${ }^{1}$ See also Bowlby, "Wounds in War," The Lancet, 1915, pp. 1388, 1389. 
part, because we have neglected to prepare them in such a manner that the antiseptic substance may reach every point where microbes exist.

B. Preparation of the Wound for the Penetration of the Antiseptic

Most important in the preparation of the wound is the mechanical cleansing of the infected regions. Free incisions in the soft parts allow this cleansing to take place even in the case of irregular torn wounds, accompanied by fracture. It is well known that shreds of clothing, projectiles, splinters lying free, blood-clots and necrosed tissues serve as shelters for microbes and protect them from the antiseptic. In consequence, every foreign body should be most carefully sought for and removed. Débris of clothing are the principal source of infection, and the antiseptic generally cannot penetrate them. Necrosed tissues are the favourite haunt of gas infection. Therefore they must be removed. Ever since the beginning of the war, Depage and the surgeons of his school have made a systematic resection of all tissues, skin, aponeuroses or muscles which were likely to mortify. This practice is excellent and ought to become general. All blood-clots are removed, and, to prevent their reoccurrence, careful hæmostasis of the whole of the wound is practised. The surface of bony cavities in which projectiles are lodged, is scraped, and resected if needful. Furthermore, it is well to remember that compresses placed in wounds efficiently protect microbes against antiseptics. Therefore a wound should never be left plugged with tampons or compresses. If an 


\section{THE PRINCIPLES OF THE TECHNIQUE 8r}

open wound is desired, tubes of large calibre perforated with many wide holes are used.

Incisions are made in such a manner that the diverticula of the wound are laid open as freely as possible. The liquid should penetrate everywhere, and remain in contact with the infected area as long as needful. As gravity plays an important part in the distribution of a liquid, those wounds which can be filled up like a cup are the most favourably qualified for sterilisation. That is the reason why wounds on the anterior surface of limbs are preferred to dependent counter-openings. Liquid is thus retained in the wound and its walls bathed more completely. Very large incisions need not be objected to, because they allow the topography of the wound to be studied and diverticula dealt with.

Once the wound has been thus freely laid open and all foreign bodies removed, the best possible conditions for contact between the liquid and the surfaces of the wound are obtained. It only remains to make arrangements for the application of the antiseptic to the whole of the infected surface.

\section{Application of the Antiseptic}

It is indispensable to place the liquid in direct contact with the tissues in the deepest regions of the wound. Distribution of a liquid over the whole extent of an irregular surface is difficult to accomplish.

The simplest method, which at once occurs to every one, is to use absorbent gauze or other fabric, or strands of cotton-wick, conducting by capillary action the liquid from an external reservoir over the whole surface of the 
wound. This arrangement has been adopted by Sir Almroth Wright in his dressings of hypertonic saline solution. At the outset of our researches on the sterilisation of wounds we employed a similar method. Layers of absorbent tissue were applied to the surface of the wound, a rubber tube led the liquid to the tissue to which was entrusted equal distribution to all parts of the wound. Experience was not slow in making clear to us that procedures based on this principle were incapable of producing efficient contact of the antiseptic with the surfaces of the wound. In fact, at the end of a few hours the deepest part of the conducting tissue became impregnated with plasma or pus, and impermeable to the antiseptic liquid. On casual examination, the apparatus appeared to be working well, but the liquid went into the tissue without moistening the raw surfaces. This method of conducting the liquid was abandoned entirely. It has only been retained to distribute liquid on the surface of a rubber tube pierced with small holes. These tubes covered with tissue are sometimes used during the first few hours following the infliction of a wound, because at this period secretion is slight. In all other cases we use absorbent tissue compresses, which by a special arrangement cause the liquid to flow between themselves and the wound.

The procedure which has been adopted consists in distributing the liquid to all parts of the wound by means of rubber tubes, utilising the force of gravity of the liquid. The disposal of these tubes varies with the shape and situation of the wound. In wounds which have only a single opening so situated that they can be filled up like a cup, permanent contact between the 


\section{THE PRINCIPLES OF THE TECHNIQUE 83}

antiseptic and the surfaces is assured by introducing a rubber tube to the bottom of the cavity (Fig. 3I). If the patient reclines in a suitable position, the wound remains full of antiseptic liquid. But in dealing with surface wounds (Fig. 32)-large, irregular wounds, and those with several wide openings (Fig. 33)--it becomes more difficult to distribute the liquid over the whole surface. The most practical method consists in allowing small

FIG. 3r.-Wound with superior opening which can be filled like a cup.

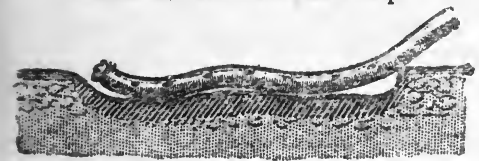

FIG. 32. - Surface wound receiving liquid from a tube perforated by small holes.

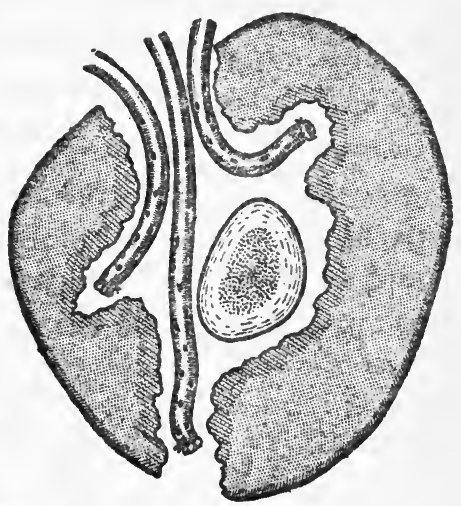

FIG. 33.-Irregular wound with several perforated tubes in its diverticula.

rubber tubes perforated with minute holes to lie on the tissues. The holes number fifty to each tube, and have a diameter of about 0.5 millimetre. When these tubes are charged with liquid under pressure, the surface of the wound is moistened by the fluid which issues from all the orifices. This procedure has been adopted, in the first place, because it is successful, and next, because it can be carried out by means of articles readily 


\section{TREATMENT OF INFECTED WOUNDS}

obtainable commercially. The tubes should be tied up at one end and the perforations made with an ordinary punch.

But this manner of distribution is far from ideal, because, the holes being too large and not sufficiently numerous, the liquid spurts out too profusely over a space too limited. So it is not made the best use of. Probably a tiny hose, pierced with a great number of microscopic holes, or rather rubber membranes, whence the antiseptic could ooze out, would bring about more intimate relations between liquid and microbes. A totally different arrangement might be conceived, by which the liquid could be distributed over the surface of the wound without using tubes at all. If the antiseptic were incorporated with a substance which had the property of melting very slowly in contact with the tissues, and which at the same time could be moulded to fit all the irregularities of the wound, a more perfect distribution of the antiseptic would be attained.

\section{Maintaining the Concentration of the ANTISEPTIC}

The second essential principle is the keeping the fluid on the surface of the tissues at a constant degree of concentration. $U p$ to the present, this principle has been completely ignored. As a rule, antiseptics are applied to wounds by means of absorbent gauze, and the liquid renewed once or twice in the twenty-four hours. It is certain, however, that, under these conditions, the bactericidal power of the substances employed rapidly vanishes. In fact, if a compress soaked in Dakin's 
solution 0.5 per cent. be applied to the surface of a wound, the result obtained is almost nil, because the concentration of the solution lessens very quickly, under the influence of dilution by the secretions of the wound, and the combination of hypochlorite of soda with the proteins of pus, of the tissues, and of blood. In a word, the degree of concentration of an antiseptic applied according to the usual surgical method at once becomes so feeble that no result can be hoped for. The only way to maintain at the needful strength, on the surface of a wound, a solution which is constantly being diluted and destroyed, is to keep on renewing it, unceasingly. For this reason we have used instillation, continuous or intermittent. The best method consists in allowing a current of the antiseptic liquid to flow very slowly over the whole surface of the wound. In the case of small wounds, and of those which can be filled with liquid like a cup, this is readily done. The antiseptic, supplied drop by drop, is slowly renewed, in contact with the tissues. When it escapes from the wound, it is absorbed by the dressing, and evaporates without wetting the patient. But when the wound is of large extent, and presents several openings, a considerable quantity of liquid would be needed to keep the whole raw surface continually moist. The amount which would escape from the wound would be too great to be absorbed by the dressing. One has to revert to the old process of continuous irrigation, which is complicated, and distressing to the patient.

On the other hand, experience has shown that if the liquid be applied over the surface of the wound every hour or every two hours, sterilisation is attained. This 
intermittent instillation is easy to apply. It is the procedure we are at present employing. It is far from being perfect, but it allows of the frequent contact of the surfaces of the wound with the antiseptic at a known degree of concentration. Better arrangements for keeping up both supply and strength of the antiseptic will doubtless be found. For example, if the liquid were to issue from numerous microscopic apertures in tubes inserted in all the cavities of the wound, the quantity needed would be smaller, and yet every part of the wound would be bathed incessantly by the antiseptic at the desired strength.

The degree of concentration of the antiseptic has been determined empirically. It is found that Dakin's solution, containing 0.45 to 0.5 per cent. of hypochlorite of soda, applied under the conditions just described, does no harm to tissues and sterilises wounds.

\section{Duration of the Application of the ANTISEPTIC}

An essential point of the method is the prolonged application of the antiseptic. This principle seems to have been neglected as much as the preceding. Although experiments in vitro have shown that microbes, to be destroyed, must be immersed in the antiseptic solution for some considerable time, yet people persisted in believing that, under the much more unfavourable conditions of the clinic, sterilisation of a wound could be obtained by brief contact between bactericidal substance and microbes. That is why so many surgeons still remain loyal to the rite of washing over a wound with an antiseptic liquid. 
They imagine that if a liquid has flowed over the surface of a wound for four or five minutes, often much less, that wound will become sterile. It is certain, however, that to obtain any action the antiseptic must remain on the wound for a much longer period.

A. Experiments showing the Necessity for Prolonged Contact.-In the following clinical experiments it was sought to discover what should be the length of time for the application of the hypochlorite.

In the first place, the influence of hypochlorite applied as is usual in a wet dressing was examined. Upon surface wounds, whose bacteriological condition was known, compresses were placed soaked in a 0.5 per cent. solution of hypochlorite. The next day the number of microbes had not undergone any appreciable change. Gauze "wicks" soaked in hypochlorite were also introduced into deep wounds. At the end of twenty-four hours, the surface of the compresses yielded a large number of microbes.

The insufficiency of the technique usually employed was thus demonstrated. Next, the length of time during which hypochlorite was present in the wounds was lengthened by soaking the dressing with the antiseptic three times a day. In most cases the "smears" showed a marked lessening in the number of microbes; but the wounds more deeply infected showed no change. On one small wound dressed three times a day with hypochlorite, the number of microbes did not diminish. At the end of a week of this useless treatment, a small quantity of hypochlorite was injected hourly over the surface of the wound, under the compresses. All the microbes disappeared (Fig. 34). 
A large number of similar experiments showed that, in surface wounds, the infection did not resist instillations of hypochlorite every two hours during one or two days. In deep wounds, diminution in the number of microbes came about more slowly, even under the influence of frequent instillations of hypochlorite. Daily examination of the "smears," made from the discharges

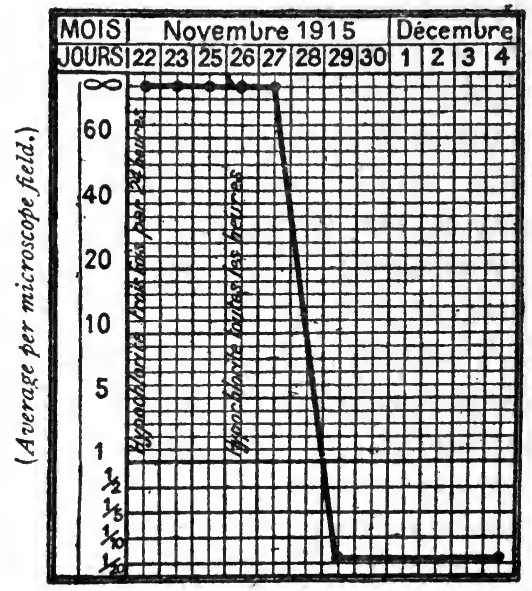

FIG. 34.-Necessity for prolonged contact between the antiseptic and the wound. Highly infected atonic wound treated up to Nov. 26th by applications of hypochlorite three times a day. No diminution in the number of microbes. Nov. 26th, Dakin's hypochlorite was applied every hour. Sterilisation was attained Nov. 29th. ( $\infty$ denotes infinity.-Trans.)

from different regions of wounds of the soft parts, more or less irregular, showed that microbes often took four or five days to disappear. In severe lacerations of the soft parts, or in compound fractures, the application of the hypochlorite had to be continued usually eight, ten, or fifteen days before sterilisation was achieved.

In wounds complicated by splintered fractures 
it was sometimes impossible to get complete sterilisation. Generally the persistence of infection was due to the presence of a foreign body, projectile, splinter, or shred of clothing. When the foreign body was removed, sterilisation came about. Disinfection of deep wounds always takes longer than that of surface wounds. With the technique now in use, compound fractures are sometimes disinfected in five or six days. As a general rule, sterilisation requires ten, fifteen, or twenty days, or even more, if it is a question of a compound fracture of the thigh.

B. Relation between the Dimensions of a Wound and the Time required for Sterilisation.-It will be well to inquire why the duration of application of the antiseptic has to be longer for compound fractures with large wounds, than for surface wounds.

We have often seen surface wounds yielding many microbes become sterilised in forty-eight hours. The tardiness of sterilisation in irregular wounds appears to be due to the presence of diverticula into which the liquid does not penetrate, and where microbes swarm, or to the presence of sphacelated tissues which shield the microbes from the attack of the antiseptic. However, in surface wounds, with sphacelated tissue, sterilisation is brought about more quickly than in large and irregular wounds. Therefore it is probable that imperfection of technique alone renders necessary a prolonged application of the antiseptic. There is not, in fact, any theoretic reason why a large and irregular wound should sterilise more slowly than a small wound with even walls. But it is much more difficult to make the liquid penetrate all the irregularities of a deep wound than it is to bring it into contact with the entire surface of a smooth one. 
The duration of the application of hypochlorite in deep wounds will lessen when it becomes possible to apply the antiseptic continuously to the entire surface of the wound. Our technique is still too clumsy, and the methods of distributing the liquid in use to-day do not succeed in placing every portion of a large wound simultaneously under the influence of the bactericidal substance. It is quite probable that different portions of an extensive wound are sterilised successively, for the bacteriological examination shows that after the lapse of several days certain regions of a wound are sterile, whilst others still continue to harbour microbes. Besides, rapidity of sterilisation increases to a certain extent with the quantity of liquid employed, that is to say, it depends on the extent of the surface of the wound which is acted on by the liquid. It is permissible to believe that improvements in technique will lessen the period during which antiseptic instillations will need to be employed, but it is unlikely that this period will be cut down to less than twenty-four hours.

\section{KNOWLEDGE OF THE BACTERIOLOGICAL CONDITIONS OF THE WOUND}

The bactericidal potency of the chloramines and of hypochlorite is such that every wound should respond to the treatment by a diminution in the number of microbes and by their final disappearance. Therefore it is important to ascertain if the bacteriological condition is being modified in a progressive manner. In fact, when that condition remains stationary, it may be concluded that contact between antiseptic and microbes is 
THE PRINCIPLES OF THE TECHNIQUE 9I not completely established, and that the technique needs alteration.

Clinical observation alone does not enable us to follow the evolution of wound infection. It gives only the probabilities. When a patient has ceased to have pyrexia, when the wound is of a healthy red, when its margins are supple and when suppuration has disappeared, then it is fair to assume that the wound is nearly aseptic. But investigation has taught us that wounds looked upon as aseptic are often highly infected, and that it is never safe to trust the favourable appearance of the tissues as evidence that they are sterile. Besides, it often happens that wounds treated by chloramines have a slightly greyish look, and are covered with purulent secretion. They have the appearance of infected wounds. However, these wounds may be sutured without the least rise of temperature following. In this case only bacteriological examination can demonstrate to the surgeon that the pus which covers the granulations is aseptic.

It is impossible, therefore, to ascertain the results of treatment with sufficient precision, without the constant aid of the microscope. Using the simple method which will be described later, bacteriological examination of a large number of wounds may be made every day. M. Gaultier ${ }^{1}$ has shown that, even in dressing-stations (ambulances, Fr.) at the front, it is possible to make use of the microscope. That examination gives warning of the existence of errors of technique as soon as they appear, and so enables the loss of time to be avoided which is the usual consequence. It points out the 1 Gaultier, Paris Médical, July, rgr6. 
92 TREATMENT OF INFECTED WOUNDS

moment when the wound has become surgically sterile and can be sutured.

To sum up, knowledge of the bacteriological condition of the wound is an indispensable part of the technique of sterilisation, and it alone can give to the latter the necessary precision. 


\section{CHAPTER II}

THE TECHNIQUE OF THE MANUFACTURE OF DAKIN'S SOLUTION

HYPOCHLORITE of soda was discovered by Berthollet in I788, and its antiseptic properties have been known for a long time. Labarraque gained great renown by embalming, by the aid of his liquor, the corpse of Louis XVIII., which was so extremely decomposed that no one could come near it. But neither Labarraque's solution nor eau de Javel can be used with safety in surgery. One of the essential conditions of the sterilisation of wounds is s as is well known, the employment of a substance which, in a given degree of concentration, can be applied for a long period to wounds without irritating them. This is the reason why it is impossible to use commercial hypochlorites, whose content of hypochlorite is extremely variable and which contain free alkali. The proportion of alkali contained in eau de Javel and Labarraque's liquor is great enough to produce solution of the skin, if the contact be sufficiently prolonged.

\section{DAkin's Technique}

That is why Dakin sought the means of obtaining a solution deprived of free caustic alkali, and whose content of hypochlorite must not vary beyond the limits 


\section{TREATMENT OF INFECTED WOUNDS}

of 0.45 and 0.50 per cent. Later experiments by Daufresne showed that below 0.45 per cent. the solution is insufficiently active, whilst above 0.5 per cent. it is irritating. At the time of his communication to the Académie des Sciences, Dakin gave a method of preparation of this solution which enabled it to be made with the simplest appliances, without chemical knowledge.

"I40 grammes of anhydrous carbonate of soda, or 400 grammes of the crystallised salt, are dissolved in Io litres of ordinary water, and 200 grammes of chloride of lime ${ }^{1}$ of good quality are added to it. The mixture is well shaken, and at the end of half an hour the clear liquid is siphoned off and filtered through cotton. To the filtrate are added 40 grammes of boric acid, and the solution thus obtained may be used at once; it does not colour phtalein in suspension in water." 2

This very simple mode of preparation was easy to execute, a great advantage for hospitals at the front. But experience in its use has brought to light several inconveniences, which have been studied by Daufresne. One of the products used in its preparation, chloride of lime, being of very variable composition, its content of active chlorine might vary from the normal to double the amount. Under the influence of humidity it forms compact masses, which, when agitated with the solution of carbonate of soda, are incompletely broken up, and only yield a portion of their hypochlorite. These are the reasons why defective solutions have sometimes been obtained while following conscientiously the procedure just described.

1 The salt referred to is of course not calcium chloride, but calcium chlorate, or chlorinated lime, universally known as chloride of lime (Tr.)

${ }^{2}$ Dakin, Presse Médicale, loc. cit. 
On the other hand, Daufresne was led to attribute certain irritation phenomena to the boric acid employed to neutralise the solution. In fact, without being able to give an exact account of the chemical reactions which enter into the change, every time the quantity of boric acid used to arrive at non-coloration in the phtalein test exceeds 4 grammes per litre, the solution becomes unstable and painful.

Besides, the solutions of hypochlorite prepared with boric acid, even in correct quantity, keep badly.

II. Dakin's Solution PREPARED BY DAUfRESNE'S METHOD

Having repeatedly examined similar solutions, Daufresne sought a remedy for these inconveniences by a more accurate mode of preparation which would give constant results. After numerous trials he decided on the following process, which necessitates the employment of three products, all easily procured :

Chloride of lime,

Carbonate of soda, anhydrous (Solvay),

Bicarbonate of soda.

A. Chloride of Lime and its Titration.-I. The chloride of lime of commerce is obtained by the action of gaseous chlorine on powdered slaked lime. It presents great variations in composition; notably in its content of active chlorine. Its chemical constitution, in spite of the numerous discussions of which it has been the subject, is not yet established in any satisfactory manner. Whatever it may be, we know that under the solvent action of water it yields three substances, hypochlorite and chloride of 
calcium and small quantities of lime, the residue being made up of excess of lime, partially carbonated.

It is an ordinary thing to meet with samples which differ greatly in richness. We have had occasion to examine a small number of lots whose titrage in active chlorine varied from $20^{\circ} 45$ per cent. to $35^{\circ} 90$. It will, therefore, be of advantage to use only a chloride of lime of known titration, so that we may employ a quantity correctly calculated according to its titration.

2. Titration of Chloride of Lime.-The estimation of this quantity is made in the following manner by Daufresne: Weigh out an average sample of 20 grammes, stir it up in a litre of water as thoroughly as possible, and allow it to stand some hours. Measure off Io c.c. of the clear liquid, add to it 20 c.c. of a 10 per cent. solution of iodide of potassium, 2 c.c. of acetic acid or hydrochloric acid, then to the mixture add drop by drop a decinormal solution of hyposulphite of soda $(2.48$ per cent.), up to decoloration. The number $n$ of c.c. of hyposulphite employed, multiplied by 1,775 , will give the weight $\mathrm{N}$ of active chlorine contained in 100 grammes of the chloride of lime.

This estimation must be carried out for each consignment received. The following table drawn up by Daufresne gives directly, according to the amount of active chlorine contained in the chloride of lime, the quantities of the reagents required to obtain automatically a correct solution :- 


\section{PREPARATION OF DAKIN'S SOLUTION 97}

\begin{tabular}{|c|c|c|c|}
\hline \multirow{2}{*}{$\begin{array}{c}\text { Titration of chloride of lime } \\
\text { ( } \mathrm{Cl} \%) \text {. } \\
\text { (English degrees.) }\end{array}$} & \multicolumn{3}{|c|}{$\begin{array}{l}\text { Quantities to be used to obtain xo litres of solution of } \\
\text { hypochlorite of } 0^{\circ} 475 \% \text {. }\end{array}$} \\
\hline & $\begin{array}{l}\text { Chloride of } \\
\text { lime. }\end{array}$ & $\begin{array}{c}\text { Carbonate of } \\
\text { soda, anhydrous. }\end{array}$ & $\begin{array}{l}\text { Bicarbonate of } \\
\text { soda. }\end{array}$ \\
\hline $\begin{array}{l}20 \\
21 \\
22 \\
23 \\
24 \\
25 \\
26 \\
27 \\
28 \\
29 \\
30 \\
31 \\
32 \\
33 \\
34 \\
35 \\
36 \\
37\end{array}$ & $\begin{array}{c}\text { grms. } \\
230 \\
220 \\
210 \\
200 \\
192 \\
184 \\
177 \\
170 \\
164 \\
159 \\
154 \\
148 \\
144 \\
140 \\
135 \\
132 \\
128 \\
124\end{array}$ & $\begin{array}{r}\text { grms. } \\
\text { I I } 5 \\
\text { I I0 } \\
105 \\
100 \\
96 \\
92 \\
89 \\
85 \\
82 \\
80 \\
77 \\
74 \\
72 \\
70 \\
68 \\
66 \\
64 \\
62\end{array}$ & $\begin{array}{c}\text { grms. } \\
96 \\
92 \\
88 \\
84 \\
80 \\
76 \\
72 \\
70 \\
68 \\
66 \\
64 \\
62 \\
60 \\
59 \\
57 \\
55 \\
53 \\
52\end{array}$ \\
\hline
\end{tabular}

The determination of the quantity of soluble calcium in the chloride of lime would have a certain importance if one were not obliged, in order to obtain a product having some degree of stability, to use an amount of carbonate of soda far above that theoretically indicated. In fact, a solution prepared by the interaction of chloride of lime and salts of soda in theoretic quantities loses the whole of its hypochlorite in from 15 to 20 days.

B. Salts of Soda.-It is more convenient to use dry carbonate of soda (carbonate de soude, Solvay, Fr.) which is to be preferred to the other commercial salts because of its being anhydrous, pulverulent, and free from caustic alkali.

When obliged to use the hydrated salt (crystals), the 


\section{TREATMENT OF INFECTED WOUNDS}

quantity needful will be 285 grammes for Ioo of the dry salt.

Bicarbonate of soda is readily obtainable. It is always anhydrous. The solution should be made in the cold, because it commences to break up towards $50^{\circ} \mathrm{C}$.

C. Preparation of Dakin's Solution by Daufresne's Method.-I. To prepare Io litres of solution weigh out exactly the quantities of chloride of lime, carbonate of soda, and bicarbonate of soda determined when titrating the chloride of lime;

2. Place in a I2-litre flask the chloride of lime with 5 litres of tap-water; shake vigorously two or three times and leave it overnight;

3. Dissolve the carbonate and bicarbonate of soda in 5 litres of cold water.

4. Pour the solution of soda salts rapidly into the flask containing the chloride of lime, shake vigorously for about a minute, and put it aside for the carbonate of lime to settle ;

5. After the lapse of about half an hour siphon the clear liquid and filter it through a double filter-paper, in order to obtain a perfectly clear product, which must be kept cool, and protected from the light.

An excellent means of stabilising hypochlorite of soda consists in adding I : 200,000 of permanganate of potash ( 5 milligrammes per litre of filtered solution).

The antiseptic solution is then ready for surgical use. It would contain 0.35 to 0.50 per 100 of hypochlorite of soda, with small quantities of neutral soda salts. It is practically isotonic with blood-serum. ${ }^{1}$

1 The freezing-point of these solutions is very slightly higher than that of blood-serum. $\Delta=-0.60^{\circ}$ to $-0.65^{\circ}$ Cent. 


\section{PREPARATION OF DAKIN'S SOLUTION 99}

The action of water upon the chloride of lime not being inslantaneous, the product often contains lumps, from which the hypochlorite does not readily diffuse. The following experiment of Daufresne's is extremely instructive in this connection :

Io grammes of chloride of lime giving on titration 28.25 per cent. of active chlorine are placed in a flask with 1000 c.c. of distilled water. The whole is shaken vigorously for two minutes, and the solution titrated. Similar titrations are made from time to time.

\begin{tabular}{l|l|l|l|l|l}
\hline $\begin{array}{c}\text { Original titration of. } \\
\text { the solution (in. Cl \%). }\end{array}$ & t hour. & I hour. & 2 hours. & 6 hours. & 12 hours. \\
\hline 0.089 & 0.176 & 0.206 & 0.259 & 0.281 & 0.282 \\
\hline
\end{tabular}

As will be seen, solution is complete only after some hours' maceration; for which reason we have prescribed, in our technical directions, a prolonged contact between the chloride of lime and the water.

When the solution of carbonate and bicarbonate of soda is poured into the maceration of chloride of lime, an abundant precipitate of carbonate of lime appears, the result of the double decomposition which takes place between the soluble constituents of the chloride of lime and the soda salts.

The two principal reactions are:-

$$
\begin{aligned}
& (\mathrm{ClO})_{2} \mathrm{Ca}+\mathrm{CO}_{3} \mathrm{Na}_{2}=\mathrm{CO}_{3} \mathrm{Ca}+2 \mathrm{ClONa} \\
& \mathrm{CaCl}_{2}+\mathrm{CO}_{3} \mathrm{Na}_{2}=\mathrm{CO}_{3} \mathrm{Ca}+2 \mathrm{NaCl}
\end{aligned}
$$

but the chloride of lime always contains a residue of nonchlorinated lime, which may amount to 20 per cent. of 


\section{IOO TREATMENT OF INFECTED WOUNDS}

the total weight, and of which a small quantity dissolves in water during the course of preparation. This lime in its turn intervenes in a secondary reaction, when the formula is only concerned with the carbonate of soda :

$$
\mathrm{Ca}(\mathrm{OH})_{2}+\mathrm{CO}_{3} \mathrm{Na}_{2}=\mathrm{CO}_{3} \mathrm{Ca}+2 \mathrm{NaOH}
$$

setting free a small quantity of alkali, to which the classic Labarraque's liquor owes its causticity. In Dakin's process, this alkali is neutralised by an excess of boric acid.

In Daufresne's process no caustic soda is formed; the liquor contains, in fact, a certain quantity of carbonic acid, feebly combined (that of the bicarbonate of soda), which attaches itself to the lime as soon as the two solutions come into contact.

It is difficult to demonstrate with certainty what is the intimate mechanism of fixation of the lime, but it may be imagined. As a matter of fact, it is for the carbonic acid, amongst all the substances present, that lime possesses the greatest affinity. Henceforward, the harmful part played by the lime is suppressed, and the secondary reaction we have indicated is changed into one perfectly inoffensive :

$$
\mathrm{Ca}(\mathrm{OH})_{2}+2 \mathrm{CO}_{3} \mathrm{NaH}=\mathrm{CO}_{3} \mathrm{Ca}+\mathrm{CO}_{3} \mathrm{Na}_{2}+2 \mathrm{H}_{2} \mathrm{O}
$$

D. Titration of the Solution of Hypochlorite. ${ }^{1-M e a s u r e}$ Io c.c. of the solution, add 20 c.c. of ten per cent. solution of iodide of potassium, 2 c.c. of acetic acid, then drop by drop a decinormal solution of hyposulphite up to decoloration. The number of c.c. used, multiplied by 0.03725 , will give the weight of hypochlorite of soda contained in 100 c.c. of solution. 


\section{PREPARATION OF DAKIN'S SOLUTION IOI}

In the first stage of the determination, hypochlorite displaces the iodine of the iodide of potassium according to the equation

$$
\mathrm{ClONa}+2 \mathrm{KI}+\mathrm{H}_{2} \mathrm{O}=\mathrm{I}_{2}+2 \mathrm{KOH}+\mathrm{NaCl}
$$

which is only complete in the presence of a quantity of acid sufficient to saturate completely the liberated potash. The operation returns finally to an estimation of iodine by hyposulphite of soda :

$$
\mathrm{I}_{2}+2 \mathrm{~S}_{2} \mathrm{O}_{3} \mathrm{Na}_{2}=2 \mathrm{NaI}+\mathrm{S}_{4} \mathrm{O}_{6} \mathrm{Na}_{2}
$$

Examining the various reactions, we see that a single molecule of hypochlorite decomposes two molecules of iodide of potassium with liberation of two atoms of iodine, and that each atom of iodine transforms a molecule of hyposulphite into tetra-thionate of soda; thus :

$$
\begin{gathered}
\text { I mol. } \mathrm{S}_{2} \mathrm{O}_{3} \mathrm{Na} \rightarrow \text { I atom of } \mathrm{I} \rightarrow \frac{1}{2} \text { mol. } \mathrm{ClONa} \\
248
\end{gathered}
$$

On the contrary, if as in the estimation of chloride of lime the result had to be determined in active chlorine (decolorising chlorine), it would have been necessary to take into account that one atom of chlorine only displaces one atom of iodine:

$$
\begin{aligned}
& 2 \mathrm{Cl}+2 \mathrm{KI}=2 \mathrm{I}+2 \mathrm{KCl} \text { and } \\
& \qquad 2 \mathrm{I}+2 \mathrm{~S}_{2} \mathrm{O}_{3} \mathrm{Na}_{2}=2 \mathrm{NaI}+\mathrm{S}_{4} \mathrm{O}_{6} \mathrm{Na}_{2}
\end{aligned}
$$

and

I mol. $\mathrm{S}_{2} \mathrm{O}_{3} \mathrm{Na}_{2} \rightarrow$ I atom of $\mathrm{I} \rightarrow$ I atom of $\mathrm{Cl}$ 248

35.5

The equations (in the case of a sample of ro c.c.) which give the activity of a solution of hypochlorite, will be different, according as the result is expressed, either 
directly in hypochlorite, or indirectly in the quantity of chlorine of equivalent activity.

Hypochlorite per cent.

Active chlorine per cent.
$\mathrm{N} \times 0.03725$

$\mathrm{N} \times 0.0355$

It is necessary to insist on this point, because the same coefficient of activity is sometimes wrongly attributed to the hypochlorite as to the chlorine.

E. Electrolytic Hypochlorite.-Electrolytic hypochlorite of soda is neutral. It contains no foreign substance excepting an excess of chloride of sodium. A 0.5 per cent. solution of this hypochlorite possesses the same bactericidal power as Dakin's chemically prepared solution. Its solvent action on mortified tissues is less rapid, but it has the advantage of being less irritant to the skin.

Electrolytic hypochlorite has not been employed hitherto because it is unstable. In a few days, sometimes in a few hours in warm climates, the solution decomposes. We began to employ electrolytic hypochlorite of soda from the time when Daufresne found a way of stabilising it. Daufresne discovered that if an addition of I part in 20,000 of permanganate of potash, or 0.2 per cent. of a soda salt, such as silicate of soda, be made to the electrolytic solution, it keeps sufficiently well. We may therefore employ the electrolytic hypochlorite to-day in all cases where the special sensitiveness of the skin or the length of treatment makes it desirable to use a solution less irritating than Dakin's chemically prepared solution. 


\section{Keeping Qualities of the Solution}

Solutions of hypochlorite do not keep indefinitely, they change very slowly in the dark, much more quickly in the light. Daufresne studied the influence of light in the following manner.

Portions of the same solution of known strength being placed in two flasks, one flask was left on the laboratory table exposed to diffused light, while the other was kept in a cupboard. He ascertained that the activity of the solution sheltered from the light had not sensibly varied, whilst the first had lost about 20 per cent. of its hypochlorite.

\begin{tabular}{l|l|l|l|l|l|l|l}
\hline & 0 day. & 7 days. & I5 days. & 2I days. & 30 days. & $\begin{array}{c}\text { Loss in } \\
\text { one } \\
\text { month. }\end{array}$ \\
\hline $\begin{array}{c}\text { Solution kept in the } \\
\text { light, titration. }\end{array}$ & 0.505 & 0.497 & 0.452 & 0.41 I & 0.380 & $24 \% \%$ \\
$\begin{array}{c}\text { Solution kept in the } \\
\text { dark, titration. }\end{array}$ & 0.505 & 0.505 & 0.502 & 0.500 & 0.497 & $\mathbf{1 . 4} \%$ \\
\hline
\end{tabular}

When the mass of liquid is considerable, the alteration is extremely slow. Daufresne kept a solution of hypochlorite of 0.502 per cent. of $\mathrm{ClONa}$ in a wickercovered carboy of black glass, 25 litres, without any special precautions as regards light. At the end of $3 \frac{1}{2}$ months the titration gave $-0^{\circ} 493$ per cent. of $\mathrm{ClONa}$, a loss practically negligible.

What becomes of the hypochlorite? One cannot say with certainty. By analogy with what happens, under the influence of heat, it is thought that the hypochlorite tends towards its two stable forms, chloride and chlorate of sodium :

$$
{ }_{4} \mathrm{ClONa}=3 \mathrm{NaCl}+\mathrm{ClO}_{3} \mathrm{Na}+\mathrm{O}
$$




\section{IO4 TREATMENT OF INFECTED WOUNDS}

Obviously, this reaction implies a release of oxygen, which is sometimes lacking. Besides, it does not explain all the facts observed. It is sufficient to remember that, in practice, it is better to keep the solutions away from the light, and still more important to renew them as frequently as possible, every ten or fifteen days at least.

Like the chemical hypochlorite, the electrolytic hypochlorite must be kept away from the light. Even under these conditions it rapidly degenerates when it is pure. But the addition of permanganate of potash or an alkaline salt greatly improves its keeping qualities. The following table gives the results of a series of tests carried on over a period of three weeks :-

I. Electrolytic hypochlorite kept in the) light . . . . . . .

2. Electrolytic hypoclorite kept in darkness

3. Electrolytic hypochlorite with the addition of $1: 20,000$ of permanganate of potash, kept in the light

4. Electrolytic hypochlorite with the addition of $I: 20,000$ of permanganate of potash, kept in darkness

5. Electrolytic hypochlorite with the addition of 0.2 per cent. of silicate of soda, kept in the light . . . .

6. Electrolytic hypochlorite with the addition of 0.2 per cent. of silicate of soda, kept in darkness

\begin{tabular}{|l|l|l}
\multicolumn{2}{|c|}{ Titration in ClONa \% } \\
on the
\end{tabular}

Inspection of this table shows that electrolytic hypochlorite can be so far stabilised as to undergo no alteration of importance for a period of two or three weeks. 
IV. COMPARISON OF DAKIN'S SOlution WiTH LABARRAQUE'S LiquOR AND COMMERCIAL EAU DE JAVEL

The mistake is often made of identifying Labarraque's liquor and even commercial eau de Javel with Dakin's solution. But Daufresne has shown by simple methods, that, from the biological as well as the chemical point of view, these three solutions behave in very different ways.

Amongst the reactions which may be cited for this purpose, two are particularly characteristic. The phenolphtalein reaction and the effect upon skin. In these experiments the three solutions are brought to a convenient strength of ( $\mathrm{Ogr} .50 \%, \mathrm{Fr}$ ) of hypochlorite of soda. The action upon skin has already been described (p. 23). We shall here give only the action upon phenol-phtalein.

If 20 c.c. of the solution to be examined are poured into a beaker and on the surface are placed a few centigrammes of phenol-phtalein in powder, it is seen that :-

Ist. Eau de Javel and Labarraque's liquor immediately colour the particles of phenol-phtalein an intense red, and the slightest shaking will suffice to communicate to the whole of the liquid a bright red colour, which slowly disappears under the decolorising action of the hypochlorite.

2nd. Dakin's solution, under the same conditions, does not give any colour to the particles of phtalein, and it is only after vigorous and prolonged shaking that the liquid becomes of a faint rose tint. 


\section{IO6 TREATMENT OF INFECTED WOUNDS}

Then, if one seeks the amount of alkalinity which a solution must possess in order to give so much colour to powdered phtalein, it is found that only solutions containing at least 0.2 per cent. of caustic alkali will give to the phtalein test a similar degree of colour. Carbonate of soda gives only an almost imperceptible tinge to the particles of phtalein, and a rosy tint to the liquid: that same solution gives no colour if it only contain $2{ }^{\prime} \mathrm{O}$ per cent. carbonate of soda.

Therefore Labarraque's liquor and eau de Javel each contain a small quantity of caustic soda, revealed by the phenol-phtalein test, and which might readily be foreseen after examination of their mode of preparation.

In fact, Labarraque's liquor and many samples of commercial eau de Javel are obtained by double decomposition of a solution of chloride of lime and a solution of carbonate of soda. All the constituents of chloride of lime (hypochlorite of calcium, chloride of calcium, slaked lime) are able to react upon carbonate of soda, giving respectively hypochlorite of soda, chloride of sodium and caustic soda. This caustic alkali, which constitutes the irritating element most to be dreaded in hypochlorite solution, certainly exists in the earlier stages of preparation as given by Dakin; but later it is neutralised by excess of boric acid. We have seen why it is not formed in the process described by Daufresne.

\section{CAuses of ERror}

When the rules laid down by Dakin and by Daufresne for the manufacture of hypochlorite of soda are followed, 
the solution fulfils all the desired conditions. Experience has shown us, however, that in the various hospitals where Dakin's solution is said to have been in use, they often employ under this name various mixtures, more or less dangerous. These defective solutions, which do irritate tissues and do not sterilise wounds, are the result of more or less clumsy faults in technique.

Ist. The worst error consists in attributing to eau de Javel or Labarraque's liquor mixed with a certain proportion of boric acid, the properties possessed by Dakin's solution. A certain number of surgeons are not afraid to use similar solutions. Thus in one large hospital a mixture of Labarraque's liquor and 40 per cent. boric solution was employed under the name of "Dakin's Solution." It is perfectly certain that solutions of which one does not know the content, either of the alkali or the hypochlorite of soda, are useless or dangerous.

2nd. Other errors crop up when hypochlorite of soda solution is prepared according to Dakin's method, but by means of chloride of lime of which the content of active chlorine is not known. The result is that the proportions of carbonate and bicarbonate of soda are no longer exact, and the product obtained is no longer Dakin's solution. Therefore it is indispensable to verify always the titration of chloride of lime ; and, the solution once obtained, to titrate the quantity of hypochlorite which it contains and to apply the phtalein test according to Dakin's technique. Hence, errors in the mode of preparation result in solutions which are irritating because they contain too much alkali or too much hypochlorite of soda; or which fail to sterilise wounds because the amount of hypochlorite of soda is too small, 


\section{IO8 TREATMENT OF INFECTED WOUNDS}

or which do not keep well because they are charged with too much boric acid.

3rd. Mistakes may be made in the way in which the solution is kept. Should hypochlorite of soda be kept in small quantities exposed to light and heat, the strength of the solution rapidly lessens. We have seen in use in a hospital, a solution whose hypochlorite content had diminished to nearly 0.05 . These mistakes are readily avoided by using fresh solutions, or rather by taking pains to keep the solution in darkness and in a cool place. It is prudent to make titrations of the hypochlorite of soda from time to time.

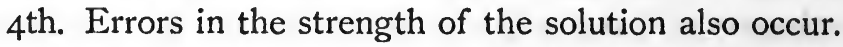
In certain hospitals we have seen a solution used whose hypochlorite content was correct, but whose strength was reduced by addition of water. Solutions thus obtained have a bactericidal potency far too feeble, and they must not be used. Since, as the result of numerous experiments, it has been determined that a solution varying from 0.45 to 0.50 per cent. has no irritating action on the tissues when used under the conditions previously described, Dakin's solution pure and simple should be employed. There is no danger when it is accurately prepared. It is important to make sure that the details of the method previously described have been followed to the letter, if it is desired to obtain Dakin's solution with its characteristic properties. Furthermore, the procedure for sterilisation has been calculated with a view to the application of a liquid possessing the strength and qualities of Dakin's solution, so that any alteration in the solution robs the method of its precision and its efficacy. 


\section{CHAPTER III}

THE TECHNIQUE OF THE STERILISATION OF WOUNDS - MECHANICAL, CHEMICAL, AND SURGICAL CLEANSING

THE first stage of treatment consists in preparing for the penetration of the liquid by surgical interference and by mechanical cleaning of the wound. This intervention is indispensable, in order that intimate contact between antiseptic and microbe may be established. It differs only by some details from the methods in general use to-day.

\section{The Time for Mechanical Cleansing}

Surgical interference and mechanical cleaning-up of a wound are practised as soon as possible after the infliction of the injury. The time for interference is of the greatest moment, for the surgical proceeding has a gravity varying according to the stage of infection in which it takes place.

I. Every infected wound at first goes through a stage which might be termed pre-inflammatory, during which the various local symptoms are very slight or nonexistent. Muscles and cellular tissue preserve their normal appearance. So far there is neither swelling of the tissues nor the reddened tracks of lymphangitis. 
The temperature is normal or rises slowly. This stage usually lasts from twelve to twenty-four hours, and is sometimes prolonged to forty-eight hours. During this pre-inflammatory period free incisions and search for foreign bodies or projectiles present no danger. This is the period of the infection, during which all surgical interference should be carried out as far as possible. It is with wound-infection as with appendicitis. Interference during the first twenty-four hours carries with it little danger, and nearly always yields excellent results.

2. At the end of a period varying from twelve to forty-eight hours, and occasionally longer, the inflammatory stage begins. The temperature goes up, and marked symptoms of infection appear on the surface of the wound. These infectious complications present themselves under two aspects, gangrenous or phlegmonous. In the gas form of infection multiple incisions with thorough opening-up do not aggravate the patient's condition, and as a rule allow the progress of infection to be checked. It is not the same with infections of the phlegmonous type, which are due often to the presence of streptococci.

Every one knows the appearance of the phlegmonous wounds. Neither gangrene nor gas is present, but the tissues are infiltrated and painful. Serum pours from the wound. Sometimes there is lymphangitis, and the glands of a limb near the trunk are swollen and tender on pressure. This stage may last several days, and sometimes several weeks. When the patient is in this condition, the surgical measures which might have been practised had the operation taken place 
during the first twenty-four hours are no longer indicated. Free incisions and prolonged search for foreign bodies or splinters might set up septicaemia, or at least aggravate phenomena both local and general. During this anxious period one has to be contented with no more than is absolutely necessary. To operate at this moment is to make the patient run the same risks as a case of acute appendicitis which is operated on after three or four days.

3. When the stage of acute infection is past, and suppuration has commenced, the search for projectiles, shreds of clothing, splinters may be undertaken with far less danger. But osteo-myelitis in some cases has made its appearance, and wound-cleansing cannot be as efficacious as at the outset.

Upon the whole, the most favourable time for any operation called for by reason of anatomical lesions is the pre-inflammatory stage. If the general condition permit, now is the time to carry out without danger any necessary surgical interference. It is the reason why the wounded man should be got as quickly as possible to the hospital, where complete surgical treatment can be carried out.

\section{Technique of the Mechanical Cleansing OF THE WOUND}

A. Pre-inflammatory Period.-As soon as the patient arrives at the hospital (ambulance, Fr.), he is warmed and cleaned up. If needed, treatment for shock is carried out. Then surgical treatment of the wounds is immediately proceeded with. 


\section{I 2}

TREATMENT OF INFECTED WOUNDS

I. Clinical and Radiological Examination.-(a) Notes of the wounds having been taken, their relations to the various organs of the damaged region are examined. The opening of the wound should be neatly trimmed according to its requirements. The fascia is split or torn by the projectile. Muscles present as a hernia or retract to leave a gaping hole. Lastly, blood issues from the wound, either alone or mingled with the fat coming from a fracture, cerebro-spinal fluid, brain matter, urine or fæces. Inspection of the orifice often yields valuable indications of underlying injuries. The surrounding skin may be red and tense. Sometimes a furrow ending at the orifice gives the direction of the projectile. At another part of the limb a cutaneous bruise may be seen without solution of continuity of the skin. Frequently the projectile is found at this spot. The whole region in which the wound is situate is more or less swollen. Occasionally it is puffy around the opening.

In certain cases the whole segment of the damaged limb is swollen and hard. Very rarely is pulsation felt or a murmur heard. This swelling is due nearly always to haemorrhagic infiltration of the inter-muscular cellular tissue, particularly of the posterior aspect of the calf or thigh. It is a lesion which it is important to bear in mind. In fact, serious infections often occur in these layers of connective tissue, whose blood-infiltration may be widely extended and form an ideal culture-ground. Just as often, instead of swelling we find a localised depression between the two orifices. This depression corresponds to a sub-cutaneous section of the muscles by a projectile which has traversed the limb seton-fashion. It is well to have this information before operating, 
because it determines the nature of the surgical interference. Because, if muscles are severed, we may unite the two openings of the seton by an incision at right angles to the long axis of the limb; whereas, if the muscles are sound, the two orifices should be opened up by incisions parallel to the long axis of the limb.

Pain may prove a useful guide. Often a tender spot points out the site of the projectile. The bony skeleton must be examined, not only to recognise a complete fracture, almost always easy to identify, but also to ensure that the splinters of an incomplete fracture should not escape notice.

The circulation and innervation of the distal portion of the limb are equally subjected to careful investigation.

(b) It is indispensable that the casualty clearing station (ambulance, Fr.) should possess a radiological installation to allow of exact localisation of projectiles. We shall not here go into details as to the most useful method of procedure. Simple radioscopy enables us, if we move certain muscles with the finger, or obtain voluntary contraction, to fix the site of projectiles. It is a quick and practical way of localising multiple projectiles.

To summarise, both a general examination and a minute local examination should be made, as much to decide the actual possibility of surgical interference, as to fix its duration and extent. Equipped with this information, we may proceed as quickly as possible to the mechanical cleansing of the wounds.

2. Anaesthesia.-General anaesthesia should always be employed. Ether should be used; chloroform as rarely 


\section{II4 TREATMENT OF INFECTED WOUNDS}

as possible. In certain cases spinal anaesthesia is employed.

3. Opening-up and cleaning a Wound of the Soft Parts. -The skin is sterilised with tincture of iodine. As the cutaneous apertures of entrance and exit of projectiles are too small to allow of an examination of the course taken by the foreign body, they must be enlarged. The extent of opening-up depends upon the depth of the track of the missile. The eye must be able to survey the whole extent of the wound, especially when fracture exists. The incisions, therefore, are as long as may be needful, and parallel with the long axis of the limb or the fibres of the underlying muscles. As a matter of fact, the track of the bullet nearly always goes through the muscles we are intending to clean, and which must be cut as little as possible. The muscular track, therefore, is laid open by an incision as wide as the skinopening. We do not insist upon the necessity for respecting vessels and nerves. In the case of a blind track, if it does not suffice to lay open the orifice, a counter-opening should be made, which will permit examination of the whole extent of the wound.

In wounds of the "seton" type, the two orifices are laid open separately, parallel with the long axis of the limb, so that the entire track is plainly visible. If this seton-type of wound is superficial, it is sometimes advisable to lay it open from one orifice to the other. Should muscles be severed by the projectile it is preferable to open up the wound completely, in order to clean it the more thoroughly.

There is no call for hesitation in making very free incisions, because they can be brought together again 
after a few days. Extensive opening-up of soft parts nearly always yields earlier closing.

(a) The bruised portions of the track are carefully excised. To Depage and the surgeons of his school is due the merit of having shown how useful it is to resect almost the whole of the walls of the wound. The skin which surrounds the opening, the sub-cutaneous cellular tissue, the superficial fascia, and above all, the muscles in the first third of the track, are almost always riddled with threads of wool or cotton from the clothing. These shreds are embedded in the tissues. No amount of sluicing or swabbing is capable of getting rid of them. They can only be removed by removing the tissues themselves. This line of conduct is all the more justified by the fact that muscular or cellular tissue thus impregnated with tiny foreign bodies is certainly destined to necrosis and elimination.

The mechanical cleansing of a wound, therefore, commences by removal of the skin which adjoins the orifices, of the sub-cutaneous cellular tissue fouled by fragments of clothing and often infiltrated with blood, and of the muscular track encrusted with foreign bodies. The muscular wall is resected to a thickness of about two millimetres over almost the whole extent of the wound. This cleaning with a cutting instrument is much to be preferred to manœuvres which injure tissues without cleansing them. It is no use sponging a track with a gauze swab, introduced by one orifice, pushed to and fro, and then removed by the other opening. This kind of cleansing is always ineffective and harmful, for it inoculates healthy tissues throughout the whole extent of the wound, and produces lesions which 


\section{II6 TREATMENT OF INFECTED WOUNDS}

may be followed by necrosis. Indispensable manipulations, such as the repeated pressure of gauze compresses on a wound-surface to check haemorrhagic oozing, or the use of metallic retractors, have already bruised the tissues. Rough handling, likely to aggravate pre-existing injuries and increase tissue-infection, must be carefully avoided.

(b) Haemostasis. - In the course of the operation, the organs, vessels, and nerves in the neighbourhood are examined and haemostasis of the track completely established. When injury to a large vessel is found in the track of a projectile, it is most necessary to see that adjoining cellular interspaces have not been opened up and infiltrated with extravasated blood. This lesion is common on the posterior aspect of the thigh and calf. In fact, in the sheath of the sciatic nerve, under the biceps, semi-membranosus, and semi-tendinosus, haematomata are sometimes found, infiltrated in the connective tissue which separates the different muscles. The same thing occurs in the calf, near the soleus, gastrocnemius and flexors. There must be no hesitation about laying open these spaces from one end to the other, for infection spreads there with the greatest readiness, and may become of extremely grave character. Incisions are made in such a way as not to endanger the circulation of the part.

(c) Search for and Extraction of Projectiles and Shreds of Clothing:- The difficulties of searching for projectiles are due to the dimensions, sometimes extremely small, of the foreign bodies, to the thickness of the muscular stratum in which they are embedded, and to the irregularity of the course of the projectile through the tissues. 
When a wound is cleansed some hours after infliction, and the foreign body is as large as a small nut, it is generally easy to find it. The muscles which surround the track seem struck by paralysis. Eye and finger follow the route of the missile all the more readily when radiography has indicated the direction of the track. One always tries to arrive at the projectile by means of the track, because it has to be followed and the whole wound cleaned. However, if the track is too long, it is easy to make a counter-opening in the immediate neighbourhood of the projectile. This counter-opening not only allows the projectile to be extracted, but also the inspection of the wound to be completed, and this part of the track to be resected. The various apparatus for registration, and Bergonié's electrovibrator should be made use of. Sometimes the minute fragments of shell are very difficult to locate. In fact, the openings they leave when traversing fascia are very small. Often these may be identified, but directly afterwards the track through muscular fibre is lost. Hirtz's or Contremoulin's compass may prove of use. But when the shell-fragments are numerous and close together, the multiplicity of points registered on the skin is bewildering. Then is the time to call in the aid of the telephone vibrator of M. de la Baume-Pluvinel. This apparatus enables us to find the tiniest fragments.

It is much more important to remove shreds of clothing than projectiles. As a rule, the missile is wrapped up in the fabric it has carried along with it, but sometimes it has only pushed the cloth in front. By the aid of dissecting forceps, every particle of fabric which is found on the surface of the wound is removed with minute care. 


\section{8 TREATMENT OF INFECTED WOUNDS}

The toilet is completed by washing both wound and adjoining skin with neutral oleate of soda.

(d) Drainage.-Drainage of the wound should be liberally arranged, but by a procedure different from what is usually employed. Counter-openings are not made at dependent points. In fact, the antiseptic solution must come into contact with the entire surface of the tissues, and consequently fill the wound. The liquid must not be allowed to escape through the bottom. We shall even see, later on, that when a wound is being drained naturally through a dependent opening, the inferior orifice should be plugged by a tampon. Therefore we have to be contented with freely opening the wound by one or more long incisions, situate as much as possible on the anterior aspect of the limb. The openings thus made are kept gaping by means of compresses placed in the mouth of the wound, or short lengths of very large rubber drainage tube. Compresses or tampons are never placed in the interior of the wound.

When the wound has been thus prepared, and haemostasis is complete, the tissues look quite clean. However, we are never quite sure of having cleansed the wound absolutely. There is no known method of ascertaining the bacteriological condition of a fresh wound while it is still bleeding. The "smears" which would immediately inform us as to the state of wounds more than twenty-four hours old, and from a non-bleeding surface, are of no use at this stage. Cultures give no results before the end of twenty-four hours. And even a negative culture would not signify that the wound was not infected. In reality, in fresh 
wounds, microbes are localised at certain points, and if the specimens are not taken from these points, the tubes remain sterile. Therefore we must refuse, absolutely, immediate closure of a wound, however satisfactorily clean its appearance. As it is impossible to ascertain precisely its state as to infection, the patient would run grave risk if it were sutured. Often has disaster followed premature closing of wounds.

4. Cleansing of Compound Fractures or Wounds of Joints.-(a) Cleaning-up a Compound Fracture.-The incisions for exploration and cleaning-up of compound fractures should always be very free. A long incision is no drawback, because it can be sutured two or three weeks later. Whenever possible these incisions are made on the anterior aspect of the limb in such a way that the liquid may remain in contact with the bony fragments. Counter-openings at the dependent points are not made. Soft parts are laid open in such a manner that all parts of the seat of fracture may be explored. In fractures of the femur, it is peculiarly important to make an incision so long that the masses of muscle can be retracted sufficiently to lay bare the fissures in the bone, however long they may be. These long incisions should be kept open. Muscular masses have a marked tendency to reunite in such a way that the seat of fracture becomes shut off. The opening can be kept gaping by means of short pieces of rubber tubing, three centimetres in diameter, which are kept separate from each other by a second set of tubes at right angles. Those haematomata which form along the sciatic nerve and in the sheath of the femoral vessels, about the popliteal space and along the posterior tibial vessels, 
must be reckoned with. Whenever found in this condition, these sheaths must be opened, because they are protected from the antiseptic liquid and become startingpoints of infection. Exploration of the soft parts sometimes brings to light tiny splinters which have perforated the muscles. These are removed at the same time as the lacerated portions of muscular tissue.

Splinters are often found lying free between the fractured extremities and in the medullary canal. These splinters are removed. The medullary canal is explored, and in the case of longitudinal fractures, the marrow is removed. All splinters adherent to the periosteum are preserved. Experience has shown, in fact, that fractures so treated become sterile, heal without sinuses, and rapidly consolidate. And, on the contrary, the extensive removals of splinters which too often have been practised in the "ambulances" at the front, have yielded deplorable functional results. Even very serious injuries of the bones should not be followed by immediate amputation, except in the cases of extensive smashingup of the skeleton, or of destruction of vasculo-nervous bundles. Careful cleansing, as conservative as possible, should be made of the multiple seats of fracture, with the object of placing the conducting tubes in contact with bony surfaces. Thus it becomes possible to save many limbs which otherwise would be condemned to amputation.

Most careful haemostasis is practised. But avoid leaving compresses in the deeper parts of the wound, or only leave them there for a few hours.

(b) Cleansing of Foint-injuries.-Wounds of joints are treated in different ways, according as the synovial 
membranes are alone concerned, or the bony extremities in addition.

When synovial cavities are alone concerned, the projectile is extracted, and the joint emptied of the blood it contains. The contaminated region is isolated from the rest of the joint cavity by compress or suture, and the instillation tube is placed in the situation previously occupied by the foreign body.

If the bony lesions consist simply of a chafing of the surface, or perforation of one of the extremities by a projectile, or an unimportant fracture of an epiphysis, the course to take is almost identical with that we have just described. The only addition is to scrape the bony surface which has come into contact with the projectile or with shreds of clothing. This region is cut off as completely as possible from the rest of the articular cavity, and submitted to instillation of the antiseptic liquid.

Should the bony lesions be very extensive, it becomes necessary to perform a resection. But primary joint resections are to be made with circumspection. Because, chemiotherapy often allows repair of extensive lesions of articulations, which, under any other treatment, would have had to undergo resection of the osseous extremities.

B. Inflammatory Period.-This stage may begin about six or eight hours after the incidence of the wound. But usually it starts towards the twenty-fourth or thirty-sixth hour, sometimes not until after the lapse of several days.

Two quite different classes of phenomena are observed: gangrenous infections and phlegmonous infections. The 


\section{TREATMENT OF INFECTED WOUNDS}

first are of early onset and rapid progress. The second are slower to appear, more tardy in evolution. Both types of infectious manifestation may coexist in the same wound. Their symptoms have been described by the classic authors. But their pathological physiology is little known. Only it is recognised that the general reaction following surgical traumatism is much more violent during the inflammatory period than during the pre-inflammatory stage. Manipulation and laceration of tissues may set up grave complications when microbes already swarm in the walls of the wound. We have seen cases operated on at the expiration of several days for a localised infection, present signs of septicaemia and die after this interference with the focus of infection. At the beginning of the campaign, tetanus at times occurred a few hours after such operations. And when the nature of the infection was less alarming, still the general condition of the patient remained worse than before, and his temperature chart showed great fluctuations for several days afterwards. Hence, whilst the toilet of a war-wound should be carried out in minute detail before the advent of inflammatory phenomena, it is prudent to confine oneself to what is strictly necessary, during the stage of confirmed infection.

The course to adopt varies according as the infection is of the gangrenous or the phlegmonous type.

I. Gas-producing Infection.-Gas-gangrene presents itself under three different forms: the septicaemic type, the grave local type, and gas-cellulitis.

(a) The septicaemic form is particularly frequent in fractures of the femur with serious muscular laceration. 
After a few hours the patient has nausea and vomits. $\mathrm{He}$ is agitated. The pulse is rapid, small, indistinct. However, the patient does not yet complain of great pain in his limb, and there is little gas to be discovered. This appears, clinically, several hours later than the general phenomena. Death comes before the limb has had time to necrose. Amputation is urgent, to have even a feeble chance of saving the patient's life.

(b) Local gas-producing infection, which does not act at the very outset on the patient's general condition, if suitable treatment be adopted, is the most frequently recovered from. Two principal forms may be distinguished, a superficial and a deep form. The superficial gangrene evolves chiefly in the sub-cutaneous cellular tissue. Gas rapidly spreads, far from the site of trauma. Opening-up shows that cellular tissue almost alone is invaded, and that muscles are not gangrenous, save in the immediate neighbourhood of the wound. This form is fairly benign. Numerous incisions implicating at the same time both the skin and the superficial fascia are made wherever crepitation can be felt. Tubes are placed in each incision.

Deep gangrene involves more particularly the muscles. Pain and agitation are often the earliest symptoms. Pain extends in the direction of the trunk, along muscular sheaths. It is the path which the infection itself has followed. If the limb is not yet completely necrosed, it is needful, after having set free the muscles attacked, to open up vascular sheaths. When the muscles of the calf are attacked, the femoral sheath should be incised between Scarpa's triangle and Hunter's canal. Finally, all around the limb are made incisions about ten 


\section{I24 TREATMENT OF INFECTED WOUNDS}

centimetres (four inches) long, including both skin and fascia. This local form may call for amputation. If muscles are found to be gangrenous, and in addition the vessels obliterated, it is prudent to remove the limb.

Amputation is practised at a short distance from the seat of injury. Moreover, the vascular sheath must be laid open, in order to make sure that infection has not already invaded it. The stump is left quite open. A tube, perforated with small holes in its middle third, is placed loop-wise on the stump (Fig. 51). At the same time, instilling tubes are placed in the vicinity of the vascular sheaths. After an amputation of the thigh, three tubes are used-for the internal saphenous, the femoral vessels, and the profunda.

(c) Localised Gangrene.-This is a benign form of gas-producing gangrene. It is often found localised in a muscular sheath. For example, it may be limited to the anterior muscles of the leg, or the peroneal muscles. It may even affect only part of a muscle. To lay it open freely will suffice, the incisions extending beyond the lesion in every direction. Then the instillation tubes are placed in position, care being taken to lead them into muscular interstices and into the muscles themselves. The course of local gas-gangrene, under the influence of hypochlorite of soda, is very favourable. Swelling and redness disappear, the junction of the limb with the trunk remains supple and free from oedema, the patient is no longer in pain, and his general condition is excellent. Elimination of mortified tissues takes place very quickly, because hypochlorites dissolve necrosed muscle. Often by the seventh day, there is no longer a trace of gangrenous tissues. 
A still more benign form of gas-producing infection exists, gas-abscess. A simple incision will suffice.

2. Phlegmonous Form.-The clinical aspects of the phlegmonous form are extremely varied. Reticular lymphangitis may be seen around a superficial wound, or a line of inflammation of a lymphatic trunk extending to the proximal extremity of the affected limb, or a serious local inflammation with redness and great swelling of the limb, or slight inflammation coincident with a grave general condition. In the case of lymphangitis of either variety, the wound is sterilised by Dakin's solution, and a hot fomentation applied over the limb. If a lymphangitic abscess should form, it is incised and the cavity sterilised by Dakin's solution.

When the muscles are concerned in the injury, and the phlegmonous inflammation extends to the whole thickness of a muscle-group, it is necessary to lay open the focus of inflammation, and also the intermuscular spaces in which the infection is being produced (generally due to haematoma). But surgical interference should be limited to this. It is not wise to seek for projectiles or foreign bodies, nor to remove the splinters from a seat of fracture. In these highly infected wounds, meticulous exploration is more dangerous than useful. The seat of fracture is kept freely open, and into every diverticulum is inserted an instilling tube. It is dangerous to use the scalpel to wounds from which blood-stained serum is coming. An attempt must be made, in the first place, to lessen the infection by antiseptic treatment. If a tube instilling hypochlorite can be introduced into the track resulting from a previous operation, it is well to be content with this therapeusis. Perhaps it may be needful 


\section{I26 TREATMENT OF INFECTED WOUNDS}

to lay open a wound still more freely in order to introduce the tube which will supply the antiseptic liquid. Then an incision is made in which one or two tubes are placed quite in the bottom of the track, without further traumatism of the tissues. At the same time, rigorous immobilisation of the limb is insisted on.

To resume, the treatment of a patient with a phlegmonous wound differs from the treatment of a case in the pre-inflammatory stage. Preventive therapeusis of infection calls for minute surgical cleansing, which at that stage of infection presents no danger. But when, on the contrary, infection is well established in a wound, it is necessary 'in the first place to check it by the simplest means at hand, and to postpone to a more favourable opportunity the surgical treatment called for by anatomical lesions and the presence of projectiles.

Some modifications have to be made in this technique, due to the nature of the injury.

(a) Infected Fractures. - The course to pursue in compound fractures, the seat of acute diffuse inflammation, is similar to that we have just laid down for wounds of the soft parts. Only what is strictly necessary is done in the first place; that is to say, simple laying open of a seat of fracture without minute cleansing, and the placing of several instillation tubes in the diverticula of the wound. After a few days the general condition improves. Swelling, redness, pain, diminish. Then, when the dangerous stage of infection is passed and the number of microbes per field of the microscope remains considerable, the toilet of the seat of fracture is made. This new interference is as complete as possible. Foreign bodies, carefully registered by suitable 
apparatus, are removed at the same time as the splinters, but the periosteum of the splinters is preserved with care. The operation ends by arranging in the seat of fracture multiple tubes destined for supply of the antiseptic solution.

(b) Suppurating Foint-injuries.-In arthritis without bony lesions, arthrotomy more or less free, followed by the extirpation of foreign bodies and cleansing of the articulation, suffices generally to ward off evil results, if the antiseptic treatment be carefully employed and the joint immobilised absolutely.

In joint-injuries with bone lesions intervention is limited to the measures which, aided by chemical sterilisation, check the spread of infection. The general condition of the patient, the nature and the virulence of the infection, play an important part. Streptococcal infections are the most grave, and call for more extensive interference than the other infections. In these cases, sometimes, the prospect of amputation must be faced.

(c) Secondary Haemorrhage.-Haemorrhages are often due to the detachment of a scar produced by contusion of the wall of a large arterial trunk. But they arise also from the breaking down of clot, which had previously brought about spontaneous haemostasis of a wound of an artery or vein. The clot disappears under the influence of infection, and the artery finds itself more or less widely open. In this manner a primary haemorrhage is produced, perhaps only slight, but which is followed some days later by a loss of blood much greater, often mortal. Haemorrhage may also follow the loosening of a ligature, silk being readily dissolved by hypochlorite, as Fiessinger has shown. That is why we ligature 
vessels with catgut or chromic catgut. When these precautions are taken, haemorrhages are never observed.

The preventive treatment of haemorrhage consists in careful examination of the vessels at the time of surgical interference, and in bringing about definite haemostasis if a vessel be wounded.

When a case presents a primary haemorrhage, most frequently a tampon will stop the bleeding. But several days later a new haemorrhage will not fail to appear, and the patient may succumb. It will not do to be content with a tampon; ligatures must be used above and below the injury, and as near as possible to the seat of ulceration.

Haemorrhages have occurred in certain hospitals after using badly prepared Dakin's solution. The solution then contains free alkali, which is just as capable of producing vascular ulceration as eau de Javel or Labarraque's liquor.

In wounds chemically sterilised the classic secondary haemorrhages due to suppuration are never seen.

(d) Wounds of the Brain.-For wounds of the brain the projectile should be removed, whenever possible, through its orifice of penetration. It is important to avoid causing traumatisms of the cerebral substance by rough manipulations or by washing out the wound by means of a liquid under pressure. The walls of the wound are freed as thoroughly as possible from all foreign bodies which may be found in them. As it is of the highest importance to the future of the patient that the wound should be absolutely sterilised, all its parts should be brought carefully into contact with the antiseptic. It is therefore essential immediately after 
operation, to introduce a special appliance which will permit of its sterilisation, and as far as possible this appliance must not be removed until the bacteria have completely disappeared.

C. Suppuration Stage.-The manipulation of wounds which have arrived at the stage of suppuration is effected with all the more precaution because still nearer the inflammatory stage. Two extreme types of suppurating wounds may be present. The first type is the wound covered with pus more or less blood-stained, accompanied by lymphangitis, swelling and pain. It is the transition period between the inflammatory stage and the period of true suppuration. Unless there are urgent indications to the contrary, these suppurating wounds must be treated with as much respect as wounds in the inflammatory period. The other type is represented by wounds of longer standing. From the orifice, already covered by granulations, thick "laudable" pus escapes. The tissues are no longer oedematous. The temperature is only slightly raised, or presents great variations. At this stage it is possible to interfere surgically with less danger than in wounds of the first type. Between these two extreme types a number of intermediate conditions are found. Surgical interference becomes less and less dangerous as the wounds are removed further and further from the first type. In a general way the cleansing of the wound follows the same rules in all cases; the more inflamed the wound the more sparing should be surgical interference.

Ist. Chemical Cleansing.-In the great majority of cases the wounded who arrive at the hospital at the end of two, ten, or fifteen days have already been operated 


\section{30 TREATMENT OF INFECTED WOUNDS}

upon. On the surface of the limb, therefore, openings are found leading down to the solutions of continuity in soft parts, to opened joints, to seats of fracture. These openings are often too small, and inadequate to drain the pus-laden burrows. Nevertheless, it is better not to interfere at the outset. Even at this stage it is hardly wise to open up an abscess. It is enough to remove the drainage tubes which generally have been placed in the wound, and replace them by the small instillation tubes which are gently coaxed into the orifices alreads in existence, down to all the diverticula of the soft parts and to the seats of fracture. This is done without anaesthesia and without distressing the patient. Then Dakin's solution is instilled, according to the method which will be described later, until suppuration ceases, temperature drops, and the general condition improves. From the clinical point of view, suppuration disappears after the lapse of a space of time varying from twenty-four hours to about four days.

2nd. Surgical Cleaning.-After a little time, in wounds accompanied by injuries to bone, the amelioration resulting from the application of the antiseptic is arrested. The number of microbes found on the surface of the wound remains stationary. But, on the other hand, suppuration has diminished or dried up, the tissues are no longer swollen, and the patient is ready for surgical intervention.

Then the wound is cleansed just as though it were a fresh one. Under anaesthesia, foreign bodies and necrotic tissues are removed.

In the case of suppurating fractures, the method of procedure is slightly different, according as intervention is practised before or after consolidation. This point 
was recently cleared up at Compiègne by the researches of MM. Guillot and Woimant.

(a) Before Consolidation.-It is an easy matter to explore the bony extremities. Incisions will not necessarily be made in already existing wounds. Whenever it is possible to do so without involving a fresh and extensive traumatism, it is best to choose the classical paths of surgical access, while avoiding those which, being in a sloping position, are not very favourable to irrigation. Between the bony extremities, or in their neighbourhood, will be fragments of necrosed bone and splinters. The former having been removed, the latter will be sacrificed only if they show signs of necrosis, or if they hinder the inspection of the seat of the fracture, and its irrigation. The bony extremities will be carefully examined, for the result of the operation is directly dependent upon their treatment. When they are filled by a medullary plug, it is necessary to make sure, by scraping the latter with the curette, that it is healthy, without fissures or enclosed sequestra, in which case it is certain that the underlying medullary cavity is practically sterile. In the contrary case it is necessary to remove the whole thickness of the plug, and to provide for drainage, by scraping a groove in the dense tissue for a depth of twofifths of an inch. The same procedure will be followed in the case of bony extremities which are left opening into the seat of the fracture. This precaution of opening up a wide passage for the irrigation tubes in the dense tissue is of the greatest importance if we wish to avoid violent post-operative febrile reactions.

(b) After Consolidation. - The exposure of the bony extremities presents more difficulty. In the first place, 


\section{I32 TREATMENT OF INFECTED WOUNDS}

the path of access is obstructed by fistulas which have to be resected, and the formation of callus. When the orifice of an osseous fistula is reached through the soft parts, the periosteum is detached from the callus by means of a sharp rugine, taking care not to denude it too far. The curette then enlarges the fistula, enabling the bone-forceps to come into play, and permitting of the resection of the periosteal callus and the fragments, which is necessary to the cleaning-up of the cavity of the seat of fracture, and the exploration of the medullary cavities. The treatment of the bony extremities thus exposed will be the same as before consolidation.

The capital point in the operative treatment of old fractures inclined to suppurate is to practise complete but economical operation. In short, the abrasion of the bony tissue must be compatible with the reunion of the soft parts after the few days necessary for sterilisation.

It is obviously possible to treat old fractures in a single surgical operation by an extensive sub-periosteal resection, going considerably beyond the boundaries of the lesion in both directions. But this method of procedure is incompatible with secondary reunion; it must be regarded as a surgical technique which was excellent in times when one could not imagine the reunion of infected wounds.

In a case of suppurative arthritis, if necessary, resection of the bony extremities is practised. At this stage the surgical interventions found absolutely necessary may be carried out with much less danger than when the patient "came in." It must be borne in mind, however, that tissues which have already commenced to cicatrise during the stage of suppuration are impregnated 
with microbes and that reinfections are possible. Therefore operations involving the least possible amount of traumatism should be chosen.

Wounds of the soft parts, as a rule, become aseptic under the influence of the antiseptic without a new operation being necessary.

3rd. Chemical sterilisation.-The surgical cleaning-up is followed by the introduction of instillation tubes precisely as though dealing with a fresh wound. It is necessary to keep the wound gaping so long as its deeper parts are not sterilised. This result is attained by placing in the wound short segments of tube of wide calibre, by the side of which are introduced the small tubes for instillation.

In the case of fractures operated on during the suppurative stage, MM. Guillot and Woimant have shown that certain precautions are necessary in order to limit post-operative reactions. Our aim should be to deprive the bacteria of the media of culture which are favourable to them: blood-clots and contused tissues. The blood-clots are avoided by a thorough haemostasis with the forceps, which is completed, as regards the osseous and medullary oozing, by a prolonged irrigation with warm serum. The rapid elimination of the contused tissues will be achieved, on the one hand, by an abundant irrigation performed at short intervals, every hour or even every half-hour, if an automatic apparatus is available.

D. Cicatricial Stage.-The cicatrisation of a wound does not mark the end of infection. In fact, microbes remain included in the cicatricial tissue. Therefore secondary interference practised on a patient whose 


\section{I34 TREATMENT OF INFECTED WOUNDS}

wounds have healed, during a period of suppuration more or less long, is subject to special rules. Every one knows that after stump-trimming, nerve-suture, osteotomy for defective union, suture for pseudarthrosis, etc., infections, sometimes most alarming, may arise. It is therefore prudent, in these secondary interventions, to refrain from suturing the wounds, and to place in the deepest parts one or two tubes carrying the antiseptic liquid. The sterilisation of operation wounds is thus rapidly obtained, and the accidents due to reinfection avoided. In bone-grafting, the extremities of the bone are prepared for the reception of the graft, and in the wound thus created instillation tubes are placed. After a few days, it is ascertained that the wound is actually aseptic, and then the grafting is completed and the soft parts closed.

In a word, during the cicatricial stage, surgical interference practised in two stages, which are separated by a period of disinfection, is the surest means of avoiding disaster. 


\section{CHAPTER IV}

THE TECHNIQUE OF THE STERILISATION

OF WOUNDS-CHEMICAL STERILISATION

Chemical sterilisation of a wound is brought about by instillation, continuous or intermittent, of an antiseptic liquid, by means of small rubber tubes, into all the recesses of a wound. As the quantity of liquid used is very small, it is not necessary to employ drainage tubes or to arrange for reception of an overflow. The liquid which has moistened the tissues is absorbed by the dressing and evaporates. Instillation thus practised permits of the continual renewal of the liquid over every portion of the wound. This procedure differs from the old "irrigation," in that it is much simpler, and in that the liquid is carried directly to the deepest diverticula of the wound.

\section{Conducting Tubes and Reservoirs}

A. The Conducting or "Instillation" Tubes.-The conducting tubes are of red rubber. The rubber wall of the tube has a thickness of $\mathrm{I} \mathrm{mm}$., and the interior diameter is $4 \mathrm{~mm}$. They are thus resistant and flexible. These qualities allow of their penetration to every irregularity of the wound, and of their adequate resistance to 


\section{I36 TREATMENT OF INFECTED WOUNDS}

the pressure of muscles and dressings. Three kinds of tubes are used.

Ist. Tubes perforated with Small Holes.-The length of these tubes varies from 30 to $40 \mathrm{~cm}$. (roughly 12 to I6 inches). Some of them are closed at one end by a ligature, and pierced by small holes over a length of from 5 to 20 cm. ${ }^{1}$ from the closed extremity (Fig. 35). The

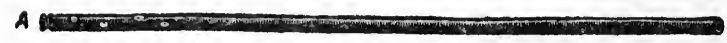

$\boldsymbol{\theta}$

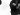

0

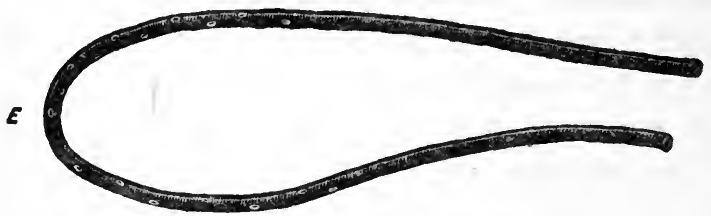

FIG. 35.-Conducting or "instillation" tubes, rubber, with multiple holes, closed at one end.

A. Tube $30 \mathrm{~cm}$. long, pierced over a length of $5 \mathrm{~cm}$.

B. Tube $30 \mathrm{~cm}$. long, pierced over a length of $10 \mathrm{~cm}$.

C. Tube $40 \mathrm{~cm}$. long, pierced for a length of $15 \mathrm{~cm}$.

D. Tube $40 \mathrm{~cm}$. long, pierced for a length of $20 \mathrm{~cm}$.

E. Tube open at both ends, and pierced over a length of $20 \mathrm{~cm}$. in its median portion (ro $\mathrm{cm}$. = about 4 inches).

holes number about eight to each $5-\mathrm{cm}$. section. Their diameter is about half a mm. ${ }^{2}$ The holes are made by means of an ordinary punch. These tubes are the most used. Four different categories are in use, according as the holes are perforated over a length of 5, 10, 15, or $20 \mathrm{~cm}$. Other tubes are left open at each end and

${ }^{1}$ Say 2 to 8 inches.

2 to th of an inch. 


\section{TECHNIQUE OF THE STERILISATION I 37}

pierced with holes only in their middle third (Fig. 18, E). Liquid enters by each end.

2nd. Tubes with a Single Opening.-These are of a length of from 25 to $30 \mathrm{~cm}^{1}$ and the ends are open (Fig. 36). At half a centimetre from one end a large

FIG. 36.-Conducting or " instillation" tube with terminal opening. Tube of about $30 \mathrm{~cm}$. long, open at both ends, with a lateral opening near one end.

lateral opening is made. This lateral orifice is intended to permit the egress of liquid should the terminal orifice become blocked.

3rd. Tubes perforated with Small Holes and covered with Absorbent Fabric.-These tubes are closed at one extremity and pierced with small holes over a variable length. The section pierced with little holes is covered with a sheath of fabric similar to the material of which bath towels are made (Fig. 37). This sheath is firmly

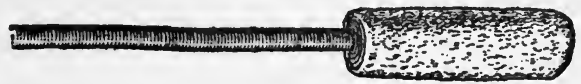

FiG, 37.-Conducting or "instillation" tube covered with a sheath of bathtowelling (tissu eponge).

stitched to the tube. It is intended to distribute the liquid over the whole surface of the tube as it escapes from the holes. It is important that the cover should be so firmly fixed by a stitch to the rubber tube that it cannot remain behind in the recesses of the wound when the tube is withdrawn.

These tubes are of uniform length. They can easily be lengthened to any extent by means of pieces of

1 Say 9 to 12 inches. 


\section{I38 TREATMENT OF INFECTED WOUNDS}

rubber tube of the same calibre and "unions" of pieces of glass tube (Fig. 39, C) of a calibre of $4 \mathrm{~mm}$. and a length of $2.5 \mathrm{~cm}$.

B. The Distributing Tubes.-The tubes pierced with holes are grouped into sets of two, three, or four by

A
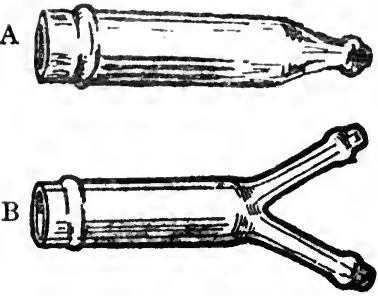

C

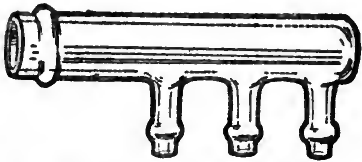

$\mathrm{D}$

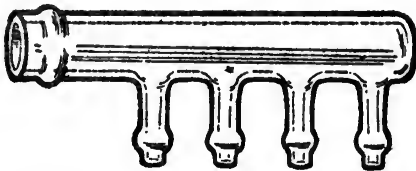

FIG. 38. -Glass distributing tubes

(Gentile).
A. Tube with one branch.
B. Tube with two branches.
C. Tube with three branches.
D. Tube with four branches.

A
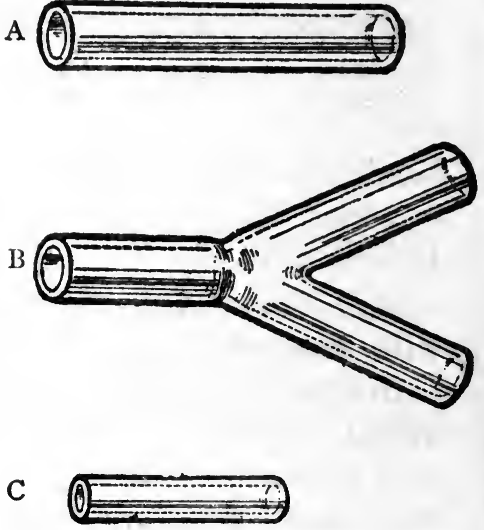

FIG. 39.-Glass connecting tubes, "unions."

A. Cylindrical tube of a length of 4 to $5 \mathrm{~cm}$. and an interior diameter of $7 \mathrm{~mm}$.

B. $Y$-tube with an interior diameter of $7 \mathrm{~mm}$. These tubes unite the ends of rubber irrigating tubes.

C. Cylindrical tube of a length of $3 \mathrm{~cm}$. and an interior diameter of $4 \mathrm{~mm}$. This tube serves to join-up two small conducting tubes, when it is necessary to add to the length of one of these tubes.

means of appropriate branched tubes. Two types of branched tubes are employed (Fig. 38).

Ist. The $Y$-shaped tube is composed of a main stem about $2 \mathrm{~cm}$. long with a calibre of $7 \mathrm{~mm}$., and of two limbs or branches of equal length, about $2 \mathrm{~cm}$., whose interior calibre varies between 3 and $4 \mathrm{~mm}$. (Fig. 38, B). 
Upon the two branches are fitted either two simple instillation tubes, or the two extremities of a tube perforated with holes in its middle portion.

2nd. The distributor with four branches is composed of a glass tube closed at one end, 6 or $7 \mathrm{~cm}$. long, and of a calibre of $7 \mathrm{~mm}$. (Fig. 38, D). From one side of this tube project at right angles four smaller tubes, each of a length of $2 \mathrm{~cm}$. and an interior calibre of 3 to $4 \mathrm{~mm}$. Thus it has the look of a comb. In the same manner one may have three branches (Fig. 38, C), or five or six.

3rd. Small glass connecting tubes or "unions" must also be at hand to join together the rubber tubes of large or small calibre, or to unite a rubber tube of small calibre to one of large. The first are cylindrical glass tubes 2 to $3 \mathrm{~cm}$. long, and of a calibre of 4 and of $7 \mathrm{~mm}$. (Fig. 39, A and C). The others are conical glass tubes of the same length, presenting at one extremity an interior diameter of 3 to $4 \mathrm{~mm}$., and at the other extremity an interior diameter of $7 \mathrm{~mm}$. (Fig. 38, A). Tubes of $\mathrm{Y}$-shape are also in use, of $7 \mathrm{~mm}$. calibre, for joining up irrigating tubes (Fig. 39, B).

C. The Irrigating Apparatus.-The irrigating apparatus is composed essentially of a reservoir (ampoule or flask) fixed at a certain height above the patient's bed, with a tube (equipped or not with a drop-counting contrivance) and stop-cock, so as to allow of either continuous or intermittent instillation.

Ist. The reservoir for liquid usually employed is a flask holding a litre ( ${ }^{\circ} 76$ pint, $0 \cdot 22$ gallon). Its interior, orifice has a diameter of $7 \mathrm{~mm}$. (Fig. 40). To this is attached an irrigating tube of red rubber with a calibre of $7 \mathrm{~mm}$. The flask is fastened to a wooden 


\section{I40 TREATMENT OF INFECTED WOUNDS}

standard firmly fixed to some convenient portion of the bedstead, a portion which depends upon the situation of the wound. It is suspended at a height of from $50 \mathrm{~cm}$. to I metre above the level of the bed.

2nd. The irrigating tube, as we have just said, has an interior diameter of $7 \mathrm{~mm}$. Its length is from $\mathrm{I}$ metre

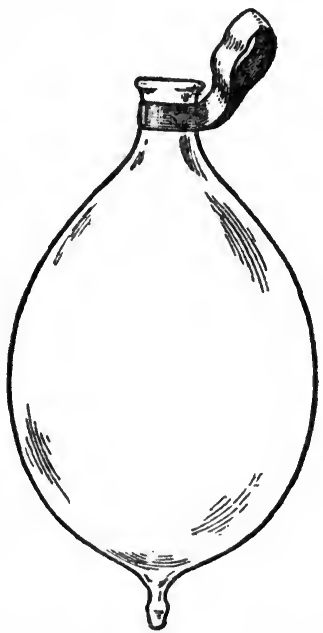

FIG. 40.-Ampoule or flask holding a litre.

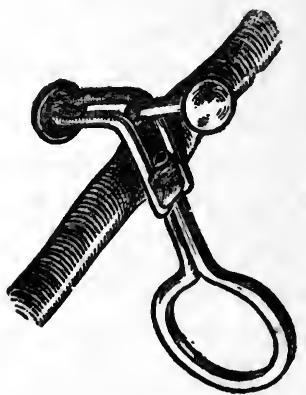

FIG. 4I.-Pinch-cock (Pince de Mohr d ressort).

$50 \mathrm{~cm}$. to 2 metres. Whilst the superior extremity is attached to the flask, its lower end is united with a glass cannula, to which are fixed the smaller tubes which convey the liquid to the wound. At ro centimetres below the flask the tube is furnished with a pinch-cock (Fig. 4I). Slight pressure upon the spring suffices to open the lumen of the tube and to allow the liquid to flow. This apparatus is extremely simple, and well 
suited to the intermittent irrigation of wounds (Fig. 42). Every two hours a nurse stops at the foot of the bed and releases the spring of the Mohr pinch-cock for a few seconds. Instillation at once takes place.

In the hospitals at the front, where it is difficult to

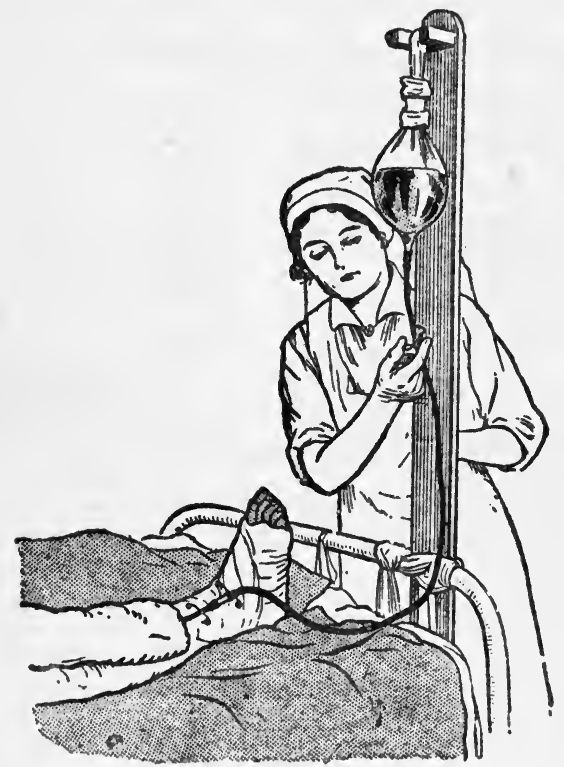

FIG. 42.-Nurse using a pinch-cock and so instilling antiseptic liquid.

provide the needful number of apparatus, the plan devised by le médecin-major Perret may be used. This consists of a support on wheels (dressing wagon) carrying the reservoir of Dakin's solution at the required height. The orderly propels the wagon from bed to bed and injects the liquid into the wounds by means of a cannula, 


\section{TREATMENT OF INFECTED WOUNDS}

which is changed for each patient. This proceeding simplifies the provision of apparatus, but greatly adds to the work of the staff.

The liquid may be instilled also by means of a syringe. The most convenient syringe for this purpose has been made by Gentile. It consists simply of a glass tube drawn out to a fine jet at one end, and of a capacity of 10 c.c. (Fig. 43). The piston is replaced by a bulb of red rubber. The advantage of this syringe is that it can be used with one hand. Each case has its own syringe. It is kept half-immersed in the bottle which holds the supply of Dakin's solution be-

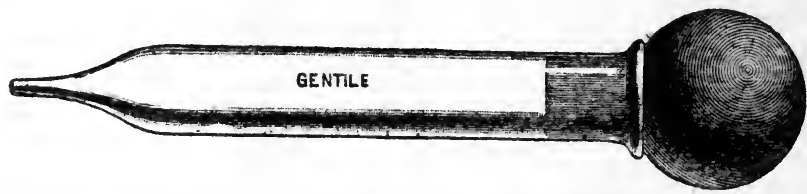

FIG. 43.-Syringe (Seringue de Gentile).

longing to the case. The use of a syringe for the instillation of liquid has also the drawback of increasing the work of the personnel. Besides, instillation done with a syringe gives results far less speedy than with the irrigating reservoir, because the quantity of liquid is much less considerable; and, the tube constituting a siphon, the moment the syringe is withdrawn the liquid immediately runs out of the wound instead of remaining there.

We have completely given up the use of the syringe for instillations. We use Gentile's syringe to test the permeability of the tubes in the course of doing the dressings. 


\section{TECHNIQUE OF THE STERILISATION I43}

When it is desired to practise continuous instillation instead of intermittent, the apparatus is modified after the following manner. To the lower aperture of the flask is attached a rubber tube $10 \mathrm{~cm}$. long. At the extremity of this tube is attached one of Gentile's "dropcounters" (une ampoule compte-gouttes de Gentile). Between the drop-counter and the reservoir is a screw pinch-cock (une pince de Mohr a vis) which enables us to

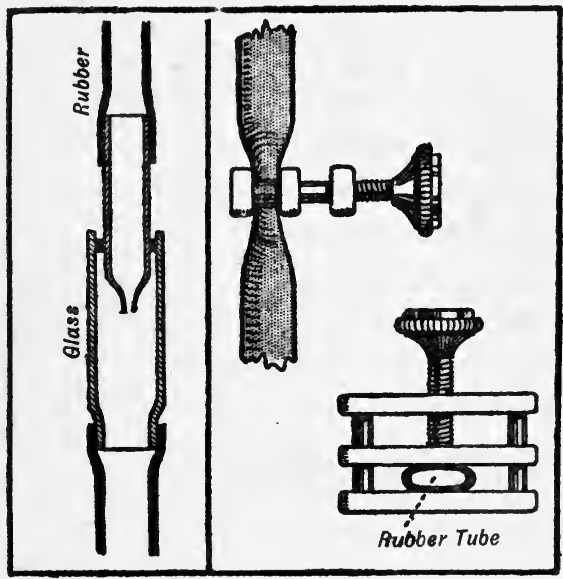

FIG. 44.- " Drop-counter," Gentile's. Screw pinch-cock (Pince de Mohr à vis).

regulate the number of drops per minute which the apparatus should deliver. The lower end of the dropcounter is connected to the irrigating tube (Fig. 44). As the quantity of liquid which traverses a section of tube in a unit of time is very small, it is useless to employ an irrigating tube of diameter as great as that in use for intermittent instillation. A calibre of 5 to $6 \mathrm{~mm}$. is sufficient. 
D. Method of using the Different Tubes and Apparatus. - Ist. The appliance for continuous instillation should never be connected up with several tubes, nor with a tube perforated with several holes. As the output of a drop-counter is very small, all the liquid should flow through a single tube and emerge from a single hole in this tube, the hole and the tube being dependent on gravitation. Consequently, instillation drop by drop should only be used for wounds which contain a single tube, perforated at its extremity (Fig. 45), or a single tube sheathed with "tissu éponge" (bath-towelling).

2nd. The apparatus for intermittent instillation can be connected up with four tubes perforated with tiny holes, or even, in certain cases, with eight. As the yield of the irrigating tube is considerable, the liquid, at the moment when the spring of the pinch-cock is released, spurts out from all the holes of all the tubes. As much as possible, tubes of a length of 5 and of $10 \mathrm{~cm}$. should be used, especially if a single flask furnishes the liquid to eight tubes.

It will not do to serve from the same cannula both simply-perforated tubes and tubes sheathed in "tissu éponge." By reason of the different resistances, the liquid would escape almost entirely by the simply perforated tubes.

It is important to remember this difference in the action of the two forms of apparatus, for continued instillation and for intermittent instillation, because, if a drop-counting appliance be used in connection with a system of general perforated tubes, no result will be obtained. The device for intermittent instillation is used much more frequently than the apparatus for 


\section{TECHNIQUE OF THE STERILISATION I45}

continued instillation, because it allows a single irrigation reservoir to provide liquid for four or eight tubes at once (Fig. 46). It is therefore applicable to all large wounds.

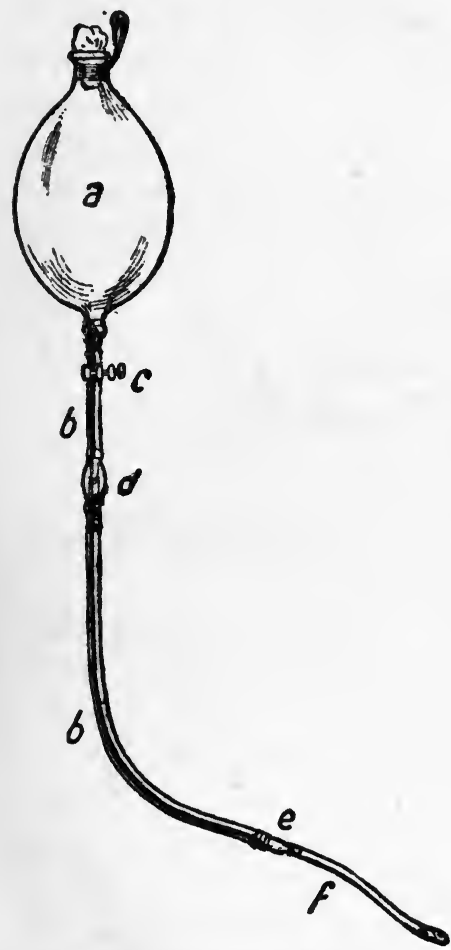

FIG. 45.-Apparatus arranged for dropby-drop instillation: $a$, Reservoir; $b$, Irrigation tube ; $c$, Screw pinch-cock ; $d$, Drop-counter ; $e$, Distributing tube (Fig. $\left.3^{8}, \mathrm{~A}\right) ; f$. Conducting tube with terminal orifice (Fig. 36 ).

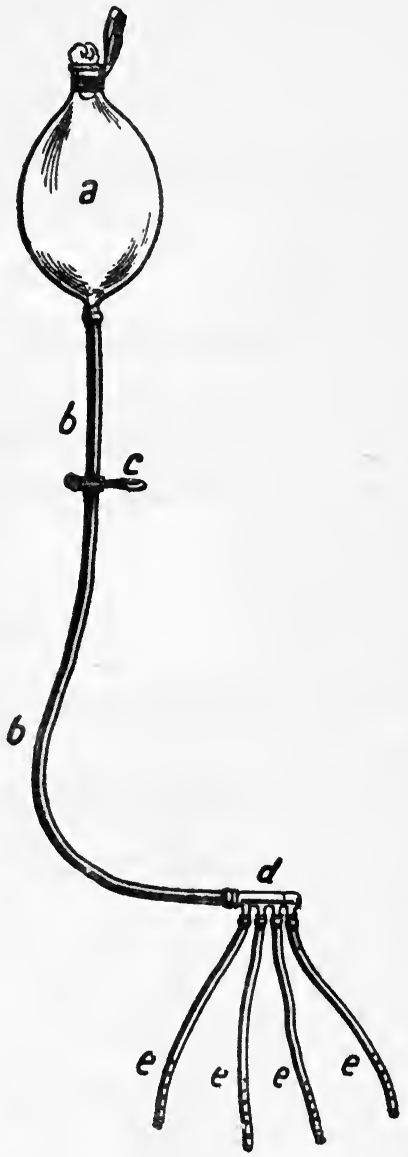

Fig. 46.-Apparatus for intermittent instillation: $a$, Reservoir (ampoule or flask holding a litre); $b$, Irrigating tube with a diameter of $7 \mathrm{~mm}$.; $c$, Pinch-cock (Pince de Mohr) ; d, Distributing tube with 4 branches; $e$, Conducting tubes. 


\section{I46 TREATMENT OF INFECTED WOUNDS}

\section{Arrangement of the Tubes in a Wound}

A. General Principles.-The disposition of the tubes in a wound is such that the liquid may readily spread over the whole surface. As it is essential that the antiseptic liquid should be in contact with the tissues themselves, the tubes are not applied over gauze, or over "wicks," but directly to the wound. In fact, a thin compress placed on the surface of granulations might be supposed to be able to distribute the liquid over the whole extent of their surface. Also it might be

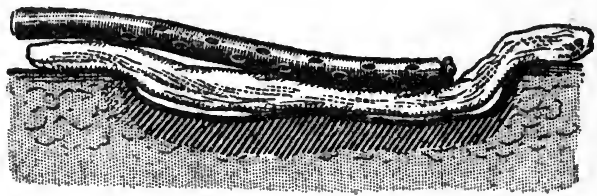

FIG. 47.-Wound with surface horizontal. Wrong method of placing the tube. The perforated instillation tube is on the surface of the compress.

imagined that "wicks" of absorbent cotton would play a similar part. But nothing of the kind occurs. After a short time, the deeper parts of the absorbent tissue become impregnated with the plasma secreted by the tissues and are then almost impermeable to the liquid. Suppose a thin compress be placed on the surface of the wound and a tube be laid on the compress, liquid injected into the tube slips away over the surface of the wound without sterilising the wound (Fig. 47). Therefore it is absolutely necessary to place the tubes directly in contact with the wound-surface, and then to lay the compresses above them (Fig. 48) in such a manner that 


\section{TECHNIQUE OF THE STERILISATION I47}

the liquid may insinuate itself between them and the surface of the wound.

In the disposal of the tubes it is necessary also to take into account the position of the wound. The flow

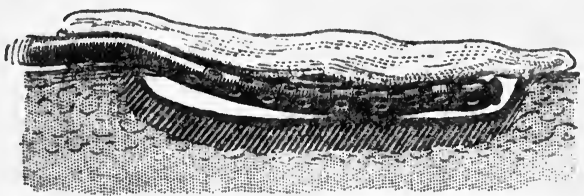

FIG. 48.-Wound with surface horizontal. Right method of placing the tube. Tube in contact with the wound and covered with a gauze compress.

of liquid being under the influence of gravity, the tubes are arranged differently, according as the wound is situate on the anterior, lateral, or posterior surface of the body. They are placed in such a manner that the liquid may spread itself over the greatest possible extent of the wound (Fig. 49). When the wound is on the anterior surface of the trunk or limbs the application of the tubes is easy. If on the lateral or posterior aspect, prolonged contact between antiseptic and wound surface is more difficult to obtain.

The shape of the wound also plays an important part. A wound possessing but a single opening, and that situated superiorly, can be filled with liquid like a cup, and can be readily sterilised (Fig. 50). If a wound of this type has a second opening at the level of its most dependent part, liquid runs through rapidly and the sterilisation is slower. Gravity plays a very considerable part in the distribution of the liquid and the tubes, must be arranged in such a manner as to utilise it.

B. Arrangement of the Tubes according to the shape of the Wound. Ist. Surface Wounds.-One or more tubes 


\section{I48 TREATMENT OF INFECTED WOUNDS}

perforated with minute holes are placed on the wound. If it is situated on the anterior aspect of the body and the bottom of the wound is in the horizontal plane, or nearly so, the liquid can be distributed fairly equally

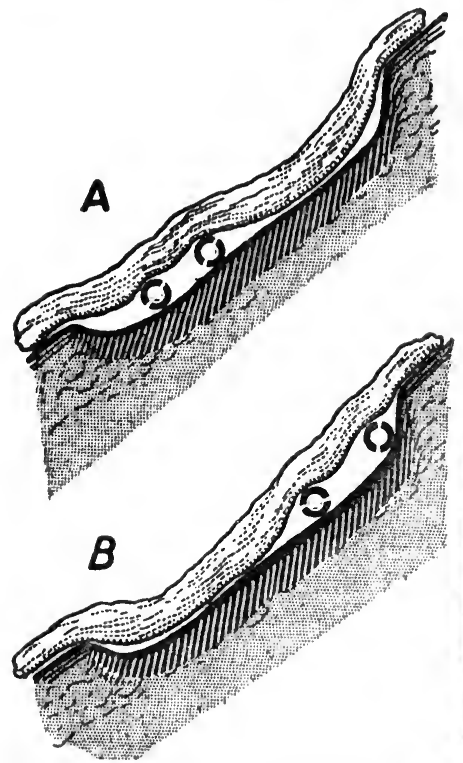

FIG. 49.-Wound with surface inclined.

A. Tubes placed the wrong way, along the lower border of the wound.

B. Tubes placed the right way, along the upper border of the wound.

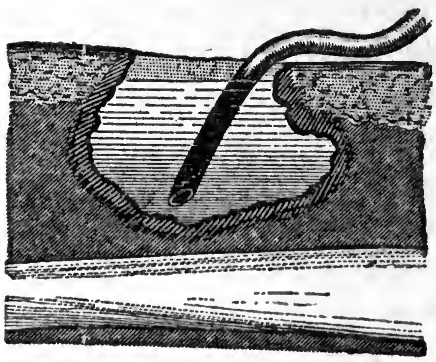

FIG. 50.-Wound with opening superior, so that it can be filled like a cup.

over its surface (Fig. 48). When the surface of the wound is inclined, the tube is laid along the more elevated border (Fig. 49), so that the liquid, carried by gravity, flows over the surface of the tissues. Instead of a simple tube, we may use a ring, formed out of a tube perforated with little holes throughout its middle 
portion, and whose ends are joined by a Y-shaped cannula (Fig. 5I). By means of a thread attached to the two halves of the tube, the loop can be altered to any convenient shape. On the end of a stump, for

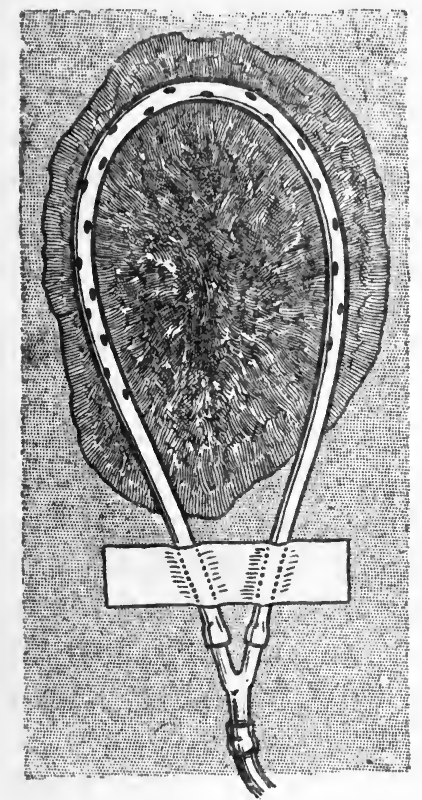

Fig. 51.-Surface wound. The instillation is made by means of a tube perforated in its middle portion, whose ends, fixed to the skin by a strip of adhesive plaster, are joined by a Y-shaped distributor.

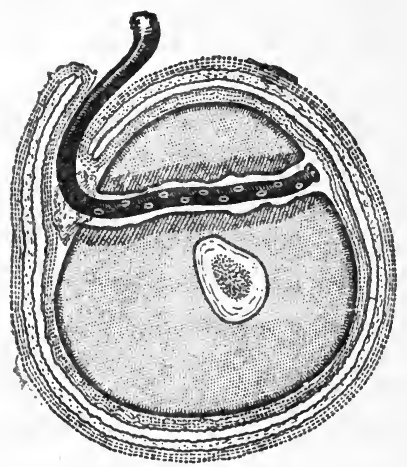

Fig. 52.- "Seton" wound, in the interior of which is placed an instillation tube perforated with small holes and which passes through the dressing at its upper part.

example, this mode of instillation is useful. Between the raw surface and the base of the flap is placed a loop formed of a rubber tube pierced with multiple holes whose two extremities are joined by the $\mathbf{Y}$-cannula resting on the skin of the anterior portion of the limb. 


\section{I50 TREATMENT OF INFECTED WOUNDS}

The fixation of these tubes is effected by means of gauze compresses soaked in Dakin's solution, which are laid over them. In addition, they are fixed to the skin adjoining the wound by a strip of adhesive plaster. This fixation must be thought out very carefully, because if the tubes slip down to the lowest part of the wound, sterilisation of the upper part will be defective. For, whatever precautions may be taken, the tubes sometimes become displaced. That is why it is advantageous, in the treatment of surface wounds, to replace instillation of liquid by the application of chloramine paste, so soon as sphacelated tissues have been dissolved.

2nd. The "Seton" Type of Wounds.--If a tube closed at one end and pierced with small holes is placed in a "seton" wound whose axis is almost horizontal, liquid readily remains in the wound (Fig. 52). But if the axis of the seton is vertical, the liquid escapes by the inferior opening immediately it is injected. Therefore, sometimes, in these cases a tube wrapped in "tissu éponge" is used. This absorbent fabric (Fig. 37) distributes the fluid over the surface of the wound and keeps it there for a period more or less prolonged.

$3 \mathrm{rd}$. Wounds with a Single Orifice.-If the opening is at the "roof" of the wound, the device is simple. A rubber tube bearing a single hole near its blind extremity is introduced to the bottom of the wound (Figs. 50, 53). The cavity of the wound fills up like a cup, and the fluid remains quiescent there until it is displaced by the fresh liquid brought by the tube to the bottom of the wound. The superior opening of the wound should be large enough to allow the liquid to circulate freely. In these cases, "drop by drop" instillation may be used. The 
liquid continually arriving at the bottom of the wound is constantly being renewed. This arrangement is particularly favourable to rapid sterilisation. Therefore, wherever possible, it is well to transform the wounds with two openings into wounds with one opening, by closing the lower aperture with a tampon.

When the opening of the wound, instead of being found on the anterior aspect of the body, appears on the posterior surface, conditions are altered. If the patient can sleep prone on his stomach, the tube is placed as just

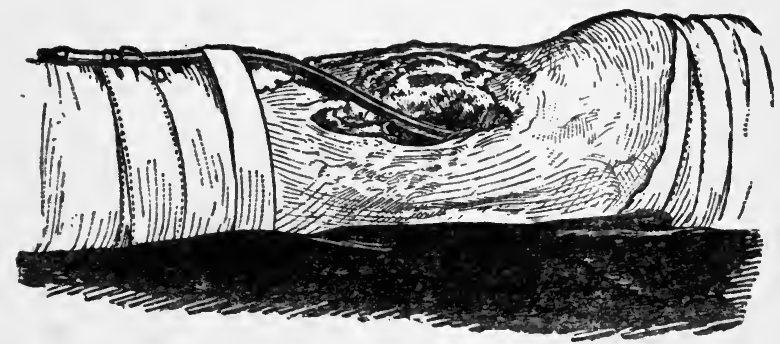

FIG. 53.-Compound fracture of tibia with the opening of the wound on the anterior aspect of the limb; in the seat of fracture is a tube open at the end.

described. Otherwise a different device must be adopted. In fact, if the fluid is led to the roof of the wound by a tube which enters by the lower opening, it tends to fall back immediately, under the influence of gravity.

When the wound is a narrow one, a tube sheathed with "tissu éponge" can be used, which may carry the liquid by capillary attraction to the highest regions (Fig. 54). If the wound is larger, several tubes pierced with little holes are-introduced and the liquid injected under an adequate pressure. The liquid spurts out over 


\section{I52 TREATMENT OF INFECTED WOUNDS}

the walls and succeeds in sterilising them, but more slowly than when it can remain quietly in the wound.

Should the orifice occur on the lateral aspect of the body, a certain amount of retention of the liquid can be attained by compresses plugging the orifice. In this case tubes pierced with small holes and closed at one end are used. In addition, the patient should be placed

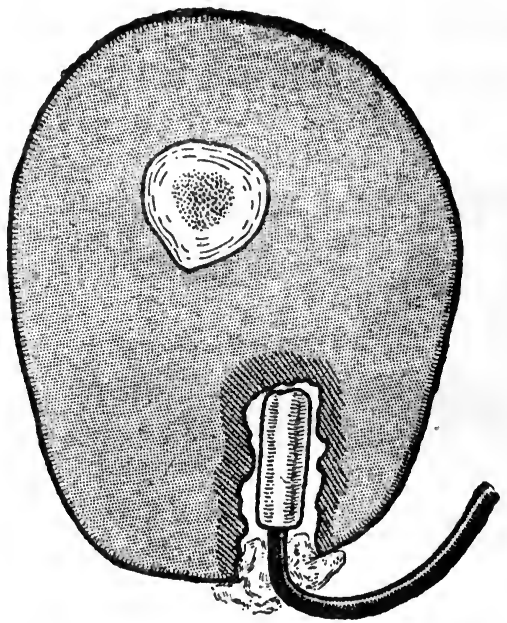

FIG. 54. - Wound of the soft parts whose orifice is at the posterior aspect of the limb. Instillation to the "roof" of the wound by means of a tube sheathed in " bath-towelling" (tissu eponge).

in the position most favourable for retaining liquid in the wound.

4th. Large Wounds with Several Openings.-Sometimes, if the openings are on the anterior surface of the limb, these wounds can be filled with liquid. Sterilisation is then very simple. But in the majority of cases it is not so. The fluid has a tendency to escape rapidly 
by the most dependent point of the wound. In addition to lesions of the soft parts, there is often a fracture which makes the wound still more irregular.

Then tubes perforated over a length of 5 to $10 \mathrm{~cm}$. are used, and introduced as deeply as possible into each diverticulum. To fix these tubes in their positions in

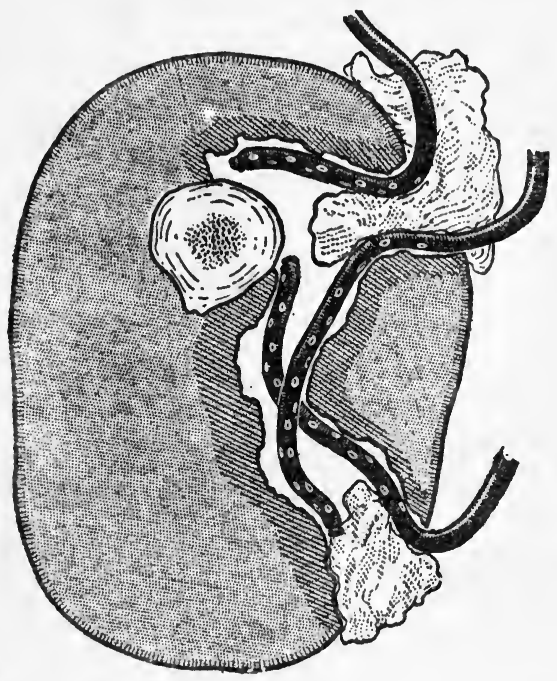

FiG. 55.-Irregular wound of the thigh. Two tubes are placed in the wound anteriorly and one posteriorly. These tubes are applied to the surface of the tissues. They are kept apart by gauze packed between them in the opening of the wound.

the central part of the wound, gauze compresses may be used. But it is important to see that the compresses are not packed too tightly, and that they are always separated from the surface of the tissues by a tube (Fig. 55). Avoid placing tubes in the middle of a mass of gauze (Fig. 56). In fractures of the femur, the wound can be kept open by short pieces of rubber tube $3 \mathrm{~cm}$. (about 


\section{I54 TREATMENT OF INFECTED WOUNDS}

It inches) diameter, which are separated from one another by other pieces of tube placed at right angles. As gravity will not permit the fluid to remain on the surface of the wound, a sufficient number of tubes is arranged so as to moisten every portion of the wound

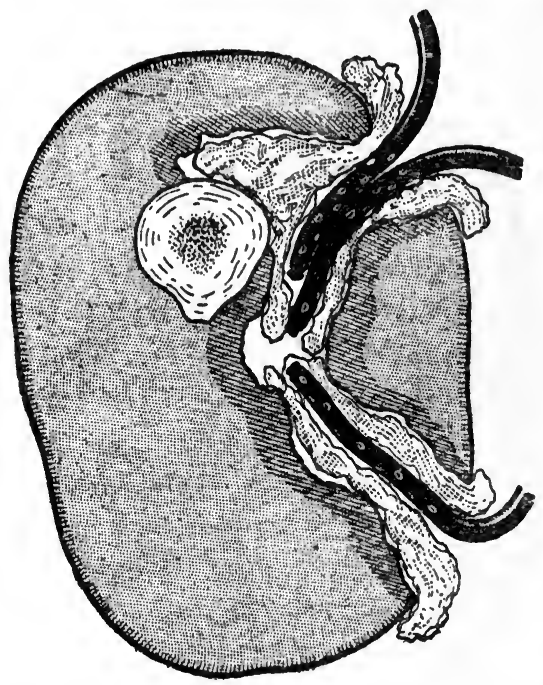

FIG. 56.-The same irregular wound of the thigh. The tubes are wrongly placed. Instead of being in contact with the tissues they are in contact with the gauze which fills the wound.

surface (Fig. 55). In the large wound of a compound fracture of the thigh at least 8 or 10 tubes are needed.

5 th. Wounds of the Brain.-The consistency of the brain prevents the application of simple perforated tubes. The cerebral substance, in fact, presses closely against the walls of the tube and enters the perforations. The liquid no longer circulates properly. In the case of cerebral wounds it is therefore necessary to resort to 
another arrangement, which enables the liquid to circulate in contact with the surface of the wound. The appliance to be used in such cases consists of an external tube, permeable to liquids, and an internal tube of small calibre by which the antiseptic substance is injected. The external tube consists of a very light framework, on which is stretched a thin fabric which has been rendered hydrophilous. This framework Du Nouy constructs of bamboo hollowed and perforated by a thermocautery, while Schmelz and Daufresne make it of thin German silver wire. The diameter of these tubes varies from $I$ to $I^{\prime} 5$ centimetre, and their length from 4 to 6 centimetres. In the interior of the tube is fixed a small rubber tube about $2 \mathrm{~mm}$. in diameter, which is attached to the framework. This little appliance is fixed in the cerebral wound so that the movements of the head cannot displace it. The meninges are protected by a piece of gauze impregnated with vaselin. The appliance is connected with a special apparatus which instils the liquid drop by drop.

C. Arrangement of the Tubes according to the State of Infection. Ist. Fresh Wounds.-Fresh wounds nearly always bleed. If tubes pierced with small holes be placed in a wound containing fresh blood, the tube will be filled with it, the blood will coagulate, and the lumen of the tube will be obliterated. It is essential, in fresh wounds, to arrest haemorrhage thoroughly, before arranging the tubes, and to verify their permeability with care, before continuing the dressing. Fresh wounds having no secretion, or very little, tubes sheathed in absorbent fabric may be applied to their surface without inconvenience. For the same reason, gauze is less harmful 


\section{I56 TREATMENT OF INFECTED WOUNDS}

on fresh wounds than on wounds which are suppurating.

2nd. Suppurating Wounds.-The presence of pus on a wound is an indication that tubes surrounded by absorbent tissue may not be used, because this fabric immediately becomes saturated with pus. For the same reason "wicks" and gauze compresses are used as little as possible, and tubes multiplied. Gauze may be used at the orifice of the wound. But all the diverticula should

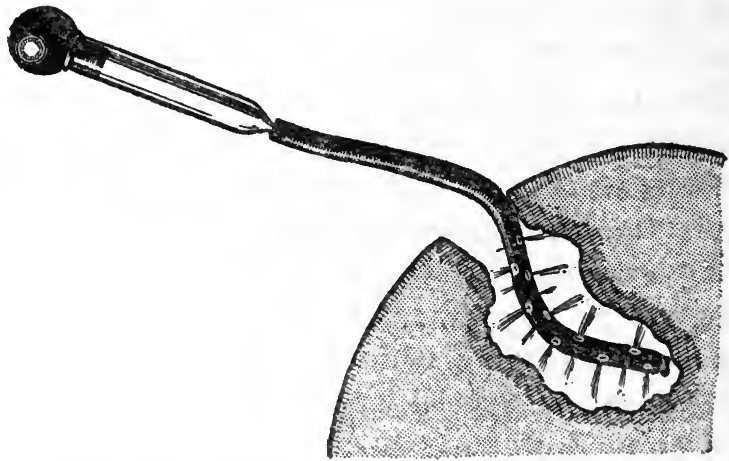

Fig. 57.-'Testing the permeability of a conducting tube at the time of dressing.

contain tubes and not gauze. It is advisable to have the tubes more numerous than in a fresh wound of the same dimensions.

D. Testing the Working of the Tubes.-Before the dressing is applied, the permeability of the tubes and their perforations should be tested (Fig. 57), also the manner in which the various regions of the wound are receiving their share of the antiseptic liquid. This test is to prove that the tubes have not become plugged with blood-clot, and that the distribution is taking place 
evenly over the whole surface. Further, it shows what quantity of liquid will be needed to fill the wound completely, or to moisten the entire surface, should its position not allow of its being filled.

The nurse should be present at this testing, which will also show her how to control the flow of liquid in the wound without wetting the patient.

\section{DRESSING}

Ist. Method of carrying out the Dressing.--As soon as the tubes are in position, gauze compresses soaked in

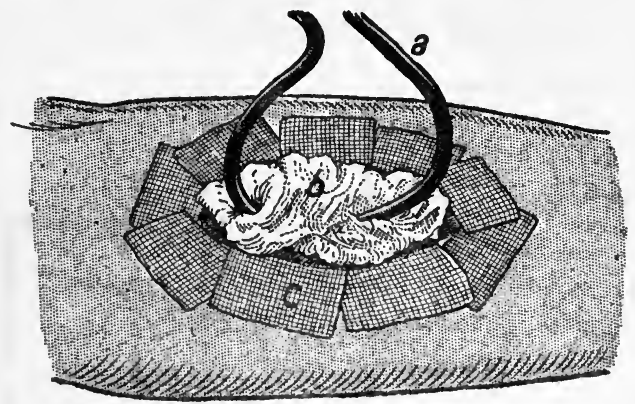

FIG. 58.-Dressing : $a$, Conducting tube kept in the wound by gauze placed in the orifice; $b$, Squares of gauze sterilised in vaselin placed on the skin around the wound.

Dakin's solution are applied. These compresses help to fix the tubes on the surface of the wound. The tubes have been selected long enough to allow several centimetres of their non-perforated portion to be outside the dressing (Fig. 58). Also the perforated part must be buried wholly in the wound, because otherwise the free openings would allow fluid to escape unused, possibly doing harm. 
After the application of the compresses to the wound, the adjoining skin is protected by squares of gauze, sterilised in vaselin (Fig. 58). Pieces 8 or $10 \mathrm{~cm}$. square are placed in yellow vaselin and sterilised. At the moment of dressing, they are taken up with dressing forceps and applied to the surface of the skin, to which they immediately adhere. They form an excellent protection for the skin, which, on the posterior aspect of the trunk or limbs, has a tendency to become irritated by the hypochlorite.
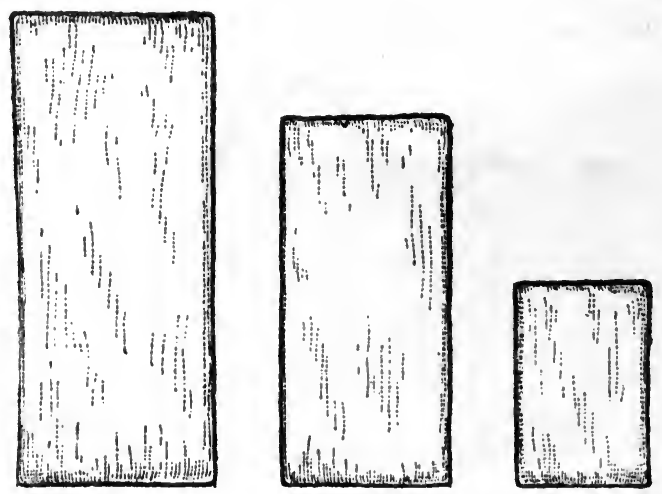

FIG. 59.-Sheets of dressings, composed of layers of absorbent cotton-wool, non-absorbent cotton-wool, and gauze.

The dressing is completed by a sheet of cotton-wool protected on either surface by one thickness of gauze. This dressing is prepared beforehand in three different sizes (Fig. 59). It is composed of four strata ; a layer of gauze, a sheet of absorbent cotton-wool, a sheet of non-absorbent cotton-wool (coton carde), and a final cover of gauze (Fig. 60). The side which has the absorbent cotton-wool is applied next the wound. 


\section{TECHNIQUE OF THE STERILISATION I 59}

Secretions are thus absorbed, without being able to escape readily to the exterior, by reason of the presence of the non-absorbent cotton-wool. At the same time, evaporation goes on quite easily through this almost

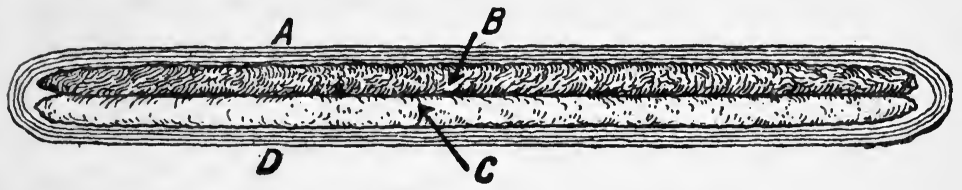

FIG. 60.-Section of the sheet of dressing : A, Gauze. B, Carded (nonabsorbent) cotton-wool. C, Absorbent cotton-wool. D, Gauze.

waterproof layer. Waterproot fabrics should never be used.

The application of the dressing is speedy. The middle part of the dressing is placed under the limb and

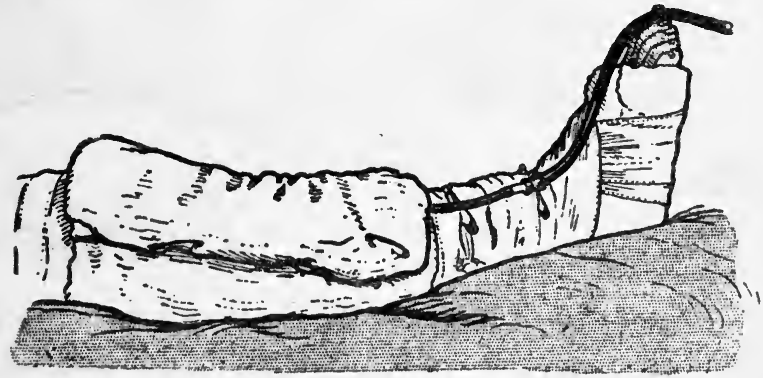

FIG. 6r.-Dressing applied around a compound fracture of the leg, and fastened by safety-pins; the distributing tube is fixed to the plaster apparatus by safety-pins.

the two sides are fastened on the anterior surface of the limb by two or three safety-pins. The use of a bandage is thus avoided. Besides, the dressing is easily undone, and the wound can be examined and the position of the tubes ascertained without disturbing and distressing the 
patient. When the dressing is first applied, two scissorcuts are made in the layer of cotton-wool to allow the rubber tubes to emerge readily from the dressing (Figs. 62 and 63).

2nd. Fixation of Tubes and Cannulae.-When the dressing is finished, the ends of the supply-tubes emerge at different points from the layer of cotton-wool and gauze. These tubes are connected up in groups of two or four by means of the branched unions or cannulae

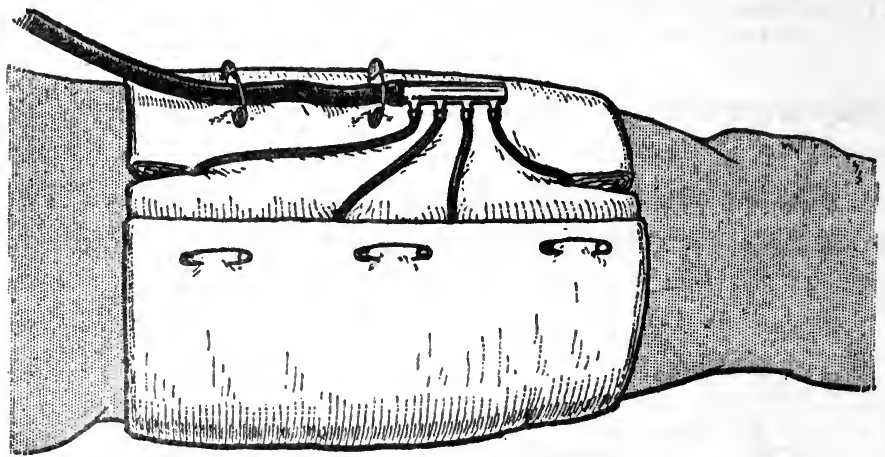

FIG. 62.-Position of the distributing tube on the surface of the dressing. The conducting tubes penetrate the dressing, either at the point where the end of the layer of cotton-wool and gauze overlaps, or through windows cut with scissors.

which have been described (Figs. 62 and 38). In the case of a compound fracture of the thigh, the eight tubes are divided into two groups and united by two cannulae of four branches each (Fig. 63). In the case of a very extensive wound where certain of the small conducting tubes are too short to be connected up with the branches of the cannula, they are lengthened by pieces of rubber tube and "unions" or connecting tubes of glass (Fig. 39). This work can be done after the dressing, when the irrigating apparatus is installed. 
After the tubes have been joined up to the cannula, this latter is fixed to the highest part of the dressing. For example, in a compound fracture of the thigh, the cannula is fixed above the middle of the anterior aspect of the limb. This fixing is simply done by nipping the

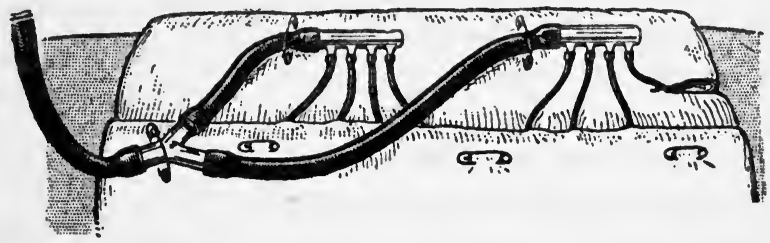

Fig. 63.-Arrangement on the surface of a dressing of a $Y$-connecting tube, and of two distributing tubes with four branches.

largest part of the glass cannula in a big safety-pin, itself attached to the dressing. Then the larger end of the cannula is united to the irrigating tube which is attached to the flask or other reservoir of liquid. The correct

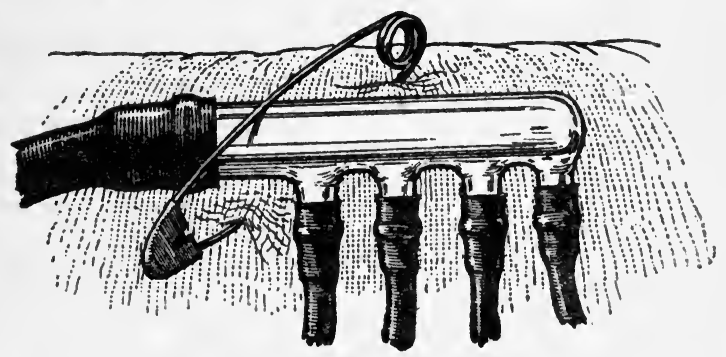

FIG. 64.-Method of fixing a distributing tube to the surface of a dressing.

fixing of the cannula to the surface of the dressing is important. Thanks to it, the small conducting tubes lie in the wound, in the positions in which they have been placed, without either the weight of the irrigating tube or the movements of the patient being able to shift them. 


\section{I62 TREATMENT OF INFECTED WOUNDS}

3 rd. Immobilisation of the Limb.-Naturally the limb should be prevented as much as possible from moving. Either plaster apparatus, suspension, or continuous traction will be used. In every case where it is indicated, the patient is placed on a Bradford's frame. When the time for dressing comes, the frame is raised, and one or two bands are removed, so that the posterior portion of the limb or trunk can be examined or dressed without moving the patient.

The dressing is renewed every twenty-four hours. If, however, before the expiration of this period, the cottonwool has become very wet, the outer layer of the dressing may be changed without disturbing the tubes or the layer of gauze which covers the wound. The changing of the dressing consists in removing the gauze compresses which are on the surface of the wound, and at the entrance to it. The position of the tubes is carefully checked, and modified if there should be need. No washing is done, simply fresh gauze and an external dressing applied. The manipulations are thus extremely simple, and, in a short time, the surgeon can personally dress a large number of cases.

The mattress is protected by a waterproof sheet. The quantity of liquid used should be always so small that the bed is not flooded.

\section{Instillation of the Antiseptic Liquid}

The flask holding a litre, or other convenient reservoir, is filled with Dakin's solution, coloured to a rosetint with permanganate of potassium. This coloration distinguishes Dakin's solution from physiological saline solution, and most assuredly prevents mistakes. 


\section{TECHNIQUE OF THE STERILISATION I63}

Ist. Continuous instillation gives better results than intermittent instillation. But it is not so frequently employed. In fact, it is only suited to wounds where the liquid can remain in quantity, or to small wounds for which a single conducting tube sheathed with absorbent fabric will suffice. The flow of the liquid is regulated by means of a screw pinch-cock interposed between the flask and the drop-counter. Five or six drops per minute will usually give sufficient moisture to this type of wound. It should be remembered that the pressure of the liquid at the surface of the wound is represented by the difference in level between the wound and the lower portion of the dropcounter, and not between the wound and the upper portion of the reservoir. If the drop-counter be placed too low, on a level with the wound, it will not work. It is equally necessary to be aware that drop-by-drop instillation should only be used when the end of the irrigating tube is connected up with only one of the little tubes which distribute liquid to the wound. Under these conditions, continuous instillation permits the degree of concentration of the antiseptic liquid on the surface of the wound to be maintained under better conditions than intermittent instillation.

Intermittent instillation is used for the greater number of wounds. As a matter of fact, the great majority of wounds are extensive and irregular and have several openings. To these continuous instillation is not suited.

Intermittent instillation is carried out by releasing for a few seconds, every two hours, the pinch-cock which is placed on the irrigating tube just below the reservoir. 


\section{I64 TREATMENT OF INFECTED WOUNDS}

Liquid immediately escapes from the flask (irrigating bottle or reservoir), and spurts out in great abundance from every hole of all the conducting tubes. The duration of flow of the liquid should be very short, lest the patient be flooded out. The quantity thus injected varies, according to the nature of the case, from 20 to 100 c.c. $^{1}$ and sometimes more. As a general rule, the injections are made every two hours; occasionally, with greater frequency. When the apparatus is installed as we have described, the work of the nurse in charge of the instillations is very light. In fact, as in each case she halts at the foot of the bed, she has only to press for a few seconds the spring "pince de Mohr" fixed on the irrigating tube.

Intermittent instillation is practised every two hours, but it would be of advantage to practise it more frequently, as the hypochlorite decomposes very rapidly when in contact with the tissues. On the other hand, more frequent instillations often make too great a call on the energies of the nurses. In order to diminish the amount of manual work required, and, where desirable, to increase the number of instillations, attempts have been made in the direction of automatic instillation. The automatic devices which have hitherto been employed are based on the principle of the intermittent fountain. As a rule, they work well under experimental conditions, but in the actual conditions of surgical treatment they reveal defects. In the case of small discharges more especially, the siphon fails to prime, and the liquid runs drop by drop into the tube, or the siphon does not discharge itself, and again the liquid flows drop

$$
1 \text { Say from oz. to } 3 \frac{1}{2} \mathrm{oz} \text {. }
$$




\section{TECHNIQUE OF THE STERILISATION 165}

by drop. It has therefore been necessary to abandon apparatus based on this principle, as its working was very irregular, and required almost as much attention as the ordinary apparatus.

As a result of experiments carried out by Lecomte du Nouy in the Compiègne laboratories, an electromagnetic distributing apparatus for the irrigation of wounds has been invented. It consists of an individual electro-magnetic distributor and a clockwork device for making contact. The electro-magnetic distributor consists of the bobbin of an electric bell, a core, an armature, the latter movable, and a wooden stopper, which contains the whole, and is fixed upon the neck of the ampoule. The current, automatically transmitted, raises a valve which closes the bottom of the ampoule, and the liquid flows until the current ceases. The principle consists in the utilisation of the well-known solenoid coil, through which is"sent, for a very short time, a current of sufficient strength to produce a mechanical effect. The bobbin, which could not resist such a current if long continued, can easily carry it for a few seconds. Calculation shows, in fact, that the instantaneous rise of temperature - taking into account the fact that radiation is practically nil-is, for a bobbin of 9 ohms resistance, covered with copper wire $0.4 \mathrm{~mm}$. in diameter, $123^{\circ} \mathrm{C}$. per second for a current of 2 amperes. This current seems at first sight to be a considerable load for such fine wire, but as it flows through the bobbins for one or two seconds only, it does not overload them, nor does it entail any appreciable expense. In fact, a group of 12 such appliances, served by a current of 220 volts pressure, consumes barely 0.15 kilowatt per month, so that it costs only a 


\section{I66 TREATMENT OF INFECTED WOUNDS}

shilling or so yearly. Contact is established every halfhour by means of a clock or clockwork train, and the current is sent through all the appliances, mounted in series, the number of the groups in series depending on the tension of the current and the resistance of the bobbins. It is important that the contacts shall be of regular duration, and the contact-making device must be reliable in working. Daufresne has worked out an arrangement of this kind by means of a very simple alteration of a striking clock. One clock suffices to control any number of such appliances-enough, for example, to serve 500 or 1000 beds.

Each time contact is made by the clock the valves of the ampoules are lifted for two seconds, and the liquid flows. The quantity of the liquid reaching the wound may be regulated by reducing as required the calibre of the supply-tube. As the frequency of the irrigations does not increase the work of the staff, and as it is of advantage that the liquid in the wounds should be renewed as often as possible, instillations are now practised every half-hour.

2nd. The total quantity of liquid injected in 24 hours varies from about 250 to 1200 c.c. ${ }^{1}$ In very extensive wounds, more can be injected without inconvenience. The only fixed rule is, that the wound should be kept constantly moistened by the liquid, without the patient being made uncomfortably damp.

3rd. In intermittent instillation, the pressure varies from forty centimetres to a metre. It should be regulated according to the particular needs of the wound and the sensitiveness of the patient. At the moment of

1 Roughly, eight ounces to two pints. 


\section{TECHNIQUE OF THE STERILISATION 167}

commencing the instillation, he experiences sometimes a slight impression of pain which may last some minutes. Sometimes, again, he has only a sensation of chilliness, or actual cold. The patient should never suffer actual pain from the instillation. Should he complain, it shows that an error of technique has been committed. The pain may be due to excess of pressure, or to the woundopening being too small. If the pressure be too great, the liquid spurts out violently from the apertures in the tubes against the walls of the wounds and bruises the tissues. That is why the pressure should never be greater than one metre. With sensitive patients, a pressure of 20 to 30 centimetres is sufficient. Another cause of pain is retention of the liquid in the wound under pressure. If the incisions are too limited, and if the conducting tubes are too tightly gripped by the tissues or by compresses, the liquid cannot escape freely from the wound. It accumulates under pressure, and the patient feels it. The wound should be freely opened up, so that the liquid may escape without hindrance.

\section{DuRation of the Instillation}

Instillation of liquid continues day and night until all microbes have disappeared from the "smears." Therefore it is inspection of the microbial curves which indicates when the irrigation can be stopped. So long as a few microbes remain, no alteration should be made either in the quantity of the liquid or in the frequency of the instillations. So long as a focus of infection, be it ever so small, remain on the surface of the wound, total reinfection is possible. If the instillations be stopped, or 


\section{I68 TREATMENT OF INFECTED WOUNDS}

their frequency lessened, when the microbial curve shows only one or two microbes per field of the microscope, rapid reinfection may be brought about. On the other hand, the presence of hypochlorite does not lessen the rapidity of repair. By suppressing microbes, it accelerates it. As the few small infected foci which still persist on the wound after some days of instillation cannot enlarge, the greater part of the wound cicatris $s$ with the same speed as if it were aseptic.

In general, from three to ten days are needed to sterilise a wound of the soft parts, and fifteen days or more a compound fracture. These figures are those observed when the wound is sterilised before the suppurative stage. But if the treatment is commenced after the wound has already suppurated, the duration of the instillation period is usually much longer. Bacteriological examination alone can indicate the time when the instillations may be discontinued.

\section{ERRORS OF TECHNIQUE}

A. Insufficient Penetration of the Liquid.-Whenever examination of the curve of sterilisation shows that, before attaining surgical asepsis, the line has become horizontal, we may be sure that a fault in technique has been committed. We know, in fact, that the diminution in the number of microbes in a wound should progress steadily, whenever the antiseptic liquid is carried into all the regions infected. If sterilisation is not achieved, in the first place it is necessary to ascertain that the Dakin's solution contains the needful amount of hypochlorite, 
and afterwards to look into the possible causes which could hinder the penetration of the liquid throughout the wound. The causes are generally as follows:-

Ist. The distribution of the liquid in the wound has not been completely accomplished, by reason of : (a) slipping or detachment of one of the conducting tubes ; $(b)$ obliteration of the lumen of a tube by bloodclot; $(c)$ kinking in a tube, due to faulty placing; $(d)$ the omission to put a conducting tube in some diverticulum of the wound. Should a tube be placed in a passage too narrow which it fits tightly, there can be no return flow of liquid between the wall of the tube and that of the wound, and, in consequence, no instillation. Careful examination of the wound will enable us to ascertain the presence of one or more of these causes of error.

2nd. There is some error in the installation of the irrigating apparatus. The fault most frequently committed is that of putting a drop-counting appliance in communication with several tubes. As the output is very small, the liquid, obeying the dictates of gravity, runs down one of the tubes while nothing goes to the rest. The same thing may happen in intermittent irrigation, if the calibre of the principal tube or the inferior orifice of the flask (reservoir) is too narrow. In this case the outflow is insignificant, and instead of the liquid being distributed to four or eight tubes, it passes along only a few of them, and, in consequence, a whole region of the wound is deprived of liquid. This mistake will be avoided if the instructions we have already given on the subject of the relative calibres of the different tubes and the installation of the irrigation apparatus be followed precisely. 


\section{I7O TREATMENT OF INFECTED WOUNDS}

3 rd. The quantity of liquid is insufficient. Inadequate instillation is most frequently seen, when, instead of using irrigating apparatus, a syringe is employed. As the tubes are multiple, the nurse has to spend much time in injecting the needed amount with a syringe. Therefore, whenever this method is in use, the quantity of antiseptic is frequently found to be insufficient. The same thing happens in using irrigation apparatus, when, through negligence, the irrigations are omitted, or made at too long intervals during the night. Likewise when a tube passed into too narrow a track blocks up its lumen, so that no circulation is established (Fig. 65). By carefully examining a wound we find indications which lead us to suspect the insufficiency in quantity of liquid. Two symptoms present themselves in these cases. One is, the pus beginning to have an unpleasant odour, for a well-irrigated wound should be perfectly inodorous. The second is absence of the characteristic changes in the secretions. The discharge from a well-irrigated wound should be thicker and more transparent than the normal secretion. The presence of unmodified secretions in a wound permits one to assert, either that the liquid does not contain the sufficient amount of hypochlorite, or that the instillation is not being carried out in the prescribed manner.

B. Excessive Quantity of Liquid.-When the liquid is allowed to flow too long over the surface of a wound, or in quantity too abundant, the absorbent cottonwool of the dressing, and evaporation, are not equal to the task of getting rid of the excess of fluid. The bed becomes flooded, the limb bathed in Dakin's solution, and the skin becomes irritated. An excessive 


\section{TECHNIQUE OF THE STERILISATION I7I}

quantity of liquid has no deleterious action on the wound, but it worries the patient. $\mathrm{He}$ is in an uncomfortable plight, and ulceration of the skin, more or less painful, may be produced. Therefore the nurse must learn how to regulate the quantity of liquid so that the

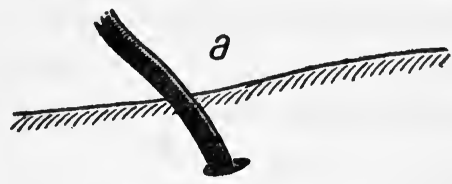

$\rightarrow$
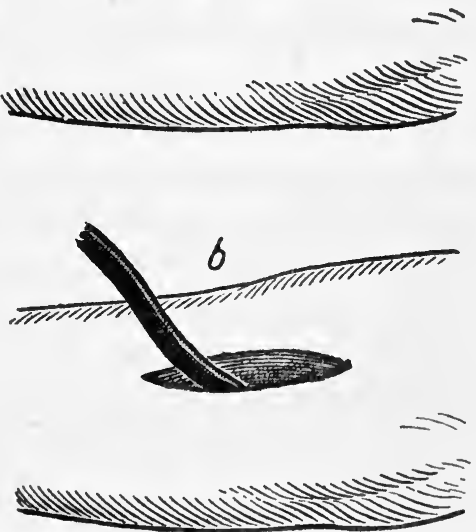

FIG. 65.-Relative dimensions of the orifice of the wound and of the conducting tube. a, Faulty arrangement.-The opening is much too small, the liquid is under pressure in the $\operatorname{limb}$, and its circulation is impossible. $b$, Correct arrangement. - The opening is large enough to allow the free return of the liquid between the wall of the wound and the outside of the tube.

wounds are sufficiently moistened without the patient being made damp. With a little attention nurses soon avoid injecting too much liquid into the wound. Besides, it is always better to use too much than too little, for the inconvenient results of too much liquid are not 


\section{TREATMENT OF INFECTED WOUNDS}

serious and can be remedied easily. By applying carefully squares of vaselined gauze (p. 158) to the skin about the wound, it can be protected completely against the lesions produced by an excess of liquid.

C. Excessive Pressure.-We have noticed already that an excess of pressure may be due to two quite different causes - a too great elevation of the reservoir of fluid above the level of the bed, or to smallness of the incision which hinders a ready reflux of the liquid between the walls of the wound and the conducting tube (Fig. 65). Excessive pressure of liquid in the wound brings about distress. The moment instillation causes a patient pain it must be discontinued, and the error of technique which is the cause of the pain must be discovered. 


\section{CHAPTER $V$}

THE CLINICAL AND BACTERIOLOGICAL EXAMINATION OF WOUNDS

EVERY infected wound should respond to chemiotherapy, when this is applied in correct manner. It is necessary, therefore, that the progress of treatment should be controlled each day by examination of the wound, and that the technique should be modified according to the results of this examination. Clinical and bacteriological study of the wounded patient, and of the wound, is the indispensable guide in therapeusis.

\section{Clinical Examination}

The aspect presented by wounds is modified under the influence of treatment in a manner more or less rapid according to the nature and age of the lesion. This evolution varies according to the period of infection during which sterilisation was commenced.

A. Modifications of the Local Conditions. Ist. Fresh Wounds.-Immediately after the infliction of the injury, blood pours out between the edges of the wound and forms a clot. Up to the sixth or twelfth hour, there is not, as a general rule, either swelling of the tissues or secretion 


\section{I74 TREATMENT OF INFECTED WOUNDS}

on the surface. At the same time, we have sometimes met with wounds only six hours old containing gas and giving off a putrefactive odour. Towards the twentyfourth hour wounds secrete slightly. When instillation is begun between the sixth and the twelfth hour, the tissues retain their normal appearance. Muscles remain red and cellular tissue is not changed. If the tissues have been severely bruised they necrose, but neither redness nor swelling is seen at the margin of the wound. At the end of three or four days the necrosed tissue becomes of whitish colour and soft consistence. It begins to become detached in fragments from the deep parts. Red portions begin to show themselves. Towards the eighth day following the injury, the wound is usually clean. The surface is of a bright red. Secretions are almost nil. The margins of the wound are not swollen and present no evidence of lymphangitis. Should signs of inflammation appear, it is certain that a fault in technique has been committed, either in the manufacture of the liquid, or the disposition of the instillation tubes. Towards the tenth day, the entire surface of the wound is even and red. In the most irregular portions, and by the lymphatics of vasculonervous bundles, sometimes a few drops of pus may be seen. The limb has regained its normal size. The integuments about the wound are supple and not tender on pressure. The skin is not yet adherent to the deep parts. That is the reason why, wherever possible, wounds should be closed before the twelfth day.

The integuments are sometimes modified, after the lapse of a few days, by the application of Dakin's solution. They become red and painful. This complication 
may be due to one of several causes. The tincture of iodine which has already irritated the skin is generally the cause. But the Dakin's solution may have been badly made. If Dakin's solution contains too much alkali, it becomes as dangerous as eau de Javel or Labarraque's liquor. The moment irritation of the skin occurs, the solution should be examined to see if it fulfils the conditions laid down by Dakin. It sometimes happens that a solution perfectly prepared may cause redness in subjects who have an exceptionally delicate skin, or when the wound occupies the posterior aspect of the trunk, the pelvis, or the limbs ; or when the dressings are too tightly applied, or changed too infrequently. The best way to avoid irritation of the integuments about a wound is to cover the skin with squares of gauze sterilised in yellow vaselin. If the wound is on a limb, it is useful to employ American suspension apparatus. If the trunk or pelvis be affected, the patient should be placed bodily upon a Bradford's frame. Irritation of the skin due to Dakin's solution is very rare, and is easily distinguished from the lymphangitis so frequent in wounds treated aseptically.

Towards the twelfth day, granulations begin to cover the wound at the same time as the epithelial margin develops. The skin becomes adherent to the subjacent parts. The whole surface of the wound is composed of rose-tinted granulations. Cicatrisation comes about in a regular manner, without any interval of retrogression, such as one is accustomed to in wounds treated by the aseptic method. The cicatrisation curve develops symmetrically, following the algebraic formula of Lecomte du Nouy. 


\section{I76 TREATMENT OF INFECTED WOUNDS}

The secretions of wounds thus treated are not very abundant, especially when pains have been taken carefully to resect contused tissues. At the beginning, the compresses are covered with a thick greyish secretion, resulting from the combination of pus and hypochlorite. Then, little by little, the secretion becomes more sticky, clearer, and at last, colourless. At this stage, it is probable that sterilisation has been attained.

2nd. Gangrenous and Phlegmonous Wounds.-When wounds have reached the stage of inflammation by the time the treatment is commenced, the clinical modifications which they undergo under the influence of sterilisation are less rapid. If the liquid can reach all the infected regions, redness, swelling, and pain diminish at the end of one or two days. But if the lesions cannot be reached, even at the price of free incisions, results of treatment are negative. In a general way, when tubes have been placed in all the infected regions, the wound takes on the appearance previously described at the end of a few days. When the tubes have not been able to reach all the infected regions, but when a great portion of the wound has become sterile under the influence of the treatment, the septic regions situate beyond the reach of the liquid accelerate their spontaneous disinfection. It would appear that, the volume of infection being lessened, the organism defends itself more readily.

In all the cases where incisions facilitate the penetration of the antiseptic into gangrenous foci, gas and odour are the first to disappear, then the necrosed tissues dissolve. They are eliminated after the lapse of a few days, without the margins of the wound presenting any inflammatory reaction. 
It is important to notice the rapid disappearance of pain in these cases of infected wounds. As soon as Dakin's solution has got rid of the infiltration of the tissues, the dressings cease to be painful. Wounded men whose wounds are sterile do not suffer.

3 rd. Suppurating Wounds. - In wounds of long standing, which are already freely suppurating when the antiseptic treatment is begun, the earliest sign of the action of the antiseptic is a characteristic change in the pus. This takes on a viscous consistency, while its colour becomes yellowish, transparent. In a few days it lessens in quantity, then disappears. Granulations change their aspect and become red and even. If, the technique being correct, these modifications do not present themselves, it is certain that in the depths of the wound there exists a foreign body.

In wounds of the soft parts, suppuration disappears completely at the end of two or three days. A little thick transparent liquid still remains on the surface of the wound after it has become surgically sterile. In compound fractures, suppuration continues so long as the liquid is not introduced into all the cavities where microbes are found. If suppuration remains stationary, it is certain that there is a sequestrum, or an infundibulum where the liquid is not penetrating. Without further delay, the necrosed splinters should be removed, and the wounds placed under conditions which will allow the liquid to penetrate everywhere.

B. Modifications of the General Condition. - At the outset of the evolution of fresh shell and bomb wounds, fever persists for several days. Frequently, beginning at the third or fourth day, the temperature drops, little by 


\section{TREATMENT OF INFECTED WOUNDS}

little ; sometimes, in deep irregular wounds, it may keep up longer. When the tubes are well placed and the instillation of the antiseptic is adequate over the whole surface of the wound, a dissociation or want of relation between the temperature and the other signs of infection is produced. Often cases are seen with an elevated temperature, but without the general signs of intoxication. They eat and sleep in almost normal fashion. The tongue is pink and moist. They are calm, complain of no pain, and do not look like sick men. This condition may be attributed to the destruction by the hypochlorite of the substances which produce the general symptoms of infection, or to a considerable diminution in the volume of infection. In these cases the infection manifests itself only in the high temperature.

The persistence of pyrexia amongst cases whose wounds are in a fair way of sterilisation is due, generally, to the presence of a small diverticulum where the liquid is not penetrating. In fresh compound fractures the wound surface may be protected against the antiseptic by necrosed tissue, by a compress, or by a blood-clot. As a consequence, infection develops and persists in the region which is in this manner withdrawn from the action of the antiseptic. It may happen also that the tubes are not placed deep enough, or that the liquid is not distributed over the whole surface of the wound. Almost the whole of the wound is sterilised, but at the point not irrigated infection continues. But, usually, this infection is too slight to give the patient the appearance of a sick man. There is a profound difference between the facial appearance of a patient whose wounds are in a fair way for sterilisation, even if he still has some fever, and the 
"look" of a man whose wounds, treated aseptically, are still suppurating. In suppurating cases, even when the wounds are well drained and the temperature but slightly raised, frequently the general signs of septic intoxication are found. These men do not sleep. Appetite is gone and the tongue is dirty. They are at the same time agitated and depressed, and they are in pain. The complexion is leaden. In a word, they are sick men. Immediately these cases are treated by the antiseptic method and suppuration begins to lessen, the general condition changes. After a short time they take on the appearance of cases whose wounds are sterile.

Very rarely, there are cases in which septicaemia develops at the same time as the wound is becoming sterile. We have seen a case die of staphylococcal septicaemia, while the fractured thigh from which he suffered was in excellent condition. Staphylococci had invaded the circulation before sterilisation had had time to become effectual. But, happily, experience has shown that septicaemia is exceptional when the cases are suitably treated.

C. Value of Clinical Observation.-Clinical observation allows one to presume what may be the state of the wound, but it yields no certainty. In fact, wounds whose margins present neither oedema nor redness, whose surface is covered with even granulations and whose secretion is of the slightest, may still be strongly infected. The following case is an example of this. After section of the deep femoral by a shell-wound, a free incision had exposed the sheath of the sciatic nerve, which was filled with blood. After a few days this extensive wound had an excellent appearance. The man 


\section{I80 TREATMENT OF INFECTED WOUNDS}

was in no pain, and had no pyrexia. A little lemoncoloured serum flowed from the wound. It was collected in a pipette. But the general appearance of the wound was so favourable that it was closed with strapping, without waiting for the results of the bacteriological examination. That evening the case had a temperature of $40^{\circ} \mathrm{C}$. (nearly $104^{\circ} \mathrm{Fahr}$.), and the wound had to be taken down. The surgeon then asked for the bacteriological report, and learned that the transparent liquid contained chains of streptococci. Hence in certain cases clinical observation is absolutely impotent to instruct us as to the real condition of a wound.

Wounds also are met with, covered with greyish granulations and with a puriform liquid, which are aseptic, and which may be sutured with success.

Clinical observation should be looked upon as an adjunct to the bacteriological examination. Wounds identical in appearance, from the clinical point of view, may be in very different microbial conditions. Between a wound which yields five or six microbes per field of the microscope, and a wound which contains none, usually there is no appreciable clinical difference. All the same, the few microbes which remain on the surface of the first wound can retard by one-half the rapidity of its cicatrisation. The presence of these microbes is important, for it prevents our suturing. Hence the aid of the laboratory is needed constantly to ascertain the progress of sterilisation. 


\section{BACteriological Examination}

The object of the bacteriological examination is to demonstrate the progress of sterilisation and to mark the moment at which this sterilisation is advanced sufficiently to allow of effectual closing of the wound. It is necessary that the quantity of microbes contained in the wound should be known. Since wounds should be examined every two or three days, and as in most hospitals there is no bacteriological specialist, the technique has been made so simple that a large number of examinations can be made by those possessing little experience in bacteriology. The secretions of the wounds are studied by means of "smears." This summary proceeding allows certain qualitative reports to be made, but, more important, it allows of an approximate enumeration of the microbes contained in the secretions. Thanks to it, the diminution in the numbers of the microbes can be made known as the treatment progresses, up to the date of their total disappearance. We have determined empirically that the disappearance of microbes from the smears indicates a degree of asepsis compatible with closure of the wound. In spite of its crudeness, this method is to be preferred to the usual procedure of bacteriology. In truth, "smears" show what the wound contains, while cultures indicate what may grow under certain conditions. Cultures must be relied upon if it is desired to learn if a wound is bacteriologically sterile, or when it is important to know not only the volume but the nature of the infection. The culture method may also be used in that stage of 


\section{I82 TREATMENT OF INFECTED WOUNDS}

infection in which smears do not give reliable information, that is to say, during the first twelve hours. At this period, in fact, microbes are in such small numbers, and so diluted by the blood, that they cannot be seen in the smears.

A. Technique. Ist. Method of taking Specimens of the Secretions.-During the first six or twelve hours secretions are absent from the wound. The walls bleed more or less freely, and smears of blood taken from the wound show no microbes. Specimens should be taken from the parts of the wound which are not bleeding, in the neighbourhood of, or from the surface of, shreds of clothing or shell splinters.

Wounds older than twelve hours usually have some secretion. As the haemorrhage is arrested, secretions can be taken easily from a region where the secretions are not diluted by blood. Always the points chosen are in contact with shreds of clothing or bits of shell, for in these regions the primary infection is to be found at its maximum.

The specimen is taken by means of a rigid platinum wire mounted on the end of a glass rod (Fig. 66). Should the wound be undergoing continuous instillation, the treatment must be interrupted for two hours at least, before the time when the specimen is to be taken, in order that the secretions may not be diluted by hypochlorite. The tubes are withdrawn and the compresses removed with the greatest gentleness, in order not to provoke haemorrhage. The spot from which the specimen is to be taken is chosen with minute care. It must never be taken from a region of the wound which is bleeding. That region is sought for where there is the 
greatest probability of finding microbes. As the smooth surface of muscle is very quickly disinfected, for preference one examines the greyish structures which are found in the deepest parts of wounds, necrosed points of fascia, the surface of damaged bone or the culs-de-sac of irregular wounds, where secretions can accumulate protected from the antiseptic liquid. It is by means of

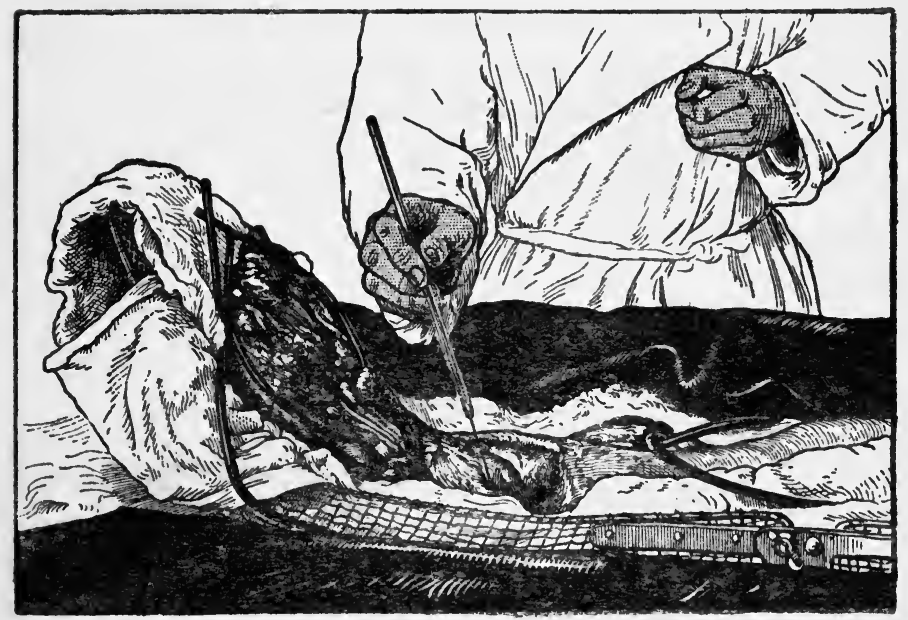

FIG. 66.-Taking a specimen.

multiple specimens taken from various parts that one can ascertain the bacteriological condition of a wound. In surface wounds, it is useful to examine the neighbouring skin. With the aid of a bistoury or a rigid platinum wire the surface of the skin or the epithelial border is lightly scraped.

2nd. Preparation of the Slides. - The secretions thus collected are spread out on microscope slides (Fig. 67), 


\section{I84 TREATMENT OF INFECTED WOUNDS}

which are furnished with a label upon which are written the name of the patient, his number, the character of the wound, and the region of the wound whence the secretion was taken. The slides, thus prepared cluring the course of a round of visits, are arranged in a box for microscope specimens, where they dry, and are taken to the laboratory, where a nurse fixes and stains them.

Each slide is held between the thumb and indexfinger, and passed three times through the flame of a

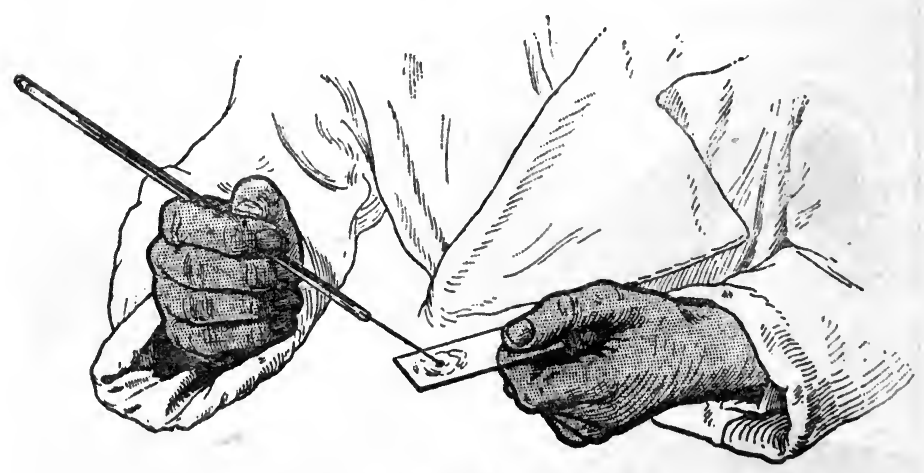

FiG. 67.-Making a "smear."

Bunsen burner, the smear being turned towards the flame.

Then it is placed on a glass support and receives a few drops of carbolised thionin. After half a minute, it is washed with water and put aside to dry.

3rd. Counting the Microbes.-The slides thus stained are arranged upon a table, and the nurse places on each smear a drop of oil of cedar. The preparations are then examined with a No. 12 immersion objective and a No. 3 eyepiece. The number of microbes found in the 
field of the microscope are counted, and the anatomical elements which are found there are also scrutinised. This technique gives naturally only crude results, but they are adequate. In fact, when the number of microbes per microscope field exceeds fifty or a hundred, it is useless to count them more precisely. The examination of the smears has but one object, to indicate the progress of treatment. Hence it is easy to note that a secretion, one day containing innumerable microbes, shows the next day a marked diminution in their number. Should the number drop below fifty per microscope field, counting is easier. When it is a question of closing a wound, half a score fields should be looked over carefully. When the smears no longer yield microbes, or only one to five or six fields, then the surgeon should be notified as to the possibility of suture.

The bacteriological condition of the wound is expressed by a fraction in which the numerator gives the number of microbes observed, while the denominator shows the number of fields examined.

Graphically, the bacteriological state may be represented on a chart, where time is shown in the abscissae, and the number of microbes contained in a microscope field in ordinates (Fig. 68). As it frequently happens that only a single microbe is seen for two, five, or ten fields, this is expressed by $\frac{1}{2}, \frac{1}{5}$, or $1_{1}^{1}$ microbe per field.

Each patient has a chart which informs the surgeon concerning the condition of the wound day by day.

4th. Causes of Error.-Certain mistakes should be avoided when taking specimens. In the first place, haemostasis must be absolute at the moment of taking the specimen. When the secretions are diluted by 


\section{I86 TREATMENT OF INFECTED WOUNDS}

blood, microbes can no longer be discerned. It is for this reason that the "smear" method gives no indication in the great number of cases as to the state of infection of fresh wounds. ${ }^{1}$

So long as haemorrhage persists, it is useless to make smears. Again, in examining wounds of longer standing,

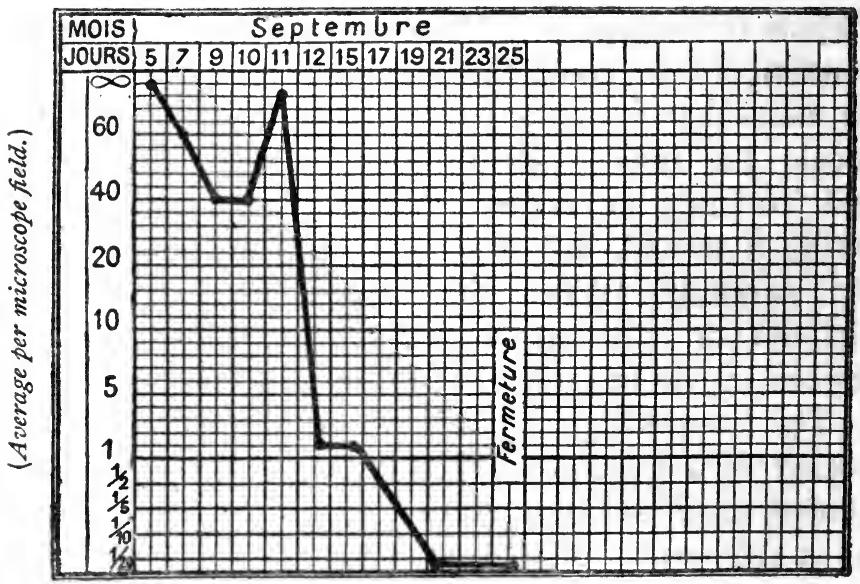

FIG. 68.-Microbial Chart. In the upright columns are noted the number of microbes found per microscope field, varying from infinity to $I$ and from I to $\frac{1}{20}$. A twentieth implies one microbe per twenty fields of the microscope. The upper horizontal column marks the date.

it is necessary to take the specimens at a time when the secretions are not diluted by hypochlorite, and from regions where infection still persists. The smooth surface of muscles is rapidly sterilised, so that specimens taken in those regions do not give a true idea of the real state of the wound. Many specimens from different

1 Ignorance of this detail has caused certain surgeons to believe that fresh wounds are not infected. 
PLATE I.
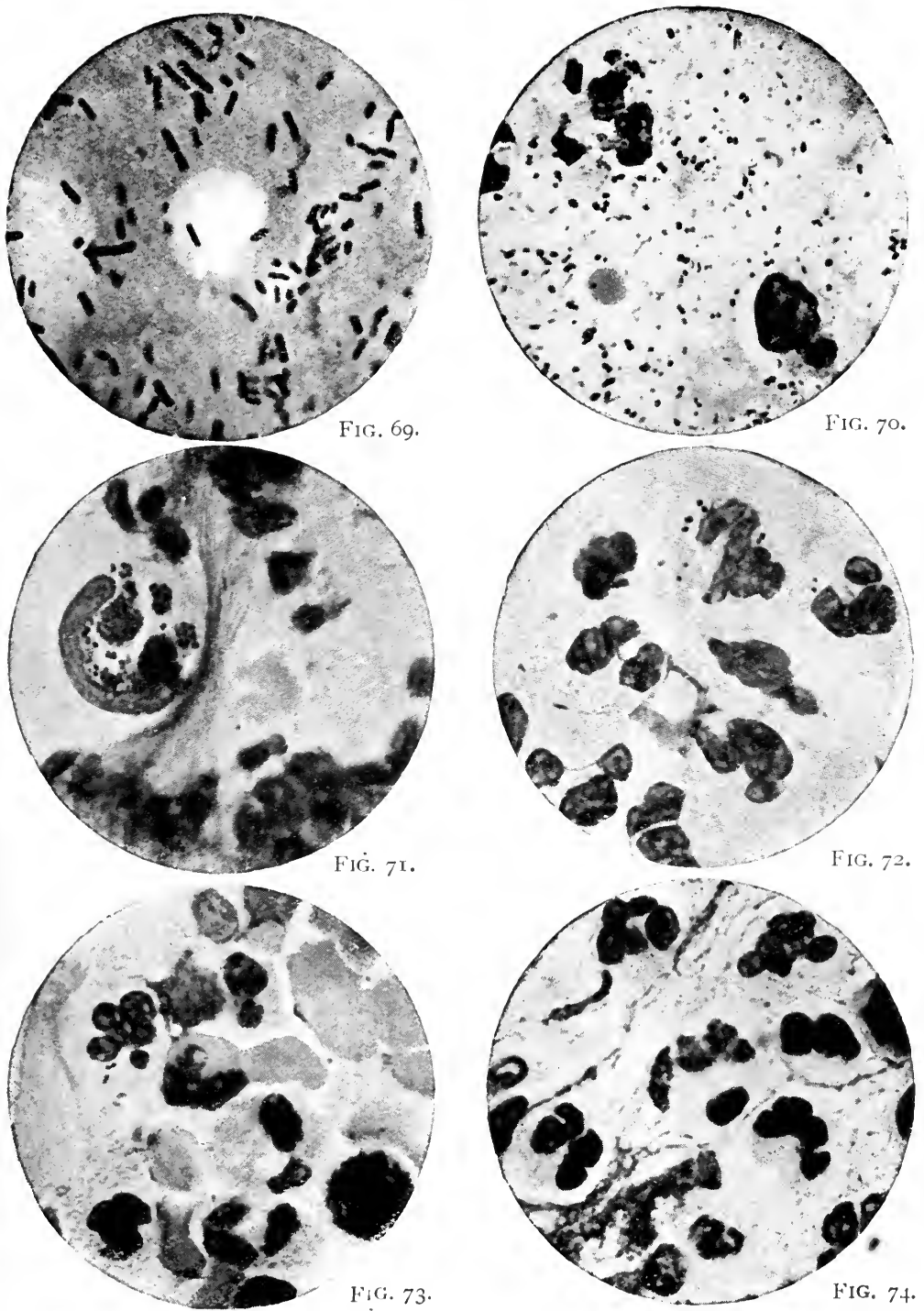

Fracture of the femur in its middle portion by a ball (Case 522). FIG. 69.-May 28. Rod-like bodies in immense numbers. Fig. 70.-May 31. The rods have almost disappeared, and have been replaced by an immense number of cocci. Fig. 7r.June 3. About 30 cocci per microscope field. They are usually in clusters. FIG. 72.June 7. ro to $I_{5}$ cocci per microscope field. Fig. 73. - June I3. About 2 microbes per field. Fig. 74.--One coccus to 10 or 20 fields of the microscope. The wound is aseptic. (The illustrations represent only the central portion of the field of the microscope.) 

regions of the same wound must be taken, to avoid error.

B. Results of the Examination.-The examination of the wound allows us to estimate, according to the bacteriological condition and the anatomical elements present in the secretions, the degree of sterilisation of the wound. By counting every two or three days the microbes contained in secretions taken from different parts of the wound, and by studying the evolution of the leucocytes, the progress of sterilisation can be followed.

Ist. Modifications of the Bacteriological Condition of the Wound. (a) Fresh Wounds.-Smears from wounds less than twelve hours old rarely show microbes. They are only found in the immediate neighbourhood of shreds of clothing on the surface of crushed muscle, and are usually rod-like bodies. They are very few in number, and the microscope field has to be moved several times to discover one. Occasionally when the wounds are six or eight hours old, the smears yield microbes plentifully. If no microbes are seen in the secretions of a fresh wound, it will not do to conclude that the wound is sterile, but simply that the microbes are still few in number and so diluted by blood that they cannot be seen. Experiment has demonstrated that, in cases where the smears are negative, cultures made from fragments of shell or clothing, débris of muscle or connective tissue taken from the immediate vicinity of foreign bodies, give positive results. Almost always, anaërobic cultures made under these conditions produce gas. It might be said that every shred of cloth can determine a gas infection.

At the end of twenty-four or thirty-six hours, the 


\section{I88 TREATMENT OF INFECTED WOUNDS}

secretions of the wound often yield microbes (Figs. 69 and 75). The topographical variations of infection are less marked, and these differences diminish as the secretions become more abundant. Twenty-four or forty-eight hours after the commencement of instillation of hypochlorite, the topography of infection and its volume are

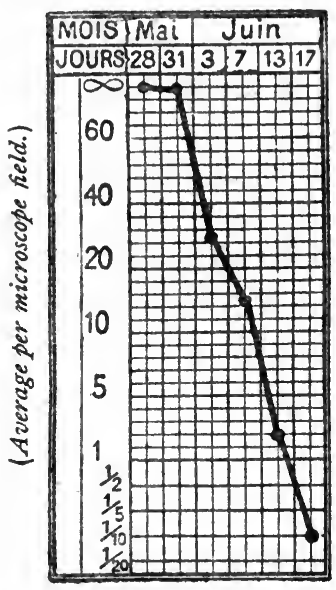

FIG. 75,-Curve representing the sterilisation of the wound in case 522. It shows that the microbes which were in great number on May 3r, diminished and practically disappeared by June 17 .

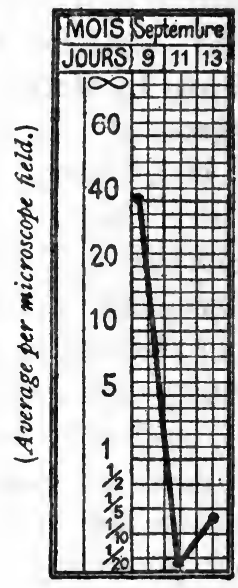

FIG. 76.-Right knee. Wound external. Typical curve of sterilisation of a wound of the soft parts. The wound, which contained 40 microbes per microscope field at the time of the first examination, was surgically sterile two days later.

modified. The surface of normal muscle only shows half a score microbes per field, whilst they are beyond counting on the surface of fractured bone and especially in the debris of necrosed muscular or cellular tissue. After two, four, or six days, the greater part of the surface of the wound is sterile, but microbes remain on irregular bony surfaces, and in deep culs-de-sac which 
have not been reached by the liquid (Figs. 71 and 72). Fragments of necrosed tissue still contain the same quantity of microbes. The moment the solution of these tissues by the hypochlorite is achieved, there is an abrupt drop in the bacteriological curve, and sterilisation will then be complete in one or two days.

In wounds of the soft parts, microbes disappear from

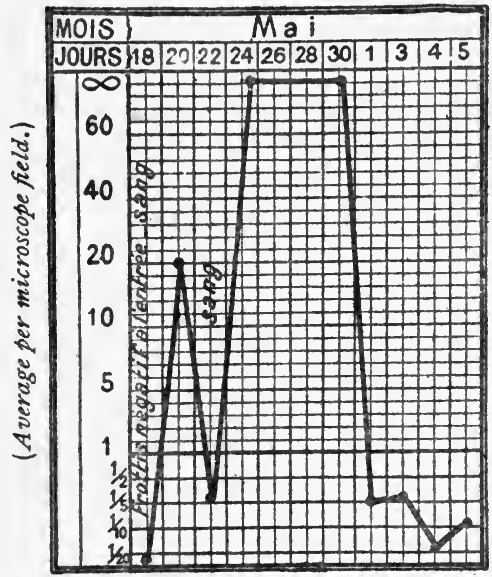

FIG. 77.-Compound fracture of tibia. Sterilisation curve of a highly com. minuted fracture of the tibia. The first smear was negative because the wound was fresh. The third was equally so because of the presence of blood. Sterilisation the ninth day.

the secretion, generally, from the third to the tenth or twelfth day (Fig. 76). In very irregular wounds, and in those associated with injuries to bone, microbes persist much longer (Figs. 69-75 and 77). The microbes disappear at first from the muscles, and from normal cellular tissue, afterwards from the bony surfaces. The last places in which they are to be found are on fragments of necrosed tissue, and more particularly on tendons and 


\section{I9O TREATMENT OF INFECTED WOUNDS}

fasciae. As a general rule, after eight, ten, or twelve days, the entire surface of the wound is aseptic, except where there are still necrosed aponeuroses or narrow and deep tracks. In these cases, instillations of hypochlorite have not the power to alter the topography of infection.

The aseptic cicatrisation of a wound presenting a constant source of reinfection, such as the opening of a sinus unceasingly discharging microbes, can thus be followed. For example, on the surface of a large wound of the abdominal wall there opened a narrow sinus leading down to the fractured ilium.

The surface of the wound was aseptic, whilst the secretions collected at the mouth of the sinus still contained a great number of microbes. The wound therefore showed two quite distinct zones, one aseptic and the other infected. The instillation was continued for several weeks. From time to time reinfection came from the sinus, but it only lasted a few hours, because the septic part of the surface of the granulations became almost immediately sterilised by the solution. The infection was thus kept within narrow bounds at the mouth of the sinus, and the whole of the vast abdominal wound became cicatrised like an aseptic wound.

An analogous limitation of the infection to a very small part of the wound has been observed in many cases. But if, at this period of sterilisation, the instillations are stopped, total reinfection of the wound takes place in a day or two. When the cases whose wounds are almost completely sterilised are transferred to a hospital where a different method is employed, suppuration appears at the end of from two to three days. It is therefore necessary to continue the sterilisation until it 
is complete. If, on the surface of a wound almost completely aseptic, there still persists the mouth of a sinus leading, either to a bony lesion, or to a fragment of necrosed tissue containing microbes, or to some infected foreign body, reinfection always follows without fail directly the instillation is stopped. On the other hand, careful instillation of the solution into a wound allows it to become cicatrised as rapidly as if it were completely aseptic, even in cases where there exists a region still containing microbes.

It is equally important to examine the surface of the skin surrounding the wound. Completely aseptic wounds may become reinfected after the instillation is stopped, because on the surface of the epithelial border and the adjoining skin are many microbes (Fig. 78). These reinfections of cutaneous origin may greatly retard the progress of cicatrisation (Figs. 79 and 80). An examination of the epithelial scales which cover the skin near the wound, shows that they are loaded with masses of microbes. That is the reason why it is essential to wash with neutral oleate of

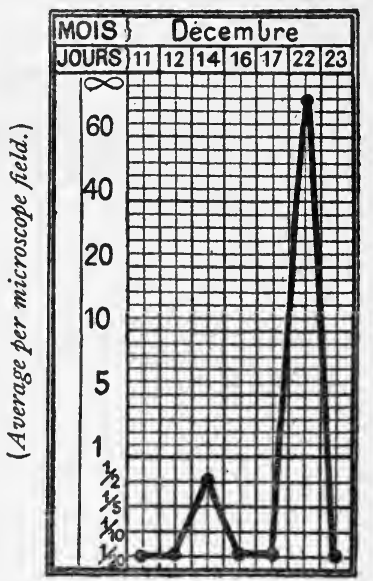

FIG. 78.-Burn. Curve showing a reinfection of cutaneous origin upon a surface wound previously aseptic. soda, not only the surface of the wound, but the whole of the region adjoining.

In short, examination of the smears of the secretions 


\section{TREATMENT OF INFECTED WOUNDS}

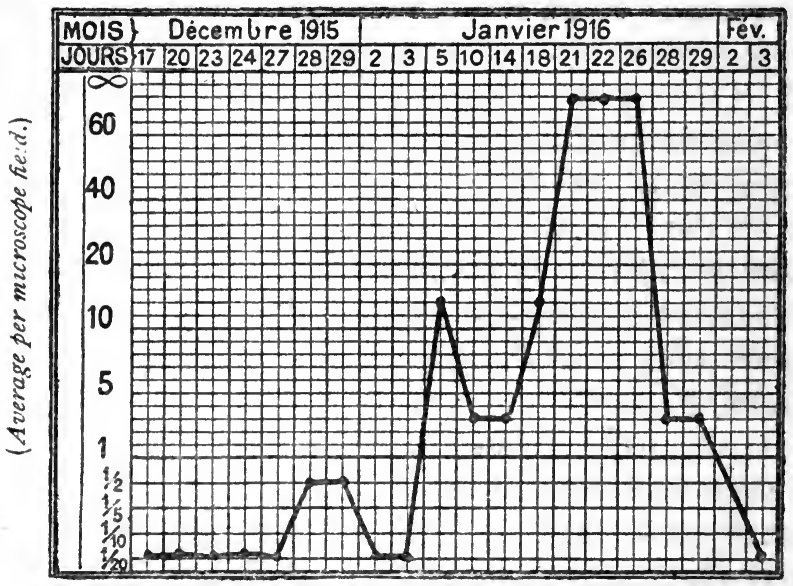

FIG. 79.-Curve showing a prolonged reinfection of cutaneous ollgin.

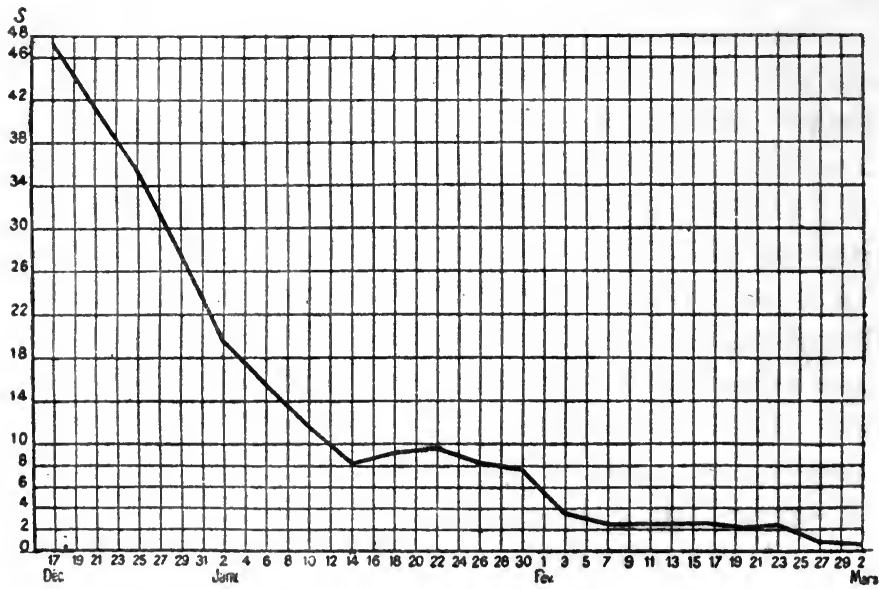

FIG. 80.-Cicatrisation curve of the preceding wound. It is seen that the cicatrisation has slowed down considerably from Jan. I4 to Feb. 28, and that the slowing down coincides with the period of reinfection indicated by the preceding microbial curve (Fig. 79). 
collected from different parts of the wound, and of the results of the scrapings of the skin and epithelial margin, shows, from the time of the instillation of the antiseptic treatment, profound modifications in the topography of the infection. Microbes disappear completely from the greater portion of the wound, but still persist in the necrosed tissue, upon irregular bony surfaces, and upon the skin. As soon as the necrosed tissue is dissolved by the hypochlorite, the microbes which were in this nidus disappear also. Those on the skin and osseous surfaces persist longer. It is necessary, therefore, before looking upon a wound as aseptic, to examine those regions which are the last strongholds of infection, and not to stop the treatment before being quite sure that microbes have been eliminated from the whole extent of the wound.

Variations in quantity alone of microbes are to be considered, because the hypochlorite destroys microbes without distinction of species. Nevertheless, in the course of sterilisation, modifications in the aspect of the microbial flora may be seen. During the first two or three days, the smears contain rod-like bodies, which are often bacilli of Welch, and cocci (Fig. 69). Next, the cocci increase in number, while the rods completely disappear (Fig. 70). Now on the microscope field are to be seen nothing but isolated cocci, diplococci, clusters of staphylococci (Fig. 7I), and chains of streptococci. Under the influence of the antiseptic the number of microbes diminishes (Figs. 72 and 73), and finally a few diplococci alone persist for a few days, then disappear completely.

(b) Suppurating Wounds.-In wounds which have 


\section{TREATMENT OF INFECTED WOUNDS}

reached the suppuration stage before the treatment was begun, the topography of infection is nearly uniform. Specimens taken from different regions indicate everywhere the presence of an almost equal number of microbes. Every morphological variety is represented. The microbes are sometimes isolated, sometimes in clusters, or again within the leucocytes. Sometimes they are so numerous that they form, under the microscope, an almost continuous layer. At the same time, the quantity of microbes contained in pus is extremely variable, according to the treatment which the injury has received. We have examined secretions from the wounds of casualties arriving in the Paris hospitals after having been treated in the field hospitals at the front by the usual means, such as ether or saline solution. All these wounds were suppurating, and the numbers of microbes contained in the secretions were sometimes so great that any attempt at counting was impossible. We have also examined wounds in a fair way to suppurate coming from hospitals (ambulances, Fr.) where sterilisation by means of Dakin's solution had been practised. As the technique had been imperfectly carried out, these wounds contained pus, but in this pus only some fifteen to twenty microbes were found, and sometimes only three or four per microscope field. Therefore there are considerable differences in the degree of infection, and no clinical sign enables one to distinguish a pus containing a large quantity of microbes from another sample of pus containing only a small number.

When a suppurating wound is being sterilised, the bacteriological curve declines almost immediately, and one 


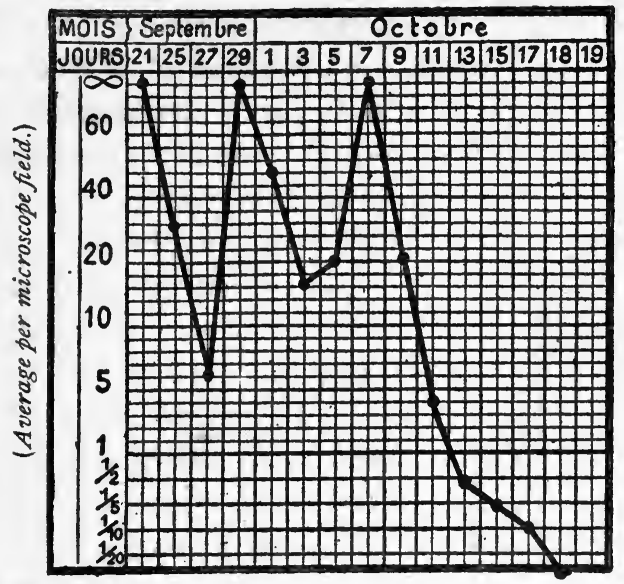

FIG. 8r.-Left calf. Suppurating wound of soft parts, highly infected before arrival at hospital. Slow sterilisation at first, becoming more rapid towards the $15^{\text {th }}$ day.

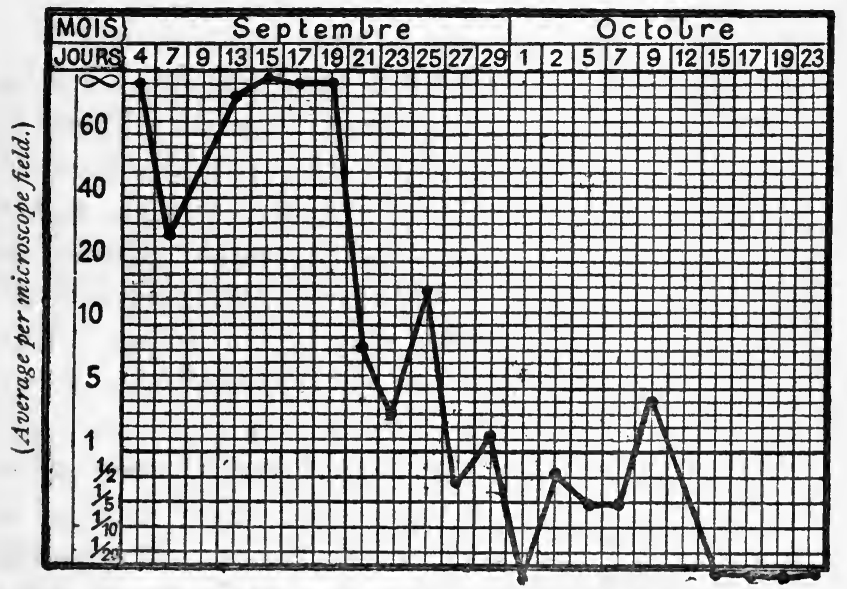

FIG. 82.-Very large, deep, and irregular wound in the posterior part of the thigh and the left obturator region; arrival at hospital in full tide of infection. Almost immediately suppuration disappeared completely. Diminution in the number of microbes became manifest 17 days after the commencement of treatment, and the immense wound was quite sterile and able to be closed 26 days after the entrance of the case into hospital. 


\section{I96 TREATMENT OF INFECTED WOUNDS}

of two phenomena may appear. In the first case the curve goes lower and lower. At the end of a few days the microbes disappear entirely, and sterilisation comes to pass as though a fresh wound were in question (Figs. 8I, 82 , and 83 ). The same evolution may be seen in surface

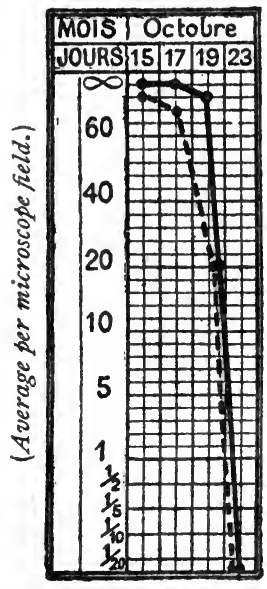

FIG. 83.--ieft leg; wound on internal aspect ....; wound on external aspect - Wounds of the soft parts, suppurating and highly infected. The number of microbes was immense. Sterilisation was obtained suddenly eight days after the beginning of treatment. wounds and in certain deep wounds of the soft parts. But sometimes, after one or several days of almost complete sterility, the pus yields anew a large quantity of microbes (Fig. 8I), which are more often than not in clusters. These sharp ascents of the bacteriological curve are due to the circumstance that little pockets of pus, isolated from the principal cavity of the wound, have become opened and have scattered their contents over the newly sterilised walls. These reinfections are especially observed in very irregular wounds, and in compound fractures. Under the influence of instillation, the microbes may again disappear from the pus, either temporarily or permanently.

In the second case, the bacteriological curve drops under the influence of the antiseptic liquid, then, when it has reached a certain level, becomes horizontal. However generous may be the instillations, the microbes no longer diminish in numbers (Fig. 84). Occasionally in the same patient some wounds become completely 
sterile, whilst others still contain more than fifty microbes per microscope field. The persistence of microbes in the secretions of a wound in spite of the treatment, indicates the presence, in the deeper parts of the wound, of foreign matter, such as shreds of clothing, fragments of projectile, a splinter of bone, a morsel of necrosed tissue ; or perhaps a focus of osteitis

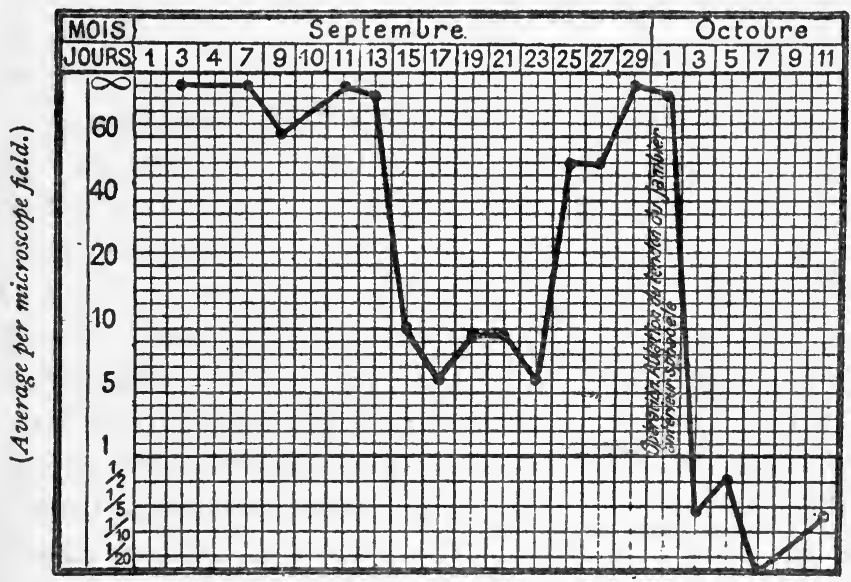

FIG. 84.-Deeply infected compound fracture of the tibia becoming sterilised in a partial manner. The reinfection which occurred from the 23 rd to the 29th decided us to remove a fragment of sphacelated tendon which had been acting as a foreign body. Sterilisation was obtained in two days.

(Fig. 84). If therefore, after having made sure that the instillation of fluid has been sufficient and continued, and that the conducting tubes are correctly placed, it is noticed that the number of microbes does not lessen, an exploration of the wound should be made, in order to remove the foreign bodies, or scrape the bony surfaces which are keeping up the infection; and then the 


\section{I98 TREATMENT OF INFECTED WOUNDS}

instillations of Dakin's solution should be continued. Following upon this interference, the number of microbes increases greatly. Afterwards the curve drops, the microbes diminish in number, and may completely disappear (Fig. 84). In short, every time that a wound does not respond to treatment by a progressive diminution in the number of microbes, it is necessary, after having ascertained that the technique of the treatment has been duly observed, to search for foreign bodies or bony lesions. If the source of the reinfection cannot be traced, complete sterilisation becomes impossible.

To resume, in the greater number of wounds, new or old, the number of microbes diminishes rapidly up to the time when each microscope field contains from one to ten. If at this stage, the instillation is practised in an abundant degree, the secretions will soon show only one microbe to two, five, or even ten fields of the microscope. When the curve does not go down in this way, the cause must be looked for and found.

2nd. Characters of the Leucocytes.-From the twelfth hour, the polynuclear leucocytes are seen in more or less abundance in the wound. During the early days the secretions are composed especially of polynuclear cells more or less altered, of a small number of lymphocytes, and of mononuclear cells. The microbes are free, or within the phagocytes. So long as they persist in great numbers in the secretions, the relative proportion of polynuclear and mononuclear cells changes very slightly.

In wounds treated by hypochlorite or chloramines, the free anatomical elements are altered to the same degree as the microbes. Nevertheless, many polynuclear 
cells enclose microbes which they have devoured before being themselves killed by the hypochlorite. It is therefore probable that the normal phenomena of defence occur as effectually in wounds treated by hypochlorite as in those treated by saline solution. In reality, the hypochlorite does not penetrate into the depths of the tissues. In consequence, in the regions not touched by the antiseptic, phagocytosis goes on as usual. In all the regions affected by the hypochlorite, the leucocytes are destroyed, but inasmuch as the microbes suffer the same fate, no real inconvenience results. Hypochlorite, having a much more energetic action than the fluids of the organism or the polynuclear cells, replaces these agents of defence in their functions. It is ignorance of these elementary principles which has inspired certain French writers with a futile respect for what they call cytophylactic substances.

From the moment when microbes become rare in the secretions, the anatomical elements change their nature. Mononuclear cells increase in number. At the same time we note the appearance of large cells with a single nucleus and fine filaments. It is the indication that the wound is almost aseptic. At the same time, these modifications of the anatomical elements are only of secondary importance in the study of the progress of sterilisation. Although the disappearance of the polynuclear cells and the appearance of large mononuclear cells indicate almost always a marked degree of sterility, the persistence of a large number of polynuclear cells in no way implies that the wound is not becoming sterilised.' In fact we frequently see secretions composed of extremely numerous altered, polynuclear cells which, 
however, contain no microbes. Therefore, to judge the condition of a wound, it is necessary, above all, to base the opinion upon the presence or absence of microorganisms.

C. Value of the Method.-The method we have just described permits a large number of wounds to be examined in a short time. But it is far from being exact. So we must inquire if the simple counting of microbes on a smear gives sufficient information of the bacteriological condition of the wound.

The technique carries with it obvious possible sources of error. In the first place, the smears are of varying thickness, according to the nature of the secretions and the manner in which they are spread out on the surface of the slide. Next, the counting of microbes contained in a microscope field is of necessity far from being exact. If it were a question of finding out precisely the quantity of microbes contained in any given volume of secretion, the method would be absolutely inadequate. But we are not here engaged in scientific research. We desire nothing more than a clinical indication. In a word, the surgeon seeks to learn if the quantity of microbes in a wound under treatment is lessening, and when these disappear completely.

To appreciate the diminution in the number of microbes contained in the secretions of a wound, it is of little moment that mistakes of considerable magnitude may be made in the counting. Errors of ten per cent. or even thirty per cent. are of no great importance. If one day there is found in the pus an uninterrupted mass of microbes, and the next day only a hundred per microscope field can be counted, it is evident that their 
number has lessened. It really matters little that there may have been two hundred or even fifty, instead of a hundred (Figs. 85 and 86). And in the same way, if on the following day one counts ten per microscope field, it is of minor importance that an error of twentyfive per cent. or of fifty per cent. may have been made, because it is certain that the volume of infection has diminished. To sum up, important mistakes in counting do not prevent us from marking on the bacteriological charts the progressive lessening of infection, because the variations in the quantity of microbes under the influence of treatment are very considerable (Figs. 85 and 86-88). Besides, experience has shown that if the examinations are made by the same person under identical conditions, the results are quite consistent, and that the evolution of the wound under treatment can be followed with quite sufficient ac-

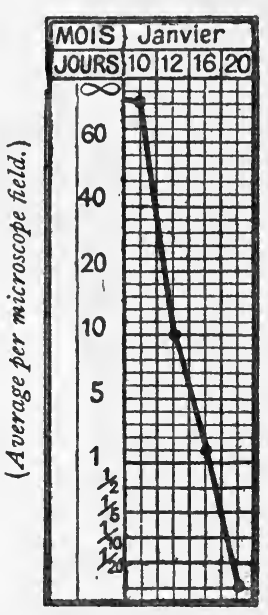

FIG. 85.-Curve representing the sterilisation of the wound in case 318 . Jan. Io, the wound contained a large number of microbes. On Jan. I6 only one per microscope field could be found, and by Jan. 20 microbes had completely disappeared from the smears. curacy.

The date of the disappearance of microbes is indicated with ample precision by the preceding methods. From the time when the secretions contain only half a score microbes per microscope field, counting becomes easier (Figs. 85 and 87 ). It can be done with still more 
precision when only one or two microbes per field are to be found (Fig. 88). If the secretions collected from the different regions of a wound do not contain more than one microbe to five or six fields, the wound may be looked upon as being surgically sterile.

At the same time, clinical signs must not be altogether lost sight of. In reality, a wound whose secretions no longer yield microbes in the smears, may still be infected. When a wound has suppurated during a long period before being submitted to chemical sterilisation, microbes are already encapsuled in the scar-tissue (englobe, Fr.). The surface of the wound may be sterile, while microbes remain latent in the deeper parts. In this case, the clinical history indicates to the surgeon that the deeper portions of a wound, sterile in appearance, may be infected; and that in closing such a wound it is not prudent to make use of deep interstitial sutures, which of necessity would set up reinfection. In wounds which have never suppurated, and of which the secretions are sterile, diverticula may have succeeded in escaping the antiseptic liquid, and may serve as a refuge for microbes. That is the reason why the temperature should always be taken. If a man whose wound is to all appearance sterile has an evening temperature of $37.8^{\circ}$ or $379^{\circ} \mathrm{C}$. ( $100^{\circ}$ or $100.2^{\circ} \mathrm{Fahr}$.), it is probable that a little pocket is cut off from the main cavity and is not completely disinfected.

The disappearance of microbes from the smears by no means implies that the wound is really aseptic. It simply indicates that the degree of sterility compatible with closure of the wound has been attained. We are seeking, in fact, surgical asepsis, not bacteriological 

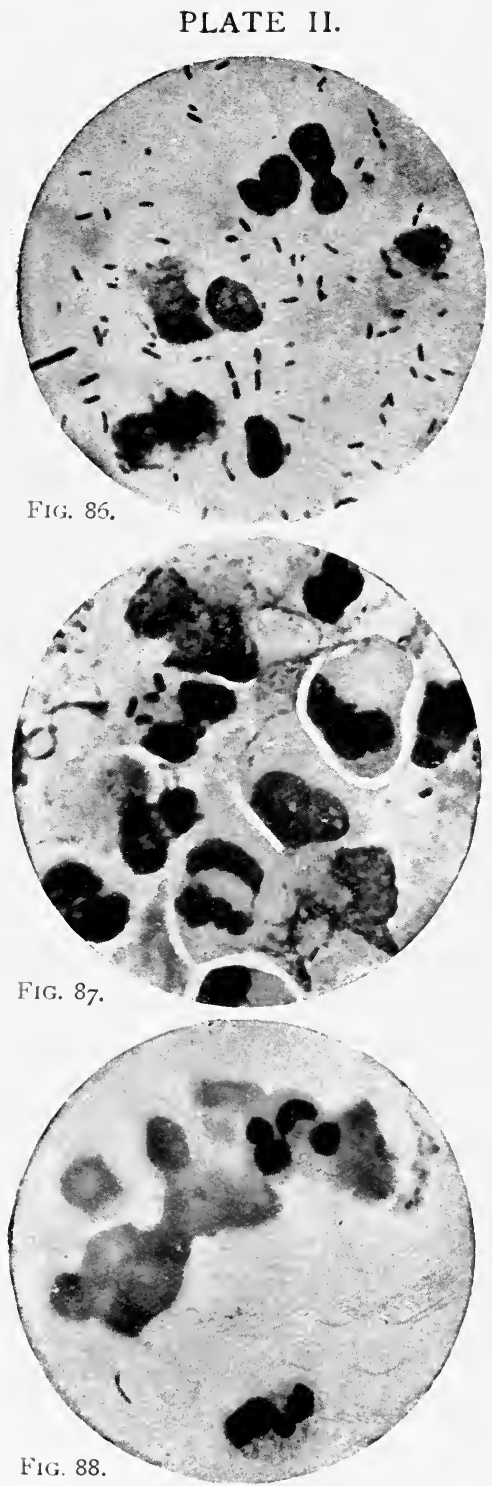

Very large wound of the posterior region of the leg (Case 318). Fig. 86. Jan. Io. More than roo microbes per microscope field. FIG. 87.- Jan. I2. About Io microbes per field. FIG. 88.-Scarcely one per field. (The illustrations represent only the central part of the field of the microscope.) 



\section{BACTERIOLOGICAL EXAMINATION 203}

asepsis. $^{1}$ In the majority of cases, the secretions of wounds whose smears no longer yield a microbe still give positive cultures. Certain writers-for example, Policard ${ }^{2}$-even believe that chemical sterilisation never achieves absolute asepsis of a wound.

Another bacteriologist, M. Tissier, ${ }^{3}$ considered that the absolute asepsis of war-wounds was impossible of achievement, even after protracted treatment. This conclusion merely shows that the technique employed in the cases under M. Tissier's observation was insufficient. By means of suitable technical methods, indeed, the surface of a wound can be rendered so aseptic that cultures from its secretions remain sterile. M. Vincent, 4 in the Compiègne laboratories, carefully examined a certain number of wounds. $\mathrm{He}$ found that in six out of nineteen injuries treated by the usual technical methods by MM. Guillot and Woimant, bacteriological asepsis had been obtained. In short, 30 per cent. of the wounds treated without special attention in the wards of the Compiègne hospital were aseptic. The proportion of bacteriologically aseptic wounds might have been increased by a more meticulous treatment. Whatever the nature of the infection, the wounds became sterile. Those infected by streptococci were sterilised less rapidly than the rest, but they too became aseptic. We do not seek to obtain bacteriological asepsis, ${ }^{5}$ for this is unnecessary, experience having shown that surgical asepsis,

1 Pozzi, Bulletin de l'Académie de Médecine, meeting Jan. II, 1916.

2 Policard, loc. cit.

${ }^{3}$ Debeyre and Tissier, C. R. Socitte de Chirurgie, March 20, 1917.

- Vincent, Fournal of Experimental Medicine, 1917.

- Pozzi, Bulletin de l'Académie de Médecine, Jan. II, 1916. 
revealed by the disappearance of microbes from the smears, is sufficient in practice.

However, by the aid of a precise technique, the surface of a wound can be rendered so aseptic that cultures from its secretions remain sterile. But this degree of asepsis is of no practical interest.

Finally, bacteriological examination in the simplified form we have just described, should be looked upon as an indispensable part of the method of wound sterilisation, because it allows the progress of treatment to be followed step by step, and indicates that it should be modified if the number of microbes does not steadily lessen. Alone, it can point out the moment when a wound may be closed. Indeed, a wound should never be sutured if one is ignorant of what it contains. Despite its lack of scientific precision, the study of smears gives to the surgeon clinical information which is indispensable for the direction of treatment. 


\section{CHAPTER VI}

\section{THE CLOSURE OF WOUNDS}

THE corollary to the sterilisation of a wound is its closure. But a wound should never be closed without knowing what it contains. Suture of a wound enclosing microbes may be followed by downright disaster. It is therefore only after having carefully looked into the bacteriological condition of a wound that one may bring its edges together by strapping or suture.

\section{The TIme For Closure}

Closure of a wound is practised as soon as we know that it no longer contains microbes. Therefore primary closure should be rejected. Even after precise mechanical cleaning of the wound, and resection of every portion which has been affected by the projectile, still it is impossible to make sure that microbes have not been left on the surface of the tissues. So far as that goes, the negative aspect of smears made with the liquids or tissue taken from a fresh wound has no value whatever. A highly infected wound, at this stage, may not show a bacterium upon the slide. Only cultures made by means of tissues carefully collected from numerous points in the wound can give an idea of its bacteriological 


\section{TREATMENT OF INFECTED WOUNDS}

condition. But to have the report of cultures it is necessary to wait twenty-four or forty-eight hours. Consequently it becomes impossible to practise primary union of a wound if we insist first upon knowing its bacteriological condition.

A. Primary Closure of Wounds.-At the beginning of the war primary union was employed, and given up because of the disasters it provoked. Nearly all the cases of septicaemia we have seen were due to immediate suturing performed in clearing-stations or hospitals where its danger was not yet realised. We know that for some months primary suture has again become the fashion in the French Army, and that it has been applied in a great number of cases. It is the fact that the surgeon is often favoured by chance, and that he closes wounds which are only slightly infected, and unite by first intention. But this is not always the case. M. Tuffier reported to the Societe de Chirurgie certain series of cases which revealed 33 per cent. of failures. Over and over again has gaseous septicaemia or streptococcic septicaemia resulted in the death of the patient who has undergone primary suture. The complete statistics have not been published, and only favourable series of cases have been made the subject of communications to the Société de Chirurgie. A batch of cases is regarded as favourable if only I or 2 per cent. of the wounded die, and only one or two amputations are performed. But if we consider that sutures are employed in the case of wounds of the soft parts where the extensive opening and the chemical sterilisation are a sure safeguard against death or amputation, it must be admitted that these results are not satisfactory. As a matter of fact, 
one has no right to make a single patient run an unnecessary risk. And this risk is real, for out of a hundred wounded 'men subjected to primary suture, one or two men have often been killed who would not have died if their wounds had been left open. Only when an experienced surgeon can watch over his patients incessantly, and when there is no danger that the latter may be hastily evacuated, is it permissible to practise the primary suture of the soft parts. This is an exceptional procedure, to be reserved for certain surgical specialists under certain conditions. One of these conditions is a small number of wounded men in the sector in which the surgeon operates. In periods of activity and rapid evacuation this procedure must be completely abandoned. Generally speaking, the primary closing of wounds must be rejected so long as we have no method which will enable us to ascertain whether they are or are not sterile.

B. Secondary Closure of Wounds.-Secondary closure, on the contrary, can be effected under such conditions that it presents no danger. The examination of smears of the secretions of a wound aged twenty-four hours or more enables the volume of infection to be estimated. When the number of microbes has diminished progressively, when it has become zero, and this condition is maintained for two or three days, then we may be sure that an adequate degree of asepsis has been reached, and that the wound may be sutured. At the same time, we must not lose sight of other clinical signs, especially the patient's temperature and the condition of the limb. When the indications furnished by both clinical aspect and smears coincide, then one may suture the wound with a feeling of entire security. 
I. A wound of the soft parts whose sterilisation has been initiated a few hours after infliction, and which has never suppurated, may be closed as soon as two consecutive examinations, made after an interval of one or two days, have shown that the smears do not contain more than one microbe to four or five microscope fields. If the wound be deep, and especially if it be associated with fracture, above all, a compound fracture of the thigh, it is preferable to repeat the examinations and to wait, before closing the wound, until it has been surgically sterile for four or five days.

2. The time for the closure of wounds, the sterilisation of which has been commenced after a more or less protracted period of suppuration, must be more carefully determined. And so far as that goes, experience has taught us that the secretions of a suppurating wound, above all when it is deep and accompanied by fracture, may become for a little while sterile, although the wound is not actually sterile. One day the pus is to all appearances aseptic, and the next day are found indubitable heaps of microbes accumulated on certain points of the smear. In these wounds, which have suppurated for a long time before the commencement of the antiseptic treatment, one should find the secretions sterile for a week at least before deciding to suture.

C. Average Period of Closure.-Generally speaking, the average moment for wound closure occurs between the eighth and the twelfth day. Some wounds may be united towards the fifth or sixth day, others after the twelfth. Certain compound fractures should not be closed before the twentieth or thirtieth day of treatment. It is well to practise the closing of wounds at as 
early a period as possible. As a matter of fact, wounds closed before the eighth day contain no cicatricial tissue, and healing is effected without a legacy of functional troubles. The closure of wounds at an early period also results in considerable saving, both in the cost of treatment and in the work of the staff of the hospital. In a word, as soon as a wound becomes sterile, it should be closed.

\section{Technique of Wound-Closing}

Wounds are closed by strapping, by elastic bands, or sutures.

A: Wound-closing by Means of Strapping.-Co-aptation of the margins of the wound by means of bands of adhesive plaster may be carried out so long as spontaneous cicatrisation has not commenced and the skin moves easily over the deeper parts. It causes no pain to the patient and demands neither local nor general anesthesia. Strapping of American make and good quality is used, four or five centimetres wide, twenty to twenty-five centimetres long. The strips must be long enough to get a firm grip on the skin. As it is not sterile, we must carefully avoid bringing the surface of the strapping into actual contact with the raw surface of a wound, and the line of union is protected by a slip of paper, or of celluloid, sterilised.

The skin adjoining the wound is shaved, thoroughly dried, then the lips of the wound are brought together and maintained exactly in correct position by several bands of strapping applied perpendicularly to the direction of the wound (Fig. 89). At the end of a 


\section{IO TREATMENT OF INFECTED WOUNDS}

week the strapping is removed, and the wound found to be united.

B. Wound-closing by Elastic Traction. - When ex-

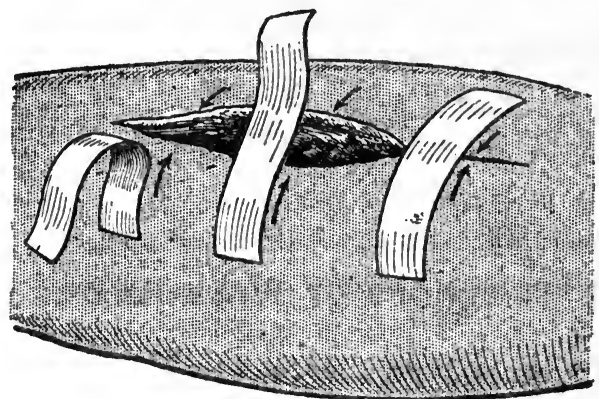

FIG. 89.-Bringing together the lips of a wound by means of strips of adhesive plaster.

tensive loss of substance exists and the lips of the wound cannot be brought into apposition, recourse is had to elastic traction. This method is also used for covering stumps.

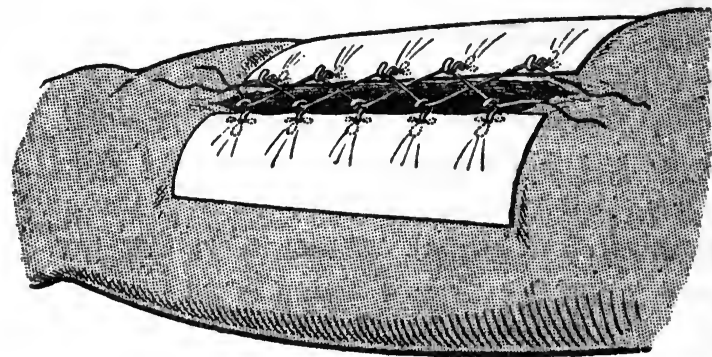

Fig. 90.-Bringing together the lips of a wound by means of elastic traction.

The bringing together of the edges of a wound by elastic traction is carried out in the following manner. Strips of adhesive plaster seven or eight centimetres 
wide (about three inches), and exceeding in length by ten centimetres (four inches) the length of the wound, are provided on one edge with boot-lace hooks, by means of the punch in use by shoemakers. On either side of the wound and parallel to it, a piece of strapping bearing the boot-lace hooks is made to adhere firmly to the skin (Fig. 90). The hooks of the two strips are brought towards each other by means of a lacing of strong rubber, the tension of which is regulated to a suitable degree.

The margins of the wound are brought together progressively under the influence of the elasticity of the rubber. When there has been no loss of cutaneous substance; or when the loss is but slight, the raw surface may be covered in forty-eight hours. When the loss of substance is more considerable, still this procedure allows of the area of the wound being diminished to a very large extent.

A similar method is used to unite the edges of flaps on stumps. It is admitted that, amputations being nearly always practised on an infected limb, the stump cannot be sutured. To check the retraction of the soft parts of stumps left open, we may make use of the method established long ago by American surgeons, that is to say, continuous traction on the skin. Two strips of adhesive plaster of suitable dimensions are applied at opposed points on the surface of the limb, and meet on a small piece of wood $^{1}$ to which traction cords are attached. A weight of about a kilogram and a half (about 3 lbs. English) is sufficient to oppose the retraction of the soft parts. This traction in no 


\section{$2 I 2$ TREATMENT OF INFECTED WOUNDS}

way interferes with the dressing of the wound. When sterilisation is complete it is easy to suture the flaps which are now in the same position as though the amputation had just been performed.

C. Wound-closing by Suture.-Secondary suture of wounds should always be done under anaesthesia. If the skin is adherent to the deeper parts, it must be dissected up to a sufficient extent. To refresh the edges it is enough to remove the epithelial margin by an incision in the healthy skin a millimetre beyond its external border. The simple excision of the epithelial margin will suffice. There is no need to curette the granulating surface. The integuments are dissected up for a distance sufficient to ensure good adjustment of the edges. Usually, the deep parts come together spontaneously. In cases where it may be of service, deep suturing may be practised, especially sutures of aponeuroses. The closure is usually done without drainage, because the bacteriological examination has demonstrated that microbes are no longer existent in the wound.

D. Suture of Muscles and Nerves.-Suturing of muscles and tendons is carried out as early as possible, in order to avoid retraction. It is the same with nerve suture. Directly the wound is sterile, the operative conditions become the same as in aseptic surgery.

E. Closure of Wounds of Compound Fractures or JointInjuries.-In the majority of cases it is possible to close a compound fracture or a wounded joint in the same way as a wound of the soft parts. Even in bad smashes of the limbs, if the closing follows the traumatism by a few days, the osteoperiosteal sac which bounds the 
seat of the fracture has supple walls, which a compressive dressing will so far bring together as to efface the cavity. In these cases suture may be practised without troubling as to the loss of bony substance. However, in certain fresh epiphyseal fractures, and in the majority of old fractures, the osseous cavity has rigid walls, so that it is necessary to fill it up. We have obtained good results with Mösetig's and Beck's pastes (la masse de Mösetig et la pâte de Beck, Fr.). But better results have been observed by MM. Guillot and Woimant as a result of the employment of adipose grafts, the success of which is almost invariable. The inert substances which are used for filling cavities offer this disadvantagethey necessitate a musculo-aponeurotic wall to isolate them from the cutaneous sutures. They have to be worked up and softened by heat; and they yield practically 50 per cent. of failures. Adipose grafts, on the other hand, may be closed in under a simple cutaneous suture. They may, in the event of an extensive abrasion of the soft parts, form part of a cutaneous strip pressed into service from the neighbouring parts. Lastly, the surgeon can always find them ready to hand on the person of the patient himself.

Wounds of joints are closed in the same way. Should one of the bony extremities contain a large cavity, it is filled up in the manner we have just pointed out, before proceeding to the closure of the articulation already indicated. 


\section{The Use of Different Methods according} TO WOUND CONDITIONS

The preceding methods are chosen according to the particular conditions presented by the wounds it is desired to close. These wounds may be divided into different categories according to age and the presence or absence of previous suppuration.

Ist. Closure of Fresh Wounds which have become Sterile before the Twelfth Day.-Wounds of the soft parts may be closed in the majority of cases-that is to say, in nearly ninety per cent. of the cases-before the twelfth day. As, at this period, the skin is movable on the deeper structures, bringing together of the margins of the wound by strapping is habitually practised. Even when the wound is deep and irregular and a fracture is present, the operation is not painful and needs no anaesthetic. Multiple wounds on the same patient may be closed one after another as they become sterile. If the skin has been irritated by tincture of iodine, or the wounds are too close together to allow of the strapping method being applied, we have recourse to suture, and if there is loss of substance, to elastic traction. The method of suture is also employed in fresh wounds, when one has to unite tendons, muscles, or nerves. As anaesthesia is necessary for suture of nerves, tendons, or muscles, the operation is terminated by cutaneous suture. With the exception of these cases, we always use the strapping method, which has the merit of bringing together the deep parts of wounds as well as the superficial portions.

2nd. Closure of Fresh Wounds which have become Sterile after the Twelfth Day.-When the sterilisation 
of the wound has only been achieved after the twelfth day, it is no longer possible to use the strapping method. Suture is then practised. As the wound has been subjected from the outset to antiseptic treatment, and it is probable that the cicatricial tissue contains no microbes, catgut stitches may be put in without danger of reinfection. In wounds which have remained open longer, careful bringing together of the deeper parts is carried out. It is not sufficient merely to approximate the skin. 3 rd. Closure of Wounds which have become Sterile after a Period of Suppuration.-When it has not been possible to apply the treatment from the beginning, and the wound has suppurated for a longer or shorter period, the process of closure must be a little different. In these cases, in fact, numerous microbes have been shut up within the cicatricial tissue. Smears show that the surface of the wound is sterile, but they yield no indication as to the state of the deep parts which are already cicatrised. It is therefore important to bring the tissues together without injuring them, that is to say, without making a deep dissection, and without interstitial sutures. The scalpel or the needle, when traversing a cicatrix which contains microbes, may start reinfection. We must, therefore, be content with bringing together the deep parts by external means, and only suture skin. Also, one may operate in two stages. In the first stage, dissect up the tissues, prepare the wound for closure, loosely insert sutures; then for a few days continue the sterilisation of the wound. In the second, stage, close the wound. By taking these precautions, a result may be obtained as favourable as in the union of wounds which have never suppurated. 


\section{CHAPTER VII}

\section{THE RESULTS}

THE method should only be credited with the results obtained by application in its entirety. If the details of the technique or the composition of the antiseptic be modified at hazard, sterilisation of wounds becomes impossible. The observations made by surgeons who have used Dakin's solution without a precise technique should therefore be looked upon as valueless.

\section{Results of the Sterilisation of Wounds}

Sterilisation of a wound comes to pass in a different manner according as it is recent or old-standing and is associated or not with fracture.

A. Wounds of the Soft Parts.-From the month of December, 1915, the date when the technique was first employed under its actual form as at present, all wounds of the soft parts have attained surgical asepsis. They were subjected to secondary suture, with the exception of those which were very small and healed spontaneously, and those which were accompanied by so great a loss of substance that they could not be closed. Wounds, fresh, phlegmonous, gangrenous, suppurating, all were equally capable of clisinfection, but 


\section{PLATE III.}

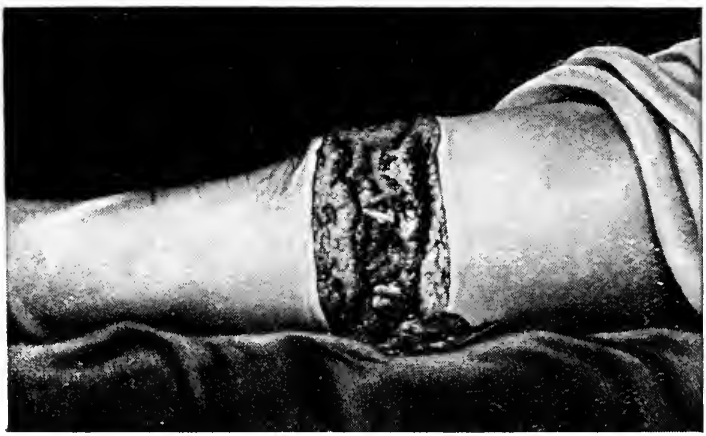

FIG. 91.-Case 465. Section of quadriceps, 3 rd day.

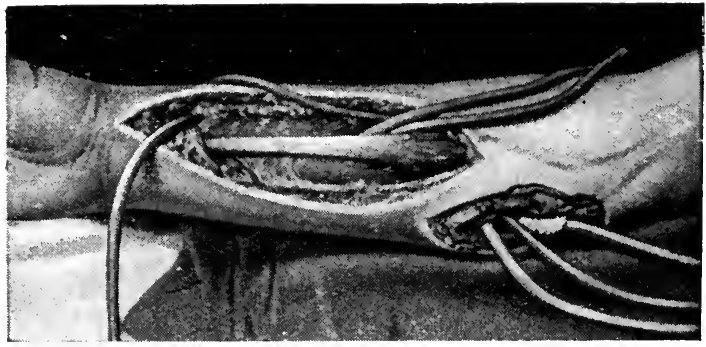

Fis. 93.-Case 6o6. I Iarge wound of forearm.

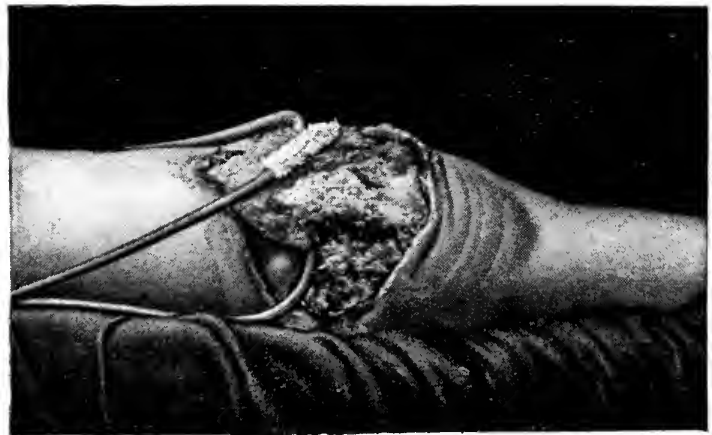

FIg. 95.-Case 577. Wound of knee, $5^{\text {th }}$ day. 


\section{PLATE IV.}

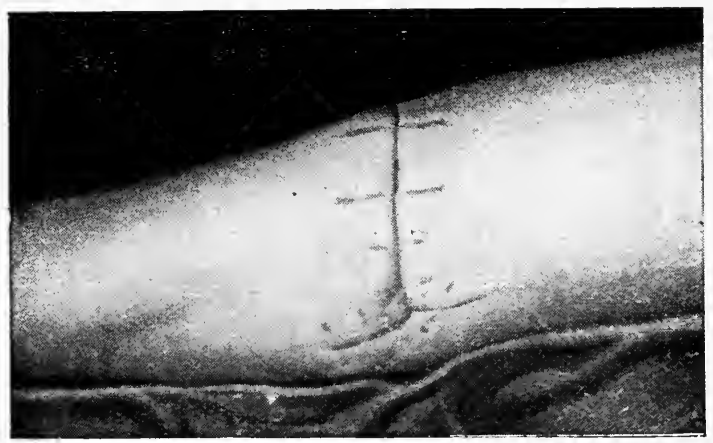

Fig. 92.-Case +65. Suture, $7^{\text {th }}$ day.

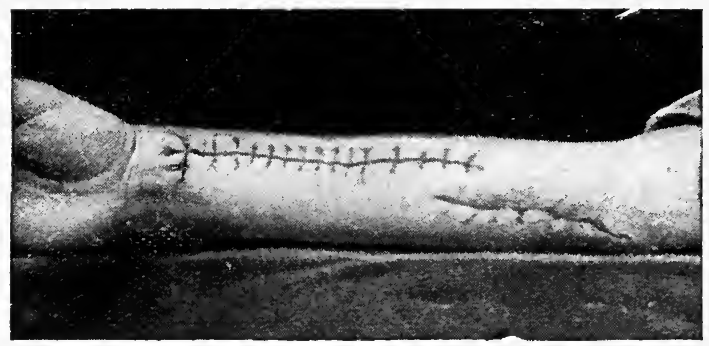

Fig. 94.-Case 6o6. Wound closed, 6th day.

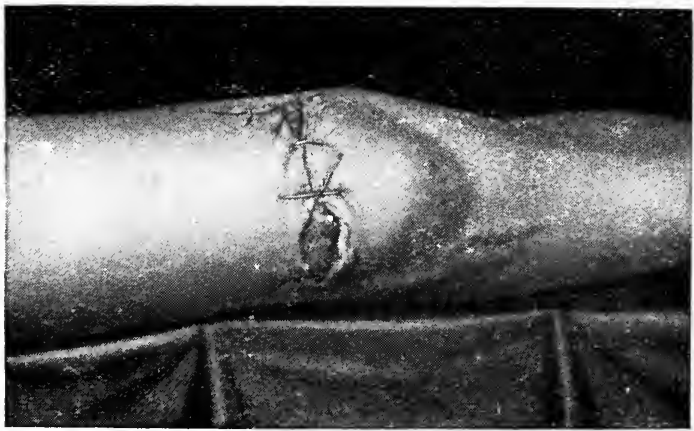

FIG. 96. -Case 577. Suture, I 4 th day. 
the rapidity of the sterilisation depended in a certain measure on the state of the infection.

Ist. Fresh Wounds.-When the treatment of wounds was commenced from five to twenty-four hours after the injury, sterilisation was rapidly produced. Generally microbes disappeared from the fifth to the twelfth day if the wounds contained no gangrenous tissue. The following, which have been chosen from amongst many similar reports, show with what rapidity a large wound can be disinfected and sutured.

Case 465 suffered from a large shell-wound traversing the anterior aspect of the thigh and almost completely dividing the quadriceps femoris. Three and a half hours after the receipt of the injury, the wound was laid open and foreign bodies and torn muscular tissue removed. An extensive wound resulted, more than Io centimetres long and extending from one side of the thigh to the other (Figs. 9I and 92). At the end of seven days, the wound was surgically sterile. Then careful suture by catgut of the quadriceps was carried out and the skin closed (Fig. 92). It healed by first intention, and shortly afterwards the patient walked normally.

Case 3I 5 was operated upon twenty-three hours after having received multiple shell-wounds, of which two were deep in the buttock. The most extensive of the wounds measured after cleaning-up 18 centimetres long, 9 centimetres wide, and 8 centimetres deep. Sterilisation of this wound was slightly retarded by the presence of, gangrenous tissue, which was found near the cutaneous margin of the wound. However, after five days, the wound became surgically sterile, and was closed with 


\section{TREATMENT OF INFECTED WOUNDS}

adhesive plaster. Nine days later, the strapping was removed and the wound found to be healed.

Case 606: a shell-wound penetrated the forearm, went through the epitrochlear muscles and divided the radial (Fig. 93). The wound was closed the sixth day (Fig. 94).

In wounds of the soft parts, sterilisation is almost always rapidly achieved. Out of 136 wounds closed during the period-December, I9I 5, and the commencement of January, I916-I2I were closed before the twelfth day. When the cases were operated upon during the first six or twelve hours, closure was practised still earlier. If the tissues have been severely torn by the projectile, and have become gangrenous over a large area, sterilisation is attained more slowly. In case 577 two shellwounds had lacerated and detached all the tissues of the front of the knee, without fracture of the patella (Fig. 95). The projectiles having been removed from the articulation, the wound could be closed the fourteenth day (Fig. 96). In wounds sterile over almost the whole extent of their surface, microbes often persist near the aponeuroses and necrosed tendons, and prevent closure being carried out. This slow elimination of shreds of necrosed tissue was the commonest cause of delay in sterilisation of wounds of the soft parts. That is the reason why thorough surgical cleansing of the wound is so important. In cases where necrosed tissue had remained for a long period on the surface of the wound, suture was practised, as a rule, from the fifteenth to the twentieth day.

2nd. Phlegmonous and Gangrenous Wounds.-Cases arriving later at the hospital, with wounds already bearing 
evidence of phlegmon or gangrene, were treated in a similar manner. After the disappearance of serious infection, many injuries could be sutured.

Case 340, with multiple shell-wounds, was operated upon after nineteen hours. Wounds of the thighs and legs were freely laid open, shell-fragments removed and instillation tubes placed in the tracks. Three of the wounds developed along normal lines and were closed on the ninth day. The fourth, situate at the inferior extremity of the right thigh, suffered a grave complication. The projectile had opened a vein in the popliteal space, and caused a haemorrhagic infiltration of the whole of the cellular tissue of the calf. This haematoma had remained undetected at the time of operation. But, after twentyfour hours, the temperature reached $40^{\circ} \mathrm{C}$. (103. $5^{\circ} \mathrm{Fahr}$.). The calf and the popliteal space were purple, and very painful. The inflamed region was then incised from the popliteal space to the lower third of the leg (Fig. 97). At the end of eleven days, the great wound had become sterile, and the temperature came down from $40^{\circ}$ to $37^{\circ} \mathrm{C}$. $\left(103.5^{\circ}\right.$ to $98.5^{\circ} \mathrm{Fahr}$.). Next, along the margins of the wound elastic traction was applied, for the tissues were too far retracted to allow of immediate union. Under the influence of elastic traction, the margins of the wound steadily approached each other, and united three days later, that is to say, twentyone days after the infliction of the injury (Fig. 98). Sterilisation came about more slowly than in an ordinary wound. However, it should be looked upon as rapid, taking into consideration the gravity and extent of the infection.

Similar results were observed in cases of gangrenous 


\section{TREATMENT OF INFECTED WOUNDS}

infection. Case 454 presented fourteen wounds of the lower limbs, due to the explosion of a grenade. He was operated on six hours after the injury. All fragments of missile were removed, and each wound was furnished with an instillation tube, with the sole exception of a tiny one which was overlooked. The thirteen wounds treated antiseptically developed in normal fashion and were rapidly closed. But the wound which had not been treated was followed by a serious infective complication. This wound was on the external aspect of the right leg. The fragment of grenade was found at a depth of two centimetres in the long peroneal muscle. The track had been carefully exposed and excised, but no instillation tube had been inserted. Next morning, the dressing had an unpleasant odour, and the calf was red, tense, and swollen. Gas escaped from the orifice. A free incision was made on the external aspect, and it was found that the muscles of the front of the leg, as well as the lateral peronei, had been attacked by gas-producing gangrene throughout almost the whole of their extent. Infection had clearly started from the non-irrigated point. Instillation tubes were placed in the wound, which rapidly cleaned up. At the end of six days, the temperature of the case was normal, and the necrosed tissues in a fair way towards elimination. After a second period of six days, the wound was clean and red. Some microbes only remained near the extensor tendons. Twenty-eight days after the injury, the wound was completely closed. The thirteen other wounds had been able to be sutured the twelfth day. Hence in spite of the serious character of the infection, sterilisation only demanded a little more than double the normal time. 


$$
\text { , }
$$


PLATE V.

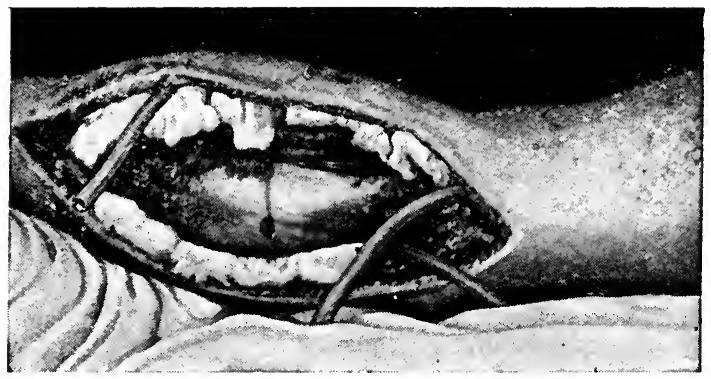

FIG. 97.-Case 3to. Large infected wound of calf, I Ith day.

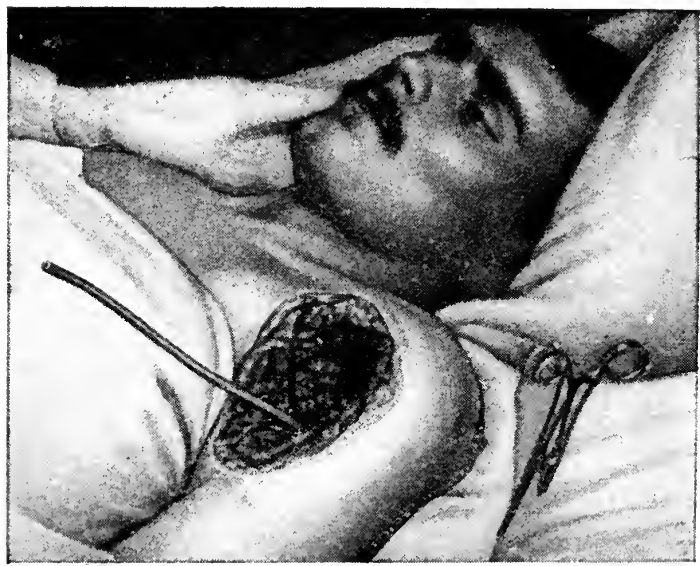

FIG. 99.--Case 433. Fracture of neck of humerus, $5^{\text {th }}$ day.

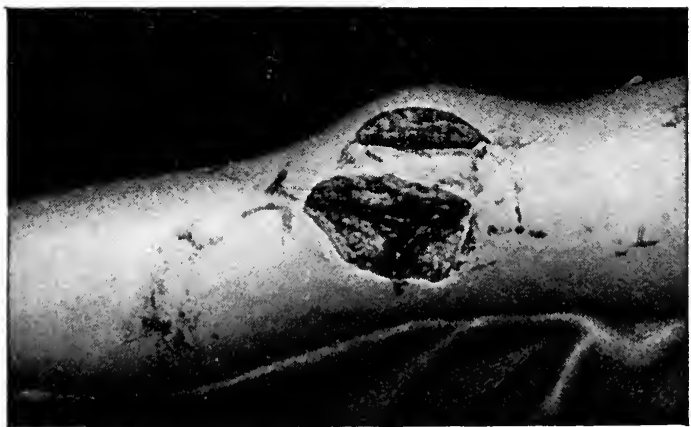

Fif. ror.-Case 594. Shell-wound of knee: partial fracture of condyle, 6th day. 


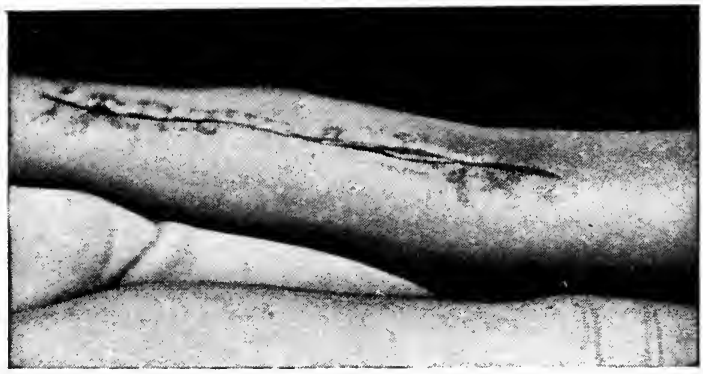

FIG. 98.-Case 3 - 0 . Same wound, the 21 st dily.

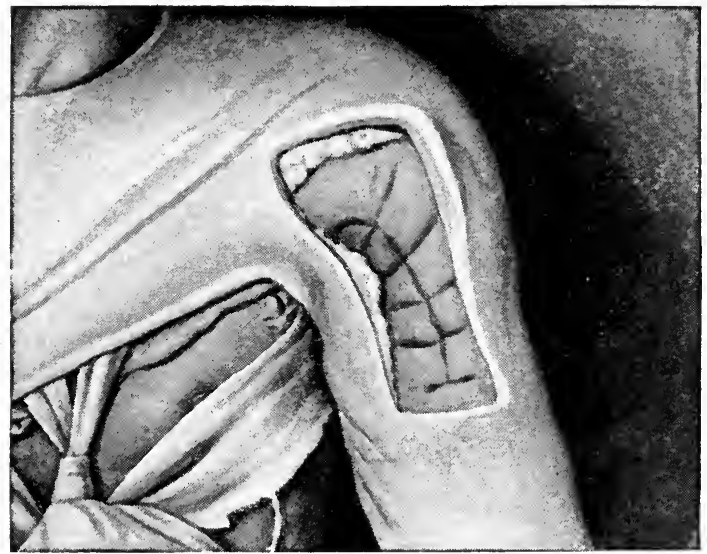

Fig. 100.-Case 433. Suture, i7th day.

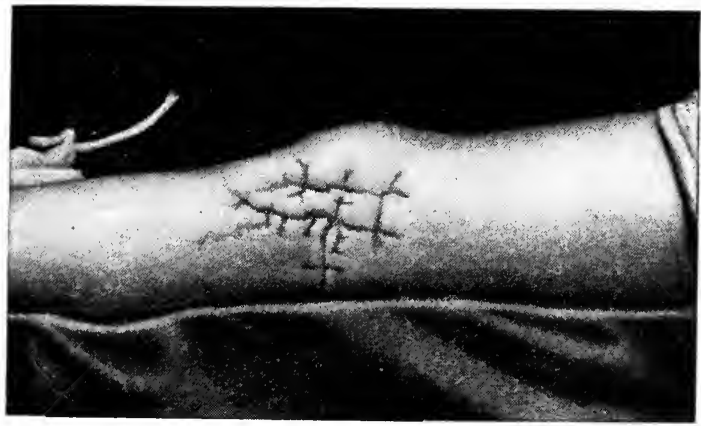

Fig. 102.-Case 594. W'ound became sterile the I6th day and was closed the 20 th. 
3rd. Suppurating Wounds. - Wounds which are already suppurating when brought under treatment are readily disinfected. Surface wounds, even when suppuration is abundant, are sterilised in a few days. Usually, when a granulating wound is washed with neutral oleate of soda, and treated either with hypochlorite or chloramine paste, microbes disappear completely from the smears in two or three days.

It is the same with abscess cavities. When a tube is placed in the cavity of an abscess, and the liquid can reach every portion of the surface of the walls, sterilisation takes place with great rapidity. Then, by a compression dressing, the walls can be brought together and the cavity obliterated in a very short time. When the wound is deep and irregular, and contains necrotic tissue, sterilisation is attained more slowly. In a series of fifty-nine wounds, aged from one to twenty-three days at the commencement of treatment, ninety-two per cent. were closed before the twenty-second day. Some of these wounds were sutured the fifth day, as though they had been fresh wounds. The remaining wounds-that is to say, eight per cent.-were sterilised after the twenty-second day.

We may therefore say that all wounds of soft parts respond to treatment by becoming sterile. About ninety per cent. of both fresh and suppurating wounds were closed before the twentieth day. The rest were disinfected at a slower rate, but all attained surgical asepsis.

B. Compound Fractures.-Results varied according as treatment was commenced before or after the suppuration stage.

Ist. Fresh Fractures.-Experience has taught us that 
from the point of view of results, fractures should be divided into two classes : in one class, short bones, the smaller long bones, flat bones, radius, ulna and fibula; in the other class, fractures of humerus, tibia and femur.

Since the month of December, 1915, we have succeeded in sterilising, in a satisfactory manner from the surgical point of view, all compound fractures of the smaller long bones, short bones and flat bones which arrived at the hospital from five to twenty-four hours after the infliction of the injury, with the exception of fractures of the jaw communicating with the mouth. In the greater number of the cases, fractures of metacarpus and metatarsus, deep wounds of ankle or wrist with laying open of several articulations, have been closed. Fractures of the patella have yielded similar results. We may conclude that these fractures from the sterilisation point of view behave like wounds of the soft parts.

In the majority of cases, sterilisation of fractures of the humerus, tibia and femur has been obtained.

(a) Fractures of the humerus consolidated without its being necessary to make an extensive resection. The possibility of sterilising the seat of fracture allowed the preservation of splinters of orthopaedic value. The greater number of fractures of the humerus, whether implicating or not the articular surfaces, have been able to be sterilised and quickly closed. In highly comminuted fractures, bone fragments which were entirely free were removed, and after sterilisation of the seat of fracture, replaced by Beck's paste. Here is an example of this form of treatment.

Case 32 I came to hospital four hours after having 
received a shell-wound in the right arm. He presented an extremely comminuted fracture of the superior extremity of the humerus directly below the head. The superior orifice was laid open freely and cleansed carefully. A counter-opening on the anterior surface of the arm was made, to remove the projectile, and to take away a large number of small fragments of bone which were lying free. The medullary canal had to be curetted because several splinters had been projected therein. There resulted an extensive loss of substance; three instillation tubes were introduced. After twelve days the patient's temperature was normal, and the surface of the wound no longer yielded microbes to the test. On the fifteenth day the loss of bony substance was made good by Beck's paste, and the wound was closed by a series of intermuscular sutures, and a line of cutaneous stitches (Fig. I03). The twenty-first day stitches were removed. Union was perfect. The man recovered all the movements of the limb.

In non-comminuted fractures of the humerus, suturing was generally done from the tenth to the fifteenth day, and consolidation was brought about as rapidly as in a simple fracture.

(b) In fractures of the tibia, surgical asepsis was attained in a more leisurely fashion. Besides, the loss of integumental substance was often too great to allow of the margins of the wound being brought together. Then we had to be content with sterilising the seat of fracture and awaiting closure by granulation.

In this manner consolidation without a sinus of highly comminuted fractures may be obtained. Case 494 was injured in the middle third of the leg by a shell which 


\section{TREATMENT OF INFECTED WOUNDS}

fractured the tibia. In the course of the first surgical interference by MM. Hornus and Perrin, only the smallest of the free bony splinters were removed, the larger fragments being left lying between the osseous extremities. For nine days, Dakin's solution was instilled every two hours. When the case was brought to the hospital, ten days after receipt of the injury, there

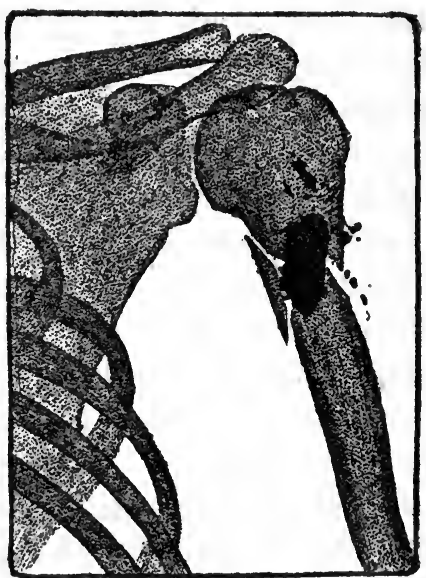

FIG. I03.-Fracture of humerus, filling-in with Beck's paste. Case 321 .

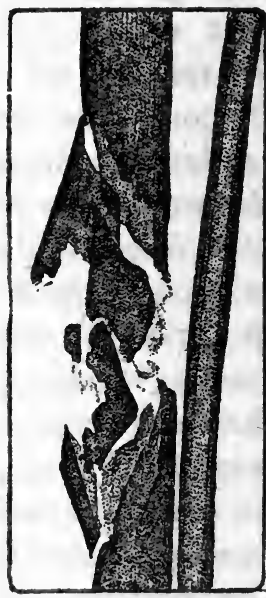

FIG. 104. - Fracture of tibia, Case 494.

were still ten microbes per microscope-field, but the wound bore an excellent appearance, and the tissues presented neither redness nor swelling. The only operative interference was to blunt the point of a splinter which projected into the wound. A month after the injury, all the bony fragments had been covered by granulations, and the instillation tubes were discontinued. By reason of the extensive loss of skin substance 
cicatrisation came about slowly, but two months after the injury, healing was complete without a sinus (Fig. 105).

The conservation of fragments of bone is of great importance from the point of view of ulterior function of the limb. In sterilising splinters more or less denuded,

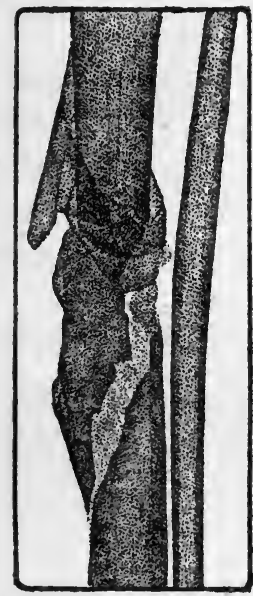

FIG. I05.-Same fracture "healed. Case 494.

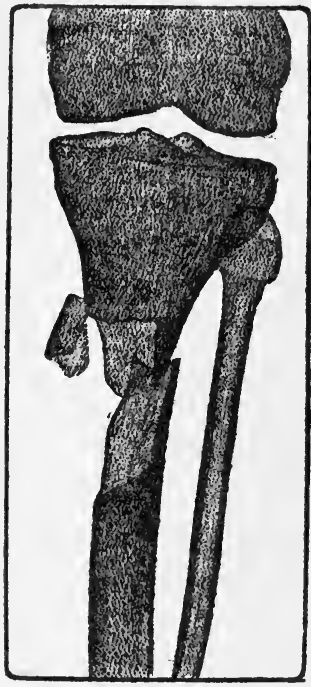

FIG. I06. - Suppurating fracture of the upper part of the tibia. Case 516.

we succeeded in making use of them, and in obtaining consolidation of the bone. In case 516 , a shell had caused a serious fracture of the tibia, at the level of the junction of epiphysis and diaphysis. The internal twothirds of the bone had been destroyed, while the external portion presented two long splinters almost completely denuded of periosteum. They were kept, nevertheless, 
because their ablation would have shortened the bone by seven or eight centimetres (Fig. 106): the anterior tibial nerve and vessels had been severed. The wound rapidly sterilised, but by reason of loss of substance it was impossible to close it. Seventeen days after the injury, there was to be seen at the upper part of the tibia a large wound, at the bottom of which was a bony cavity the size of a small egg. This wound no longer contained microbes. It was then filled with a paste containing chloramine, under which asepsis was maintained. Immediately it was filled up by granulations, whose surface became covered with epidermis. Cicatrisation was complete three months after the infliction of the injury. The fracture was almost completely consolidated, without either reinfection or elimination of sequestra being produced.

In some cases it is possible to close fractures of the leg. In case 627 the fracture of the tibia above the malleoli was found to be sterile ten days after the infliction of the injury (Fig. 107). The eleventh day it was completely closed, and on the sixteenth day the wound had healed by first intention.

(c) Even highly comminuted fractures of the thigh are sterilised in such a manner that in about half the cases suture can be practised. The degree of asepsis obtained in the non-sutured cases was sufficient to allow the seat of fracture to be isolated from the external wounds. Consolidation was produced almost as if a simple fracture had been in question. In none of the cases which reached us during the first twenty-four hours did a sinus persist. Infected fractures of the femur could be closed the I 5 th, $23 \mathrm{rd}$, and the $25^{\text {th }}$ day. 
Case 560 , aged 42 years, arrived at the hospital seven hours after having been struck by a shot which produced an extremely comminuted fracture of the left thigh. The diaphysis of the femur had been broken at the level of its middle third into multiple fragments (Fig. 108). The orifice of entry of the missile, which was

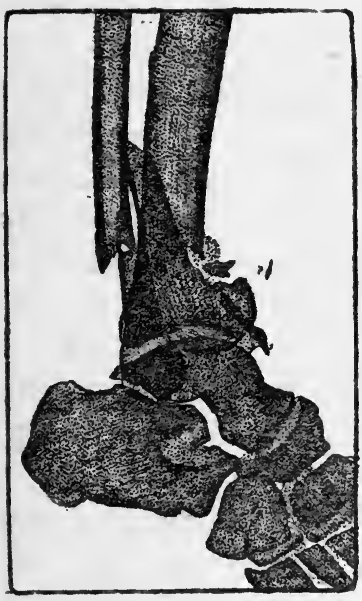

Fig. '107.-Fracture of the tibia. Case 627.

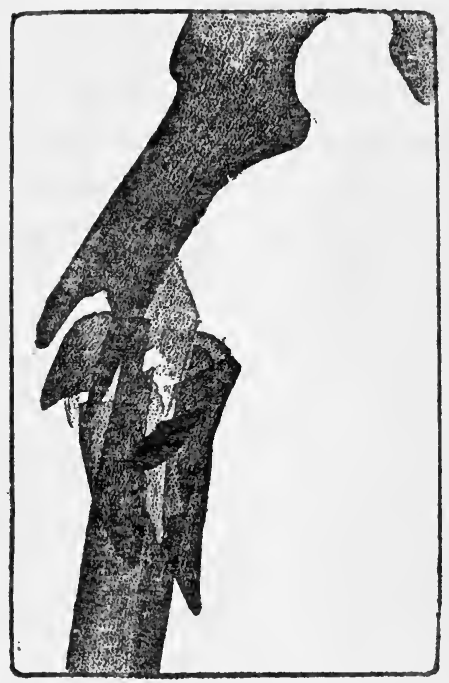

FIG. I08.-Fracture of the femur. Case 560.

internal, was very freely laid open, the contused muscular tissue was excised, and only two small splinters, which happened to be completely free, were removed. Four instillation tubes were placed in the seat of fracture. The temperature never rose above $38^{\circ} \mathrm{C}$. (100 Fahr.) during the first four days, then steadily dropped and became normal. The number of microbes, which the 


\section{TREATMENT OF INFECTED WOUNDS}

second day was 30 per microscope field, diminished to one the $13^{\text {th }}$ day. The $15^{\text {th }}$ day the wound was hermetically sealed by silkworm gut (crins de Florence, Fr.). Union took place by first intention. The fracture was firm on the $47^{\text {th }}$ day.

Another case, No. 495, aged 29 years, had received a wound from the explosion of a mine which fractured the right femur at the junction of the lower with the middle third. Some hours afterwards at the V- fieldhospital, MM. Hornus and Perrin removed some fragments and the foreign body, cleaned up the contused muscles, and placed conducting tubes for antiseptic liquid in the seat of fracture. The 23 rd day, microbes having disappeared from the secretions, the wound was closed with silkworm gut. At this date the case was brought to us (Fig. I09). Union of the edges of the wound took place by first intention. Consolidation was attained, and the patient walked on the 43 rd day. There was three centimetres of shortening, and the knee possessed its normal mobility.

Case 493, aged 38 years, had a fracture of the right femur caused by shrapnel (Fig. I IO). He was first treated at the V- field-hospital, where MM. Hornus and Perrin removed the projectile and some small splinters. Like the preceding, the wound was irrigated by means of Dakin's solution. After seven days he was sent to Compiègne. The temperature was $37^{\circ} \mathrm{C} .\left(98.5^{\circ} \mathrm{Fahr}.\right)$, the wound was a healthy red and presented no sign of suppuration, the surrounding integuments were supple and free from tenderness. At this stage, the number of microbes was about twenty per microscope field. On the 23 rd day it had dropped 
to one per three fields. The wound was closed the $25^{\text {th }}$ day by silkworm gut, and healed by first intention. Consolidation was complete 44 days after the infliction of the injury.

The number of fractures of the thigh treated by us was very limited. But there is no doubt that similar results can be obtained when fresh compound fractures

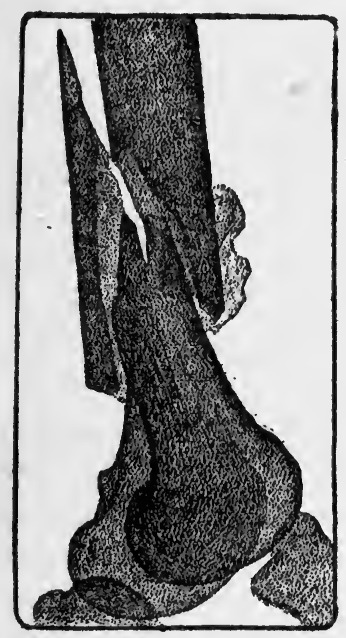

Fig. rog.-Comminuted fracture of femur. Case 495 .

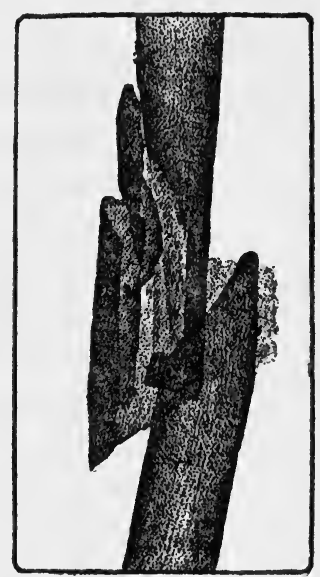

FIG. I Io.-Fracture of femur. Case 493.

are treated by methods similar to ours. Recently M. Hornus treated, in one of the hospitals at the front, thirteen cases of compound fracture of thigh. After four or five days the cases had a normal temperature and no suppuration. In eleven cases, secondary suture was practised: We have seen also, with $\mathrm{M}$. Depage at the hospital of La Panne, fractures of the 


\section{TREATMENT OF INFECTED WOUNDS}

thigh which had attained surgical asepsis and been closed.

It appears, therefore, quite evident, that in fractures of the thigh, and with still more reason in fractures of the tibia and humerus, it is quite possible to avoid suppuration while making very limited resections (esquillectomies, Fr.). Consolidation comes about rapidly, and cases are protected at the same time against pseudarthrosis and the interminable suppuration which so often follow compound fractures treated by the ordinary methods.

2nd. Fractures accompanied by Wounds of Joints.When a wound was associated with the opening of an articulation, results differing according to the region were observed. When the lesion was limited to synovial membranes or to the edges of the articular condyles, arthrotomy followed by disinfection of the seat of fracture permitted the osseous extremities to be retained. Often functional integrity remained complete. The scope of simple arthrotomy could be enlarged, and the number of resections diminished.

In the cases of deeper osseous lesions and more extensive fracture of the articular condyles, we have also been able to avoid resection, and to preserve all the movements of the articulation. Here are two examples of compound fractures of the inferior extremity of the humerus completely restored by sterilisation of the wound.

Case 433 , aged 25 years, had a fracture of the neck of the humerus, due to a fragment of shell which remained in the joint. Four hours after the infliction of the injury, the orifice was laid open freely, the walls of the track 
cleaned, and a resection performed, limited to the detached fragments of bone. After the missile was removed, tubes were placed in the seat of fracture. After fifteen days (Fig. 99) sterilisation was attained, and two days later the joint was closed with silkworm gut (Fig. 100). Union took place by first intention. The twenty-fifth day movements of the joint were begun. The functions of the articulation were reestablished completely.

Case 497 had a shell-wound of the right elbow which fractured the humerus, separating the epicondyle and part of the condyle of the humerus. This case was treated at the $\mathrm{V}$ field-hospital by MM. Hornus and Perrin, who were content with sterilising the large wound by means of tubes going down to the seat of fracture. In fifteen days the wound was sterile. It was sutured the sixteenth day with silkworm gut, and united by first intention (Fig. I I I). The movements of the elbow were re-established almost completely. There remained only a slight limitation of exten-

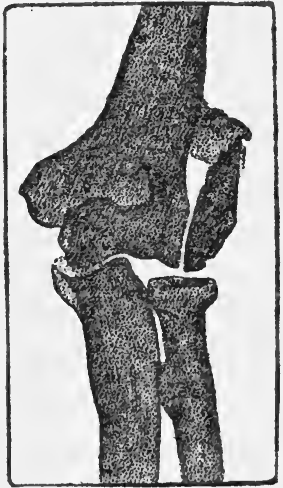

FIG. IIr.-Fracture of condyle of humerus. Case 497. sion.

In wounds of the knee-joint results were observed comparable to a certain extent with those obtained in lesions of the elbow. In those cases where the anatomical conditions permitted, we have endeavoured to sterilise the articulation in such a way as to retain the normal movements. 


\section{TREATMENT OF INFECTED WOUNDS}

Case 472 had multiple wounds of the soft parts, which were cleaned and disinfected, and a wound of the right knee with injury to the external condyle. A piece of a grenade had penetrated the external surface of the knee, traversed the synovial membranes, and lodged in the thickness of the condyle. The aperture of entrance was freely laid open, the walls of the track' resected, the projectile extracted, and the tunnel in the bone carefully curetted. The articular cavity was then dried and shut off by a compress placed beneath and within the damaged condyle, and an instillation tube introduced to the bottom of the bony track. The wound rapidly cicatrised. The eighth day the compress used for "shutting off" was removed, and on the twelfth day the articulation was closed. Union by first intention followed, and passive movements were commenced on the thirteenth day. The movements of the joint were so perfectly restored that the patient walked in a normal manner when he went out of the hospital.

Case 289 was operated upon twenty-four hours after having received a shell-wound which broke the patella into fragments and displaced the condyles of the femur in an anterior direction without their fracture. The contused soft parts were carefully cleansed and all the fragments of the patella removed; the character of the fragments suggested those of an explosion. A compress was placed in front of the inter-articular line, and two instillation tubes were placed in the cul-de-sac of the quadriceps and in the patellar fossa. The temperature, which was $39^{\circ} 9^{\circ} \mathrm{C}$. (IO2 $2^{\circ} \mathrm{Fahr}$.) on the day of arrival, fell by the fourth day to $37.5^{\circ} \mathrm{C}$. $\left(99^{\circ}\right.$ Fahr.). Similarly, the bacilli and cocci which were numerous in the smears were 
reduced by the seventh day to one per five or six fields of the microscope. The wound was then closed by elastic traction. Cicatrisation was complete by the fifteenth day, and passive movements of the joint commenced.

Case 594 had a shell-wound of the knee with partial fracture of the external condyle of the femur. The wound, which was still infected the sixth day (Fig. 10I), became sterile the fourteenth day, and was sutured the twentieth (Fig. I02).

In the following case, despite the very extensive lesions, we were able to save the lower extremity of the femur.

Case 106, aged 22 years, had had a smashing-up of the lower epiphysis of the right femur, fracture of the left patella, and a large wound of the hand. $\mathrm{He}$ reached hospital in a grave condition of shock some ten hours after the infliction of the injury. Immediate transfusion was resorted to, and interference limited to placing instillation tubes in the crevices between the bony fragments which represented the smashed femoral condyles. Into the seat of fracture was instilled paratoluene sulphochloramine, 3 per cent. His temperature never rose above $39^{\circ} \mathrm{C}$. ( $102^{\circ} \mathrm{Fahr}$.), and became practically normal at the end of a month. His general condition remained good, and his hospital stay ended by healing with an ankylosed knee (Fig. II2). In this case no surgical interference was practised at the outset because of the extreme gravity of the case. However, in spite of, the extent of the anatomical lesions of the knee, recovery took place without the patient's condition causing a moment's anxiety. 


\section{TREATMENT OF INFECTED WOUNDS}

3rd. Suppurating Fractures.-The greater number of compound fractures treated by the usual methods suppurate more or less abundantly. We have examined the effects of chemical sterilisation on a score of fractures which had been previously treated in other hospitals, for periods varying from two to forty-six days. Suppuration generally disappeared in from one to four days

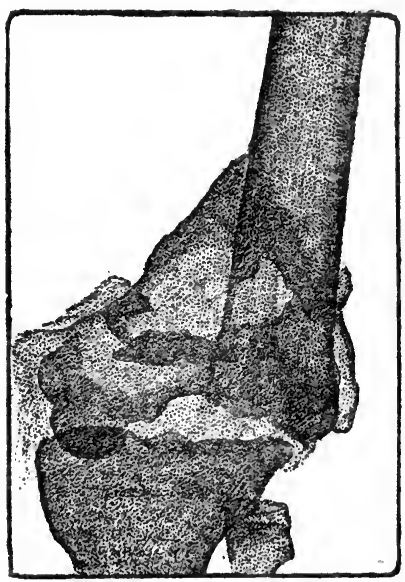

FIG. II2.-Smash of extremity of femur. Case ro6.

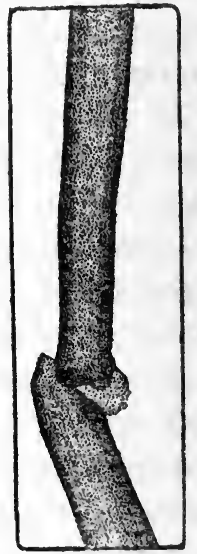

FIG. Ir3.-Suppurating fracture of humerus. Case 624 .

after the commencement of treatment. But the bacteriological curves show that, after the disappearance of the pus, wounds evolve in different ways according to the localisation of the infection.

(a) In the first category of cases, the number of microbes rapidly lessens after the establishment of instillation, and in a few days reaches one per five or six fields. When the curve presents this aspect, there are 
in the depths of the wound neither infected fragments nor a focus of osteo-myelitis, and in spite of the suppuration, the seat of fracture becomes sterile as though a newly inflicted fracture were being dealt with. We have observed this result in several fractures of the humerus, radius, ulna, and some of the smaller bones. Here are two examples of this development.

Case 624, aged 34 years, entered hospital twelve days after a fracture of humerus from shell-wound (Fig. I 13). He had been operated on a few hours after the injury. The left arm had two wounds, one internal, the other external. A big drainage tube traversed the seat of fracture. The limb was surrounded by a dressing stained with blue pus. The wounds were plugged with iodoform gauze, behind which was found a large quantity of pus. The drainage tube was removed, and instillation tubes inserted into both wounds as far as the seat of fracture. The next day the blue pus had, clinically speaking, disappeared. The following day the wounds had taken on the usual red appearance. The microbes, which were innumerable the first day, had completely disappeared ten days later. The two wounds were sutured twelve days after the entry of the patient into hospital. They united by first intention. Two other cases with similar lesions, at the same period, were sutured with like results.

Case 626 presented a semi-section of the upper portion of the forearm, with smashing-up of the two bones. He had undergone operation in a first-line ambulance, and arrived in hospital nine days later. The wounds were suppurating abundantly, the forearm was a little swollen and very painful. The dressings were 


\section{TREATMENT OF INFECTED WOUNDS}

soaked in a large quantity of blue pus. On the surface of the wound remained fragments of gangrenous tissue. The wound was dressed with a paste containing $I^{\prime} 5$ per cent. of chloramine. Two days later the blue pus had disappeared. After three days the swelling of the forearm was gone and the wound was commencing to "clean up." But the microbes were still innumerable. The wound only became surgically aseptic after the lapse of a fortnight. It was sutured the twentieth day, and healed by first intention.

Case 618 , aged 31 years, had a splintered fracture of the femur through the trochanters, due to a projectile which had penetrated the antero-external aspect of the thigh. He was operated on in a first-line ambulance eight hours afterwards. $\mathrm{He}$ arrived at hospital twelve days later. The limb was put up in plaster with a "window." A large rubber drainage tube which was found in the wound was removed, and replaced by three perforated instillation tubes. The patient's condition was good. But the region of the hip was painful and a little swollen. The wound presented scanty secretion which did not yield more than ten to twenty microbes per microscope field. Seven days after the arrival of the case at the hospital the wounds were almost aseptic. Then the two instillation tubes were removed and the wound filled up to the level of the seat of fracture with chloramine paste. The wound became aseptic. We waited until the twentieth day before closing it. Union took place by first intention (Fig. I I4).

(b) In the second category of cases, the number of microbes contained in the secretions diminished rapidly at first, then at the end of a few days the bacteriological 
curve became a horizontal line. The quantity of microbes observed in each field varied from about five to fifty. But they never got below one. When the microbial curve forms a plateau at the level of or above the line indicating five microbes per field, experience has shown that there exists in the depths of the wound either a sequestrum or a patch of osteitis which wbuld justify surgical interference. Even in those cases where

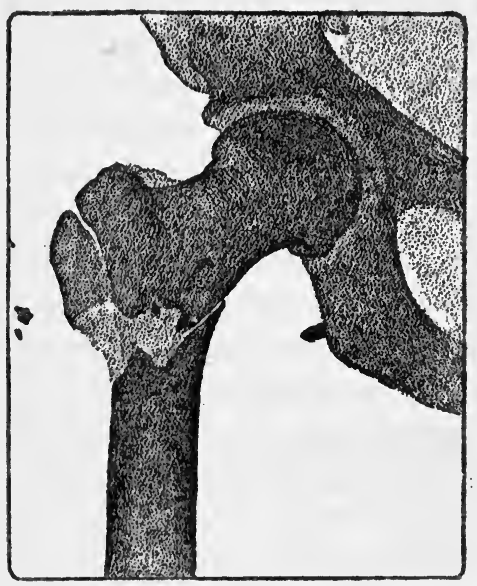

FIG. II4. - Trans-trochanterian fracture of femur. Case 6 I8.

complete sterilisation could only be obtained by resorting to a secondary "cleaning-up," suppuration dried up in a few days and the general condition of the patients changed greatly for the better.

The following example demonstrates how the dura-, tion of treatment may be lengthened if a compound fracture of the thigh be allowed to suppurate even slightly. 
Case 496, aged 25 years, arrived at the hospital forty-two days after having received a shell-wound which had caused a highly comminuted fracture of the right thigh. A few drops of pus came from the opening. Instillation tubes were put in position and the pus disappeared almost completely. But on the surface of the track four or five microbes per field of the microscope persisted. Four months later, slight sero-purulent oozing came from the seat of fracture, from which numerous fragments were removed. Two months later the sinus was still not closed. This persistence of suppuration shows how important it is to sterilise these compound fractures at the outset, to the degree when they contain no microbes at all. In the present case the fracture had consolidated rapidly enough. But care had not been taken to dry up the suppuration in an early stage. The consequence was that the patient, instead of recovering as though he had only a simple fracture, still suffered from a small sinus six months after the infliction of the injury.

When fractures are treated early, even if they are freely suppurating, the results observed are much better.

Case 642, aged 2 I years, had a shell-wound causing fracture of the middle of the right femur. The projectile was extracted in an ambulance at the front five hours after the injury. Two large drainage tubes were placed in the posterior wound and the end of one of these tubes came out by the internal wound. A long anterior wound was plugged with gauze compresses tightly packed in, and almost completely closed by suture over the compresses. The result of this therapeusis was disastrous. When we received the case at the hospital two days 
after the operation the thigh was swollen and very painful. The plaster apparatus and the dressings were soaked in an extremely foetid discharge. The stitches were immediately removed. The tissues were found almost black, covered with sanious pus, stinking. Microbes in infinite number were contained in these secretions. Three irrigating tubes were placed in the posterior wound, three in the anterior, and four in the internal wound. Next day the bad smell had quite gone, suppuration likewise. The following day the general condition of the patient was much improved, although the thigh was still swollen. Six days later, the swelling of the thigh had greatly diminished, and the wound had become red. Eleven days afterwards, some of the tubes were removed, for healing was proceeding rapidly. Twenty-three days after the patient's entrance into hospital, the internal wound was isolated from the seat of fracture, and the posterior and external only communicated with it by a narrow track. Two days later, two of the wounds were sterile, and the third only contained a few microbes. The evolution of this fracture was then comparable, in a certain measure, with that of a fresh compound fracture treated before the onset of suppuration.

In highly comminuted fractures, it was usually impossible to disinfect the wound without surgical interference.

Case 617 , aged 28 , had received a torpedo wound which had pounded up the tibia at its upper part. After some hours it was operated upon in an operating post, where free splinters were removed, and where, very wisely, they had carefully preserved several 


\section{TREATMENT OF INFECTED WOUNDS}

large plates of bone adherent to the periosteum of the internal surface. The seat of fracture was disinfected and dressed with ether, and the limb immobilised in a metallic gutter-splint. This patient arrived at the hospital three days later. The limb looked well and the temperature was $38.5^{\circ} \mathrm{C}$. (101 ${ }^{\circ}$ Fahr.). But the surface of the bone was dark in colour and extremely infected. Examination of the pus showed that the microbes there were innumerable. Two instillation tubes were placed in the cavity, and after four days the temperature fell. Pain and swelling of the limb also disappeared. Nevertheless, after twenty-five days the number of microbes gathered from the surface of the wound was still high. Surgical cleansing of the surface of the bony cavity was carried out, and several small sequestra removed, preserving the periosteum. Instillation tubes were placed in the cavity. Sharply the microbial curve dropped, and reached the level which indicates surgical asepsis.

Even in those cases where the extent of the lesions and the gravity of the general condition do not permit of an integral application of the method, still we can obtain sufficient disinfection to transform both the local and general conditions of the patient.

Case 635, aged 34 years, had a large wound of the right thigh with fracture of the femur. He was operated upon in a field-hospital, where a plaster apparatus had been applied. But a very abundant suppuration set in, and during the weeks which followed, he had seven secondary haemorrhages. This patient reached us forty-six days after the injury. He was in a very serious condition. The thigh presented an antero- 
internal wound and a posterior wound. The denuded extremity of the superior fragment stuck out into the wound. Pus in large quantity poured from the seat of fracture, and rapidly soiled the dressings. The patient was very depressed; his evening temperature was $38.5^{\circ} \mathrm{C}$. ( IOI $^{\circ} \mathrm{Fahr}$.). The urine contained albumen. Haemoglobin was reduced to 30 per cent. of its normal quantity. Systolic arterial pressure was 12.5 and diastolic pressure 8 . In addition the patient suffered from intractable diarrhoea. Because of the gravity of the general condition, we limited our action to slipping four instillation tubes along the bony fragments in the seat of fracture. But the whole of the infected region could not be reached in this manner. As the patient was not in a condition to stand an incision, we were content to irrigate those parts of the infected area we could reach. At the end of a week the general condition had improved, and suppuration had almost completely disappeared. But the diarrhoea changed into dysentery, and the general condition changed for the worse. By way of compensation the local condition rapidly improved. Granulations covered the bare bony surfaces. Pain had disappeared. But microbes remained in considerable numbers. Twenty days after the arrival of the case about 500 grammes of blood were transfused. His general condition improved, and the dysentery, which had been treated by Dopter's serum, disappeared little by little. Twenty-five days after arrival, his temperature was normal and the wounds rapidly healing. Suppuration had not reappeared. This case is a striking example of the possibility of suppressing suppuration, and of thus ameliorating, to a very 


\section{TREATMENT OF INFECTED WOUNDS}

real extent, the condition of a patient who, treated by the usual methods, would have suffered amputation and probably have died.

The treatment of suppurating wounds, accompanied or not by fracture, taken from the convoys going to Paris, has shown us that suppuration can be easily dried up in a few days. Between the 5 th September, 1915, and the $5^{\text {th }}$ January, 1917, we were able, at the Compiègne hospital, to take in 50 fractures, one from three days to eight months after the date of the wound. These were all serious fractures of the thigh, the tibia, or the humerus, some involving the articular extremities. The patients were treated by MM. Guillot and Woimant, who in the great majority of cases succeeded in sterilising the seat of fracture. Fifty-two per cent. of these fractures were sterilised and closed; 42 per cent. spontaneously cicatrised after sterilisation. In 6 per cent, only of these cases there was a secondary formation of fistula. As in these 50 fractures the most serious clinical varieties were included, as streptococcic infections were discovered, and as sterilisation was notwithstanding obtained in 94 per cent. of the cases treated, it must be realised that the method is as fully applicable to old as to recent cases.

\section{Consequences of the Sterilisation of WOUNDS}

The suppression of suppuration and infection in the majority of wounds has important consequences for the patient, since it diminishes to a very large extent the local and general complications of wounds, and consequently 
the length of treatment and the degree of final incapacity.

A. Diminution of the Frequency and Intensity of General Complications.-The rapid sterilisation of wounds nearly always protects patients from those complications which lead to death. From the month of December, I9I 5 , to October I, I9I6, 303 cases of wounded coming directly from advanced dressing posts were treated at the hospital for research at Compiègne. Thirteen died after a stay in the hospital of more than twenty-four hours. In eight cases death was due to extensive anatomical damage to brain, contents of mediastinum, or abdominal organs. In three cases it followed multiple wounds of the two lower limbs, thorax and upper limb. Twice only was it due to septicaemia. One officer, who had a fracture of the thigh with great smashing-up of the bone developed rapid gas-producing septicaemia which in spite of amputation resulted in death. In the second case, staphylococcal septicaemia developed in the train of a fracture implicating almost the whole length of the femoral diaphysis. This case also terminated by death. In all the other cases it was possible to avert serious general infection. It is probable that the improvements which experience has enabled us to make in our methods would allow us to-day to obtain recovery from lesions similar to those which determined the two fatal septicaemias. Lowering of the rate of mortality from infection has been observed by other surgeons who have applied the method in its entirety.

The general condition of the cases whose wounds are in a fair way to become sterilised is habitually good, even when the temperature is more or less elevated. 


\section{TREATMENT OF INFECTED WOUNDS}

This phenomenon was exhibited in a striking manner by those cases which were brought to hospital with injuries of long standing and freely suppurating. Immediately the suppuration disappeared clinically, the general aspect of the patient changed. The first effect of the cleansing of wounds was always marked improvement in the general condition.

B. Dimination in the Number of Amputations.-The suppression of infection has permitted us to escape the lymphangitis, abscesses, and purulent tracks which usually accompany infected fractures and joint-injuries. In a year we have only seen three abscesses. One was the result of a lymphangitis which existed before treatment. The two others developed in the neighbourhood of a fracture of the humerus and of an infected knee. These abscesses were opened, sterilised, and closed in three or four days. In cases where the extent and complexity of the lesions do not permit rapid sterilisation, the destruction of the greater quantity of the microbes and gangrenous tissue immediately produced considerable local amelioration. From this resulted the possibility of preserving limbs which presented very extensive lesions, or of performing conservative operations instead of carrying out radical treatment. In nearly every case where resection of the elbow or shoulder was indicated, we were content with an arthrotomy and disinfection of the articulation. It was the same, to a certain extent, with the knee. In the case of fractures, operations for the removal of splinters have been reduced to a minimum, and thus have been avoided those cases of marked shortening and the pseudarthroses which are so often seen after large removal of bony fragments. 
Amputations have been able to be reduced to the cases in which the crushing of almost the whole of the portion of the skeleton concerned, or the destruction of the vasculo-nervous supply, rendered impossible the conservation of the limb. Between December I, I9I5, and October I, I9I6, we performed twenty-three amputations. These amputations were necessitated in four cases by crushing-up of the bones, accompanied by section of vascular trunks. In sixteen cases it was a matter of limbs being partially or completely torn away by shells, or more especially bombs. The operation consisted in either completing the amputation with scissors, or in amputating a little higher up where the bone became normal. As a general rule, amputation was practised directly through the contused seat of fracture, which the application of numerous instillation tubes allowed to be sterilised in a few days. In only three cases was amputation determined by infection. Two were the cases of septicaemia of which we have already spoken. The third case was a fracture of the upper part of the forearm with extensive vascular lesions and a considerable diminution of the circulation of the limb. This case had been operated on previously in a field-hospital. After a few days the skin became mottled with bluish patches, at the same time signs of septicaemia appeared. Amputation was done, and the patient recovered. Similar results were observed in the field-hospital at V- by M. Perret, who, out of one hundred cases, only amputated once. The sterilisation of wounds, therefore, permits of the preservation of nearly all limbs which are not rendered useless by the extent of destruction of osseous, vascular, or nervous elements. 
Similar, and even more striking results were observed in the case of wounds which were, roughly speaking, from two to forty days old. In the series of a hundred already infected subjects of which we have already made mention, MM. Guillot and Woimant were not obliged to perform a single amputation.

The possibility of disinfecting injuries lessens the number of amputations in a very great proportion, since this operation to-day in 70 per cent. of the cases is caused by septic sequelae.

C. Diminution of Length and Cost of Treatment.-The length of time treatment has to be carried on has been lessened because wounds have been rapidly closed, and because repair of bone, muscle, and nerve has been effected at an early stage.

Ist. Influence of Secondary Closure on the Duration of Treatment.-Wounds of the soft parts, both fresh and suppurating, were closed in the proportion of 90 per cent. from the fifth to the twentieth day, in whatever stage of the wound the treatment may have been commenced. Wounds not sutured in this period were also sterilised, though in a slower manner. If the wounds thus closed during the first twenty days of treatment had been treated by the usual methods, they would have needed from one to six months to cicatrise. By early suture a reduction by about two-thirds of the duration of treatment was obtained.

In compound fractures of flat bones, short bones, and long bones such as the fibula, radius, and ulna, sterilisation was produced as quickly as in the wounds of soft parts. The saving in the length of time needed for treatment was therefore very considerable, because these 
compound fractures, treated in the ordinary way, often suppurated for several months. It is well known how slowly deep wounds of the tarsus, for example, recover when they are infected. One cannot estimate exactly the diminution produced by sterilisation in injuries of the humerus, tibia, and femur. But this diminution is considerable. In fact, compound fractures of the humerus, when sterilised, are often closed after the lapse of from twelve to twenty days, when similar cases, treated by the ordinary methods, are still suppurating after six, seven, or eight months. It is also evident that the closure of compound fractures of the femur after fifteen, twenty, or twenty-five days constitutes a great advance.

A considerable reduction in the duration of the treatment has likewise been effected in other branches of surgery. The possibility of sterilising and closing purulent pleurisies, ${ }^{1}$ as MM. Tuffier and Depage have done, very greatly diminishes the length of the patient's stay in hospital. For the treatment of mastoiditis M. Mahu ${ }^{2}$ has, by means of chemical sterilisation, reduced the term of treatment to one-third of what it used to be. At Pittsburg, Mr. Sherman ${ }^{8}$ has almost entirely suppressed the infection of wounds due to industrial accidents. These wounds are sterilised more readily than wounds received in war, and the saving which may thus be effected is considerable.

2nd. Influence of Early Anatomical Repairs on the Duration of Treatment.-Sterilisation of the wound allows

1 Tuffier and Depage, $C . R$. Socilté de Chirurgie, March 27, 1917.

2 Mahu, Presse medicale, 1917.

3 Sherman, Surgery, Gynecology and Obstetrics, January, 1917. 


\section{TREATMENT OF INFECTED WOUNDS}

us to practise operations quite early in the case which formerly had to be put off until after cicatrisation was complete. Thus bone-grafting or wiring, reunion of muscles or tendons, or nerve suture, before to-day, could only be practised after the healing of the infected wound. This cicatrisation often was only obtained after the lapse of several months. To-day, we set about these reconstructions as soon as the wound is sterile, that is to say, from the eighth to the fifteenth day.

Case 433 presented a section of all the tendons and the median nerve just above the right wrist. He was brought to the hospital three and a half hours after the injury. The wound was immediately cleansed and provided with instillation tubes. After ten days it was sterile. On the eleventh day, all the tendons and the median nerve were sutured, and seven days later, the skin wound was closed without drainage. Healing took place by first intention. This case had at the same time an inter-articular fracture of the right elbow, and a fracture of the left humerus, which were sutured at the same time, and likewise united by first intention.

Reparation of bone tissue may be made with equal safety at an early date. Case 5 I 8, aged 23, had a fracture of the vault of the cranium with a large wound of the hairy scalp. Phenomena of compression disappeared as the result of a craniectomy, in the course of which a fragment of bone the size of a crown-piece was removed. Four days later, the wound having become sterile, M. Woimant made good the loss of bone substance by an osteoperiosteal flap taken from the internal surface of the left tibia. The scalp was closed hermetically, and union took place by first intention. The case was examined 
anew forty days later. It was found that the graft had exactly adapted itself to the cranial wall.

3rd. Diminution of the Cost of Treatment. - The expenses of treatment are considerably lessened, since its length is so much less than by other methods. The saving thus realised is from about 50 to 70 per cent. Besides, the substances used in the treatment are not costly. The net cost of Dakin's solution is three centimes the litre, ${ }^{1}$ whilst ether, alcohol, peroxide of hydrogen and balsam of Peru are very much dearer. Suppuration being done away with, the dressings are but slightly soiled, and almost the whole of the gauze may be saved again for subsequent use. The cost of the appliances for instillation is recovered in a few days from the saving due to the exclusive employment of a substance of such trifling cost as hypochlorite of soda.

D. Diminution of Positive Incapacity.-In the greater number of injuries, eventual incapacity is the result of infection. As the sterilisation of wounds permits the avoidance in many cases of amputations and resections, there results a considerable diminution in the amount of pensions payable to the wounded men by the State. It is also well known that the presence of infection in a compound fracture of a leg or thigh, raises the positive incapacity rate from 5 or 10 per cent. to 25 or 50 per

1 Net cost of ten litres of Dakin's solution:-

$200 \mathrm{gr}$. chloride of lime at I fr. 10 centimes . . . 0.22 $100 \mathrm{gr}$. carbonate of soda (Solway) at o fr. 40 centimes . 0.04

$800 \mathrm{gr}$. bicarbonate of soda at o fr. 60 centimes . . 0.048

Net cost of ro litres . . . . . . . . . . . . . 308

Therefore the net cost of a litre is 3 centimes (rather less than a halfpenny per quart). 


\section{TREATMENT OF INFECTED WOUNDS}

cent., and more. The gain from the suppression of infection is therefore very evident. In successfully treating fractures without extensive removal of bone substance, considerable shortening of limbs and pseudarthroses are frequently avoided. Sinuses are scarcely ever seen in cases thus treated. The recovery is all the more complete, for a case of compound fracture of the tibia, the femur or the humerus, sutured after the lapse of a few days only, presents neither the muscular atrophy, the retraction of tendons, nor the joint-stiffness, which, after long periods of suppuration, reduce limbs to the verge of impotence.

Sterilisation of wounds is equally successful in securing, more readily than by the other methods, healing of deep wounds of the soft parts. In reality, since tendons and muscles can be sutured as soon as the wound is sterile, the unions are stronger. Nerve suture likewise is done under excellent conditions. In wounds of muscle, the deep and painful cicatrices, which so hamper the usefulness of a limb, are not produced. It is quite certain that the economies in the amount of pensions paid by the State, obtained by means of the sterilisation of wounds, are very considerable.

\section{Failures and their Causes}

Failures teach more than successes. Therefore it is important to examine in what cases the method fails to sterilise wounds, and what are the causes of these failures.

A. Wounds of the Soft Parts not accompanied by Bone Injuries.-If the surgical sterilisation of a wound be considered as the object of the method, it might be 
asserted that no failure has been observed since the month of December, 19I 5. But if the role of the method is to prepare for the secondary closure of wounds, the proportion of failures rises to about five per cent.

These failures are due to the following causes:-

(a) Errors in the bacteriological examination. In spite of the absence of microbes from the smears, suture of the wound was followed by infection. This accident was very rare and always without serious results. It was met with twice in the course of 333 cases of woundclosing. Wounds, the seat of infection, were reopened and sterilised in a few days. This accident was the consequence of specimens for the smears being badly taken; and can be avoided by taking multiple specimens, especially from the most obscure parts of the irregularities of the wound.

(b) Loss of tissue-substance. In some cases, the loss of integumental substance was very extensive, and union impracticable. In other cases, union became possible, if traction more or less great by sutures were employed. But these cut the skin and union remained imperfect.

(c) Closure without bacteriological examination. It sometimes happened that, seeing a wound of good red colour, without secretion, and with margins perfectly supple, the surgeon did not wait for a laboratory report, and sutured. Under these conditions, the operation sometimes resulted in failure. This mistake has not been committed in our hospital since the month of December, 1915. Before that date, it happened several times.

When the treatment was commenced after a period of suppuration more or less long, numerous failures of 


\section{TREATMENT OF INFECTED WOUNDS}

the method might have been expected. Nevertheless, all the suppurating wounds arrived at the stage of surgical sterilisation, and no failure was registered. The closure of these wounds was practised at a later date than when dealing with fresh wounds, but nearly all the cases were sutured.

B. Wounds of the Soft Parts accompanied by Injuries to Bone.-In compound fractures, and especially in those which had suppurated before the commencement of sterilisation, we did not always achieve surgical sterilisation. From this point of view, the results of treatment of compound fractures can be clearly separated from those of wounds of the soft parts. Failures were more frequent. We look upon as failures cases in which some microbes persisted in the secretions up to the spontaneous closure of the seat of fracture, and those in which a sinus or fistula persisted.

We have observed no failure in the treatment of compound fractures of the small bones, short bones, radius, and ulna. But some compound fractures of the humerus, tibia, and femur did not respond completely to treatment. The statistics of these cases will not afford any indication of interest, because the methods have been progressively modified, and the results are improving more and more. In the last fifteen cases of fracture of the humerus which have been under our care, several of which were freely suppurating at the commencement of treatment, only four were not sutured. In two cases, suture was not practised because of loss of substance. In only two cases surgical sterilisation of the seat of fracture was not attained. One case was a "smashup" of the end of the diaphysis and the adjoining 
head of the humerus, and the other case was a highly comminuted fracture of the shaft. In both cases the secretions contained some microbes up to complete cicatrisation. Recovery took place without a sinus remaining.

In compound fractures of the tibia, the loss of substance is often too great to allow of the soft tissues being completely brought together. In similar fractures of the femur, approximation of the tissues was always possible, but microbes often remained in the secretions, and prevented suture being carried out.

\section{Practical Value of the Method}

The results observed at Compiègne showed us that suppuration of wounds can be suppressed, and that the majority of wounds are capable of being sterilised and sutured. The practical value of the method depends upon the possibility of its being employed at other hospitals. As a matter of fact, the objection has been raised that the chemical sterilisation of wounds is, technically speaking, too delicate a method for general employment. It will be as well, therefore, to demonstrate how, without increase of staff, by the aid of apparatus whose cost does not exceed a dozen francs per bed, using substances which cost much less than ether, hydrogen peroxide, or alcohol, usually employed in treatment of wounds, it has been possible to apply the abortive treatment of infection and the curative treatment of suppuration in some of the hospitals at the front (ambulances de l'avant), and in some of the territorial hospitals. 
A. Abortive Treatment of Infection.-The abortive treatment of infection, instituted at Compiègne in the spring of 19I5, was tried experimentally in the hospitals from the month of July in the same year by le Médecin Principal Uffoltz, Directeur du Service de Santé d'un Corps d'Armée. From that date M. Uffoltz and his colleagues demonstrated that under the ordinary conditions of a field-hospital, the method could be employed almost in its entirety, and that a considerable improvement in results was the consequence. In one of the hospitals under the charge of M. Uffoltz, le Médecin-Major Perret succeeded in banishing wound infection almost completely. The ordinary staff was able to apply the method in accurate detail. The demonstration of the practical value of the method in the field-hospitals was brilliantly achieved by MM. Hornus and Perrin, who succeeded in protecting their cases from septic accidents, in preserving limbs with enormous injuries, and in cutting short to a large extent the duration of treatment, by the secondary union of wounds.

Nor is the number of cases any obstacle to the employment of the method. At the hospital at La Panne, which contains from 600 to 700 wounded, M. Depage and his colleagues proved that the sterilisation of wounds could be carried out on a large scale. It had, however, been said that the small size of our hospital at Compiègne allowed us to lavish an amount of attention on our cases which would have been impossible if these cases had amounted to several hundreds. Therefore it is important to realise that the staff of a great hospital succeeded in practising the sterilisation of wounds in every case, in following the progress of chemical cleansing upon the 
bacteriological charts, and in closing wounds as soon as they ceased to harbour microbes. In this hospital, which contains nearly 700 wounded, suppuration was almost completely abolished, without the necessity of increasing the personnel or altering the general organisation.

The results observed in M. Uffoltz' field-hospitals and M. Depage's hospital show that the abortive treatment of infection can be realised in the "formations sanitaires" at the front, when these are well organised and controlled.

Where wounded men are arriving in large numbers, and it is necessary to evacuate them rapidly on the hospitals in the rear, the abortive treatment of infection may still be attempted. As the surgical intervention which precedes chemical sterilisation is identical with that which should always be practised, it matters little that the treatment may be interrupted a few hours or days after its institution. In this case, indeed, the wounded man is in the same position as would be his after the ordinary treatment; but he has benefited by the commencement of the sterilising process. Moreover, the treatment can be resumed the moment he reaches the hospital in which he is finally to remain.

B. The Disinfection of Suppurating Wounds.-The application of the method, which is difficult and occasionally impossible in field-hospitals crowded with wounded men, can always be carried out in the territorial hospitals, in which men with suppurating wounds are received after the lapse of a few days or weeks. The observations made at the Compiegne hospital since September, 1916, have shown that wounds already deeply infected, whether accompanied by fractures or 
otherwise, cán be sterilised as well as fresh wounds, although somewhat more slowly. When the patient had gone through the commencement of chemical sterilisation by Dakin's solution in the advanced field-hospital (firstline ambulance), the wound was comparatively lightly infected, and the rate of disinfection was more rapid.

Identical results have been obtained in all those hospitals in which the method has been applied in all its details. Thus in M. Tuffier's hospital at Saint-Germain, and in M. Chutro's wards at the Buffon hospital, suppurating wounds became a thing of the past. Suppuration in wounds, in short, ought not to exist, except in exceptional cases and for a limited time. The presence of pus in a hospital denotes that the technical methods employed there are defective. Every wounded man whose injury suppurates has a right to call his surgeon to account.

\section{Conclusions}

Since our methods have been employed with success under the ordinary conditions of field and base hospitals, the sterilisation of both fresh and suppurating wounds ought to be practised almost everywhere. But surgeons should not forget that all the details of the method have been studied experimentally and established in a certain way to produce a certain result. Neither the preparation of Dakin's solution may be modified, nor the processes for the mechanical and chemical cleansing of wounds. It is indispensable to learn the method before attempting to apply it, and this apprenticeship demands several weeks, even from an experienced 
surgeon. But we can be quite sure that, applied in their entirety, the methods just described will produce the desired results. Admitted, their use exacts more precision and more care than the old methods, for any approach towards technical perfection requires more elaborate apparatus and a more specialised staff. But efforts of no great magnitude on the part of doctors and nurses will most certainly yield an immense improvement in results.

The nation has the right to ask from the medical corps that progress in the treatment of the wounded which is so acutely needed. 


\section{APPENDIX}

\section{CHLORAMINE PASTE}

THE formula for chloramine paste is not given in the French edition. M. Carrel informs me it is made as follows :-

$$
\begin{aligned}
& \text { "Chloramine T . . . 10 } \\
& \text { Stearate of Soda . . . } 70 \\
& \text { Water . . . . . } 1000
\end{aligned}
$$

The preparation of this substance is somewhat difficult, and it should be made by means of a mechanical mixer, in order to obtain a thoroughly homogeneous paste."

HERBERT CHILD. 


\section{INDEX}

ABORTIVE treatment of infection, 254,255

Abscess, gas-producing, 125

- lymphangitic, 125

Aërobic microbes, 74

Alkali, free, in ordinary hypochlorite, 22

A mputations, almost always necessitated by infection, I, 2

_ in gas gangrene, 124

-, reduction of number by treat. ment, 244-6

Anaesthesia in preparation of wounds, 113,114

Antisepsis, conditions of, 40, 4I

Antiseptics, action of, 64,65

-, concentration of, to be maintained, 84, 85

- duration of application, 8690

-

_- germicidal potency of various, 18-29'

Leishman, Wright, etc., 4

-, method of applying, 8I-84

-, qualities reqnired in, 5,6

- , selection of, 15,16

cation of, I4
Antiseptics. See Dakin's Solu'SION; HYPOCHLORITE, etc. Asepsis, a condition of wound closure, 205

- apparent, of fresh wounds, 73,74

_- bacteriological, often obtained

by chemiotherapy, 203, 204

- , substitute for antisepsis, a,

5

_-, surgical, indications of, 18I

BACTERIA of war-wounds, 6

- destruction of, by antiseptics, 64,65

-__, development of various species at various stages, $73-77$

_-, multiplication of, rapid, 75

- See Microbes

Bacteriological examination, of wounds and secretions, importance of, 15

- danger of wound closure without, 251

- smears, method of, 42,43

-, technique of, 181-204

Beck's paste, 213

Berthollet discovers hypochlorite of soda, 93

Blood.clots, removal of, 80 
Blood-serum hinders bactericidal action of antiseptics, $16-18$

Bone, grafting of, 134 - loss of, made good, 80,213

Bony cavities, filling of, 80, 213 - splinters, resection of, 131

Boric acid, in Dakin's Solution, 22, 23, 94, 95

Brain, wounds of the, preparation of, 128,129

-, special method of instillation practised in, 154,155

Capillary action, failure of, as a means of applying antiseptics, 82

Carbolic acid, Lister's use of, 3

- low bactericidal potency of, 18

Carrel, A., investigates chemiotherapy, 6

Carrel method, the, opposed by French medical service,

II, 12

plorable results of, 13

- technical principles of the, 14-92

Chemical sterilisation of wounds. See Abortive Treatment; Chemiotherapy ; SterilisaTION, etc.

Chemiotherapy, principles of, 14

- of general infections, 39

- of local infections, 39,40

Chloramine paste, 258

\section{- T, 70}

Chloramines, interaction with tissues, 71,72

$67-71$

Chutro, Señor, his work in Paris, Io
Cicatrices, enclosure of microbes in, 78

Cicatrisation, conditions and rate of, $4 \mathrm{I}$

delayed by nicrobial activity, 41,42

- , formula and curve representing the laws of, 44-47, I33, 134

Cleansing of wounds. See PREPARATION

Clinical examination of infected wounds, I12, I13, 173-180

Closure of wounds, after suppuration, 215

-, after the twelfth day, 215

- , before the twelfth day, 214, 215

-

- dangers of immediate, 119

- primary closure, 206, 207

- , secondary closure, 207, 208

_- technique of, 209-213

Complications, diminution of, 243 , 244

Compresses, harmful effects of, 77 , 80

Conclusions, 236, 237

Contact, importance of perfect, between antiseptic and microbes, 79

- importance of prolonged, 87 , 88

Cost of treatment, decreased by the Carrel method, 249

DAKıN, H. O., his investigations of chloramines and hypochlorite, 7,8

- - - antiseptics, properties of various, 19-2I

- - - blood-serum, deterrent action of, $16-18$ 
Dakin, H. O., his investigations of chemiotherapy, 6

- prepares hypochlorite. See HYPOCHLORITE OF SODA; DAKIN's SOLUTION

Dakin's solution, bactericidal potency, of, in vitro, 27, 28 ; in vivo, 29, 30

- compared with Javel's and Labarraque's solutions, 105, 106

- compared with other antiseptics, 32-34

- concentration, proper degree of, 86,108

$\longrightarrow$, concentration decreased by contact with tissues, 85

- cost of, 249

-, Daufresne's method of preparing, 95-100

$\longrightarrow$, instillation of, 162-168

-

- perfected method of preparation, 95-98

-, preparation of, 94

$\longrightarrow$, technique of manufacture, 93108

Daufresne on the bactericidal potency of Dakin's solution, 27-29

- his method of preparing $\mathrm{Da}-$ kin's solution, 95-100

_ investigates deterrent action of blood-serum, 16-18

Deep wounds, difficulty of sterilising, 79

Depage, Dr., introduces the Carrel method at La Panne, Io

Dimensions of wound and time of sterilisation, relation between, 89

Distributor tubes (glass) for instillation, 188, 189
Drainage of wounds, II 8 , I 19

Dressing of irrigated wounds, 157I6I

Drop-counter for instillation apparatus, 143

Duration of treatment decreased by early anatomical repairs, 247,248

- by secondary closure, 246 , 247

EAU DE JAVEL, compared with Dakin's solution, 105, 106

- action of, on tissues, 23, 24

$\longrightarrow$, dangers of, $21,93,107$

- use of, in the present war, 25, 62

Elbow, injuries to the, 23I

Electro-vibrator, Bergonié's, II 7

Errors in bacteriological examination, 251

- of technique, 168-172

- of treatment, causes of, 106108

FAILURE, causes of, 250-253

Femur, fractures of the, 226-230, 236, 238-242

Flavine, retards repair of tissues, 58-60

Forearm, fractures of the, 235,236 Foreign bodies, search for, I1I, I16-1 18, 128

Fractures, closure of, 212, 213

$\longrightarrow$, compound, and joint injuries, I 19,120

- infected, treatment of, 126, 127, 130-1 32

- , sterilisation of, 133, 221,242

-

-, surgical cleansing of, $130-132$

Fresh wounds, sterilisation of, 217 , 218

$$
17^{*}
$$


GANGRENOUS infection, IIO

wounds, sterilisation of, 218 . 220

Gas, given off by anaesthetic culture, 74

Gas gangrene, I

- causes of, 80

$\longrightarrow$ treatment, IIO

-, types of, 1 22-125

Gauze, improper use of, $8 \mathbf{1}, 82$

Grafting, of bone, 134 of adipose tissue, 213

- osteo-periosteal, 248, 249

Guillot and Woimant, researches of, at Compiègne, I I

HAEMORRHAGE, secondary, 127, 128

Haemostasis of wound surfaces, importance of, 80, 116, 127, 128

Hydrogen peroxide, low bactericidal potency of, in vivo, 19

Hypertonic saline solutions, uselessness of, 32, 33

Hypochlorite, electrolytic, 24

- may be rendered stable, 24

Hypochlorite of magnesium, advantages and defects of, 26

Hypochlorite of soda, Dakin's, 7,8

,- - properties of, 2 I-27

- , action of, not due to alkalinity, 35

- , on anatomical elements, 39

,-- , on cicatrisation of infected wounds, 47-52

,-- , on that of aseptic wounds, 52-61

,-- , on tissues provided with circulation, $41-42$

$\longrightarrow$, , on toxins, 35-37

$\longrightarrow$, commercial, 20
Hypochlorite of soda, dangerous if injected into circulation, being strongly haemolytic, 37 - action of, mode of, 64-7I

,-- , on tissues, slight, 23,24

- destroyed by the tissues, $61-64$

- interaction of, with dead and living tissues, 38-4I

- renewal of, frequent necessity of, 84-86

- toxicity of, low, 37

- See Dakin's Solution

Humerus, fractures of the, 222, 223 , 230, 231, 233

IMMOBILISATION of wounded limbs, 162

Incapacity, reduced by abortive treatment, 249, 250

Incision of wounds, $8 \mathrm{I}$

Infected wounds, impotence of old methods of treatment of, I See Preparation; Clinical AND BACTERIOLOGICAL EXaMINATION ; STERILISATION, etc. Infection in wounds, advantages of suppression of, 2

—, abolition of, now possible, 8

—, gas-producing, $122-125$

-, local character of initial stages, 5

- - topography of, studied by means of smears, 180-204 $\longrightarrow$, , 73-80

Inflammatory stage of wound infection, 110

- danger of intervention during,

121, 122

Instillation of antiseptics, 85,86

- continuous, intermittent, automatic, etc., 162-167

- duration of, 167,168

_ 
Instillation, technique of, 135-172

Instillation tubes, $135^{-1} 39$

-

\section{5}

- , arrangement of, in wounds, $146-156$

_- fixation of, to dressings, 160 , I6r

JoINT injuries, cleansing of, 120, I2I

-, sterilisation of, 230-234

-, suppurating, 127

KNEE-JOINT, injuries to the, 231234

LABARRAQUE'S solution, action of, on tissues, 23,24

- compared with Dakin's solution, 106

- dangers of using, in place of latter, 2 I, 107

La Panne, results obtained by

Carrel method at, 254, 255

Leucocytes, dissolution of, 39, 65

- importance of destroying on surface of wounds, 40

- in wounds undergoing sterilisation, 198, 199

Lime, chloride of, titration of, 95-97 Lister, value of his work belittled by later surgeons, 3, 4

Living tissue, resistance of, to hypochlorite, 66

Lumière, M., on action of hypochlorite on microbial toxins, 3537

MAGNeSIUM chloride, inefficacy of, 33,34

- retards cicatrisation, $5 \dot{6}, 5^{8}$

Magnesium hypochlorite, 26
Mahu, M., treats mastoiditis with Dakin's solution, II

Mechanical action of hypochlorite solution nil, 32

- cleansing of wounds, $80,8 \mathrm{r}$

Medullary plugs, removal or drainage of, 131

Microbes, counted by means of smears, 185

- encapsulation of, 78,133 , 134

- found on surface of wounds, 175,176

-, multiplication and destruction of, 188,189

—, persistence of, due to foreign bodies, 197, 198

$\longrightarrow$, scarcity of (apparent), in fresb wounds, 187

$\longrightarrow$, varieties of, 193

MONONUCLEAR cells in wound secretions, 198

Mortality, great reduction of, by Carrel method, 243

Mösetig's paste, 213

NECROSED tissue, action of Dakin's solution on, 38

- protects microbes, 77

$\longrightarrow$, removal of, 80

Nouy's formula for determining rate of cicatrisation, 45

OInTMEnTs, defects of, 27

Oleate of soda, 134

Osseous fissures render sterilisation difficult, 79

Osteo-myelitis, 2, 79

PARA - TOLUENE-SODIUM - SUlPhoCHLORAMINE, 70

Patella, fracture of the, 222 
Phagocytes on wound-surfaces, importance of destroying, 39

Phagocytosis in walls of wounds, 39

Phlegmonous infections, I10, 125, 126

- , sterilisation of, 218-220

Physiological saline solution, inefficacy of, 32, 33

Polynuclear cells in wounds under treatment, 39, 75, 198, 199

Powdered antiseptics, 27

Pozzi, M., demonstrates value of chemiotherapy, 8

Pre-inflammatory stages of wounds, 809

- , the best time for intervention, I I I

Preparation of wounds for sterilisation, 80, 8r

-

-, chemical cleansing, 129, 130

_-, compound fractures, cleansing of, II9-12I

-, drainage, I 8 , I 19

—, foreign bodies, search for, I $16-118$

- , haemostasis of walls, I 16

_, mechanical cleansing, Io9-

\section{I I}

I $14-116$

-

$\longrightarrow$, technique of, I I I-129

Preventive treatment, advantages of, 7

QUÉnu, on results of the Carrel method, 9

RADIOLOGICAL examination of wounds, I12, I1 3

Reinfection, from cicatricial tissues, $78,133,134$
Reinfection from sinuses, 190-193 Repair, action of hypochlorite on, $47-6 r$

- , rate of, $44-47$

Repairs, anatomical, influence on duration of treatment, 247-249

Research, failures and errors in, 2, 3 Results of Carrel method, 216, 217 Rubber tubes, in application of antiseptic, 82-84

SCARS, results of, 2

Secondary or operative infection, 76 Secretion of wounds, the bacteriological study of, I 5, I8I-204

Septicaemia, danger of, III

Serums, useless in cases of general wound infection, 6

Sherman, Dr., on the Carrel method in industrial accident cases, Io Shock, treatment for, III Sinus, failure due to, 252 Soda, action of, on tissues, 65 Soft parts, wounds of the, 216 Smears, asepsis indicated by, 202, 203

- , secretions of wounds studied by means of, 18I-204

-, technique of, 182-187

- , topography of infection examined by means of, 73 , 74

_- uselessness of, while haemorrhage persists, 186

- value of the method, 200

Splinters, bony, excision of, 120 Statistics of results obtained, 9, I0, 252-255

Sterilisation of wounds, chemical, possibility of, denied by Wright, 4,5

- 
Sterilisation of wounds, prolonged contact between antiseptic and the tissues essential, 87,88

- , results obtained, 216-242

- - spontaneous occurrence of, impossible, 29, 30

-, technique of, $135^{-1} 72$

- time required for, in relation to size of wound, 89

Strapping, closure by, 209

Stumps, covered by traction, 2 I I

Suppurating wounds, closure after sterilisation, 252

- - disinfection and abolition of, 253,256

-

-

Suppuration, abolition of possible, 8 - can and should be abolished,

$253,255,256$

-

Surgical cleansing of wounds, 8o,

81. See Preparation

Surgical intervention, dangers of, in highly infected wounds, 125

Suturing of wounds, 76,77

- of muscles, nerves, etc., 212

- peril of premature, 206, 207

-, technique of, 212

208

Thigh, fractures of the. See Femur Tibia, fractures of the, 223-226, 239, 240

- , causes of occasional failure in treating, 253

Tissues, the, action of Dakin's solution on, 37-39

- - action of hypochlorite on, 66

- action of, on hypochlorite, 4 II -44
Tissues, importance of not injuring, 5,6

Titration of chloride of lime, 95

- of Dakin's solution, 99-102

Traction, closure by, 210-2 12

Tubes, instillation, 135-162. See INSTILLATION

Tuffier, M., his treatment of pleurisy, II

on the Carrel method, 9

VINCENT's powdered antiseptics, 27

WASHING wounds, futility of, 86,87

Wounds, bacteriological examination of, 90-92, 181-204

- , clinical examination of, 173180

- closure of, 205-215

--, conditions of those treated, 42,43

- dressing of, $157-160$

_- fractures and wounds of joints, 22I-242

- infection of, 5

- measurement and repair of, 43-45

-

different stages, 73-77

- , preparation of, $109-133$

- - - for treatment, $80,8 \mathrm{r}$

- , septic character of, I

parts, 216, 217

- - of fresh wounds, $21 \%$, 218

- , - of phlegmonous and gangrenous wounds, 118-220

,--1 of suppurating wounds,

221

_- sterilising, method of, $82-84$

-

- - treatment of different types of, 146-157 
Baillide, Tindall \&- Cox, 8, Henrietta Street, Covent Garden, W.C. 2. 





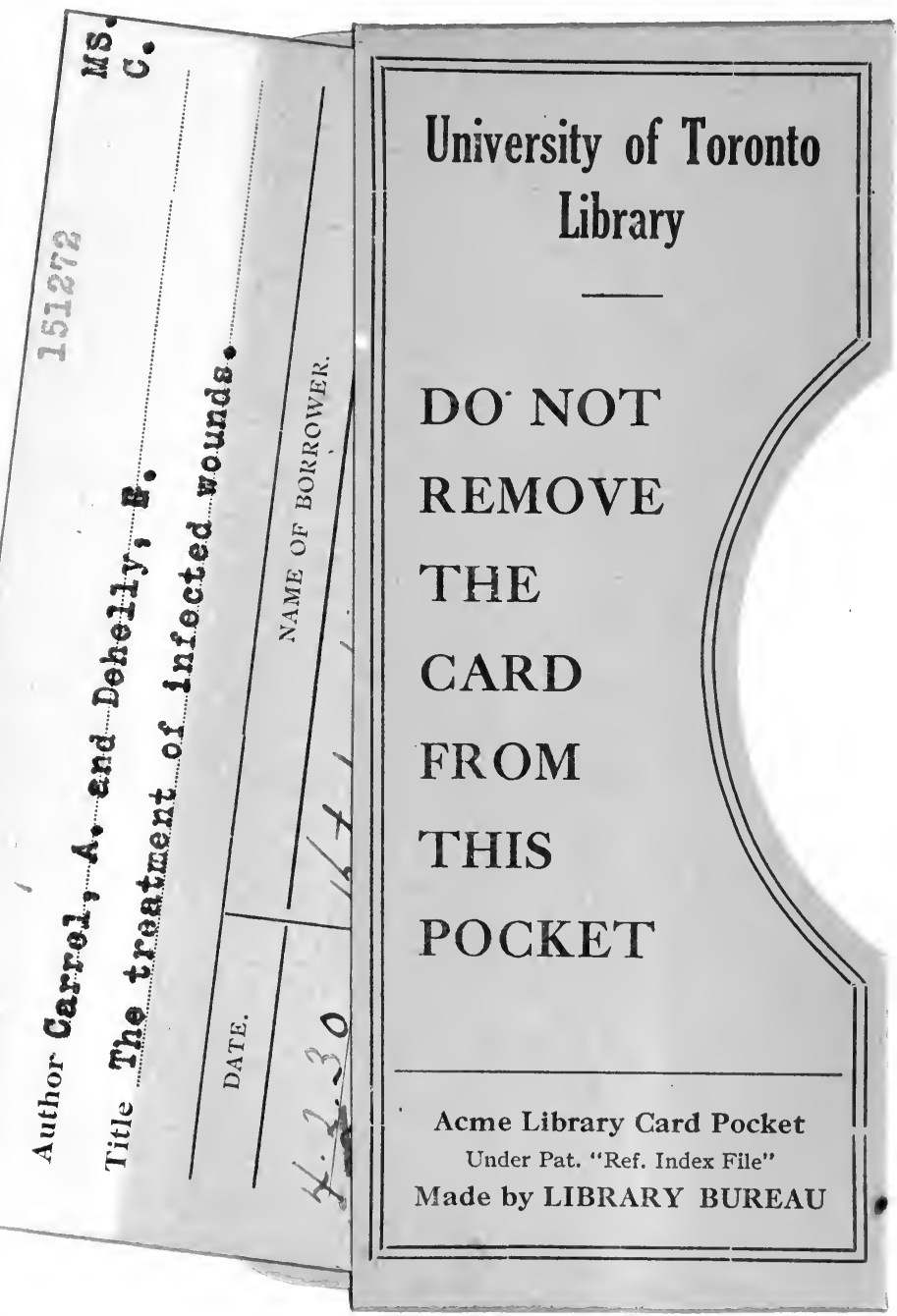


